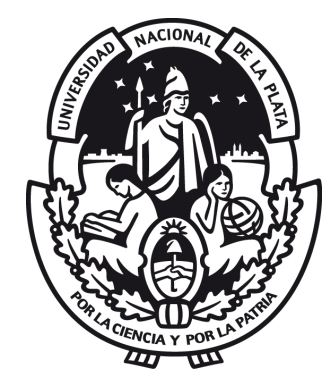

UNIVERSIDAD NACIONAL DE LA PLATA

FACULTAD DE CIENCIAS EXACTAS

DEPARTAMENTO DE FÍSICA

Trabajo de Tesis Doctoral:

\title{
CARACTERIZACIÓN DE PROCESOS DINÁMICOS SOCIALES EN REDES BIDIMENSIONALES Y REDES COMPLEJAS MULTICAPAS
}

Tesista: Lic. Fátima Velásquez Rojas.

Director: Dr. Federico Vázquez.

2018 

A la interdisciplinariedad, por definirme! 



\section{Resumen}

El estudio de procesos dinámicos en sistemas con topologías complejas es un tema de gran interés actual, debido a sus aplicaciones en diversos campos de la ciencia. En particular, los procesos de contacto que surgen de la interacción entre las unidades de un sistema han sido utilizados con gran éxito para modelar diversos fenómenos en el campo de la física. Nosotros nos centraremos en estos procesos aplicados a fenómenos sociales como la formación de opiniones y la propagación de enfermedades en redes bidimensionales y redes complejas múltiples, a partir de simulaciones computacionales y algunas descripciones teóricas.

En el primer capítulo se describen los modelos de partículas interactuantes en la Física Social que constituyen las dinámicas fundamentales utilizadas en este trabajo.

En el segundo capítulo estudiamos un modelo de dinámica de opiniones que explora la competencia entre la persuasión y el compromiso en una población de individuos que interactúan en una red cuadrada bidimensional. Esta competencia es modulada por una variable $r$ para la cual el sistema tiene comportamientos bien diferenciados: centralización y bipolarización.

En el tercer capítulo analizamos la interacción entre dos procesos diferentes, la formación de opiniones y la propagación de una enfermedad en una población de individuos utilizando una red compleja de múltiples capas, donde estas se acoplan a través de sus enlaces. Ambas dinámicas se ven modificadas por la presencia del acoplamiento, cambiando el tipo de transición de fase sana-endémica y la naturaleza del consenso.

En el cuarto capítulo describimos otro modelo sobre redes múltiples: la diseminación de una epidemia y la divulgación de la información sobre el conocimiento de esa enfermedad y sus métodos de prevención. Encontramos que la velocidad de propagación de la información no siempre reduce el brote de la epidemia.

Por último presentamos las conclusiones generales y las perspectivas de este trabajo. 



\section{Agradecimientos}

En momentos como este nos detenemos a mirar atrás para contemplar todo lo que hemos recorrido, pero aún más importante es mirar a nuestro lado para observar quienes nos siguen acompañando en este camino, vaya para todos ustedes mi más sincero agradecimiento.

Al CONICET, por ser el principal sustento económico para llevar a cabo este trabajo.

Al Dr. Federico Vázquez, por plantearme este importante desafío y acompañarme a superarlo con dedicación y paciencia.

A Jesús Fajardo, mi compañero de vida, mi apoyo incondicional diario y la prueba de que con amor todo se supera.

A toda mi familia, en especial a mis abuelos Demóstenes y Zoraida, y a mis padres Rafael y Mercedes, por las llamadas y mensajes constantes, por sus palabras de aliento, por ser mi sostén emocional y por estar siempre presentes aún con la enorme distancia física que nos separa.

A la UNLP, por proporcionar el espacio académico para formarme como Doctora y seguir formándome como Docente.

A las familias Irastorza-Costa y Carlevaro-Orzuza, por su solidaridad, por su cariño sincero y por todo el apoyo brindado desde el primer día.

A todos los compañeros del IFLYSIB, por la buena onda, por hacer de este el mejor lugar para trabajar y compartir. A mis compañeros de oficina, los de ayer y los de hoy, por incorporar cada modismo venezolano y por ayudar en todo momento a mantener el sentido del humor en nuestro espacio.

A los Jurados, por tomarse el tiempo de leer, corregir, y brindar sus valiosas sugerencias.

A todos mis compañeros del CULP, quienes se convirtieron en mi familia platense, en donde en cada ensayo vuelvo a mi hogar.

A todos ustedes INFINITAS GRACIAS!!! 



\section{Índice General}

Resumen $v$

\begin{tabular}{lll}
\hline Agradecimientos vii & vii
\end{tabular}

Índice General $\quad$ ix

$\begin{array}{ll}\text { Introducción } & 1\end{array}$

1. Modelos de partículas interactuantes en la Física Social 5

1.1. Redes Cuadradas Bidimensionales . . . . . . . . . . . . 5

1.2. Redes Complejas . . . . . . . . . . . . . . . . . . 6

1.2.1. Red aleatoria ER . . . . . . . . . . . 8

1.2.2. Redes de Múltiples Capas . . . . . . . . . . 8

1.3. Modelos de Formación de Opiniones $\ldots \ldots \ldots$. . . . . . . 11

1.3.1. Modelo de Ising . . . . . . . . . . . . . . . . 12

1.3.2. Modelo del Votante . . . . . . . . . . . . . . 17

1.3.3. Modelo M: Persuasión y Compromiso . . . . . . . . . 22

1.4. Modelos de Propagación de Epidemias . . . . . . . . . . . . 28

1.4.1. Proceso de Contacto . . . . . . . . . . . . . . 29 
2. La formación de opiniones en redes cuadradas 35

2.1. El modelo M en una red cuadrada . . . . . . . . . . . . . . . . . 38

2.2. Dinámica de coarsening . . . . . . . . . . . . . . . . . . . . . 40

2.3. Tiempos de consenso . . . . . . . . . . . . . . . . . . 45

2.4. Dinámica de las bandas hacia el consenso . . . . . . . . . . . . . 49

2.4.1. $\quad$ Estimación de $\tau_{b}$ considerando dos partículas difusivas. 52

2.4.2. $\quad$ Estimación de $\tau_{b}$ considerando dos barras difusivas. . . 55

2.5. Discusiones finales . . . . . . . . . . . . . . . . . . . . 59

3. Propagación de Opiniones y Enfermedades en Redes Múltiples $\begin{array}{ll}\text { Interactuantes } & 61\end{array}$

3.1. El Modelo . . . . . . . . . . . . . . . . . . . . . . . . . . . . . . 63

3.2. Resultados Numéricos . . . . . . . . . . . . . . . . . . . . . . 68

3.2.1. $\quad$ Efectos de la formación de opiniones sobre la prevalencia de la enfermedad . . . . . . . . . . . . 69

3.2.2. Efectos de la propagación enfermedades sobre el consenso de opiniones . . . . . . . . . . . . . . . . . 76

3.3. $\quad$ Aproximación analítica . . . . . . . . . . . . . . . . . . . . . 80

3.3.1. Prevalencia de la enfermedad . . . . . . . . . . 83

3.3.2. Tiempos de consenso de opiniones . . . . . . . . . 86

3.4. Discusiones finales . . . . . . . . . . . . . . . . . . . . . . . . . . 89

4. Propagación de Enfermedades e Información en Redes Múltiples 93

4.1. Descripción del Modelo SIS-UAU . . . . . . . . . . . . . . . . . . 94

4.2. $\quad$ Aproximación de campo medio . . . . . . . . . . . . . . . 96

4.2.1. Solución para $\gamma=0, \Gamma=0$ y $\kappa=1.0 \ldots . . . . .997$

4.2.2. $\quad$ Solución para $\gamma=0 \ldots \ldots$. . . . . . . . . 99

4.3. Análisis numérico de las ecuaciones . . . . . . . . . . . . . . . . 102

4.4. Discusiones preliminares . . . . . . . . . . . . . . 106 
\begin{tabular}{ll}
\hline Conclusiones & 107
\end{tabular}

A. Análisis de la distancia media de ruptura de la interfase $\left\langle\Delta x^{b}\right\rangle \quad 111$

B. Aproximación de $\tau_{b}^{\mathrm{III}}$ como una ley de potencia 113

C. Deducción de la ecuación de evolución para $\rho^{+} \quad 115$

D. Deducción de la ecuación de evolución para $\rho^{+-} \quad 123$

E. Deducción de la ecuación de evolución para $\rho_{1} \quad 127$

F. Deducción de la ecuación de evolución para $\rho_{10}$

\begin{tabular}{ll}
\hline Bibliografía & 135
\end{tabular} 



\section{Introducción}

En los últimos años hemos sido testigos de una gran expansión en el estudio de la Física Social [1]. Esta rama de la física interdisciplinar utiliza métodos y conceptos de la Física Estadística y de los Sistemas Complejos para el estudio de fenómenos sociales colectivos. Diversos tipos de interacciones sociales entre individuos han sido explorados con el objetivo de hacer un estudio teórico de algunos fenómenos sociales utilizando diversos mecanismos propuestos en el marco de la dinámica de partículas interactuantes, describiendo el comportamiento colectivo con patrones macroscópicos simples.

Al referirnos al estudio de fenómenos sociales, nos referimos al estudio de los comportamientos que transcurren dentro de una sociedad, que pueden ser realizados por algunos miembros o por su totalidad y que implica, necesariamente, relaciones entre las personas. Estas relaciones conllevan, en términos de la Física Social, procesos de contacto: formación de opiniones, auto-organización, cooperación, propagación de epidemias, transmisión de información, intercambios económicos, coexistencia de lenguas, entre otros [1].

Uno de los fenómenos sociales más estudiados por medio de los procesos de contacto es la formación de opiniones en una sociedad. Los estudios de este fenómeno se basan en la observación de acuerdos o discrepancias acerca de un determinado tema entre personas que mantienen una conversación, y que da origen a un comportamiento colectivo (a nivel grupal).

Varios mecanismos han sido propuestos en la literatura de las ciencias sociales que estudian el comportamiento humano, y que intentan explicar el proceso del consenso de opiniones en un grupo de personas [2,3]. La aparición de consenso global es consecuencia directa de la interacción entre las personas que van modificando su postura al conversar con sus pares, generando acuerdos a nivel local, y que terminan produciendo un acuerdo a nivel grupal. 
Uno de los mecanismos más explorados es el de presión social [2], mediante el cual una persona adopta una opinión en base a las opiniones de las personas del entorno que la rodea. Es decir, antes de tomar una decisión, la persona consulta a sus allegados y luego decide de acuerdo a las respuestas que recibe. El modelo del votante [4 7] es uno de los modelos más estudiados que ponen a prueba este mecanismo. En este modelo, cada persona puede elegir entre dos opciones posibles sobre un asunto determinado: SI o NO. Dos personas que mantienen algún tipo de contacto, ya sea físico o electrónico, están conectadas por una enlace, y todas juntas forman una red compleja de interacciones sociales. Cuando una persona tiene que decidir, simplemente adopta la opinión de uno de sus contactos vecinos elegido al azar. Este proceso de imitación local produce una competencia entre dos opiniones a nivel global y conduce, luego de muchas interacciones, al consenso total de una de las opiniones. Este modelo es análogo al modelo de Ising con spin 1/2 que se utiliza para estudiar el ferromagnetismo, donde las opciones SI/NO corresponden a las dos direcciones posibles de los espines $(\uparrow / \downarrow)$, los cuales tienden a alinearse con sus espines vecinos en una red cuadrada, dando origen a un ordenamiento a nivel global que depende de la temperatura. Otro modelo muy estudiado es el de la mayoría [8, 9], donde una persona alinea su opinión con la de la mayoría de sus vecinos, porque siente la presión de opinar como lo hace la mayor parte de su entorno. Este simple mecanismo también genera un consenso global pero en una escala temporal menor que la del modelo del votante.

Los procesos de contacto también han sido empleados en el campo de la epidemiología, para estudiar la propagación de enfermedades contagiosas 10 12. Muchas de estas enfermedades se transmiten a través del contacto físico o por cercanía entre personas. Esto se modela asumiendo que en la población existen personas sanas que son susceptibles a ser contagiadas por sus vecinos infectados en la red de interacciones. Entre los modelos más empleados figuran el Susceptible-Infectado, en el que las personas contraen la enfermedad y mantienen este estado en forma indefinida, como pasa con el HIV. El modelo Susceptible-Infectado-Susceptible, en el que los infectados vuelven a ser susceptibles al sanarse luego de un tiempo y pueden infectarse nuevamente, como ocurre con la gripe, y el Susceptible-InfectadoRecuperado, en el que las personas no pueden volver a infectarse ni transmitir la enfermedad una vez que se recuperan. En todas estas dinámicas aparece una transición entre una fase sana y una fase endémica (donde gran parte de la población contrae la enfermedad). 
Los procesos de formación de opiniones y propagación de enfermedades que acabamos de describir han sido, hasta el momento, estudiados de forma separada. Es natural en las ciencias físicas lograr un aporte al entendimiento de sistemas que se encuentran aislados, debido a que el reduccionismo facilita la formulación y el análisis de estos problemas. Sin embargo, es sabido que los fenómenos sociales de la vida real no están aislados, sino que dependen y se afectan entre sí. En el caso particular de los procesos de contacto antes mencionados, se espera que la propagación de enfermedades afecte la forma en la que la gente se relaciona, modificando las interacciones y por consiguiente la toma de decisiones y formación de opiniones. Por otro lado, los patrones de contacto entre personas se ven influenciados por la opinión personal, dado que una persona tiende a relacionarse con personas que piensan similar, por una cuestión de selección social. La elección del círculo de contactos frecuentes puede influir en la probabilidad que tiene una persona en contraer una enfermedad. Por lo tanto, existe una coevolución entre las dos dinámicas, debido al acoplamiento entre los procesos de opiniones y enfermedades.

Para modelar procesos que incorporen este acoplamiento de la vida real se han propuesto recientemente las redes de múltiples capas 13 17], donde en cada red o capa se propaga un proceso diferente. El acoplamiento se da a través de los nodos, que actúan como nexo entre las diferentes redes. Es decir, se asume que los individuos que forman el sistema son fijos y que existen varias clases de enlaces, una por cada proceso. Existen trabajos donde se pudieron caracterizar estas estructuras topológicas en sistemas reales [14], y se han determinado una serie de propiedades interesantes debidas al acoplamiento, como la facilitación en la propagación de información [16], y la aceleración de procesos difusivos sobre las dos capas [17].

En esta propuesta de trabajo utilizaremos este enfoque de experimentación para contribuir a su estudio. Pretendemos dilucidar cómo estas dinámicas de formación de opiniones y propagación de enfermedades se ven afectadas en presencia de un acoplamiento mutuo, con miras a explorar otras dinámicas que relacionen diversos fenómenos sociales. 



\section{Capítulo 1}

\section{Modelos de partículas interactuantes en la Física Social}

Como se mencionó en la Introducción de este trabajo, los modelos de partículas interactuantes son utilizados para describir algunos de los procesos que ocurren en la sociedad, donde los componentes básicos no son partículas, sino seres humanos que interactúan con sus pares. Esta interacción entre individuos, hace posible que podamos formular modelos matemáticos y computacionales para realizar una descripción más detallada de los fenómenos que queremos estudiar. Proponer estos modelos implica, por una parte, definir reglas microscópicas realistas y luego inferir la fenomenología macroscópica a partir de la dinámica microscópica propuesta. Las características de estos modelos están pensadas en términos de un pequeño conjunto de variables cuya dinámica está determinada por las interacciones sociales. Las preguntas relevantes que se intentan responder se relacionan con la tendencia de los agentes que interactúan a ser más parecidos, llevando al sistema a un orden.

Dos de los procesos más estudiados en una sociedad por medio de la formulación de modelos de partículas interactuantes son la formación de opiniones y la propagación de enfermedades contagiosas. En este capítulo describiremos los modelos fundamentales de ambas dinámicas así como las diferentes topologías utilizadas en la realización de esta tesis.

\subsection{Redes Cuadradas Bidimensionales}

Las Redes Cuadradas Bidimensionales fueron concebidas como herramientas matemáticas, especialmente en geometría y teoría de grupos para describir problemas de índole diversa. Una red cuadrada bidimensional o retículo se suele representar en el espacio como una celda o malla. 
En el campo de la Física, específicamente en ciencia de los materiales y física del estado sólido, una red es sinónimo del esqueleto de una estructura cristalina, una matriz de puntos regularmente espaciados en tres dimensiones coincidiendo con las posiciones de los átomos o moléculas en un cristal. Más en general, modelos que utilizan este tipo de redes se estudian en Física Estadística, muy frecuentemente mediante técnicas de Física Computacional.

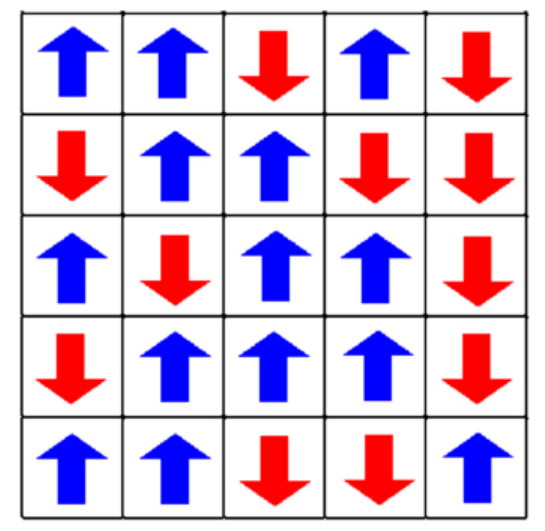

Figura 1.1: Red cuadrada bidimensional con espines en cada casilla cuyas orientaciones son: $\uparrow$ color azul y $\downarrow$ color rojo.

En la Fig. 1.1 se muestra una red cuadrada bidimensional. Cada casilla de la red está ocupada por un espín con cierta orientación, una manera muy común de modelar sistemas magnéticos como el Modelo de Ising [18].

\subsection{Redes Complejas}

Las Redes Complejas son estructuras matemáticas formadas por nodos (o vértices) conectados por enlaces (o aristas) conocidas como grafos (ver Fig.1.2)

El desarrollo en el estudio de las propiedades estructurales y dinámicas de las redes complejas ha sido extenso y ha permitido la publicación de cientos de artículos en revistas de investigación científica de diferentes disciplinas que abarcan física, biología, sociología, economía, por mencionar algunos ejemplos. Este interés en las redes complejas radica en que se ha descubierto que dichas redes abundan en la naturaleza, son parte de nuestra vida diaria y se presentan a diferentes niveles de organización. Por ejemplo, algunas redes biológicas que encontramos en el nivel microscópico son las redes de proteínas, redes neuronales, redes metabólicas. 
Por otro lado, a un nivel de organización mucho mayor, encontramos las redes de comunicación (internet, redes telefónicas, entre otras), redes sociales (amistades, colaboradores científicos, propagación de información, de enfermedades...).

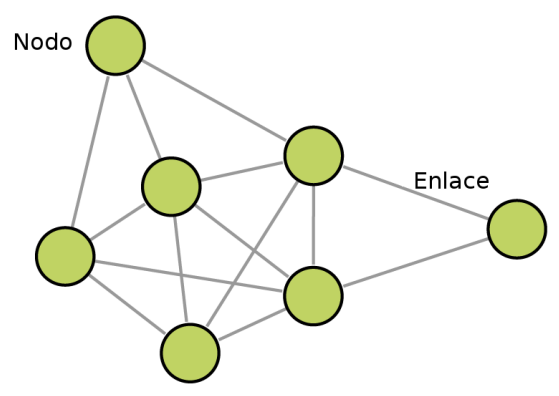

Figura 1.2: Red compleja la cual se compone de nodos y enlaces.

Durante más de un siglo, el modelado de muchos procesos se ha realizado bajo la suposición implícita de que los patrones de interacción entre los elementos del sistema podían ser representados por medio de una estructura regular al estilo de un retículo euclídeo (una red bidimensional en 2D). A finales de 1950, dos matemáticos, Erdös y Rényi (ER), hicieron un gran avance en la teoría matemática clásica de grafos que revolucionaría la forma en que se pueden modelar estos problemas describiendo una red con topología compleja por medio de un grafo aleatorio, estableciendo los fundamentos de la teoría de redes aleatorias.

Con el paso del tiempo y el avance en las herramientas computacionales, surgieron diferentes tipos de redes complejas. En 1998, con el fin de describir la transición de una red regular en una red aleatoria, Watts y Strogatz (WS) introdujeron el concepto de Red de Mundo Pequeño [19], esta red fue utilizada para explicar el experimento realizado hace más de 40 años y que dio lugar al llamado "principio de los seis grados de separación", propuesto por el psicólogo social Milgram a finales de 1960, y que establece que entre cualesquiera dos personas del mundo hay una media de 6 conexiones de amistad, independientemente de lo lejanas que estén dichas personas. Una característica importante y común al modelo de redes aleatorias (ER) y al de mundo pequeño (WS) es que la función de distribución de grados tiene un máximo en el valor medio del grado y decae exponencialmente, lo que quiere decir que casi todos los nodos de la red tienen el mismo número de conexiones. Por ello, es común denominar a estos tipos de redes de manera conjunta como redes exponenciales o redes homogéneas. 
Otro descubrimiento fundamental en el ámbito de las redes complejas, fue la observación hecha por Barabási y Albert (BA) de que muchas son libres de escala [20], es decir, la función de distribución de grados sigue una ley de potencias, que es independiente de la escala de la red. A diferencia de una red exponencial, una red libre de escala es no homogénea: la mayoría de los nodos tienen muy pocas conexiones y hay unos pocos nodos que tienen muchas, de forma que los nodos no se agrupan alrededor de un valor medio característico.

A continuación describiremos los tipos de redes complejas utilizadas en esta tesis doctoral.

\subsubsection{Red aleatoria ER}

Como se mencionó anteriormente, las redes aleatorias fueron estudiadas por Erdös y Rényi (ER) a finales de la década de los 50 [21]. Si consideramos $N$ nodos de una red sin conectar, distribuidos de forma aleatoria, podemos imaginar que en un instante inicial enlazamos dos cualesquiera, de esta forma en pasos sucesivos vamos enlazando aleatoriamente de dos en dos nodos. Los nodos que se encuentren conectados se descartan. Si repetimos el proceso $M$ veces eligiendo un par de nodos en cada paso, al final habremos establecido como máximo $M$ enlaces entre pares de nodos.

Cuando se enlazan los nodos de esta forma aparecen propiedades específicas en la distribución de grado $P(k)$ la cual es una distribución de Poisson:

$$
P(k)=e^{-\mu} \frac{\mu^{k}}{k !} .
$$

donde $k$ es el grado de la red con promedio $\mu=\frac{2 M}{N}$. En la Fig. 1.3 se muestra una red aleatoria ER y su distribución de grado $P(k)$.

\subsubsection{Redes de Múltiples Capas}

La inmensa mayoría de los fenómenos que ocurren a nuestro alrededor, desde los que influyen en nuestras relaciones sociales, hasta aquellos que transforman el entorno general en el que vivimos, incluso aquellos que afectan nuestro propio funcionamiento biológico, son el resultado de la organización a diferentes niveles de una multitud de constituyentes básicos que interactúan entre sí de forma compleja. 


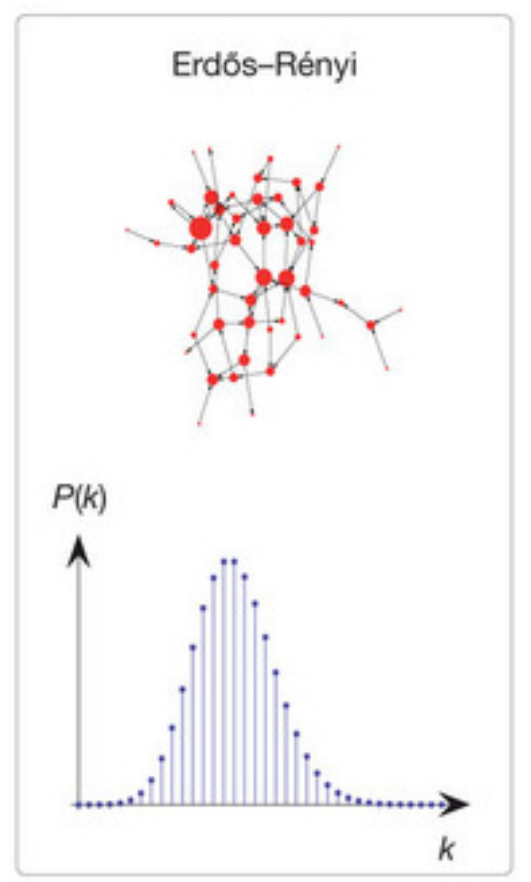

Figura 1.3: Arriba: Red aleatoria ER. Abajo: Distribución de grado $P(k)$ de esta red.

El enfoque tradicional de las Redes Complejas se basa en que cada componente del sistema (o unidad elemental) es representado por un nodo en la red, y cada interacción nodo-nodo es un enlace. Sin embargo, para tomar en cuenta un número más grande de interacciones, tal como ocurre en la vida real, este enfoque nos resulta restrictivo. Para enfrentar esta limitación, recientemente se propuso el formalismo de las Redes Múltiples Capas las cuales representan una herramienta de gran utilidad para comprender la diversidad de interacciones presentes en redes complejas reales $[22]$.

Las Redes Multicapa incorporan explícitamente múltiples canales de conectividad y constituyen el entorno natural para describir sistemas interconectados a través de diferentes categorías de conexiones. Como ejemplos de Redes Multicapas podemos mencionar a las Redes Interconectadas e Interdependientes donde los nodos de cada capa son diferentes, y pueden o no tener enlaces en común entre las capas [22]; otro ejemplo son las Redes Multiplex, las cuales utilizamos en la realización de esta tesis.

Una Red Múltiplex, que en lo sucesivo llamaremos Red Múltiple, contiene un mismo conjunto de nodos y varias capas. Todos los nodos están interconectados, 
esto implica que dos nodos ubicados en dos capas diferentes son en realidad el mismo nodo. En cada capa, los enlaces representan un tipo de interacción entre el mismo conjunto de nodos. La Fig.1.4 muestra un ejemplo de una red múltipe con $m$ capas, las líneas punteadas representan la interconexión en las capas y cada capa está compuesta de $N=6$ nodos.

La conectividad de los nodos puede ser diferente en cada capa de la red, y cada capa caracteriza un tipo de relación entre los agentes.

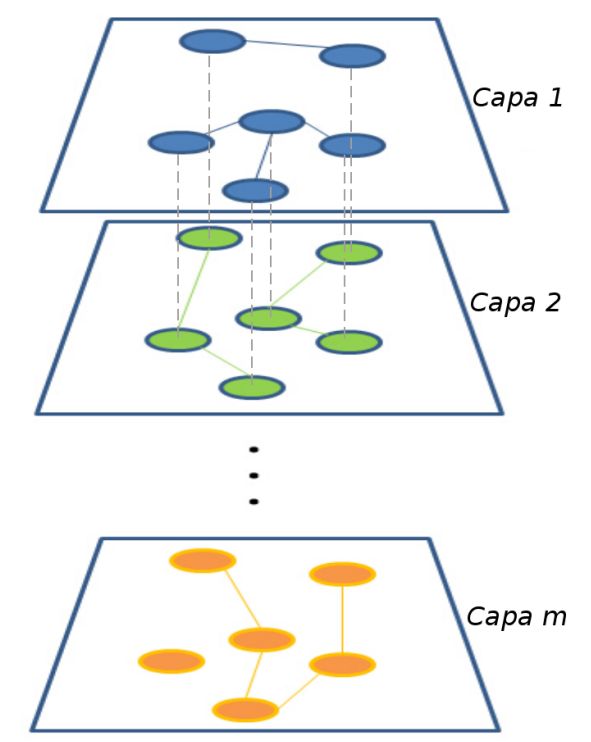

Figura 1.4: Ejemplo de una red múltiple con $m$ capas.

Las relaciones entre miembros de una comunidad que tienen lugar dentro de diferentes grupos (niveles o capas), por ejemplo: una capa de contactos de Facebook, una capa de compañeros de trabajo y una capa de amigos que se reúnen frecuentemente representan una Red Múltiple. Otro ejemplo interesante son las redes de transporte donde cada medio existente representa una capa: Autobús, Subterráneo y/o Tranvía. En este tipo de redes los nodos representan estaciones y los enlaces son las rutas o trayectos entre esas estaciones.

Una vez descritas las topologías a emplear, a continuación, detallaremos los modelos de formación de opiniones y propagación de enfermedades en los cuales se basa esta tesis. 


\subsection{Modelos de Formación de Opiniones}

En los últimos años, los físicos han intentado estudiar los fenómenos sociales a partir de las interacciones entre los individuos. Con frecuencia, durante estas interacciones las personas se enfrentan con un número limitado de posiciones en un tema específico, el cual podría tener un valor mínimo de dos, por ejemplo, a favor de una reforma (opinión +1 ) o en contra (opinión -1). Esto puede mapearse en modelos de espines [1], identificando previamente la opinión de un individuo con el espín de una partícula. Un cambio en la opinión de un individuo después de encontrarse con otro, puede representarse como el cambio de orientación de una partícula en $180^{\circ}$ debido a las interacciones con sus vecinos.

En los modelos de formación de opinión es un tema común el estudio de la transición de un estado inicialmente desordenado a una configuración que muestra orden (al menos parcialmente), lo que se conoce como consenso de opiniones. Este estudio tiene implicaciones y particularidades que iremos viendo en el desarrollo del capítulo.

A continuación describiremos con más detalle algunos modelos de formación de opinión, los cuales constituyen las dinámicas fundamentales de buena parte del trabajo que llevamos a cabo. Además, con el fin de desarrollar una intuición sobre el comportamiento de estos modelos, simulamos las dinámicas y reprodujimos los resultados en topologías variadas.

Empezaremos describiendo el Modelo de Ising. Este modelo no sólo es relevante como un modelo de física, también se puede ver como un modelo muy simple de una dinámica de opinión en el que los agentes están influenciados por el estado de la mayoría de los vecinos con los que interactúan. Modelos de este tipo fueron propuestos por Galam 23, 24] y constituyen los trabajos pioneros del tema.

Luego describiremos el Modelo del Votante [5], uno de los pocos procesos estocásticos del no equilibrio que se puede resolver exactamente en cualquier dimensión [25]. En este modelo, un agente escoge a uno de sus vecinos al azar y copia su opinión.

Finalmente, analizaremos el Modelo M propuesto por La Rocca y colaboradores [26], donde se estudia la competencia entre dos mecanismos para la formación 
de opiniones: la persuasión y el compromiso. De acuerdo a cuál de ellos domine, la opinión de la población muestra características definidas de polarización y centralización.

\subsubsection{Modelo de Ising}

El Modelo Ising es un modelo fundamental de la Física Estadística que describe la dinámica de ordenamiento y transiciones de orden-desorden en sistemas ferromagnéticos [18]. En este modelo, cada sitio $i$ de una red cuadrada está dotado de un espín $\sigma_{i}$ que puede tomar uno de dos estados discretos posibles $\sigma_{i}= \pm 1$, por lo que un sistema con $N$ espines tendrá $2^{N}$ estados disponibles. Además, existe una interacción a primeros vecinos que tiende a alinear sus espines, favoreciendo el orden ferromagnético 25. El hamiltoniano resultante para el sistema es:

$$
\mathcal{H}=-J \sum_{\langle i, j\rangle} \sigma_{i} \sigma_{j}
$$

donde la suma es sobre todos los pares de sitios vecinos $i$ y $j$ denotada por $\langle i, j\rangle$. Por lo tanto, los espines vecinos que apuntan en la misma dirección (paralelos) aportan un factor $-J$ a la energía total, mientras que los pares que apuntan en dirección opuesta (antiparalelos) contribuyen con un factor $+J$.

Entre los posibles tipos de dinámica existentes para este modelo, la más común es la de Metrópolis-Monte Carlo [27], en la que el giro de un espín se acepta con probabilidad $e^{-\Delta E / k_{B} T}$, donde $\Delta E$ es la diferencia de energía implicada en el giro, $T$ la temperatura y $k_{B}$ es la constante de Boltzmann.

Una cantidad importante que caracteriza este modelo es la magnetización $m$ que se define como:

$$
m(T)=\frac{1}{N} \sum_{i}^{N} \sigma_{i}
$$

Es conocido que para dimensiones espaciales $d>1$ el sistema experimenta una transición de fase continua en función de la temperatura [28]. Pasa de una fase desordenada de alta temperatura donde $m=0$ (fase paramagnética) a una fase de baja temperatura donde $m>0$ (fase ferromagnética). En el límite termodinámico $N \rightarrow \infty$, el valor crítico de la temperatura para la cual la magnetización se hace

cero (temperatura crítica) es $T_{c} \approx 2.269185 \frac{J}{k_{B}}$ y determina el punto de transición entre las dos fases. 

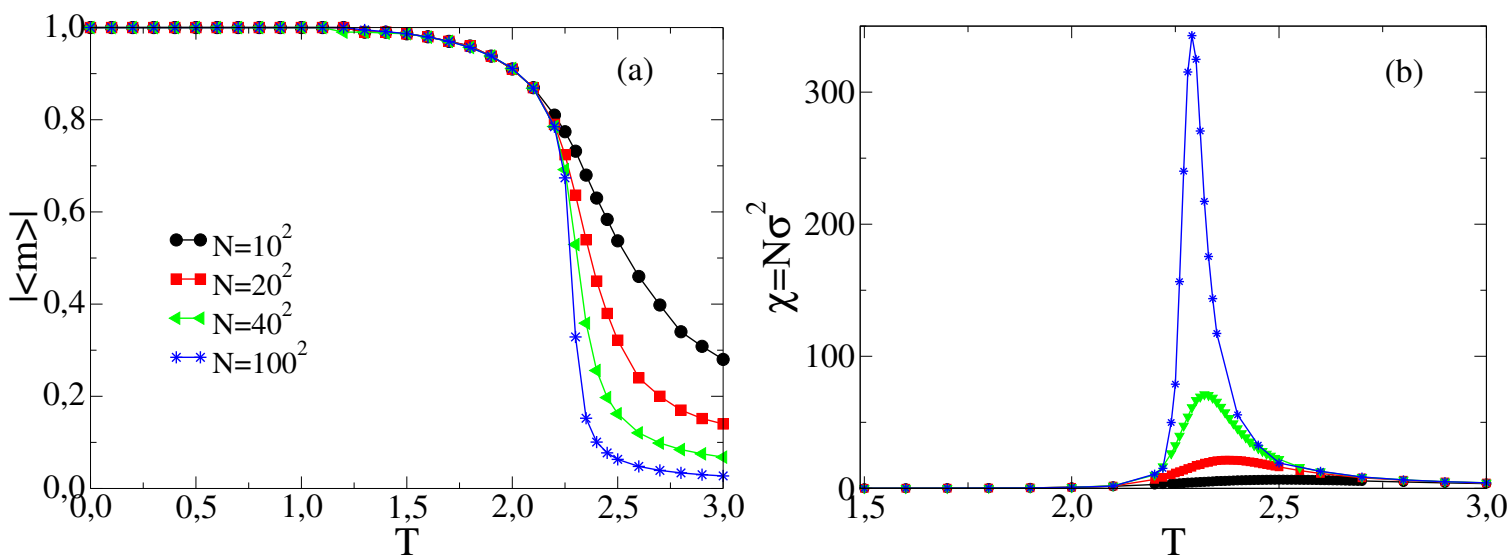

Figura 1.5: Promedio del valor absoluto de la magnetización $|\langle m\rangle|$ vs $T$ en 2D (panel (a)) y Susceptibilidad magnética $\chi$ en función de la temperatura $T$ en 2D (panel (b)) para diferentes tamaños del sistema $N=10^{2}$ (círculos), $N=20^{2}$ (cuadrados), $N=40^{2}$ (triángulos) y $N=100^{2}$ (estrellas). En la transición, la magnetización disminuye rápidamente, mientras que la susceptibilidad magnética alcanza su punto máximo $\left(\chi_{\text {máx }}\right)$ en el valor de la temperatura crítica cuando el sistema se hace más grande.

Reproducimos la dinámica de Metrópolis-Monte Carlo en redes cuadradas de dos dimensiones con condiciones periódicas de contorno. Para determinar la ubicación de la transición de fase en el modelo de Ising bidimensional, corrimos simulaciones de la dinámica del modelo y graficamos la evolución temporal de la magnetización. Una vez que el sistema llegó a un estado estacionario, medimos la magnetización estacionaria en cada realización e hicimos estadística sobre $10^{4}$ realizaciones. Calculamos el valor medio de la magnetización $|\langle m\rangle|$ y la desviación cuadrática media de esta cantidad $\sigma$.

La susceptibilidad magnética es una medida de las fluctuaciones de la magnetización a diferentes temperaturas y es proporcional a la dispersión cuadrática media de la magnetización $\left(\chi=N \sigma^{2}\right)$ con $N=L^{2}$ y $L$ representa la longitud del lado de la red cuadrada. En la Fig. 1.5 se muestran los resultados para redes cuadradas de tamaño $N=10^{2}, 20^{2}, 40^{2}$ y $100^{2}$. En el panel (a) se muestra el valor medio de la magnetización $|\langle m\rangle|$ en función de la temperatura, vemos que en el punto de transición, la magnetización disminuye rápidamente. En el panel (b) se graficó la susceptibilidad magnética $\chi$ vs $T$, la altura de los picos de la susceptibilidad $\left(\chi_{\text {máx }}\right)$ aumenta cerca de la temperatura crítica $T_{c}$ cuando el tamaño del sistema aumenta. 
En el campo de las transiciones de fase, el cumulante de cuarto orden de la magnetización $(m)$ se llama Cumulante de Binder $\left(U_{\langle m\rangle}\right)[29$ y se usa para localizar la transición de fase en un sistema mediante la intersección de las curvas para diferentes valores de $N$. El cumulante de Binder se define como:

$$
U_{\langle m\rangle}=1-\frac{1}{3} \frac{\langle m\rangle^{4}}{\left(\langle m\rangle^{2}\right)^{2}} \text {. }
$$

La Fig. 1.6 muestra el cumulante de Binder para el modelo de Ising bidimensional. El punto de intersección de las curvas corresponde a la temperatura crítica para la que ocurre la transición de fase en nuestras simulaciones $\left(T_{c} \approx 2.269 \frac{\mathrm{J}}{k_{B}}\right) \mathrm{y}$ coincide con el valor teórico conocido.

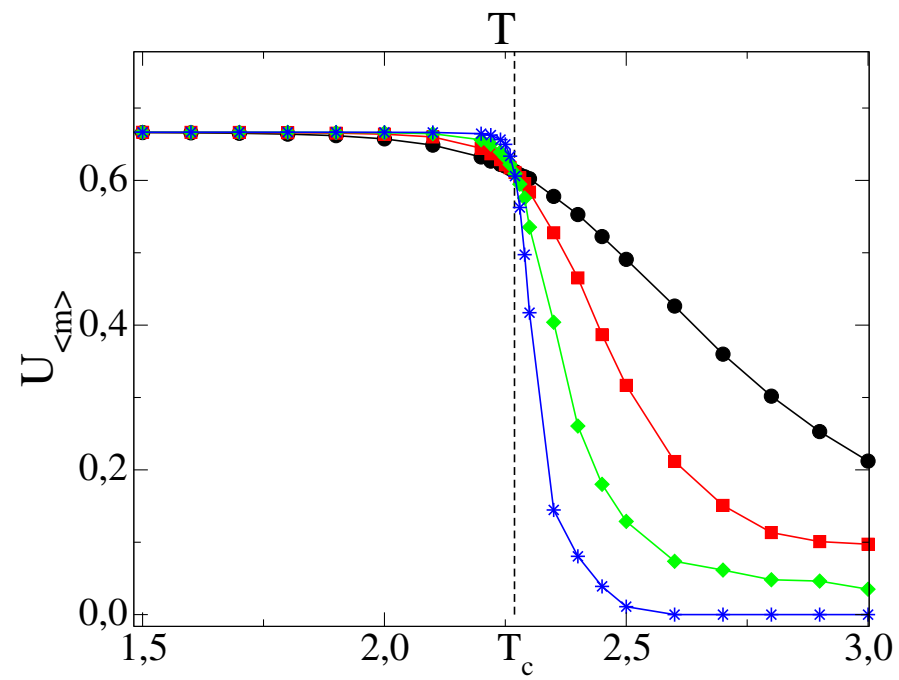

Figura 1.6: Cumulante de Binder $\left(U_{\langle m\rangle}\right)$ en función de la temperatura $(T)$ para diferentes tamaños del sistema: $N=10^{2}$ (círculos), $N=20^{2}$ (cuadrados), $N=40^{2}$ (triángulos) y $N=100^{2}$ (estrellas). La línea punteada muestra la temperatura crítica $T_{c}$ en la intersección de las curvas.

\subsubsection{Relaciones de escala}

En Mecánica Estadística, el modelo de Ising describe una transición de fase continua con un parámetro de orden escalar. Los exponentes críticos de la transición son valores universales y caracterizan las propiedades singulares de las cantidades físicas. En el modelo de Ising bidimensional, cuando nos acercamos a la temperatura crítica, todos los observables se comportan como:

$$
O(T) \propto|t|^{\alpha} .
$$




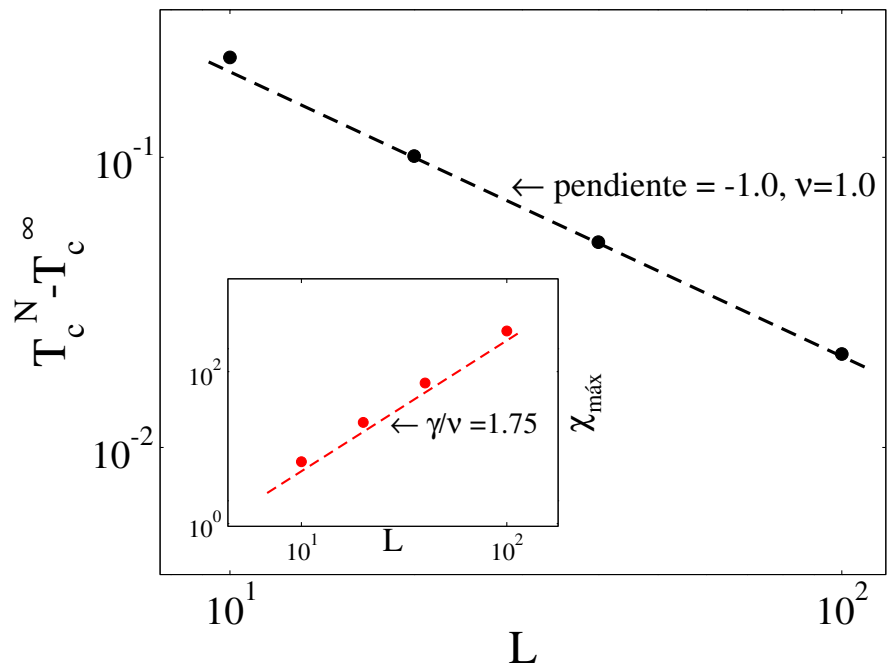

Figura 1.7: Diferencia entre la temperatura crítica obtenida por el máximo de $\chi$ $\left(T_{c}^{N}\right)$ para varios tamaños del sistema y la temperatura crítica teórica $\left(T_{c}^{\infty}=\right.$ 2.269185) en función de la longitud de un lado de la red $L$. Usando relaciones de escaleo, la pendiente de la curva representa el exponente crítico asociado con la Longitud de Correlación, $\nu=1.0$ (Fig. principal). Altura del pico de la susceptibilidad $\chi_{\text {máx }}$ vs $L$, la pendiente de la curva representa el exponente crítico asociado $\chi, \gamma=1.75$ (Fig. interna).

donde $t=\frac{T-T_{c}}{T_{c}}$ es la temperatura reducida y $\alpha$ es un exponente crítico.

Los exponentes críticos cumplen las llamadas relaciones de escala. Particularmente tenemos interés en los exponentes críticos asociados con la Susceptibilidad Magnética $\gamma$ y la Longitud de Correlación $\nu$ que mide las fluctuaciones del parámetro de orden.

El escaleo para la susceptibilidad magnética es:

$$
\chi\left(t, L^{-1}\right) \sim L^{\frac{\gamma}{\nu}} \tilde{\psi}\left(t L^{\frac{1}{\nu}}\right) .
$$

donde $\tilde{\psi}$ es la función de escala y debe ser una función analítica de su argumento. Este escaleo presenta dos predicciones: el cambio en el valor del punto crítico efectivo -definido por el máximo de $\chi^{-}$al variar $L$, que debe escalear como $L^{-\frac{1}{\nu}}$; y la altura del pico, que se comportará como $\left.L^{\frac{\gamma}{\nu}} 30\right]$.

La Fig. 1.7 muestra los resultados obtenidos en nuestras simulaciones. En la Fig. principal trazamos la diferencia entre la temperatura crítica obtenida por el máximo de $\chi\left(T_{c}^{N}\right)$ para todos los tamaños de sistema y la temperatura crítica 
teórica $\left(T_{c}^{\infty}=2.269185\right),\left(T_{c}^{N}-T_{c}^{\infty}\right)$ en función del valor de la longitud del lado de la red cuadrada $(L)$. Con la pendiente de la curva, calculamos el exponente crítico $\nu$ asociado con la longitud de correlación, y se obtuvo $\nu=1.0$, lo cual es consistente con los resultados conocidos para el Modelo de Iisng en 2D [30]. En la Fig. interna, graficamos la altura del pico de la susceptibilidad magnética $\chi_{\text {máx }}$ en función de $L$, de acuerdo con el escaleo de la Ec. (1.5), el valor del exponente crítico asociado a la Susceptibilidad Magnética $(\gamma)$ es la pendiente de la curva $\frac{\gamma}{\nu}=1.75$. Ambos exponentes críticos obtenidos en nuestras simulaciones son consistentes con la teoría [28.

\subsubsection{Coarsening en el Modelo de Ising}

La dinámica de ordenamiento del Modelo de Ising es un prototipo para procesos análogos que ocurren en muchos modelos de Dinámica Social. Para cortas escalas de tiempo, se forman dominios ordenados de tamaño pequeño, tanto positivos como negativos. La evolución posterior ocurre a través de un proceso en el cual dichos dominios crecen cada vez más (coarsening), mientras que sus características estadísticas globales permanecen sin cambios a lo largo del tiempo. Este es el fenómeno de escaleo dinámico: la morfología permanece estadísticamente igual si se reescala por la longitud típica del dominio que crece con el tiempo como

$$
\ell(t) \sim t^{1 / 2}
$$

y que es la única longitud relevante en el sistema (ver Fig. 1.8).

Macroscópicamente, la dinámica alcanza el orden por tensión superficial. La presencia de esta tensión es una consecuencia de la tendencia de cada espín a alinearse con la mayoría de sus vecinos.

Las interfases son lugares donde el sistema se separa en dos regiones diferenciadas. La formación de interfases entre dominios de magnetización opuesta tiene un costo en términos de energía y su contribución pueden minimizarse haciendo que sean lo más rectos posible. Este tipo de ordenamiento a menudo es impulsado por la curvatura y ocurre en muchos modelos sociales de formación de opinión. En el modelo de Ising la densidad de interfases $\rho$ se relaciona con la longitud típica del dominio como

$$
\rho \sim \ell^{-1}(t)
$$




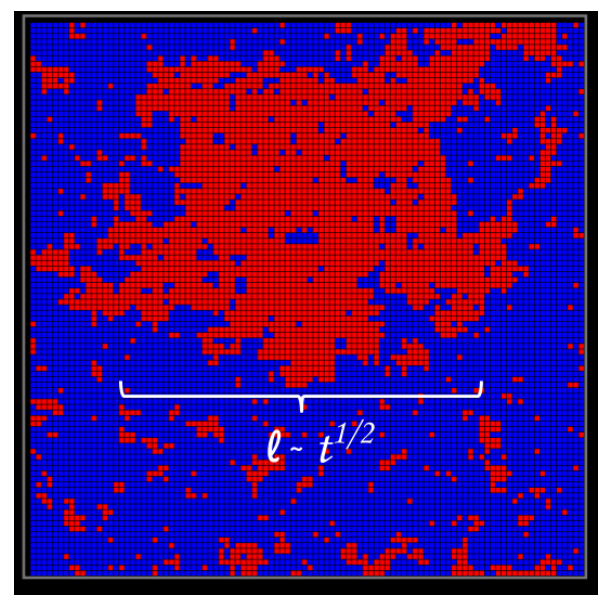

Figura 1.8: Instantánea de la dinámica del Modelo de Ising donde se visualiza la longitud típica del dominio que crece como $\ell(t) \sim t^{1 / 2}$.

lo que implica que

$$
\rho \sim t^{-1 / 2}
$$

\subsubsection{Modelo del Votante}

Uno de los modelos más simples y conocidos de opinión discreta es Modelo del Votante [1]. En este modelo, la opinión de cada agente está representada por una variable binaria que toma valores $s= \pm 1$. En cada paso de tiempo, se selecciona al azar un agente $i$ junto con uno de sus vecinos $j$ y $s_{i}$ se establece en $s_{j}$, es decir, el agente $i$ toma la opinión del vecino $j$. Esta regla de actualización implica que los agentes imitan la opinión de sus vecinos [31].

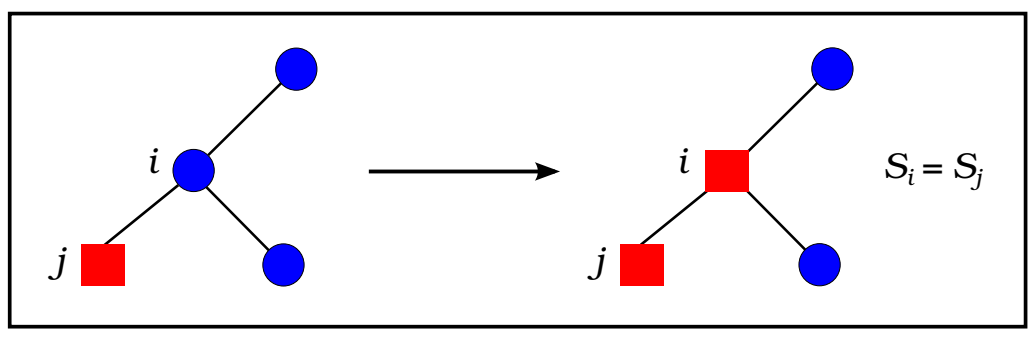

Figura 1.9: En el Modelo del Votante el agente $i$ toma la opinión de su vecino $j$. Se muestra la actualización en una Red Compleja.

En una red regular (cuadrada), podemos considerar que cada sitio de la red representa a un votante que puede tomar una de dos posturas disponibles ante 
un tema político. Estas opciones se representan como un movimiento en dirección hacia arriba $\uparrow$ o hacia abajo $\downarrow$, en analogía a un sistema de espines. Podemos pensar en los votantes como personas con autoconfianza nula que simplemente asumen la opinión de uno de sus vecinos. A partir de una condición inicial desordenada, la dinámica del votante tiende a aumentar el orden del sistema, ya que grupos de igual opinión comienzan a aparecer y es más probable que los votantes vecinos compartan esa opinión. El tamaño medio de éstos grupos (dominios) aumenta con el tiempo hasta que, para un sistema finito, solo un grupo abarca todo el sistema y decimos que la población finalmente alcanza el consenso de opinión.

Una magnitud de interés es el tiempo medio de consenso $\tau_{c}$, el cual se define como el tiempo en el que todos los nodos tienen el mismo estado, este tiempo es calculado sobre un número suficientemente grande de realizaciones. Para redes regulares finitas de $N$ sitios, $\tau_{c}$ depende de la dimensionalidad de la red. En una dimensión, el tiempo medio de consenso escalea como $\tau_{c} \sim N^{2}$, en dos dimensiones como $\tau_{c} \sim N \ln N$ y en dimensión $d>2$ como $\tau_{c} \sim N \llbracket 1$.

Para Redes Complejas, Vázquez y colaboradores [32] mostraron una expresión para este tiempo en "redes no correlacionadas", estas son redes con distribuciones de grado arbitrarias pero sin correlaciones de grado en los nodos, comenzando con una condición inicial uniforme de $N / 2$ nodos con opinión + y $N / 2$ nodos con opinión - :

$$
\tau_{c}=\frac{(\mu-1) \mu^{2}}{(\mu-2) \mu_{2}} N \ln (2) .
$$

donde $\mu_{2}$ es el segundo momento de la distribución de grado. Para una red ER $\mu_{2}=\mu(\mu+1)$ y $\tau_{c}$ resulta:

$$
\tau_{c}=\frac{(\mu-1) \mu}{(\mu-2)(\mu+1)} N \ln (2) .
$$

donde $\mu$ es el grado medio de la red.

Otra magnitud de interés es la densidad de links (enlaces) activos $\rho$ (Fig. 1.10, definida como

$$
\rho_{l a}=\frac{\text { número de links activos }}{\text { número total de links }} .
$$

Un link activo es aquel que conecta nodos con diferentes estados, verificando la condición $s_{i}+s_{j}=0$.

Para este modelo también se reprodujeron los resultados más relevantes utilizando como condición inicial una distribución uniforme de estados $+\mathrm{y}-$ sobre la 


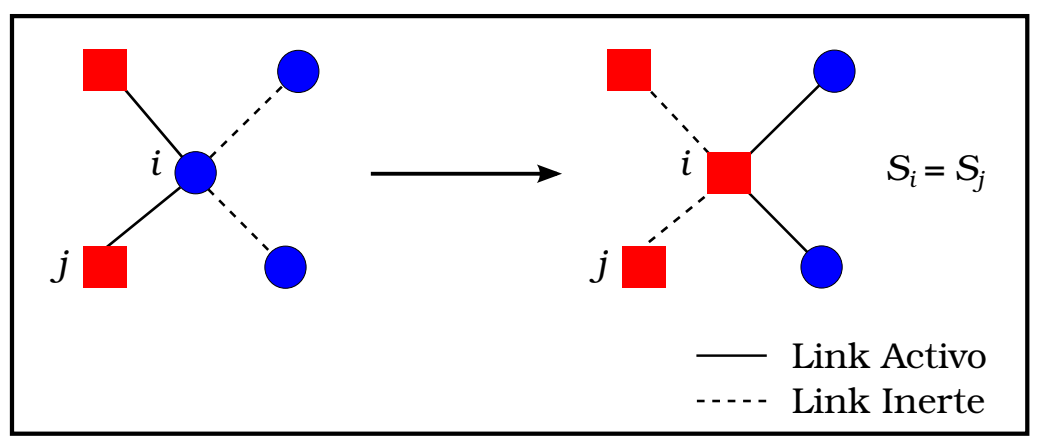

Figura 1.10: Tipos de links utilizados en el algoritmo optimizado.

red, de forma que - en promedio- hay $N / 2$ votantes en cada estado. El valor medio de la densidad de links activos $\langle\rho(t)\rangle$ sobre muchas realizaciones independientes del sistema, sigue la siguiente expresión analítica 32 :

$$
\langle\rho(t)\rangle=\frac{(\mu-2)}{2(\mu-1)}\left(1-m_{0}^{2}\right) e^{-2 t / \tau} .
$$

donde $m_{o}=0$ es la magnetización inicial y $\tau$ depende de $\mu$ como $\tau=\frac{(\mu-1) \mu^{2}}{(\mu-2) \mu_{2}} N$.

Los resultados de las simulaciones presentados en las subsecciones 1.3.2, 1.3.3 y 1.4.1, se obtuvieron mediante la utilización del Método de Monte Carlo. Este método es esencialmente un algoritmo computacional basado en la repetición de muestreos aleatorios con el fin de estimar valores medios de cantidades difíciles de calcular de manera exacta. Para más detalles ver [33].

\subsubsection{Simulación rápida: Algoritmo optimizado}

Como mencionamos al principio de esta subsección, el modelo del votante tiene una dinámica simple: un nodo y un vecino se eligen al azar y el nodo elegido copia el estado de opinión del vecino. Este proceso se repite hasta alcanzar el consenso.

Al realizar cualquier simulación, nuestro objetivo principal, además de obtener resultados interesantes que nos permitan describir el sistema en estudio, es reducir su tiempo de ejecución. En la evolución de dinámica del votante aparecen nodos inertes (nodos con vecinos que tienen el mismo estado) que ralentizan la dinámica cuando son elegidos ya que no pueden cambiar su estado. Por esta razón propusimos un algoritmo que solo tiene en cuenta los nodos activos del sistema (aquellos que tienen al menos uno de sus vecinos con estado diferente) y, por lo tanto, pueden 
cambiar de estado. Los nodos con estados diferentes están conectados por links activos. Utilizando éstas definiciones podemos acelerar la dinámica y así optimizar el tiempo de ejecución. Este algoritmo optimizado guarda relación con el Algoritmo de Gillespie 34 .

A continuación detallaremos el algoritmo original (Algoritmo 1) y el algoritmo optimizado (Algoritmo 2) para comprender más claramente el razonamiento descrito anteriormente.

\author{
Algoritmo 1 Original. \\ - Escoger un nodo "i" al azar \\ - Escoger un nodo vecino " $j "$ al azar \\ - $s_{i} \rightarrow s_{i}=s_{j}$ (se copia el estado del nodo vecino) \\ - tiempo $=$ tiempo $+1 / N$ \\ - REPETIR \\ if el número de nodos activos $\boldsymbol{a n}=0$ \\ SALIR \\ end
}

Cuando el número de nodos activos $\boldsymbol{a n}=0$, el sistema habrá llegado al consenso. Cuando no hay más links activos, el sistema queda congelado ya que no hay más cambios posibles: estado absorbente.

En la Fig. 1.10 se muestran los tipos de links utilizados en el algoritmo optimizado. En este Algoritmo, los vecinos son chequeados, ya que podrían convertirse en nodos activos (inertes) y entrar (salir) en la lista donde están contenidos.

Utilizando el Algoritmo 2 (Optimizado), simulamos el Modelo del Votante en una red Erdös Rényi. La Figura 1.11 muestra el tiempo medio de consenso $\left(\tau_{c}\right)$ como una función del tamaño del sistema $(N)$ para redes con diferentes grados medios $\mu$. A medida que $\mu$ aumenta, los resultados de la simulación se acercan a la expresión analítica Eq. 1.9 .

En la Figura 1.12 graficamos la densidad media de links activos $\langle\rho(t)\rangle$ en una red ER como función del tiempo normalizado para diferentes tamaños de sistema: $N=1000$ (círculos negros), $N=5000$ (cuadrados verdes) y $N=10000$ (diamantes 


\section{Algoritmo 2 Optimizado.}

- Recorrer toda la red chequeando si cada nodo es activo o inactivo

- Construir una lista de nodos activos y de links activos

\section{DO}

- Escoger un nodo "i" al azar de la lista de nodos activos

- Escoger un nodo vecino " $j$ " al azar

- $s_{i} \rightarrow s_{i}=s_{j}$ (se copia el estado del nodo vecino)

- Chequear si el nodo " $i$ " se convierte en inactivo para removerlo de la lista

- Chequear la actividad de los vecinos del nodo " $i ":$ nodos activos(inactivos)

$\rightarrow$ agregar(remover) a(de) la lista

- Actualizar lista de links activos

- tiempo= tiempo $+1 / \boldsymbol{a n}$

if $a n=0$

SALIR

end

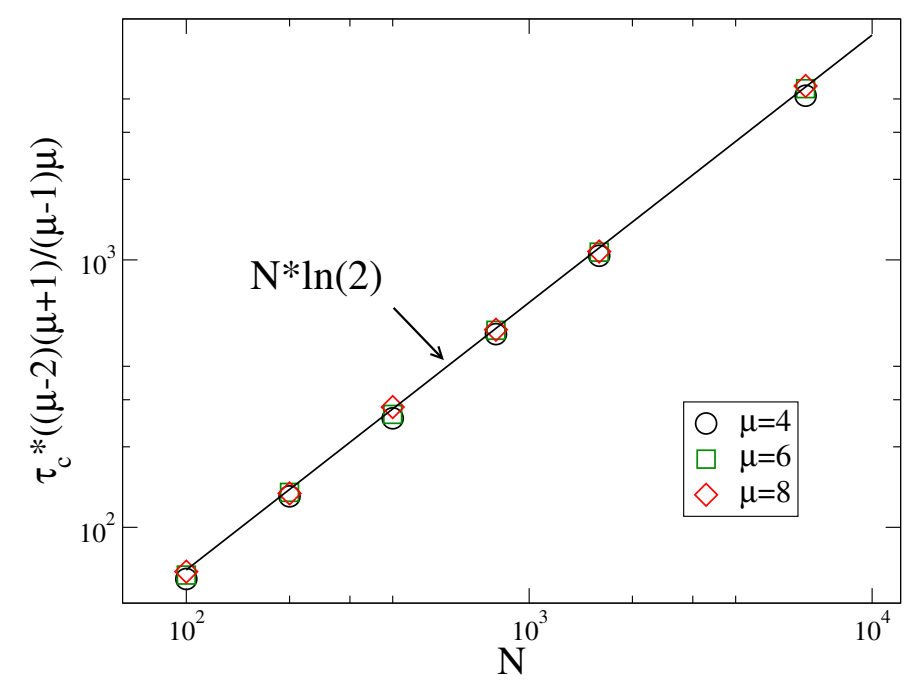

Figura 1.11: Tiempo medio de consenso $\left(\tau_{c}\right)$ vs el tamaño del sistema $(N)$. Los símbolos abiertos corresponden a 3 redes con distribuciones de grado diferentes: $\mu$ $=4$ (círculos negros), $\mu=6$ (cuadrados verdes) y $\mu=8$ (diamantes rojos). La línea sólida corresponde a la expresión analítica 1.9.

rojos). Se realizó un promedio sobre 1000 realizaciones independientes, comenzando con una distribución uniforme con magnetización $\left\langle m_{0}\right\rangle=0$. Observamos que la 


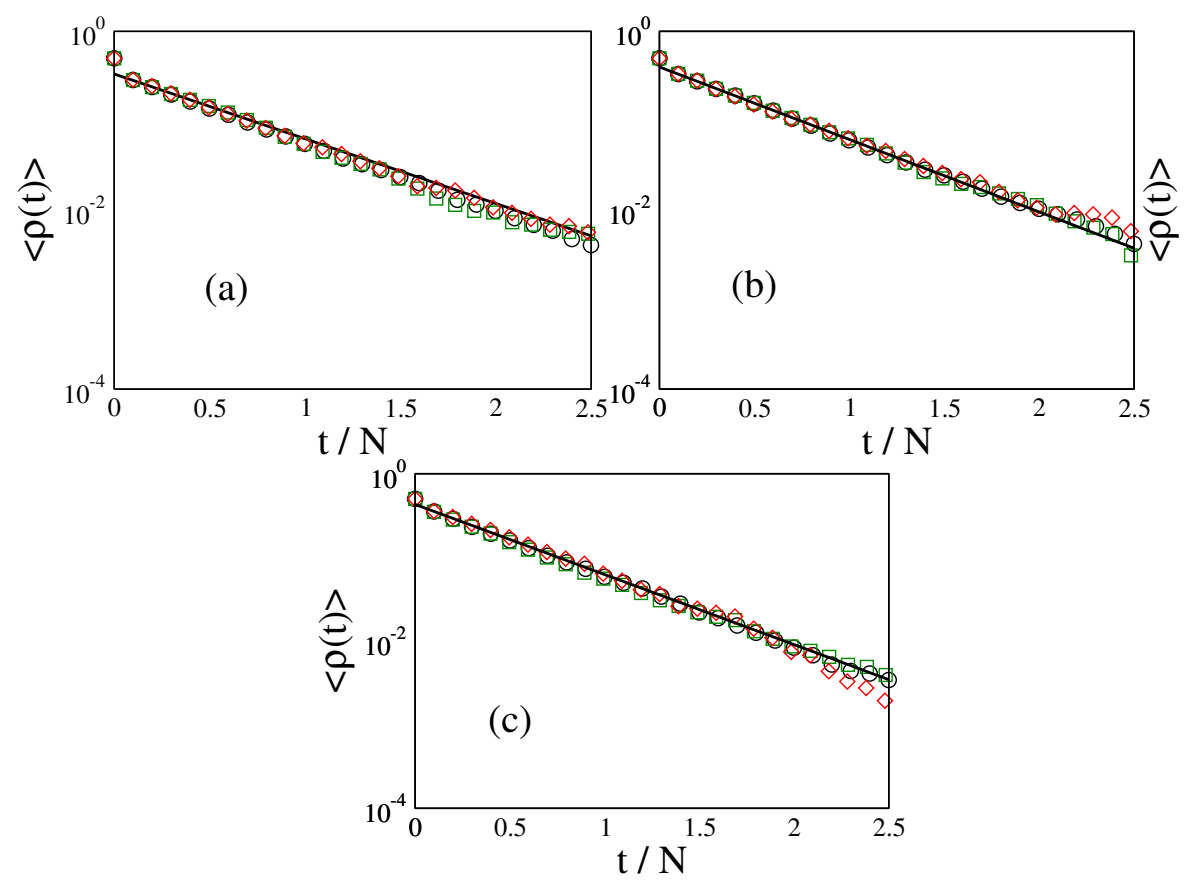

Figura 1.12: Evolución temporal de la densidad media de links activos $\langle\rho(t)\rangle$ en una red ER con diferentes grados medios: (a) $\mu=4$,(b) $\mu=6$ and (c) $\mu=8$. Los símbolos abiertos corresponden a redes con diferentes tamaños: $N=1000$ (círculos negros), $N=5000$ (cuadrados verdes) and $N=10000$ (diamantes rojos). La línea sólida representa la predicción analítica para $\langle\rho(t)\rangle 1.10$.

predicción analítica 1.10 coincide con nuestras simulaciones numéricas.

\subsubsection{Modelo M: Persuasión y Compromiso}

La influencia social tiene una gran relevancia en la formación de opiniones. En la vida cotidiana se presentan muchas situaciones en las que es necesario que un grupo comparta sus decisiones, llegando a acuerdos, o convenciendo a otros pares sobre un tema específico, para estudiar estos fenómenos se formuló el Modelo M.

El Modelo M fue propuesto por La Rocca y colaboradores [26] y explora la competencia entre dos mecanismos de formación de opinión: la persuasión y el acuerdo por compromiso. Este modelo fue analizado en campo medio en donde cada agente puede interactuar con cualquier otro agente en la población.

El proceso de persuasión puede ocurrir entre individuos con la misma orientación de opinión y tiende a reforzar sus opiniones previas. Para introducir este 
mecanismo es necesario mencionar la Teoría de Argumentos Persuasivos [35 39]. Como observó Myers en experimentos de discusión grupal [35], cuando dos personas hablan, no solo expresan sus opiniones, sino que también discuten sobre los argumentos que las respaldan. Si ya tienen la misma orientación de opinión, esta podría intensificarse cuando se persuaden unos a otros con nuevos argumentos o razones, volviéndose más extremistas en sus creencias.

Por otro lado, el acuerdo por compromiso tiende a moderar puntos de vista opuestos y fue estudiado como parte del fenómeno de Presión Social. Al respecto se argumenta que dos personas que interactúan pueden ejercer presión social para cambiar sus actitudes y conformarse mutuamente [2. En los modelos relacionados con este mecanismo [1,40 42, las opiniones están representadas por un número real entre dos valores extremos, y un par de individuos interactúan solo si su diferencia de opinión es más pequeño que un umbral dado. Las personas resuelven el conflicto al llegar a un compromiso, en el que ambas opiniones cambian en la misma cantidad para reducir su diferencia.

En el Modelo M, la opinión de cada agente está representada por un número entero $k$ en el intervalo $[-M, M](k \neq 0)$ que describe su grado de acuerdo sobre un tema político, desde totalmente en contra $(k=-M)$ hasta totalmente a favor $(k=M)$, pasando por valores intermedios. Dos agentes interactuantes con la misma orientación (positiva o negativa) refuerzan sus opiniones en una unidad y se vuelven más extremistas con una probabilidad de persuasión $p$, mientras que las opiniones de dos agentes que interactúan con orientaciones opuestas se acercan en una unidad cada uno con probabilidad de compromiso $q$.

El signo de $k$ y su valor absoluto $|k|$ indican la orientación de opinión y su intensidad, respectivamente. En un paso de tiempo de la dinámica, dos agentes $i$ y $j$ con sus estados respectivos $k_{i}$ y $k_{j}$ se seleccionan al azar para interactuar. Luego, sus estados son actualizados según sus orientaciones de opinión (ver Fig. 1.13):

- Persuasión: Si los agentes tienen la misma orientación $\left(k_{i}, k_{j}>0\right.$ o $\left.k_{i}, k_{j}<0\right)$, ocurre el mecanismo de persuasión con probabilidad $p$, donde cada agente incrementa su intensidad en una unidad y mantienen su opinión cuando $|k|=$ $M$.

- Compromiso: Si los agentes tienen orientaciones opuestas $\left(k_{i}>0\right.$ y $k_{j}<0$ o $k_{i}<0$ y $k_{j}>0$ ), ocurre el mecanismo de compromiso con probabilidad $q$, 

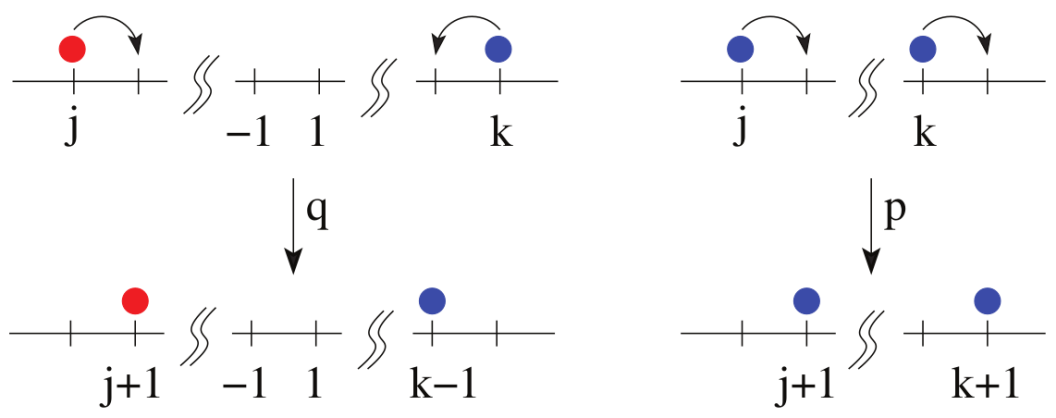

Figura 1.13: Modelo M [26]. Izquierda: Acuerdo por compromiso, dos agentes interactuantes con orientaciones opuestas se vuelven moderados. Derecha: Persuasión, dos agentes interactuantes con la misma orientación se vuelven más extremistas.

donde cada agente disminuye su intensidad en una unidad. Si ambos agentes son moderados, uno de ellos cambia su orientación al azar.

Podemos pensar en la persuasión y el compromiso como dos mecanismos en competencia que dan forma a la distribución de opiniones en la población. Mientras que las interacciones persuasivas hacen que las personas adopten opiniones extremas $M$ y $-M$ y conduzcan a la bipolarización de opiniones, los eventos de compromiso tienden a moderar las opiniones, promoviendo una distribución de opinión centralizada en torno a valores moderados 1 y -1 . Para estudiar estos fenómenos analizaremos la evolución temporal del sistema en ambos regímenes: bipolarización y centralización. Una cantidad importante a analizar es la evolución temporal del número de agentes en cada estado, que describe al sistema a nivel macroscópico.

En la Fig.1.14 se muestra la evolución temporal de cada cantidad $x_{k}(t)$, definida como la fracción de agentes en el estado $k$ al tiempo $t$, que son normalizadas en todo momento $\left(\sum_{k} x_{k}(t)=1 \forall t \geq 0\right)$. La distribución inicial de estados es uniforme, por lo que $x_{k}(t=0) \simeq 1 / 2 M$. El comportamiento cualitativo del sistema depende de la frecuencia relativa entre eventos de persuasión y compromiso, la que está controlada por la relación $r \equiv p / q$ entre las probabilidades de que ocurran estos eventos, y que recibe el nombre de parámetro de refuerzo. Por conveniencia, establecimos $p+q=1.0[p=r /(1+r)$ y $q=1 /(1+r)]$. Analizamos el comportamiento del sistema al variar $r$ para $M=5$ y $N=10^{9}$, integrando el Sistema de 

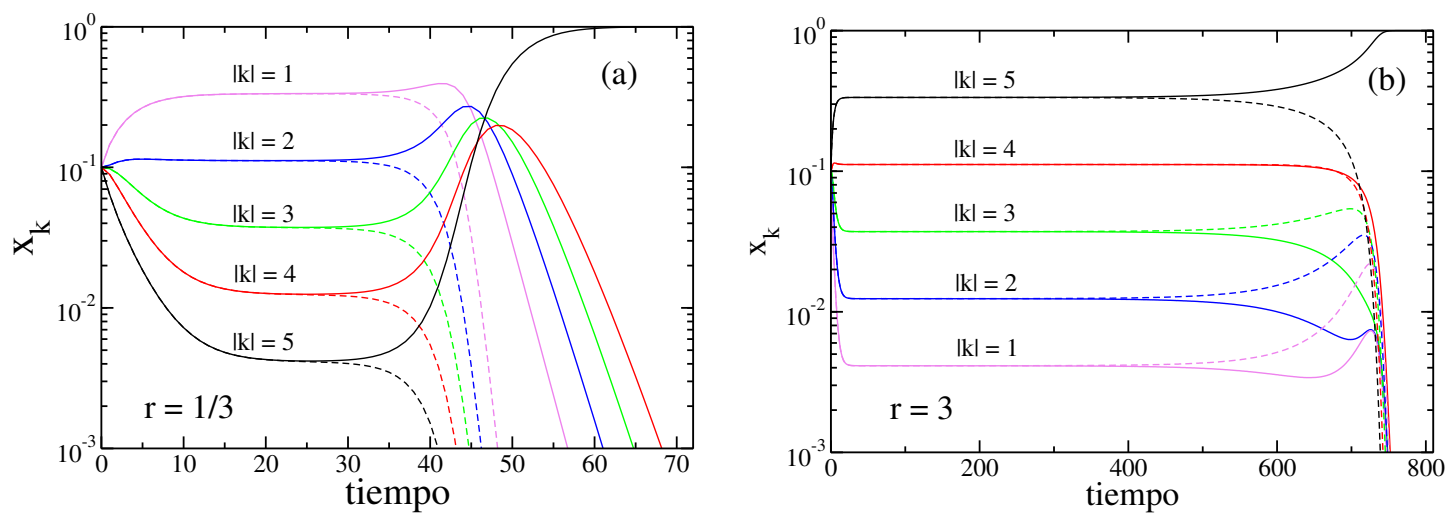

Figura 1.14: Evolución temporal de las densidades de agentes $x_{k}$ con diferentes estados de opinión $k$, para un sistema de $N=10^{9}$ agentes, $M=5$ y dos valores de $r=p / q$. (a) $x_{k}(t)$ para $r=1 / 3$ y (b) $x_{k}(t)$ para $r=3$. Las líneas sólidas (punteadas) corresponden a las opiniones positivas (negativas). Se usó una escala logarítmica en el eje $y$ para ver claramente los plateau que se forman.

Ecuaciones que lo describe $[26$ :

$$
\begin{aligned}
\frac{d x_{1}}{d t} & =2\left(x_{-1} q-x_{1} p\right) \sigma_{+}+2 q\left(x_{2}-x_{1}\right) \sigma_{-} \\
\frac{d x_{k}}{d t} & =2 p\left(x_{k-1}-x_{k}\right) \sigma_{+}+2 q\left(x_{k+1}-x_{k}\right) \sigma_{-}, \quad \text { para } 2 \leq k \leq M-1 \\
\frac{d x_{M}}{d t} & =2 p x_{M-1} \sigma_{+}+2 q x_{M} \sigma_{-}
\end{aligned}
$$

donde $\sigma_{+}=\sum_{k=1}^{M} x_{k}$ y $\sigma_{-}=\sum_{k=-1}^{-} M x_{k}$ son las densidades totales de los estados positivos y negativos respectivamente, las cuales satisfacen la relación de conservación $\sigma_{+}+\sigma_{-}=1$. Las Ecuaciones para las densidades de estados negativos se obtienen sustituyendo $k \longleftrightarrow-k$ y $\sigma_{+} \longleftrightarrow \sigma_{-}$en las ecuaciones 1.11 .

Observamos que las densidades $x_{k}$ alcanzan un valor casi constante (plateau) que depende de $k$, sin embargo, eventualmente $x_{k}$ decae a cero, excepto $x_{M}$ que se va a 1 , correspondiendo a un consenso en el estado extremista $M$. El consenso en los estados extremistas $x_{ \pm M}=1$ son estados absorbentes del sistema, por lo tanto, son los únicos estados finales posibles a largo plazo. En la Fig.1.15 se muestra la evolución temporal de cada cantidad $x_{k}(t)$ para $M=2$ y $N=10^{5}$. Los plateaus observados en la Fig.1.14 se deben al tamaño del sistema $\left(N=10^{9}\right)$, el cual es mucho más grande que el utilizado en esta simulación.

La distribución estacionaria de opiniones depende de la intensidad de refuer- 

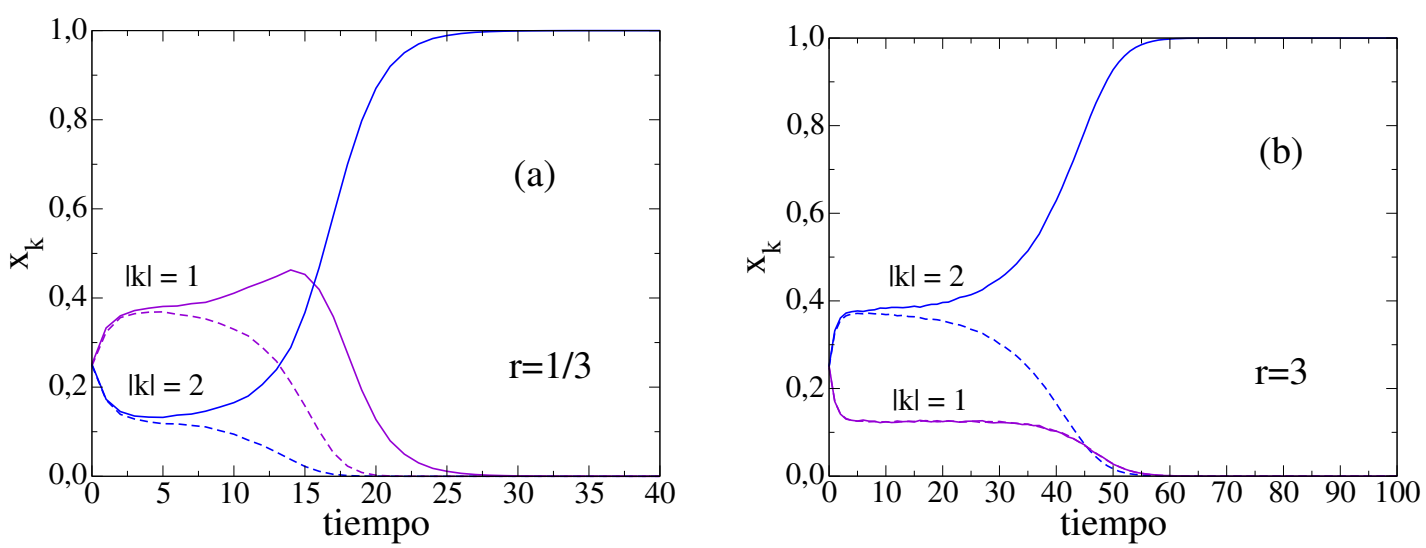

Figura 1.15: Evolución temporal de las densidades de agentes $x_{k}$ con diferentes estados de opinión $k$, para un sistema de $N=10^{5}$ agentes, $M=2$ y dos valores de $r=p / q$ obtenidos mediante simulaciones de Monte Carlo. (a) $x_{k}(t)$ para $r=1 / 3$ y (b) $x_{k}(t)$ para $r=3$. Las líneas sólidas (punteadas) corresponden a las opiniones positivas (negativas).

zo $r$. Cuando $r<1$, los eventos de compromiso ocurren más a menudo que los encuentros persuasivos, por lo que la mayoría de las opiniones de los agentes toman valores moderados $k= \pm 1$ (centralización), Fig,1.16(a). Por otro lado, cuando $r>1$, los procesos de persuasión dominan sobre los de compromiso, la mayoría de agentes se van a opiniones extremas $k \pm M$, y esto induce a la polarización de opiniones (Fig 1.16(b)). Este comportamiento es capturado mediante la expresión que describe la densidad cuasi-estacionaria de agentes en estado $k, x_{k}^{s}$ :

$$
x_{k}^{s}=\frac{1}{2}\left(\frac{1-r}{1-r^{M}}\right) r^{|k|-1} .
$$

Como hemos visto, el sistema muestra diferentes comportamientos con $r$, lo que repercute en el tiempo que le toma al sistema alcanzar el consenso extremista. En la Fig. 1.17 se muestra el tiempo medio de consenso $\tau$ como función del parámetro de refuerzo $r$. 

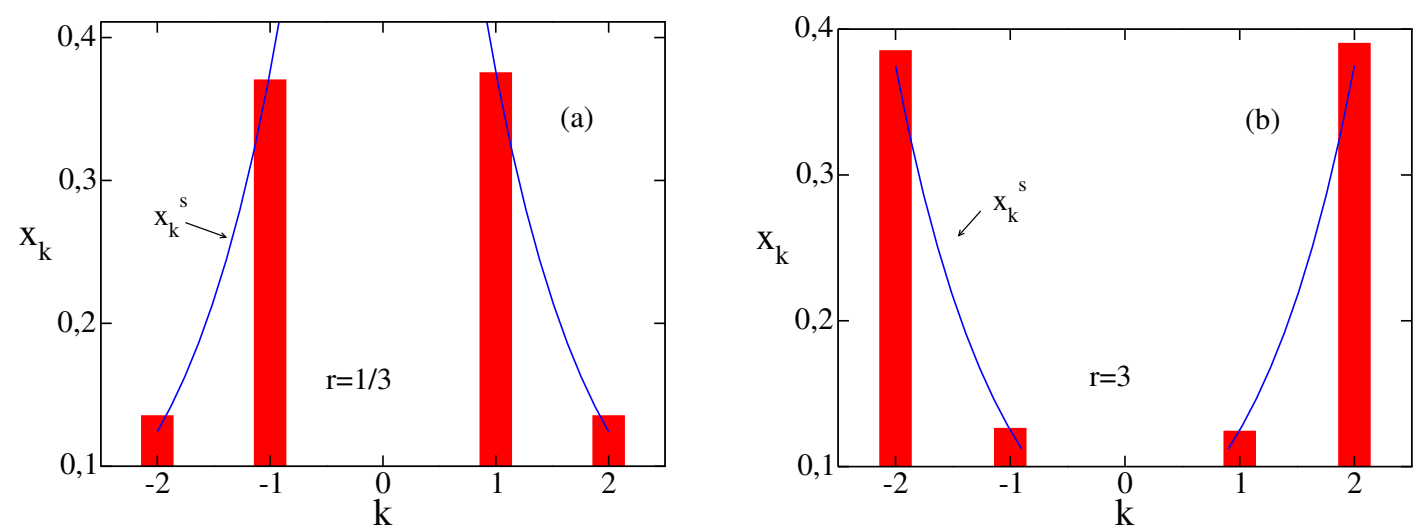

Figura 1.16: Distribución de la densidad de agentes $x_{k}$ con diferentes opiniones $k$ en el estado cuasi-estacionario, para un sistema de $N=10^{5}$ agentes, $M=2$ y dos valores de $r=p / q$ obtenidos mediante simulaciones de Monte Carlo. (a) $x_{k}(t)$ para $r=1 / 3$ a $t=5$ y (b) $x_{k}(t)$ para $r=3$ a $t=10$. Las líneas sólidas corresponden a la expresión teórica 1.12 en 26 .

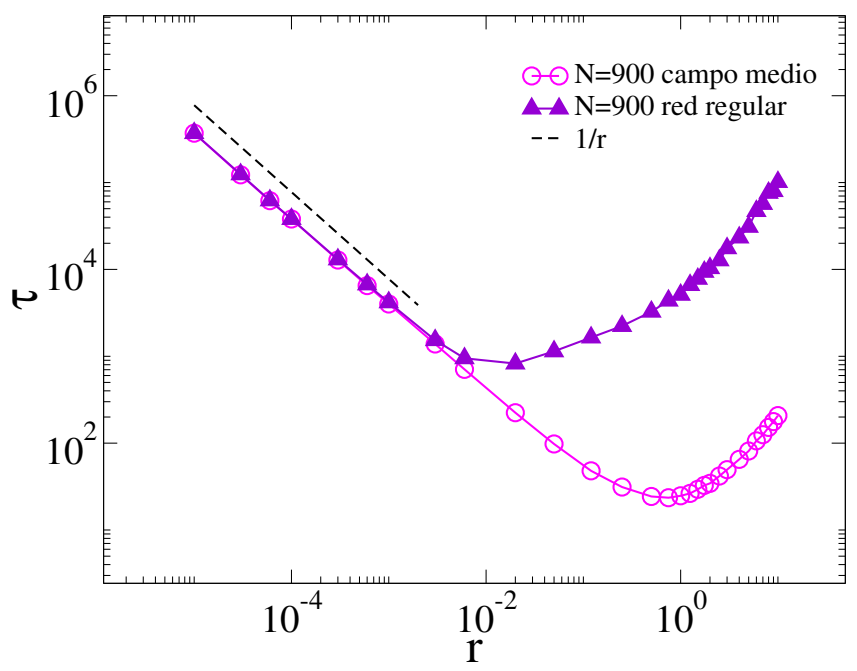

Figura 1.17: Tiempo medio de consenso $\tau$ como función de $r$ en escala doble logarítmica para $N=900$ y $M=2$. Los círculos muestran el resultado para campo medio y los triángulos para una red regular. La línea punteada muestra que para el límite de $r$ pequeño, el sistema puede describirse mediante la función $2 r^{-1}$ para cualquier valor de $M$ (no mostrado).

El tiempo medio de consenso $\tau$ muestra un comportamiento no monótono con $r$, con un mínimo alrededor de $r \simeq 0.6$. Esta tendencia nos indica dos comportamientos diferentes alrededor del mínimo, lo que hace que dividamos el análisis en dos límites ( $r$ pequeño y $r$ grande). La población alcanza el consenso más rápido 
cuando las interacciones entre agentes de la misma orientación tienen una probabilidad de éxito $p$ similar a la de los agentes de orientación opuesta $q$. Cuando $r$ es grande (Fig 1.14(b)), la mayoría de las interacciones son persuasivas y los agentes refuerzan sus posturas iniciales, lo que lleva a un estado polarizado que dura mucho tiempo. Para $r$ chico (Fig.1.14(a)), primero se induce un estado centralizado donde la mayoría de los agentes tienen opiniones moderadas, y luego la población se dirige lentamente al consenso final extremista.

En forma resumida, el modelo M incorpora dos mecanismos de formación de opinión y estudia la competencia entre ambos. Cuando los eventos de compromiso dominan, las opiniones se agrupan alrededor de los valores moderados, llevando al sistema a una centralización de opiniones. En el caso opuesto (los eventos persuasivos dominan), las opiniones se vuelven extremas, induciendo a la polarización del sistema. Estos estados son inestables y eventualmente se alcanza el consenso de opiniones extremas negativas o positivas.

\subsection{Modelos de Propagación de Epidemias}

Las enfermedades infecciosas pueden tener efectos catastróficos en la vida y el bienestar de los seres humanos. En las últimas décadas, la expectativa de vida de las personas aumentó con el surgimiento de nuevas vacunas, sin embargo se incrementó la aparición de nuevas epidemias debido a que los patrones de contacto social se fueron modificando por la evolución de los medios de transporte y el cambio en el estilo de vida de las personas lo que ha servido como nuevo escenario para la realidad epidemiológica mundial $[12]$.

Con las crecientes amenazas de enfermedades emergentes, las estrategias para controlar rápida y efectivamente los brotes son vitales para la salud pública. Los modelos epidémicos fueron propuestos para ayudar a la comprensión sobre las enfermedades infecciosas y constituyen una herramienta fundamental para los epidemiólogos y las autoridades sanitarias, puesto que permiten proyectar el número de infectados y evaluar la efectividad de diferentes estrategias tales como la vacunación y la cuarentena.

Desde Bernoulli [43], matemáticos y estadísticos han ofrecido muchas ideas prácticas sobre el control de enfermedades infecciosas. Anderson y May [12, entre otros, han ampliado estos esfuerzos en un enfoque flexible, conocido como modelado 
compartimental, para predecir la transmisión de una amplia gama de enfermedades en escalas múltiples. Uno de los primeros pasos involucrados en esta modelización consiste en identificar o separar a la población en clases, de acuerdo al estado en que se encuentren los individuos respecto de la enfermedad. Las clases más comunes son: Susceptible (S), Infectado (I) y Recuperado (R). De esta separación es que surgen los llamados Modelos Compartimentales [12.

Los modelos de este tipo son ampliamente usados por los epidemiólogos debido a que son muy útiles para predecir algunas magnitudes globales, como la evolución del número de infectados durante un período de tiempo. Dependiendo del proceso de propagación, se pueden construir distintos modelos combinando diferentes clases (o creando nuevas), los más conocidos son: el modelo SI (susceptible-infectado), el modelo SIS (susceptible-infectado-susceptible), el modelo SIR (susceptible-infectadorecuperado o removido) y el modelo SEIR (susceptible-expuesto-infectado-removido) 44].

En esta sección nos centraremos en la descripción del Proceso de Contacto y del modelo Susceptible-Infectado-Susceptible, en los cuales intervienen las mismas clases con dinámica similar pero con una actualización temporal diferente. Ambos modelos fueron utilizados en el desarrollo de esta tesis.

\subsubsection{Proceso de Contacto}

El Proceso de Contacto (CP por sus siglas en inglés), es uno de los modelos más simples de propagación de epidemias y fue propuesto por T.E. Harris en 1974 [29]. Se dice que el CP es el "Modelo Ising" para el estudio de la transiciones de fase de sistemas fuera del equilibrio. Este modelo fue diseñado para describir algunas infecciones, por ejemplo las del resfriado común y la gripe, ya que no confieren ninguna inmunidad a largo plazo. Tales infecciones no dan inmunización al recuperarse de la infección, y los individuos vuelven a ser susceptibles.

Para describir la dinámica del CP se tienen individuos con 2 estados: Susceptible (S) e Infectado (I). Cada individuo infectado pasará a estado susceptible con una probabilidad de recuperación $\mu$ o tratará de infectar a algún vecino con probabilidad $\beta$. Para reducir el número de parámetros del modelo, muchas veces se toma $\mu=1-\beta$. Este parámetro externo $\beta$ se varía con el fin de estudiar la densidad de individuos infectados/susceptibles en el tiempo. 


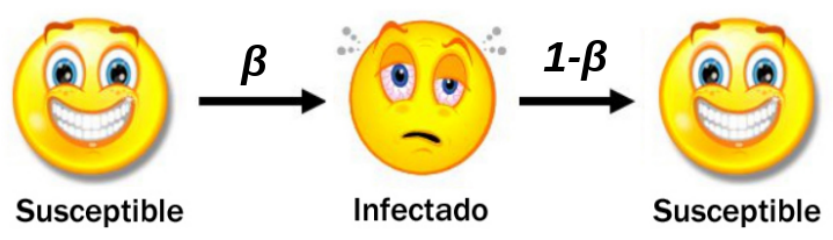

Figura 1.18: Proceso de Contacto: Los individuos pueden estar en estado susceptible (S) ó infectado (I) y cambiar su estado según la probabilidad de infección $\beta$.

Como se mencionó anteriormente, una de las cantidades relevantes en el estudio de esta dinámica es la densidad de individuos infectados $\rho(t)$ en el tiempo. La variación de esta densidad en campo medio (interacción todos con todos) se puede escribir como:

$$
\frac{d \rho}{d t}=\beta \rho(1-\rho)-(1-\beta) \rho
$$

El primer término de la Ec.1.13 $(\beta \rho(1-\rho)$ es un término de ganancia representado como un producto de probabilidades independientes: $\rho$ es la probabilidad de eligir un individuo infectado y $(1-\rho)$ es la probabilidad de elegir un vecino susceptible que se contagia con probabilidad $\beta$. El segundo término de la ecuación es un término de pérdida en el cual con probabilidad $\rho$ se escoge un agente infectado que se recupera con probabilidad $(1-\beta)$. Esta ecuación tiene 2 estados estacionarios encontrados al hacer $\frac{d \rho}{d t}=0$,

$$
\begin{aligned}
& \rho_{1}^{\text {stat }}=0, \\
& \rho_{2}^{\text {stat }}=\frac{2 \beta-1}{\beta} .
\end{aligned}
$$

Y la solución general de la Ec. 1.13 viene dada por:

$$
\rho(t)=\frac{2 \beta-1}{\beta} \frac{\rho_{0}}{\rho_{0}-\left(\rho_{0}-\frac{2 \beta-1}{\beta}\right) e^{-\left(\frac{2 \beta-1}{1-\beta}\right) t}} .
$$

Donde $\rho_{0}$ es la densidad inicial de individuos infectados.

Cuando el parámetro $\beta$ aumenta, existe un valor crítico $\beta_{c}=1 / 2$ que define una transición entre una fase sana donde $\rho=\rho_{1}^{\text {stat }}=0$ y una fase endémica donde $\rho=\rho_{2}^{\text {stat }}>0$ en el cual se identifica el "punto crítico" y la expresión para $\rho(t)$ es de la forma:

$$
\rho(t) \propto t^{-1}
$$




\subsubsection{CP en dos tipos de red: Grafo Completo vs Red Erdös-Rényi}

La Ec.(1.14) describe la evolución temporal de la fracción de individuos infectados $\rho(t)$ en campo medio. Para comparar el Monte Carlo de la dinámica del CP con la solución de la Ec. 1.14 es necesario realizar la simulación en una red de tipo Grafo Completo [45, en la que todos los nodos están conectados entre si y la cantidad de conexiones que tiene cada nodo es $N-1$.

En la Fig.1.19 se observa que la simulación numérica (símbolos) coincide con la solución analítica dada por la Ec.(1.14) para $\rho(t)$ (línea sólida), para varios valores de $\beta$. En esta Figura se muestra el promedio de la densidad de individuos infectados $\langle\rho(t)\rangle$ sobre 1000 realizaciones independientes de la dinámica.

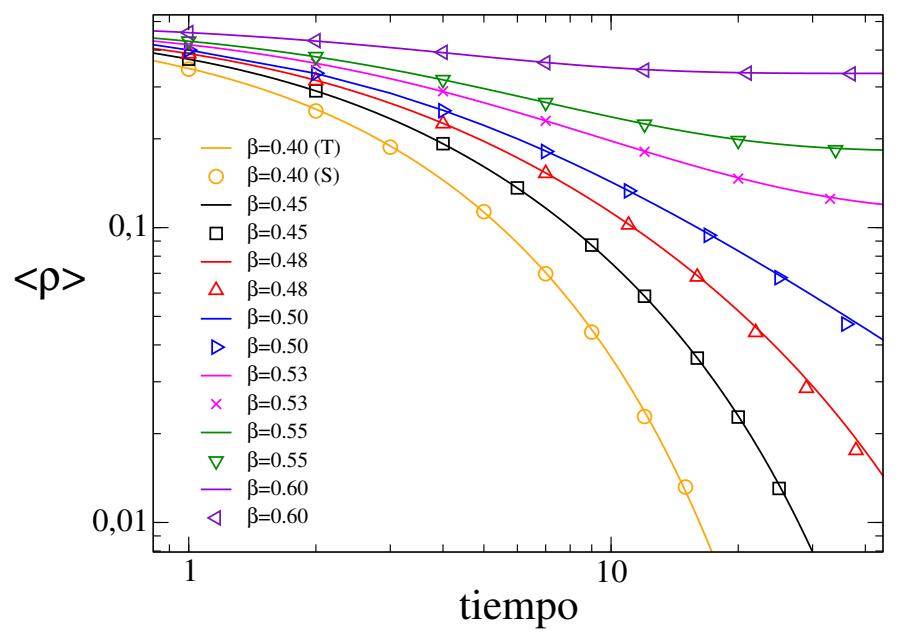

Figura 1.19: Densidad de individuos infectados $\rho$ en función del tiempo. La línea sólida corresponde a la solución analítica y los símbolos son los resultados de la simulación numérica para $N=1000$ nodos y varios valores de la probabilidad de infección $\beta$.

En el punto crítico, el sistema sigue una ley de Potencias y para un Grafo Completo se localiza en $\beta_{c}=0.5$. En Redes Complejas, el valor del punto crítico depende del grado medio de la red $\mu$ [46]. En una red tipo Erdös-Rényi con $\mu=10$, el punto crítico se encuentra en $\beta_{c}=0.527$ (Fig,1.20).

Como se mencionó en secciones anteriores y como parte del entrenamiento, se buscó nuevamente mejorar los tiempos en nuestras simulaciones, para esto se construyó un código optimizado, el cual sólo toma en cuenta los nodos infectados dentro 


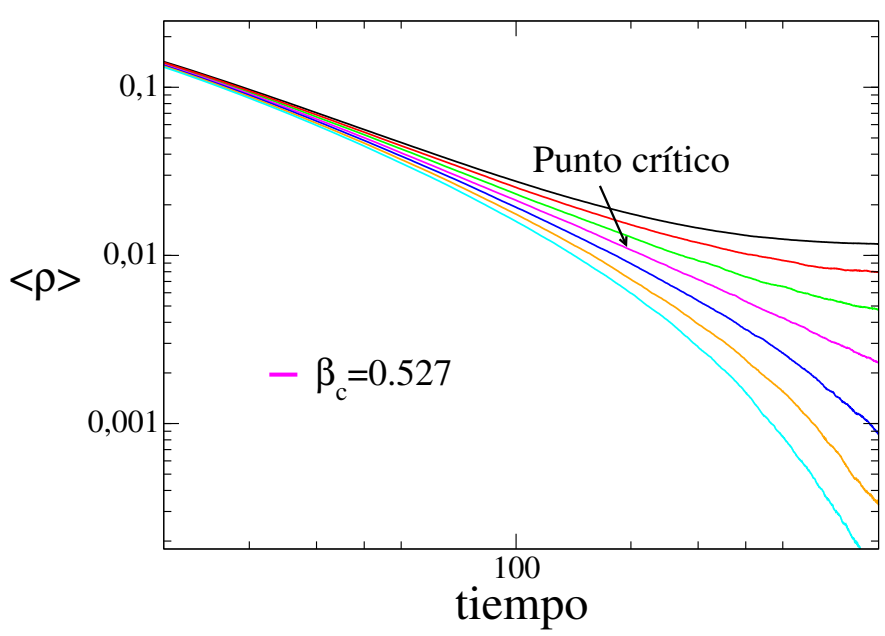

Figura 1.20: Densidad media de individuos infectados $\rho$ en función del tiempo. Código sin optimizar para $N=10^{6}$ nodos, la línea color magenta representa el punto crítico de la probabilidad de infección $\beta$ para una red ER cuyo valor es $\beta_{c}=0.527$, con $\mu=10$.

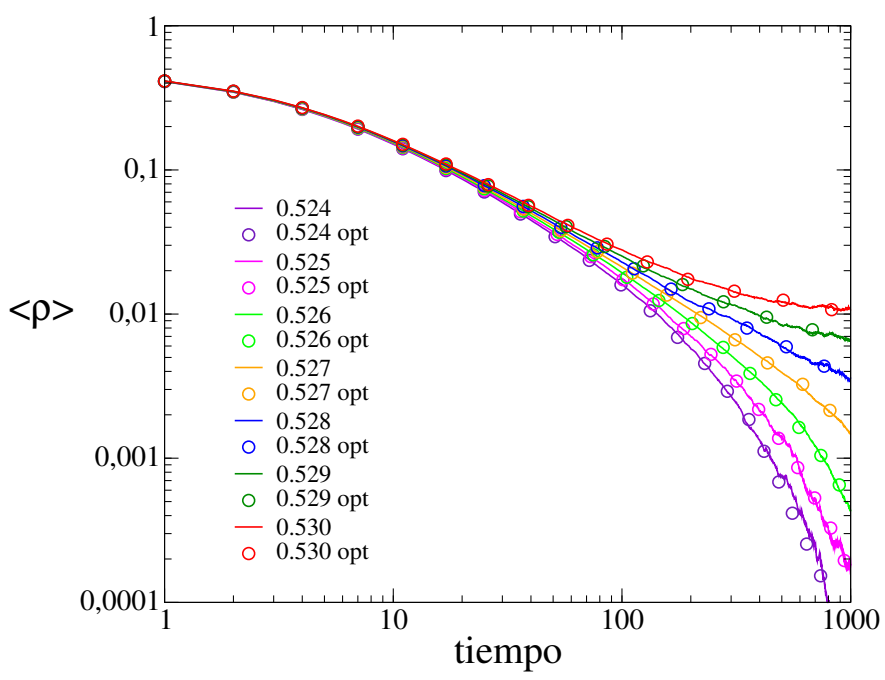

Figura 1.21: Densidad media de individuos infectados $\rho$ vs $t$. Código sin optimizar (línea sólida) y optimizado (círculos abiertos) para $N=10^{5}$ nodos.

de la red. En un paso de la dinámica, se escoge aleatoriamente un individuo de la lista de nodos infectados y se aumenta el tiempo en $1 /$ (cantidad de infectados).

En la Fig 1.21 observamos la coincidencia de los códigos optimizado (círculos abiertos) y sin optimizar (línea sólida), graficando la densidad de individuos infectados $\rho$ en el tiempo para valores cercanos al punto crítico $\beta_{c}$ y éste, inclusive, para una red ER de $N=10^{5}$ nodos y grado medio $\mu=10$. 


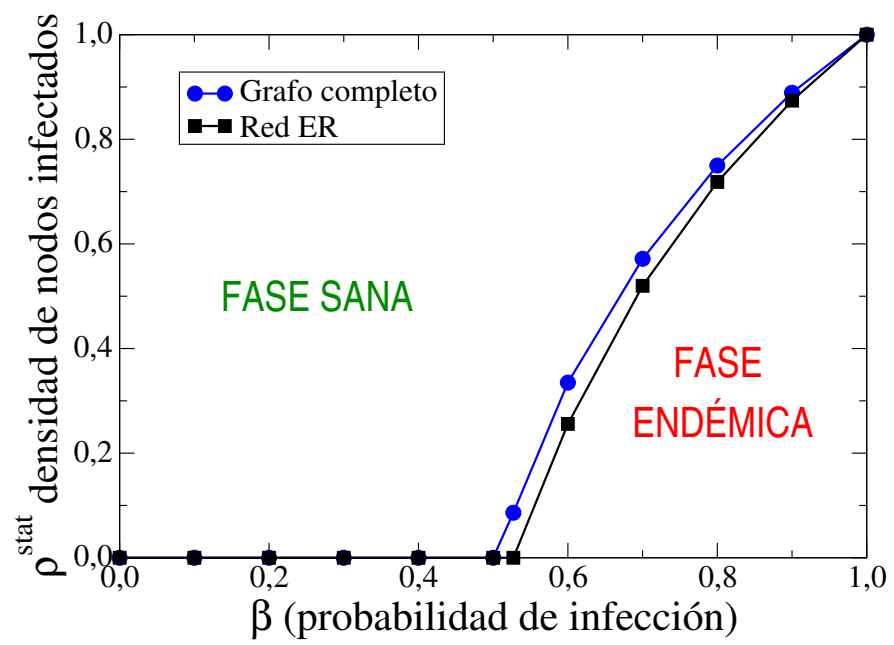

Figura 1.22: Densidad estacionaria de nodos infectados $\rho^{\text {stat }}$ vs $\beta$. El punto crítico varía según el tipo de red utilizada: Grafo completo (círculos), Red ER (cuadrados) con $\mu=10$.

En la Fig. 1.22 se muestra la densidad estacionaria de nodos infectados $\rho^{\text {stat }}$ en función de la probabilidad de infección $\beta$, podemos observar que $\beta_{c}$ varía según el tipo de red utilizada, y que a partir de este valor se identifican las fases existentes en el modelo: Sana y Endémica.

\subsubsection{CP vs. Susceptible-Infectado-Susceptible: SIS}

El modelo Susceptible-Infectado-Susceptible (SIS) también es un modelo de enfermedad endémica análogo al Proceso de Contacto explicado en la sección anterior 1.4.1. La diferencia entre ambos modelos está en la actualización de la dinámica, en el CP la actualización se realiza a tiempo discreto mientras que en el SIS es a tiempo continuo, lo que se conoce como actualización en paralelo: en cada paso de tiempo diferencial $d t$ todos los agentes actualizan sus estados.

El SIS pertenece a los modelos endémicos del tipo compartimental [12], los cuales subdividen a la población en las poblaciones por estado de enfermedad. Estos modelos asumen que una vez infectados, los agentes son inmediatamente infecciosos y permanecen así hasta que se recuperan. En un paso de tiempo $d t$ cada agente infectado trata de contagiar a cada uno de sus vecinos susceptibles con probabilidad $\beta d t$, luego se recupera con probabilidad $\mu d t$. 
En los últimos años, los procesos de propagación de información se modelan utilizando analogías con modelos epidémicos. En recientes trabajos 47 50 acoplan ambos procesos aprovechando la similitud en las reglas de estas dinámicas. 


\section{Capítulo 2}

\section{La formación de opiniones en redes cuadradas}

El proceso de formación de opiniones en una sociedad involucra varios mecanismos complejos. Una amplia investigación sobre este asunto muestra que la mayoría de los patrones empíricos de opinión se asemejan a los de la bipolarización, en lugar de a los de consenso [51]. El fenómeno de bipolarización se define como la formación de dos grupos con opiniones antagónicas que intensifican sus diferencias con el tiempo, y donde las posiciones entre los dos extremos del espectro de opinión están cada vez menos ocupadas 38.

En la misma línea, Bonacich y Lu [52] notaron que muchos modelos muestran cómo los grupos llegan al consenso, pero no hay modelos generalmente aceptados de cómo los grupos se polarizan o cómo dos grupos pueden volverse cada vez más diferentes y posiblemente hostiles. Algunos modelos que combinan influencia social positiva y negativa 53 55] conducen a una distribución de opinión bimodal que podría explicar la bipolarización, sin embargo, la influencia negativa no está totalmente respaldada por la evidencia empírica.

Basados en trabajos anteriores [37], Mäs y Flache propusieron en [38, 39] un mecanismo alternativo que combina el fenómeno de homofilia 56,57 con la "Teoría de Argumentos Persuasivos" (PAT, por sus siglas en inglés) 35, 36, 58, que da lugar a la bipolarización sin la suposición de influencia negativa. Estos autores también realizaron experimentos de discusión en grupo para probar la validez del modelo teórico. La idea es que, debido a la homofilia, un individuo tiende a interactuar y hablar con un compañero que tiene la misma orientación de opinión en un tema determinado, como por ejemplo estar a favor del matrimonio entre personas del mismo sexo. Entonces, la PAT sugiere que las dos personas que interactúan son propensas a intercambiar diferentes argumentos que respaldan sus posiciones, y 
así pueden proporcionar nuevos argumentos o razones que refuercen su opinión inicial. Esto podría intensificar los puntos de vista de los individuos y hacerlos más extremos en sus creencias. Motivado por este trabajo, La Rocca y colaboradores 26] introdujeron recientemente un modelo que incorpora el mecanismo de homofilia y persuasión de una manera simple, y que es capaz de generar niveles deseados de bipolarización. Este modelo fue descrito en la sección 1.3.3 del Capítulo 1 y nos referiremos a éste como el "Modelo M" a partir de ahora.

En el Modelo M, la opinión de cada agente está representada por un número entero $k$ limitado en el intervalo $[-M, M](k \neq 0)$ que describe su grado de acuerdo sobre un tema político, desde totalmente en contra $(k=-M)$ hasta totalmente a favor $(k=M)$. Cada agente puede interactuar con cualquier otro agente en la población, lo que corresponde a una aproximación de campo medio (MF) o interacción de "todos con todos". Dos agentes interactuantes con la misma orientación (positiva o negativa) refuerzan sus opiniones en una unidad y se vuelven más extremistas con una probabilidad de persuasión $p$, mientras que las opiniones de dos agentes que interactúan con orientaciones opuestas se acercan en dos unidades con probabilidad de compromiso q. En este trabajo [26], se muestra que el comportamiento del modelo depende de la frecuencia relativa entre interacciones de agentes con la misma orientación (persuasión) y agentes con orientación opuesta (compromiso), determinadas por la relación $r=p / q$. Cuando los eventos persuasivos dominan sobre los eventos de compromiso, las opiniones se dirigen hacia valores extremos $k=-M$ y $k=M$, lo que induce la coexistencia de opiniones extremas o bipolarización. En el caso opuesto, cuando los eventos de compromiso dominan sobre los eventos de persuasión, las opiniones se agrupan alrededor de los valores moderados $k=-1$ y $k=1$ y llevan a la centralización. Además, se observó que los estados estacionarios de bipolarización y centralización son inestables, donde una pequeña asimetría de opinión es suficiente para llevar a la población a un consenso rápido en una de las dos opiniones extremas. Si bien estos resultados corresponden a la versión de campo medio del modelo $\mathrm{M}$, las consecuencias de la competencia entre persuasión y compromiso no se habían explorado en poblaciones con interacciones que tienen lugar en el espacio o en topologías complejas.

En este capítulo, describiremos en detalle la dinámica del modelo $\mathrm{M}$ en una red cuadrada de dos dimensiones $(2 D)$, para el caso más simple y no trivial $M=2$. Nuestra meta es investigar los efectos de los mecanismos de persuasión y compromiso en una población de agentes con interacciones a primeros vecinos (corto alcance), 
en contraste con la interacción de "todos con todos" del caso de campo medio. En particular, apuntamos a explorar cómo la topología espacial en $2 D$ afecta la estabilidad de los estados polarizados y centralizados. También buscamos comprender las propiedades básicas de la aproximación hacia el consenso extremista.

Los mecanismos de persuasión y compromiso se han implementado en varios trabajos para modelar la formación de opiniones en poblaciones que interactúan. Por un lado, la persuasión se ha introducido recientemente en algunos modelos basados en agentes [59 62]. En [59,60], la persuasión se entiende como el grado de autoconvicción que tiene una persona, que toma en cuenta la influencia de otros y su propia opinión al tomar una decisión. Los autores en 61 introdujeron un modelo en el que cada individuo puede tener una de dos opiniones opuestas o estar indeciso, y cada una de estas tres opciones están determinadas por su persuasión o grado de convicción sobre el tema en cuestión, representadas por un número real en un intervalo de persuasión. En otro trabajo se estudió un modelo donde la persuasión tiene lugar entre agentes de orientación opuesta [62].

Por otro lado, el proceso de compromiso fue inicialmente estudiado en modelos con opiniones continuas e interacciones umbrales [40,41, y la estabilidad de la distribución de la opinión bimodal fue probada bajo la influencia del ruido [63]. Algunos modelos de votantes con múltiples estados 64 67] han incorporado una regla similar a un compromiso que utiliza un mecanismo de refuerzo por el cual los agentes cambian de orientación solo después de tener múltiples interacciones con agentes de orientación opuesta. Por ejemplo, Castelló et al. 64 estudió un modelo de lenguaje de tres estados donde cada agente podría hablar uno de dos idiomas posibles ( $\mathrm{A}$ o $\mathrm{B}$ ) o ser un bilingüe $(\mathrm{AB})$. Un agente monolingüe A podía convertirse en bilingüe $\mathrm{AB}$ al interactuar con un agente que habla el idioma opuesto (agente $\mathrm{B}$ o agente $\mathrm{AB}$ ). Estos autores investigaron todo el proceso de ordenamiento en el sistema para redes cuadradas y redes de pequeño mundo, así como el tiempo medio de consenso asociado a cada topología. Un análisis de estabilidad de este modelo 68 reveló que el predominio de un idioma es mejorado por la conectividad de la red, y que este efecto es incluso más fuerte en redes cuadradas. Más recientemente, Volovik y Redner 65] estudiaron un modelo del votante con cuatro estados, en el cual cada agente puede elegir entre dos posibles opiniones y, además, puede tener dos niveles de compromiso con la opinión (confiada e insegura). Un votante seguro que interactúa con un agente de una opinión diferente se vuelve menos comprometido (inseguro), pero mantiene su opinión. Sin embargo, un votante inseguro 
puede cambiar su opinión al interactuar con un agente de una opinión diferente. En otro trabajo [66] Dall'Asta y Galla realizaron un estudio numérico y analítico de las propiedades de coarsening en los modelos de votantes con muchos estados intermedios en retículas (redes cuadradas) [67], que tienen reglas de interacción similares a las de los trabajos [64,65] descritos anteriormente. Ellos mostraron que la adición de estados intermedios al modelo del votante de 2 estados (VM) [5] restaura una tensión superficial efectiva. Es importante mencionar que todos estos modelos carecen del mecanismo de refuerzo de opiniones inducido por las interacciones de la misma orientación que caracteriza al Modelo M.

\subsection{El modelo $M$ en una red cuadrada}

Consideraremos la dinámica de formación de opiniones del modelo propuesto por La Rocca et al. 26] sobre una red cuadrada de $N=L^{2}$ sitios, donde $L$ es el tamaño de cada lado de la red. Cada sitio está ocupado por un agente que puede interactuar con sus cuatro vecinos más cercanos, y puede tomar uno de los cuatro estados posibles de opinión $k=-2,-1,1$ o 2 que representan su posición sobre un tema político, desde un extremo negativo $k=-2$ (un extremista negativo), a un extremo positivo $k=2$ (un extremista positivo), pasando por valores intermedios $k=-1,1$ (un moderado). El signo de $k$ y su valor absoluto $|k|$ indican la orientación de opinión y su intensidad, respectivamente. En un paso de tiempo de la dinámica de longitud $\Delta t=2 / N$, dos agentes primeros vecinos $i$ y $j$ con sus estados respectivos $k_{i}$ y $k_{j}$ se seleccionan al azar para interactuar. Luego, sus estados son actualizados según sus orientaciones de opinión (ver Fig. 2.1):

- Persuasión [Figs.2.1(a) y 2.1(b)]: Si los agentes tienen la misma orientación $\left(k_{i}, k_{j}>0\right.$ o $\left.k_{i}, k_{j}<0\right)$, ocurre el mecanismo de persuasión con probabilidad p. Un agente incrementa su intensidad en una unidad si es moderado $(|k|=1)$, o mantiene su opinión si es extremista $(|k|=2)$.

- Compromiso [Figs.2.1(c) y 2.1(d)]: Si los agentes tienen orientaciones opuestas $\left(k_{i}>0\right.$ y $k_{j}<0$ ó $k_{i}<0$ y $\left.k_{j}>0\right)$, ocurre el mecanismo de compromiso con probabilidad $q$. Si ambos agentes son extremistas $\left(\left|k_{i}\right|=\left|k_{j}\right|=2\right)$ sus intensidades tendrán un decremento en una unidad. Si un agente es extremista y el otro es moderado $|k|=1$, el extremista disminuye su intensidad en una 
unidad mientras que el moderado cambia de orientación. Si ambos agentes son moderados, uno de ellos cambia su orientación al azar.

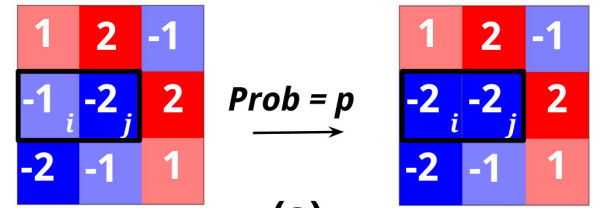

(a)

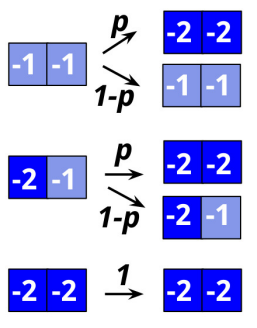

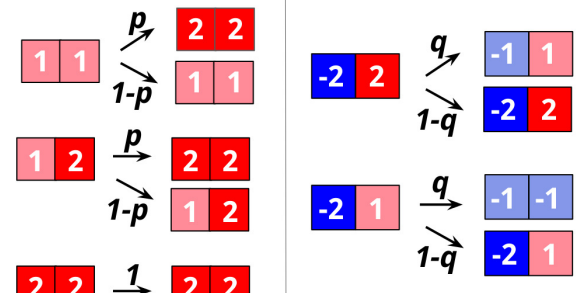

(b)

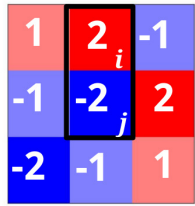

Prob $=q$

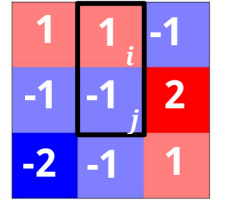

(c)

(d)

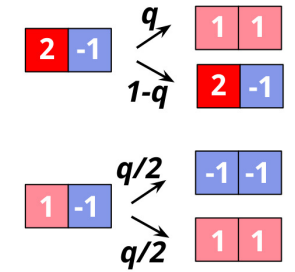

Figura 2.1: Actualizaciones de los dos mecanismos del Modelo M en una red cuadrada. Panel (a) y (b) Persuasión: En (a), un agente negativo moderado $i$ con opinión $k_{i}=-1$ se vuelve extremista $\left(k_{i}=-1 \rightarrow k_{i}=-2\right)$ con probabilidad $p$ interactuando con uno de sus vecinos, el agente $j$, que tiene opinión extrema $k_{j}=-2$. El panel (b) muestra todos los posibles eventos de persuasión en donde dos agentes vecinos con la misma orientación de opinión refuerzan sus opiniones y se vuelven extremistas. Paneles (c) y (d) Compromiso: En (c), dos vecinos interactuantes con opiniones opuestas y extremas se convierten en moderados con probabilidad $q\left(k_{i}=2 \rightarrow k_{i}=1\right.$ y $\left.k_{j}=-2 \rightarrow k_{j}=-1\right)$. El panel (d) muestra todos los posibles eventos de compromiso en donde dos vecinos con orientaciones opuestas se convierten en moderados.

Podemos pensar en la persuasión y el compromiso como dos mecanismos en competencia que dan forma a la distribución de opiniones en la población. Mientras que las interacciones persuasivas hacen que las personas adopten opiniones extremas 2 y -2 y conducen a la bipolarización de opiniones, los eventos de compromiso tienden a moderar las opiniones, promoviendo una distribución de opinión centralizada en torno a valores moderados 1 y -1 . Para estudiar estos fenómenos analizaremos la evolución temporal del sistema y exploraremos la dinámica de coarsening en ambos regímenes: bipolarización y centralización. 


\section{tiempo}
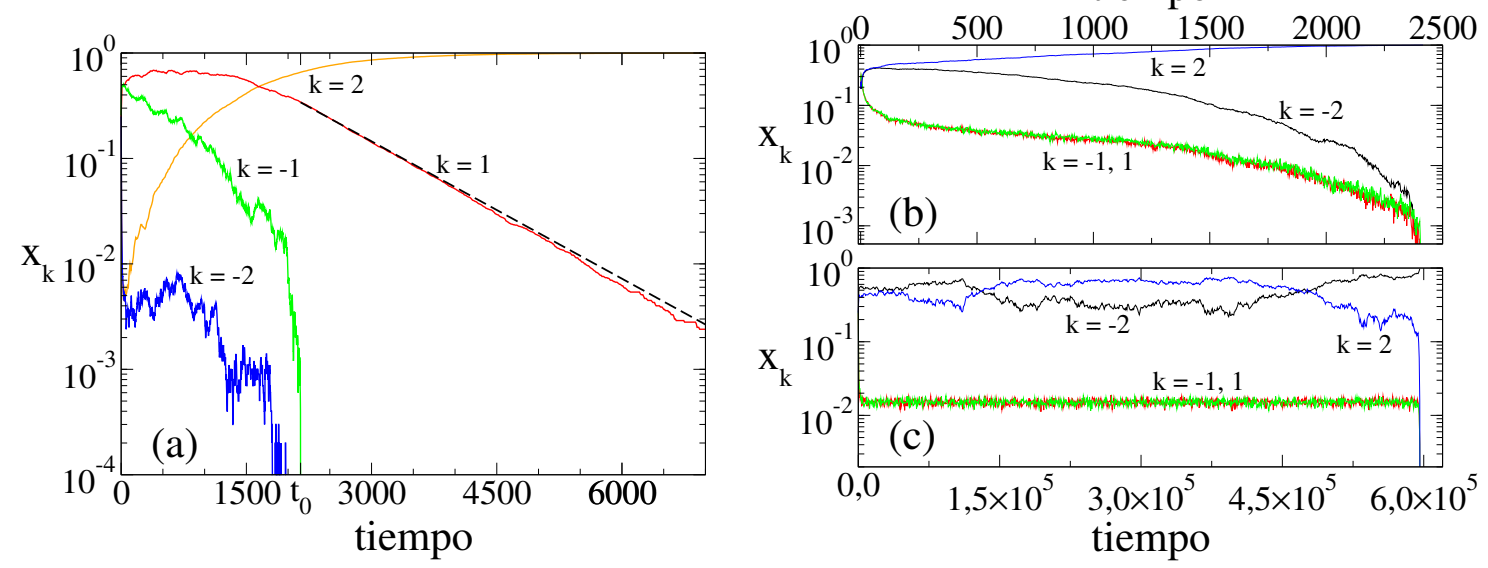

Figura 2.2: Evolución temporal de las densidades de agentes $x_{k}$ con diferentes estados de opinión $k$ en realizaciones individuales de la dinámica, para un sistema de $N=10^{4}$ agentes y dos valores de $r=p / q$. (a) $x_{k}(t)$ para $r=10^{-3}$. La línea punteada corresponde a la expresión $0.34 e^{-r(t-2150)}$ de la Ec. 2.2. Paneles (b) y (c) muestran la evolución de $x_{k}(t)$ para $r=1 / 3$ en realizaciones de tipo 1 y tipo 2 , respectivamente.

\subsection{Dinámica de coarsening}

Comenzamos el análisis numérico del modelo estudiando la evolución temporal del número de agentes en cada estado, que describen el sistema a un nivel macroscópico. Para eso, realizamos simulaciones de Monte Carlo de la dinámica descrita en la sección 2.1 donde se midieron las cantidades $x_{k}(t)(k=-2,-1,1,2)$, definidas como la fracción de agentes en el estado $k$ en el tiempo $t$, que son normalizadas en todo momento $\left(\sum_{k} x_{k}(t)=1 \forall t \geq 0\right)$. Inicialmente, cada agente adopta uno de los cuatro estados posibles con igual probabilidad 1/4. El comportamiento cualitativo del sistema depende de la frecuencia relativa entre eventos de persuasión y compromiso, que está controlada por la relación $r \equiv p / q$ entre las probabilidades de que ocurran estos eventos. Por conveniencia, establecimos $p+q=1.0$ $[p=r /(1+r)$ y $q=1 /(1+r)]$ y analizamos el sistema cuando $r$ varía. En la Fig. 2.2 mostramos la evolución de las densidades $x_{k}$ para realizaciones individuales de la dinámica para un sistema de tamaño $N=10^{4}$, y dos valores diferentes de $r$.

En la realización para un $r$ muy pequeño $\left(r=10^{-3}\right)$ [Fig. 2.2 (a)] las interacciones de compromiso son mucho más frecuentes que las interacciones persuasivas $(q \gg p)$, impulsando las opiniones de la mayoría de los agentes hacia valores mode- 
rados durante una etapa inicial $(t \lesssim 500)$ en la que $x_{1}$ y $x_{-1}$ son mucho más grandes que $x_{2}$ y $x_{-2}$. Esto corresponde a una centralización de opiniones. Luego, en el instante $t_{0}=2150$, los estados negativos -1 y -2 desaparecen y la densidad $x_{1}$ decae exponencialmente a cero, mientras que $x_{2}$ se acerca exponencialmente a 1 . Una vez que $x_{2}$ es igual a 1 el sistema no puede evolucionar más (estado absorbente), que en este caso corresponde a un consenso de opinión extremista positiva. En general, el estado final del sistema siempre es consenso en algún estado extremista, es decir, todos agentes con una opinión $2\left(x_{2}=1\right)$ o todos con una opinión $-2\left(x_{-2}=1\right)$. Se puede obtener una idea de esta aproximación exponencial hacia el consenso utilizando una aproximación de campo medio. Esto corresponde a la versión MF del modelo M para $r$ pequeños estudiados en [26]. Después de un tiempo $t_{0}$ solo los estados positivos 1 y 2 sobreviven en el sistema $\left(x_{1}(t)+x_{2}(t)=1\right)$, y por lo tanto la dinámica solo se ve impulsada por eventos persuasivos que conducen lentamente a todos los agentes al estado 2 con una probabilidad muy pequeña $p=r /(1+r) \simeq r$ en el límite $r \ll 1$. Luego, la variación promedio de $x_{1}$ en un solo paso de longitud $\Delta t=2 / N$ está dada por

$$
\frac{d x_{1}}{d t}=\frac{\Delta x_{1}}{\Delta t}=-\frac{p x_{1}^{2} \frac{2}{N}}{2 / N}-\frac{p 2 x_{1} x_{2} \frac{1}{N}}{2 / N}=-p x_{1} \quad \text { para } t \geq t_{0} .
$$

El primer término de la ecuación (2.1) describe la interacción entre dos agentes de estado 1 que realizarán su transición al estado 2 con probabilidad $p$, mientras que el segundo término toma en cuenta la transición al estado 2 para un único agente que estaba en estado 1 e interactúa con otro agente en estado 2. La solución de la ecuación (2.1) es

$$
x_{1}(t)=x_{1}\left(t_{0}\right) e^{-r\left(t-t_{0}\right)} \quad \text { para } t \geq t_{0}
$$

donde hemos usado $r$ como un valor aproximado para $p$. La expresión de la ecuación (2.2) para $x_{1}$ se grafica en la figura 2.2 (a) (línea punteada) usando $r=10^{-3} \mathrm{y}$ la condición inicial $x_{1}\left(t_{0}=2150\right) \simeq 0.34$ extraída de la curva $x_{1}(t)$. La coincidencia obtenida con las simulaciones muestra que la dinámica en una red cuadrada para un valor pequeño de $r$ puede describirse con la teoría de campo medio MF.

En las realizaciones con $r=1 / 3$ [Figs. 2.2 (b) y 2.2 (c)] están representadas las interacciones persuasivas, las cuales son más frecuentes que en el caso anterior, pero menos frecuentes que las interacciones de compromiso. Estas interacciones parecen suficientes para hacer que la mayoría de los agentes adopten estados extremos 2 y -2 , y por lo tanto $x_{2}$ y $x_{-2}$ son mayores que $x_{1}$ y $x_{-1}$ para todo tiempo. Esto corresponde a un estado polarizado donde la población de agentes se divide en dos 
grupos de tamaño similar que mantienen opiniones extremas y opuestas. También observamos que en la realización del panel (b) el sistema alcanza el consenso en un estado extremista 2 al tiempo $t \simeq 2400$, mientras que en la realización del panel (c) un consenso extremista en estado -2 se logra en un tiempo mucho más largo $t \simeq 6 \times 10^{5}$. Estos ejemplos corresponden a dos tipos diferentes de realizaciones observadas en las simulaciones. En las realizaciones de tipo 1 [panel (b)] la simetría inicial entre los estados positivos y negativos se rompe a tiempos cortos y el sistema alcanza rápidamente el consenso, donde las densidades $x_{1}$ y $x_{-1}$ decaen a cero y $x_{2}$ o $x_{-2}$ se aproximan a 1 . En las realizaciones de tipo 2 [panel (c)] el sistema cae en un estado metaestable de larga duración donde $x_{1}$ y $x_{-1}$ fluctúan alrededor de un valor estacionario durante mucho tiempo hasta que caen a cero junto con $x_{2}$. Este estado metaestable dura un tiempo mucho más largo que el observado en la versión MF del modelo 26. Esto significa que la coexistencia de opiniones podría ser muy estable cuando las interacciones son restringidas a los primeros vecinos en una red cuadrada, aumentando la estabilidad de la bipolarización de opiniones.

Para investigar el origen de los diferentes comportamientos descritos anteriormente estudiamos las propiedades de coarsening del sistema por medio de la densidad de interfases $\rho$, definida como la densidad de enlaces entre vecinos en diferentes estados 64,66]. En la Fig. 2.3 se muestra la evolución temporal de $\rho$ para realizaciones individuales con $r=10^{-4}$ [panel (a)] y $r=1 / 3$ [panel (b)], acompañadas con instantáneas del estado del sistema para diferentes tiempos y para cada tipo de realización. Se observa la formación de dominios de igual opinión con diferentes características. Para $r=10^{-4}$ [Fig. 2.3 (a)], la gran frecuencia de eventos de compromiso en comparación con los eventos persuasivos conduce a casi todos los agentes hacia estados moderados, lo que produce la formación temprana de grandes dominios compuestos por agentes con estados 1 o -1 , con unos pocos extremistas dispersos (snapshot abajo a la izquierda). Durante esta etapa, la dinámica de la interfase entre 1 y -1 sigue la del Modelo del Votante. Esto explica la forma ruidosa observada en la interfase que caracteriza el coarsening sin tensión superficial del VM [69]. Los dominios crecen lentamente hasta que casi todos los agentes, excepto algunos extremistas, adoptan el mismo estado moderado (indicado por el estado 1 en el snapshot) y $\rho$ alcanza un mínimo. Esto corresponde al comienzo de la etapa persuasiva discutida anteriormente, durante la cual los agentes moderados se vuelven extremistas. Luego, la relajación al consenso sigue el decaimiento exponencial de MF $\rho \simeq x_{1}\left(1-x_{1}\right) \sim e^{-r t}$ de la ecuación (2.2) (líneas discontinuas). 

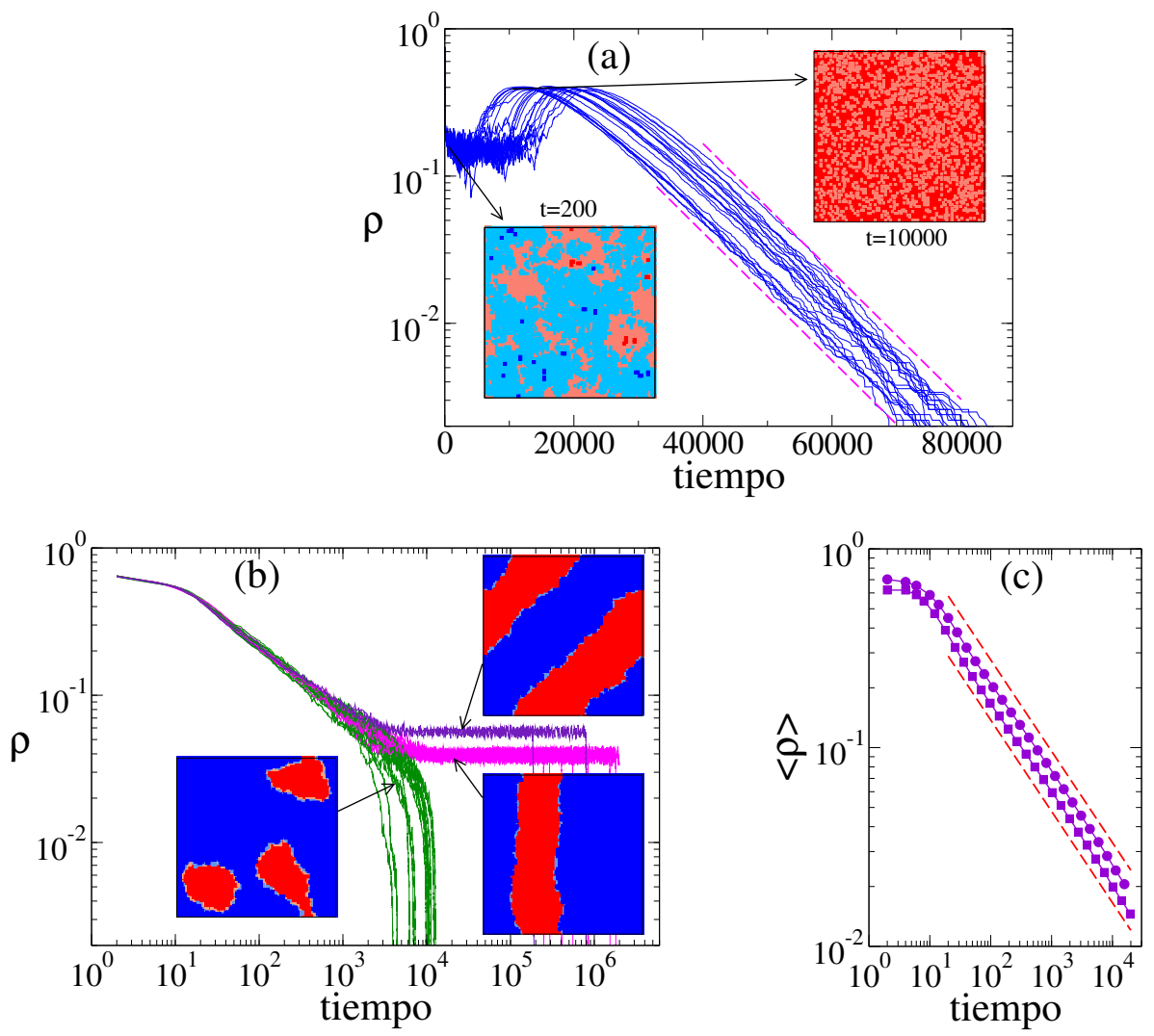

Figura 2.3: Evolución temporal de la densidad de interfase $\rho$ en realizaciones individuales, para un sistema de tamaño $N=10^{4}$ con $r=10^{-4}$ (a) y $r=1 / 3$ (b). Los snapshots de la red muestran los patrones espaciales de las opiniones a diferentes tiempos y para diferentes tipos de realizaciones. Panel (a): Snapshot de abajo a la izquierda, corresponde a una centralización de opiniones alrededor de los valores moderados $k=-1$ y $k=1$, mientras que el snapshot de arriba a la derecha muestra que todas las opiniones son positivas y la persuasión se hace presente. Las líneas punteadas tienen un valor para la pendiente de $r=10^{-4}$. Panel (b): El snapshot de abajo a la izquierda corresponde a realizaciones que alcanzan rápidamente el consenso (tipo 1), mientras que los snapshots de abajo y arriba a la derecha representan realizaciones de tipo 2 , donde el sistema queda atrapado en estados bandas de larga duración antes de alcanzar el consenso. Panel (c): densidad promedio de interfase $\langle\rho\rangle$ (círculos) y densidad promedio de estados moderados (cuadrados) en función del tiempo para un sistema de tamaño $N=300^{2}$. El promedio fue realizado sobre $10^{4}$ realizaciones. Las líneas punteadas tienen una pendiente de -0.46 . 
Esta dinámica es muy diferente a la observada en modelos de votantes con estados múltiples 64 66, donde los agentes con estados intermedios (moderados) se colocan en los bordes entre dominios de estados extremos y forman interfases bastante suaves. Este último fenómeno ocurre para $r=1 / 3$ [Fig. 2.3(b)], donde los estados moderados 1 y -1 se encuentran en la interfase entre dominios de estados 2 y -2 . Esto se observa en la Fig. 2.3 (c) donde mostramos la evolución temporal del valor promedio de $\rho$ y la densidad promedio de estados moderados (intermedios) $x_{1}+x_{-1}$. Vemos que $\langle\rho\rangle$ y $\left\langle x_{1}+x_{-1}\right\rangle$ decaen como $t^{-0.46}$, lo que indica que la dinámica de la interfase está correlacionada con la de los estados moderados. El comportamiento $\langle\rho\rangle \sim t^{-0.46}$ es consistente con el coarsening algebraico encontrado en modelos de votantes con estados intermedios [64, 66]. Como se demostró en [66, la adición de estados intermedios a los 2 estados del VM cambia las propiedades de ordenamiento del sistema, desde un coarsening impulsado por el ruido de la interfase (observado en el VM), a un coarsening con tensión superficial, observado en modelos con uno o varios estados intermedios. El exponente característico 0.46 es compatible con el exponente 0.5 asociado al crecimiento de dominios por curvatura observados en Modelos de Ising Cinéticos 70,71.

Otra observación de la Fig. 2.3 (b) está relacionada con los diferentes tipos de realizaciones, cuya dinámica de interfase explica el comportamiento temporal de las densidades moderadas $x_{1}$ y $x_{-1}$ observadas en las figuras 2.2 (b) y 2.2 (c). La evolución inicial de $\rho$ en todas las realizaciones sigue un decaimiento tipo ley de potencia descrito anteriormente, pero luego se dividen en dos grupos principales. El grupo de realizaciones que decaen rápido, llamados tambien de corta vida, corresponde a la Fig. 2.2 (b), en la cual los dominios se encogen y desaparecen hasta que un gran dominio extremista cubre todo el espacio (snapshot abajo a la izquierda). El grupo de realizaciones que caen en un estado metaestable de larga duración, donde se observan franjas horizontales o verticales (snapshot de abajo a la derecha) o franjas diagonales (arriba a la derecha), corresponde a la Fig. 2.2 (c). En estas dinámicas de estados metaestables, la densidad de interfase $\rho$ fluctúa alrededor de un valor estacionario hasta que una fluctuación de tamaño finito lleva al sistema a uno de los estados absorbentes $(\rho=0)$. El largo plateau observado para $\rho$ muestra que el estado polarizado es mucho más estable en redes cuadradas que en MF [26]. Como estudiaremos con más detalle en la sección 2.4, este comportamiento se debe a la difusión lenta de las interfases entre estas bandas, que finalmente se encuentran y aniquilan, llevando al sistema al consenso. Las bandas 
diagonales se caracterizan por un valor estacionario de $\rho$ que es aproximadamente $\sqrt{2}$ veces más grande que el valor correspondiente para bandas horizontales o verticales. También vale la pena mencionar que aunque estas bandas diagonales no se mencionan en modelos relacionados 64, 65, 72, es probablemente porque es muy poco probable que se formen (alrededor de 3 por ciento del tiempo en nuestras simulaciones), esperamos ver estas franjas diagonales en todos estos modelos con coarsening tipo Ising 73$]$.

\subsection{Tiempos de consenso}

Como mostramos en la sección 2.2, el modelo M tiene dos estados absorbentes correspondientes a los dos consensos extremistas. Una magnitud de interés en estos modelos es el tiempo medio para alcanzar el consenso de opinión $\tau$. En la Fig. 2.4 (a) presentamos resultados de simulaciones numéricas de $\tau$ como función de $r$ para tres tamaños de red diferentes $N$. Cada punto en la gráfica corresponde a un promedio de $10^{4}$ realizaciones independientes con condición inicial uniforme. Vemos que $\tau$ es no monótono con $r$, tomando valores muy elevados para $r$ pequeños y grandes. También observamos que $\tau$ aumenta con $N$ y que el aumento es mucho más rápido para $r$ grandes, lo que sugiere dos escalas diferentes alrededor del mínimo. De hecho, los paneles (b) y (c) de la Fig. 2.4 muestran el colapso de los datos para valores pequeños y grandes de $r$ cuando las curvas se dividen por $\ln N$ y $N^{1.64}$, respectivamente. La escala logarítmica de $\tau$ con $N$ en el límite cuando $r$ es pequeño se puede obtener del comportamiento de la densidad $x_{1}$ dada por la Ec. 2.2. Primero notamos que el decaimiento exponencial de $x_{1}$ con el tiempo se mantiene para $r \ll 1$ y $N$ (no mostrado), y que el tiempo $t_{0}(r, N)$ en el que la etapa persuasiva comienza, varía tanto con $r$ como $N$. Para derivar una expresión para $\tau$ hacemos dos suposiciones. Primero, esperamos que la distribución de los estados a $t_{0}(r, N)$ tenga un máximo en $k=1$, es decir, $x_{1}\left(t_{0}\right) \simeq 1$ y $x_{2}\left(t_{0}\right) \simeq 0$. De hecho, hemos comprobado que $x_{1}\left(t_{0}\right)$ se aproxima a 1.0 cuando $r$ disminuye $\left(x_{1}\left(t_{0}\right) \simeq 0.34\right.$ para $r=10^{-3}$, mientras que $x_{1}\left(t_{0}\right) \simeq 0.7$ para $\left.r=10^{-4}\right)$. En segundo lugar, suponemos que se llega a un consenso cuando hay menos de un agente en el estado 1, lo que conduce a la condición $x_{1}=1 / N$ en el tiempo $\tau$. Luego, despejando $\tau$ de la relación $1 / N \simeq e^{-r\left[t-t_{0}(r, N)\right]}$ llegamos a la aproximación $\tau \simeq t_{0}(r, N)+r^{-1} \ln N$. El segundo término está asociado a la duración de la etapa persuasiva que domina 


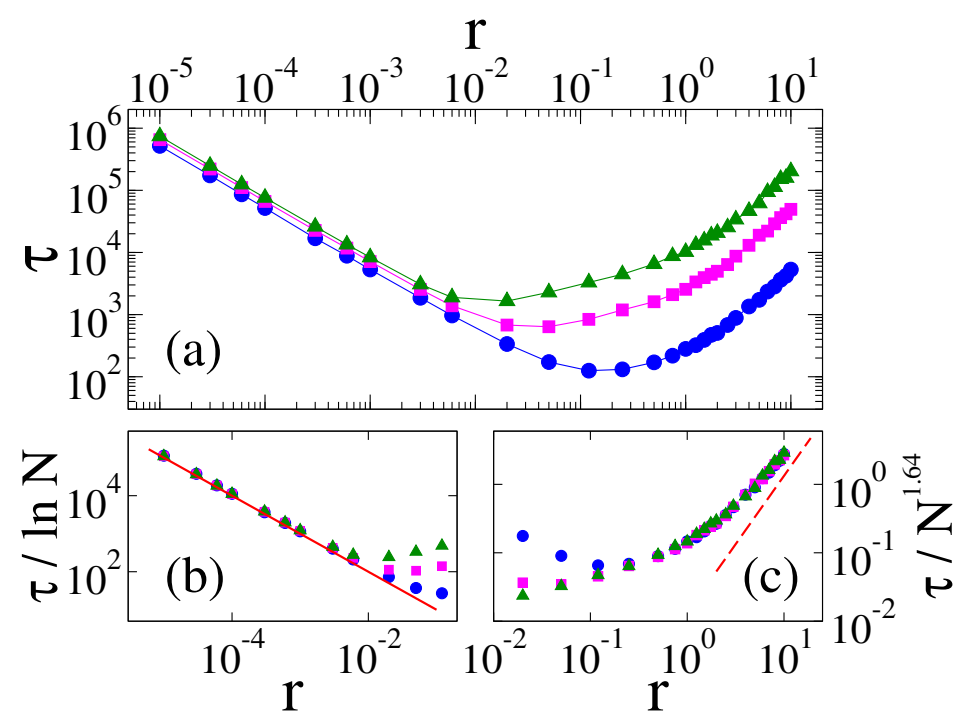

Figura 2.4: (a) Tiempo medio de consenso $\tau$ como función de $r$ en escala doble logarítmica para diferentes tamaños de red: $N=100$ (círculos), $N=400$ (cuadrados) y $N=900$ (triángulos). Paneles (b) y (c) muestran el colapso para valores pequeños y grandes de $r$, respectivamente. La línea sólida en (b) corresponde a la aproximación analítica $\tau \simeq r^{-1} \ln N$ de la Ec. (2.3), mientras que la línea punteada en (c) tiene pendiente 2 .

en el límite para $r$ pequeño y, por lo tanto, $\tau$ puede ser aproximado como

$$
\tau \simeq \frac{\ln N}{r} \quad \text { para } r \ll 1
$$

Observamos en la Fig. 2.4 (b) que la expresión analítica Ec. 2.3 representada por la línea continua, coincide con los datos numéricos, mostrando la divergencia $1 / r$ de $\tau$ en el límite de $r \rightarrow 0$.

El comportamiento de ley de potencia $\tau \sim N^{1.64}$ usado para colapsar los datos para los valores grandes de $r$ [ver Fig. 2.4 (c)] se obtuvo ejecutando simulaciones para $r=1 / 3$ y varios tamaños de sistema. Los resultados se muestran en la Fig. 2.6 con círculos vacíos, donde también trazamos una línea sólida con pendiente 1.64 que sirve como guía visual, y que corresponde al mejor ajuste de los datos. Los puntos de la Fig. 2.4 (c) colapsan en una sola curva que parece acercarse al comportamiento cuadrático $r^{2}$ cuando $r$ se vuelve grande (línea punteada), y esto sorprendentemente concuerda con lo predicho por la expresión MF $\tau_{\mathrm{MF}} \sim r^{2} \ln N$ derivado en [26]. Sin embargo, este aumento logarítmico de $\tau_{\mathrm{MF}}$ con $N$ en $\mathrm{MF}$ es mucho más lento que el aumento no lineal $\tau \sim N^{1.64}$ obtenido en redes cuadradas. Como consecuencia, el consenso en MF es mucho más rápido que en lattices. 


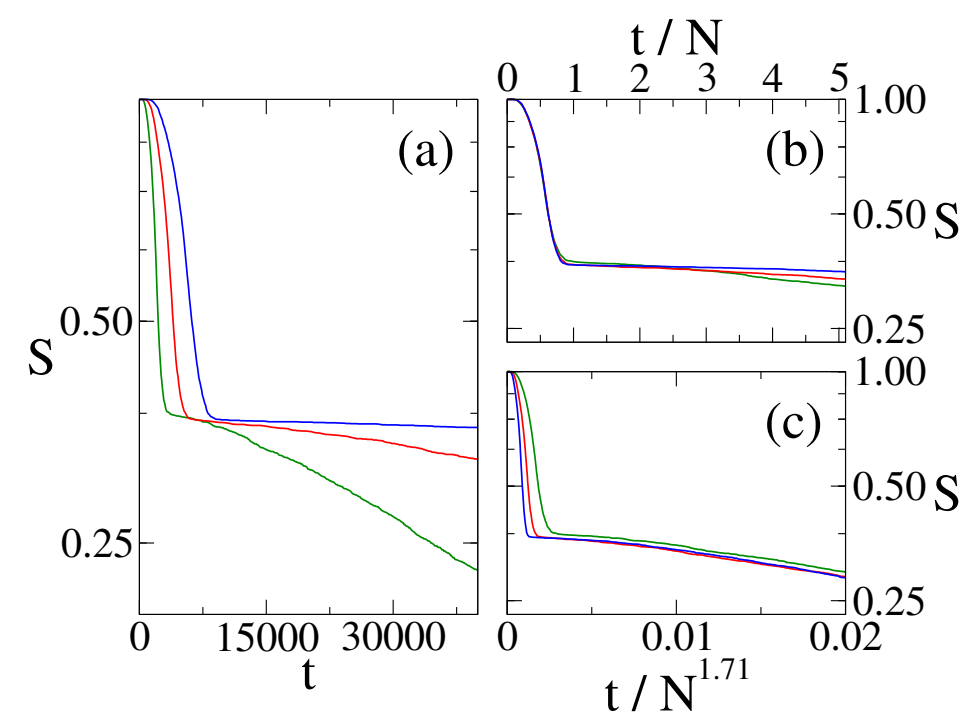

Figura 2.5: (a) Probabilidad de supervivencia $S$ en función del tiempo en escala lineal-logarítmica, para $r=1 / 3$ y tamaños del sistema $N=3600,6400$ y 10000 (de abajo hacia arriba). El rápido decaimiento inicial de $S$ describe el coarsening de los dominios, que tiene un tiempo de vida medio proporcional a $N$ [panel (b)]. La larga cola exponencial de $S$ decae con una constante de tiempo proporcional a $N^{1.71}$, asociada con la vida media de las realizaciones de tipo 2 [panel (c)].

Los largos tiempos de consenso en redes cuadradas para valores grandes de $r$ son consecuencia de los estados metaestables que caracterizan las realizaciones de tipo 2 discutidas en la sección 2.2, y que conducen al exponente no trivial de 1.64. De hecho, el valor de $\tau$ obtenido en simulaciones es la combinación de los dos tipos de realizaciones que tienen escalas de tiempo muy diferentes. Las realizaciones de tipo 1 , donde se alcanza el consenso rápido mediante la formación de dominios y las realizaciones de tipo 2 en las que se llega al consenso mediante la difusión de las dos interfases definidas por bandas. Para distinguir entre realizaciones de tipo 1 y tipo 2, seguimos el método desarrollado en [64,72 y estudiamos la distribución de los tiempos de consenso $P(t)$, donde este tiempo se calcula como $\tau=\int_{0}^{\infty} t P(t) d t$. Esto es equivalente a estudiar la probabilidad de supervivencia $S(t)$ de realizaciones individuales definida como la probabilidad de que una realización no llegue al consenso hasta un tiempo $t$, que está relacionado con $P(t)$ por la expresión $S(t)=1-\int_{0}^{t} P(t) d t$. La ventaja de calcular $S$ en lugar de $P$ es que $S$ tiene menos fluctuaciones asociadas al número finito de realizaciones. La figura 2.5 (a) muestra $S$ en función del tiempo para $r=1 / 3$ y tres tamaños de sistema. De acuerdo con los resultados en modelos relacionados 64, 72, las curvas se caracterizan por dos escalas de tiempo: una escala de tiempo breve consistente con una 


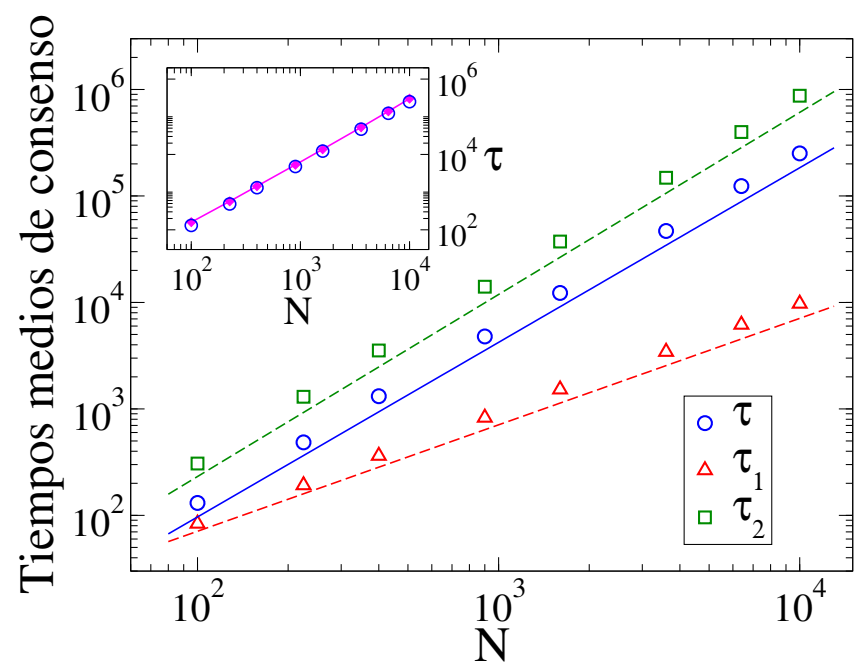

Figura 2.6: Tiempos medios de consenso $\tau, \tau_{1}$ y $\tau_{2}$ en función del tamaño del sistema $N$ en escala doble logarítmica para $r=1 / 3$. $\tau$ es el promedio sobre todas las $10^{4}$ realizaciones, mientras que $\tau_{1}$ y $\tau_{2}$ corresponden a los valores promedios sobre las realizaciones de tipo 1 y tipo 2 , respectivamente. Las líneas sólidas tienen pendientes 1.71, 1.64 y 1.0 (de arriba hacia abajo). Gráfico interno: estimación del tiempo medio de consenso como la combinación lineal $0.66 \tau_{1}+0.34 \tau_{2}$ (diamantes) comparados con $\tau$ (círculos) para ambos tipos de realizaciones.

decaimiento rápido al consenso, y una escala de tiempo mucho más larga asociada con un decaimiento exponencial asintótico (la cola). El decaimiento rápido inicial de $S$ corresponde al consenso inducido por la formación de dominios observado en las realizaciones de tipo 1, mientras que la cola exponencial describe los tiempos de consenso de las realizaciones que quedan atrapadas en un estado metaestable de banda (de tipo 2). El tiempo $t^{*}$ al que comienza la caída exponencial fue tomado como referencia para asignar un tipo a una realización dada. Realizaciones que alcanzaron el consenso antes (después) de $t^{*}$ se consideraron de tipo 1 (tipo 2). Usando este criterio, calculamos el tiempo para llegar a un consenso en cada tipo de realización.

En la Fig. 2.6 mostramos que el tiempo medio de consenso tiene una escala que va como $\tau_{1} \sim N$ en las realizaciones de tipo 1 , mientras que para las realizaciones de tipo 2 la escala es $\tau_{2} \sim N^{1.71}$. El colapso de los datos en los paneles (b) y (c) de la figura 2.5 muestra que $\tau_{1}$ puede considerarse como la escala de tiempo característica asociada al rápido decaimiento inicial de $S$, y que $\tau_{2}$ es proporcional a la constante de tiempo del decaimiento exponencial. También hemos calculado la probabilidad de que una realización llegue al estado metaestable como la fracción de 
realizaciones de tipo 2 sobre $10^{3}$ realizaciones independientes, que dieron un valor medio aproximado 0.34 en un rango de tamaños de sistema $400 \leq N \leq 10000$, con una disminución muy lenta a medida que $N$ aumenta. La estimación indirecta del tiempo medio de consenso como la combinación de los dos tipos de realizaciones puede escribirse

$$
\tau \simeq 0.66 \tau_{1}+0.34 \tau_{2}
$$

y se muestra en el gráfico interno de la Fig. 2.6 (diamantes sólidos), donde observamos la coincidencia con el valor de $\tau$ calculado realizando un promedio sobre todas las realizaciones (círculos vacíos). Por lo tanto, el escaleo aproximado $\tau \sim N^{1.64}$ observado en simulaciones puede ser explicado como el resultado de la combinación lineal de los comportamientos de ley de potencia $\tau_{1} \sim N$ y $\tau_{2} \sim N^{1.71}$. Como $\tau_{2}$ se vuelve mucho más grande que $\tau_{1}$ a medida que $N$ aumenta por un factor de 10 (100) para $N=400\left(10^{4}\right)$, esperamos que el exponente efectivo 1.64 para $\tau$ pueda aproximarse al exponente de $\tau_{2}$ cuando $N$ aumente. En la próxima sección se proporcionará una explicación más detallada para la aparición de este exponente no trivial 1.71 estudiando la dinámica de las interfases en forma de bandas.

\subsection{Dinámica de las bandas hacia el consenso}

En la sección previa 2.3 mostramos que el tiempo medio de consenso para $r=1 / 3$ escalea como $\tau \sim N^{1.64}$ con el tamaño del sistema $N$. Como se discutió anteriormente, este escaleo se debe principalmente a la existencia de estados metaestables que sobreviven durante mucho tiempo, en los que el sistema muestra una especie de patrones banda. Es importante mencionar que leyes de escala muy similares para el tiempo de consenso con el tamaño del sistema, $\tau \sim N^{\nu}$, ya se conocían dentro de la literatura, específicamente en trabajos relacionados sobre redes bidimensionales 64, 65,72. Por ejemplo, en el Modelo de la Regla de la Mayoría (MR por sus siglas en inglés) introducido en [72, los autores encontraron un valor de $\nu=1.7$, mientras que en el modelo bilingüe estudiado en [64] este exponente tiene un valor de $\nu=1.8$, y también se observó un exponente similar en el VM con grados de confianza investigado en [65], cuyo valor exacto no fue reportado. Lo que todos estos modelos tienen en común con el modelo $\mathrm{M}$ en una red bidimensional es la existencia de estados banda con una probabilidad cercana a $1 / 3$ cuando el sistema comienza desde condiciones iniciales aleatorias, y un estado de consenso final que es absorbente. A pesar de que estos modelos difieren en el número de 
estados de opinión (2 estados en el modelo MR, 3 estados en el modelo bilingüe, 4 estados en el VM con grados de confianza, y 4 o más estados en el modelo M), sus reglas microscópicas inducen una dinámica de coarsening impulsada por la tensión superficial, que puede conducir a la formación de bandas horizontales, verticales o diagonales en redes cuadradas, como se conoce que ocurre en sistemas similares a Ising [73. Por lo tanto, parece que la dinámica de bandas es el mecanismo fundamental que determina los tiempos de consenso en modelos donde se emplean estas redes cuadradas con coarsening por tensión superficial y estados de consenso absorbentes con el escaleo $\tau \sim N^{\nu}$ (con $1.64 \leq \nu \leq 1.8$ ) reportado en los trabajos mencionados anteriormente. Por lo que sabemos, todavía no hay una explicación satisfactoria del comportamiento de $\tau$ con $N$. Algunos intentos de obtener el exponente $\nu$ fueron desarrollados en [72] y [65], quienes llegaron al valor aproximado $\nu=1.5$ que está lejos del exponente obtenido a partir de simulaciones numéricas de los respectivos modelos, $\nu=1.7$ y $\nu=1.8$.

En esta sección, proponemos un enfoque que da una idea de la dinámica de bandas hacia el consenso observada en el sistema y proporciona un valor de $\nu$ que concuerda con las simulaciones. La Ec. (2.4) muestra que el tiempo medio de consenso tiene una contribución lineal $\left(\tau_{1} \sim N\right)$ que corresponde a realizaciones de corta duración (tipo 1) y un término no lineal $\left(\tau_{2} \sim N^{1.71}\right)$ correspondiente a las realizaciones de larga duración (tipo 2). Dado que $\tau_{1}$ es mucho más pequeño que $\tau_{2}$ para el rango explorado de $N$ (ver Fig. 2.6), podemos suponer que $\tau$ está principalmente determinado por las realizaciones de larga duración que caen en un estado de banda (realizaciones de tipo 2). La evolución de una realización típica de tipo 2 consiste en dos diferentes etapas, como podemos ver en la evolución de $\rho$ en la Fig. 2.3 (b). La etapa inicial se caracteriza por la dinámica de coarsening donde $\rho$ exhibe un decaimiento de ley de potencia hasta un tiempo $t \simeq 10^{4}$. Luego, el sistema cae en un estado metaestable de bandas donde $\rho$ se mantiene casi constante hasta que se llega al consenso a tiempo $t \simeq 2 \times 10^{6}$. Por lo tanto, vemos que el tiempo de consenso es controlado en gran medida por la duración de esta etapa de bandas, ya que es mucho más larga que la etapa inicial de formación de dominios (coarsening).

Para estudiar la dinámica de las bandas partimos de una condición inicial que consistía en dos franjas verticales de ancho $L / 2$ cada una, como vemos en la Fig. 2.7 (a-I). La Fig. 2.7 (a) muestra una evolución típica de las bandas en una sola realización, donde combinamos ambas opiniones de una misma orientación en un solo color para representar más claramente las interfases $(-1$ y -2 en azul, 

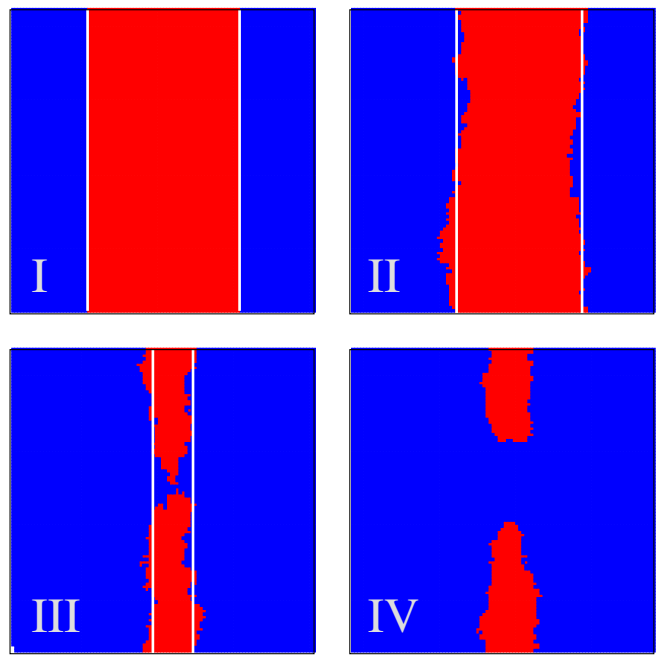

(a)

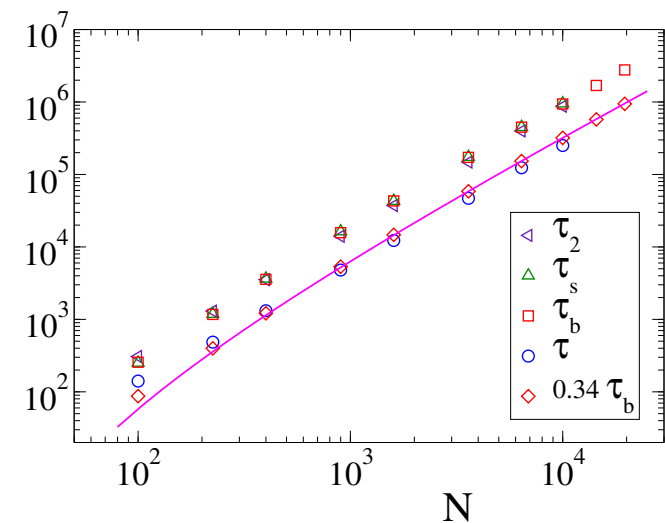

(b)

Figura 2.7: (a) Imágenes espaciales del sistema de red bidimensional $100 \times 100$ en cuatro instantes diferentes de tiempo, mostrando la evolución de las bandas de misma opinión/orientación en una realización (opiniones negativas -1 y -2 en azul y opiniones positivas 1 y 2 en rojo). Las líneas sólidas verticales denotan la posición de las interfases. (b) Comparación del tiempo medio de consenso para realizaciones de tipo 2 (bandas) que inician con condiciones aleatorias $\tau_{2}$ (triángulos hacia la izquierda), con el tiempo medio de consenso $\tau_{s}$ (triángulos hacia arriba) y el tiempo medio de ruptura de la interfase $\tau_{b}$ (cuadrados) cuya condición inicial se realiza desde una configuración de bandas mostradas en la Fig. I del panel (a). Tambien se compara el tiempo medio de consenso $\tau$ (círculos) con la estimación $0.34 \tau_{b}$ (diamantes). La línea sólida corresponde a la aproximación analítica de la Ec. (2.11).

1 y 2 en rojo). Las interfases que separan las bandas difunden libremente en la dirección perpendicular a las mismas [Fig. 2.7 (a-II)] hasta que se encuentran y se aniquilan, separándose en dos dominios [Fig. 2.7 (a-III)]. Luego, durante la última etapa, éstos dominios se reducen rápidamente [Fig. 2.7 (a-IV)] y desaparecen, para finalmente alcanzar el consenso en el sistema. Como esta última etapa es mucho más corta que la etapa difusiva, el tiempo medio de consenso a partir de un estado inicial de banda, llamado $\tau_{s}$, puede ser aproximado como el tiempo medio requerido para que las dos interfases se encuentren y se rompan, a este tiempo lo llamamos "tiempo medio de ruptura" $\tau_{b}$. En la Fig. 2.7 (b) verificamos que $\tau_{b}$ (cuadrados) es de hecho muy similar a $\tau_{s}$ (triángulos hacia arriba). También vemos que $\tau_{b}$ es 
similar al tiempo medio de consenso $\tau_{2}$ de las realizaciones de tipo 2 comenzando desde una condición inicial aleatoria (triángulos hacia la izquierda), como sugerimos anteriormente.

Usando la Ec. (2.4) encontramos que $\tau$ puede ser escrito aproximadamente como

$$
\tau \simeq 0.34 \tau_{b}
$$

representado por diamantes en la Fig. 2.7(b).

Basados en este resultado, en la subsección 2.4.1, derivamos una aproximación analítica para la dependencia de $\tau_{b}$ con $L$ usando las propiedades de difusión de las interfases, y en la subsección 2.4.2 mejoramos esta aproximación incorporando las propiedades de rugosidad de las mismas.

\subsubsection{Estimación de $\tau_{b}$ considerando dos partículas difusi- vas}

Para estudiar con más detalle la dinámica de las interfases, empezaremos definiendo la posición $x_{i}(t)$ de la interfase $i(i=1,2)$ para un dado tiempo $t$ como el valor medio de todas las posiciones de la misma $x_{i, y}(t)$ a cierta altura $y$ [ver Fig. 2.8(a)]

$$
x_{i}(t)=\frac{1}{L} \sum_{y=1}^{L} x_{i, y}(t) .
$$

Entonces, podemos interpretar $x_{1}$ y $x_{2}$ como las posiciones respectivas de dos partículas puntuales independientes que difunden en un intervalo $[1, L]$ con condiciones de contorno periódicas, y que se aniquilan cuando se encuentran. Esta equivalencia fue propuesta por Chen y Redner en el modelo MR [72], y también fue usada más tarde por Volovik y Redner en el VM con grados de confianza 65]. Verificamos que la partícula 1 (y también la partícula 2) se mueve de forma difusiva midiendo la evolución temporal de la varianza de $x_{1}, \sigma^{2}(t)=\left\langle x_{1}^{2}\right\rangle(t)-\left\langle x_{1}\right\rangle^{2}(t)$, donde los promedios se hicieron sobre $10^{4}$ realizaciones independientes. Encontramos que $\sigma^{2}(t)$ aumenta linealmente con el tiempo para varios tamaños de $L$ y que el coeficiente de difusión $D_{L}$, calculado a partir de la relación $\sigma^{2}(t)=2 D_{L} t$ de un proceso difusivo, decae como $1 / L$ (no mostrado). De hecho, observamos que todas las curvas colapsan cuando el eje $y$ es reescalado por $L$, obteniendo la relación aproximada

$$
D_{L} \simeq \frac{d}{L}
$$




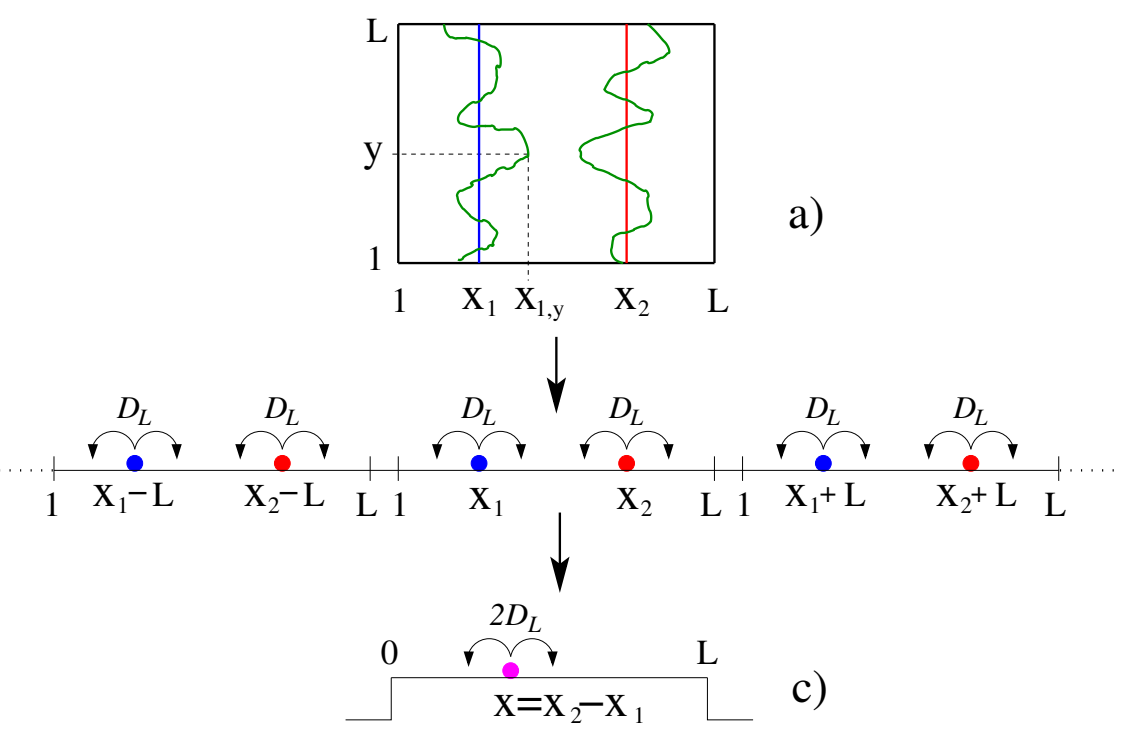

b)

Figura 2.8: Ilustración del mapeo de la dinámica de la interfase partiendo de la condición de bandas para el problema de las dos partículas puntuales difusivas en el intervalo $[1, L]$ con condiciones periódicas de contorno. a) Las líneas verticales indican las posiciones $x_{1}$ y $x_{2}$ de las interfases, denotadas por círculos (partículas) en el panel b). b) El sistema es replicado en todo el espacio 1D, donde las partículas difunden libremente sin restricciones en los bordes. c) Partícula puntual equivalente con posición $x=x_{2}-x_{1}$ que difunde en el intervalo $[0, L]$ con límites absorbentes en los extremos. El coeficiente de difusión $2 D_{L}$ es el doble que el de las partículas en el panel a).

con $d=0.04$. Una estimación de esta relación de escaleo se desarrolló en 65,72 suponiendo que cada punto en la interfase $x_{i, y}$ se comporta como un caminante aleatorio independiente 74,75 que salta un sitio a la derecha o a la izquierda con igual probabilidad, entonces, $\sqrt{D_{L}}$ debe ser proporcional al desplazamiento medio de la posición de la interfase $x_{1}$ en un intervalo de tiempo $\Delta t=1$, que escalea con el número de caminantes $L$ como $\sqrt{L} / L=L^{-1 / 2}$, por lo tanto $D_{L} \sim 1 / L$.

Ahora podemos aproximar el tiempo medio de ruptura de la interfase $\tau_{b}$ como el tiempo medio en que las partículas tardan en encontrarse en el intervalo $[1, L]$, cuando sus posiciones iniciales están a una distancia $L / 2$. Dada la naturaleza periódica de los bordes en este intervalo, resulta útil considerar un sistema equivalente que se obtiene al replicar el intervalo y las partículas en el espacio unidimensional $(1 D)$ [ver Fig. 2.8 (b)], donde estas puedan difundir libremente en todo el espacio de $1 D$ sin restricciones de borde. En este sistema replicado, la partícula 1 se mueve siempre entre la partícula 2-izquierda y la partícula $2\left(x_{2}-L \leq x_{1} \leq x_{2}\right)$ hasta que 
se aniquile con una de estas dos partículas $\left(x_{1}=x_{2}-L\right.$ ó $\left.x_{1}=x_{2}\right)$. Por lo tanto, la diferencia $x \equiv x_{2}-x_{1}$ se puede ver como la posición de una partícula equivalente que difunde en el intervalo $[0, L]$ con condiciones de contorno absorbentes en $x=0$ y $x=L$ [ver Fig. 2.8 (c)]. Entonces, el problema se reduce al escape de una partícula con difusión $2 D_{L}$ (dos veces la de las partículas 1 y 2 ) en el intervalo $[0, L]$ a partir de una posición $x=L / 2$, cuya expresión exacta para el tiempo medio de salida es $L^{2} / 16 D_{L}$ (ver, por ejemplo [25]). Después de reemplazar la expresión de la Ec. 2.7) por $D_{L}$ obtenemos

$$
\tau_{b}^{\mathrm{I}}=\frac{L^{3}}{16 d}
$$

donde el superíndice I en $\tau_{b}^{\mathrm{I}}$ es usado para indicar una aproximación de primer orden (vea la siguiente subsección para aproximaciones de orden superior). En la Fig. 2.9 comparamos la expresión de la Ec. 2.8 para $\tau_{b}^{\mathrm{I}}$ (línea punteada) con el valor de $\tau_{b}$ obtenido en simulaciones numéricas (círculos). Aunque vemos que $\tau_{b}^{\mathrm{I}}$ es una aproximación razonable para $\tau_{b}$, se sobreestima este tiempo para todos los valores simulados de $L$. Sin embargo, mostraremos que $\tau_{b}^{\mathrm{I}}$ se acerca asintóticamente a $\tau_{b}$ en el límite $L \rightarrow \infty$. Esta observación ya fue reportada en 65,72 junto con el escaleo aproximado $\tau \sim L^{3}=N^{3 / 2}$.

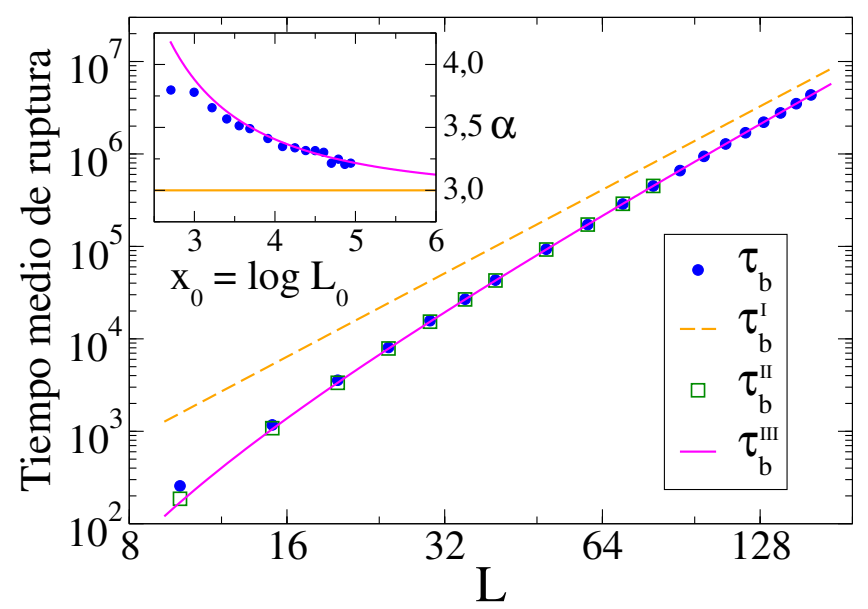

Figura 2.9: Tiempo medio de ruptura de la interfase vs lado de la red $L$ mostrado en escala log-log con una condición inicial de bandas (Fig. 2.7)(a-I)). Se comparan los resultados de simulación ( $\tau_{b}$, círculos llenos) con las siguientes expresiones aproximadas: $\tau_{b}^{\mathrm{I}}$ (línea punteada) Ec. (2.8), $\tau_{b}^{\mathrm{II}}$ (cuadrados vacíos) Ec. (2.9) y $\tau_{b}^{\mathrm{III}}$ (línea sólida) Ec. 2.10). Gráfico interno: Los círculos corresponden a la pendiente de la curva $\tau_{b}$ vs $L$ en escala doble logarítmica, calculada con los datos de la Figura principal, mientras que la línea sólida es la aproximación analítica Ec. (2.14). 


\subsubsection{Estimación de $\tau_{b}$ considerando dos barras difusivas}

El tiempo medio de ruptura $\tau_{b}^{\mathrm{I}}$ se puede considerar como una primera aproximación para $\tau_{b}$, donde se supone que las interfases de las bandas se rompen cuando sus posiciones sean exactamente iguales $\left(x_{1}=x_{2}\right)$. Sin embargo, esta aproximación no toma en cuenta la rugosidad de cada interfase, que, como veremos, juega un papel importante en la dinámica de ruptura. En una aproximación más refinada que tenga en cuenta el ancho de las interfases se debe considerar que, en una realización dada, las interfases se rompen cuando están ubicadas a alguna distancia $\Delta x^{b}=\left|x_{2}^{b}-x_{1}^{b}\right|>0$ [ver Fig. 2.10 (a)], donde $x_{1}^{b}$ y $x_{2}^{b}$ son las respectivas posiciones de las interfases en el momento de la ruptura. La idea detrás de este argumento es que la ruptura ocurre cuando las interfases se tocan por primera vez en algún punto y que depende de la rugosidad específica de las mismas en ese momento, como vemos en la Fig. 2.10 (a). Por lo tanto, cada interfase se puede describir mejor mediante una partícula difusiva con forma de barra rígida de longitud $\Delta x^{b}$ que representa el ancho de las interfases en el momento de la ruptura [ver Fig. 2.10 (b)]. Estas dos barras difunden hasta que colisionan y se aniquilan en una de las dos formas posibles que se muestran en los paneles b) y c) de la figura 2.10. En el sistema replicado, el centro de la barra 1 se mueve entre las posiciones $x_{1}=x_{2}-\Delta x^{b}$ [panel b)] y $x_{1}=x_{2}-L+\Delta x^{b}$ [panel c)], y por lo tanto, la diferencia $x=x_{2}-x_{1}$ entre los centros de las barras describe la posición de una partícula puntual que se mueve en el intervalo $\left[\Delta x^{b}, L-\Delta x^{b}\right]$ de longitud reducida $L-2 \Delta x^{b}$ [paneles (d) y (e)].

Si tomamos el valor promedio de $\Delta x^{b}$ sobre muchas realizaciones de la dinámica, $\left\langle\Delta x^{b}\right\rangle$, como la distancia efectiva entre las interfases cuando se tocan por primera vez, el problema se puede reducir al escape de una partícula en un intervalo de "longitud efectiva" $\bar{L}=L-2\left\langle\Delta x^{b}\right\rangle$. Entonces, el tiempo medio de escape será $\bar{L}^{2} / 16 D_{L}$ o, reemplazando la expresión anterior para $\bar{L}$ y la Ec. 2.7 para $D_{L}$ nos queda

$$
\tau_{b}^{\mathrm{II}}=\frac{\left(1-2\left\langle\Delta x^{b}\right\rangle L^{-1}\right)^{2} L^{3}}{16 d} .
$$

La Ec. 2.9) representa una segunda aproximación que incorpora la distancia promedio entre las interfases cuando se encuentran. Para probarla (Ec. (2.9p) ejecutamos simulaciones y medimos la distancia promedio de la interfase $\left\langle\Delta x^{b}\right\rangle$ para varios valores de $L$ [cuadrados en la Fig. 2.11(a)]. El momento en el que se rompe la interfase para una realización determinada se tomó como el tiempo para el cual 

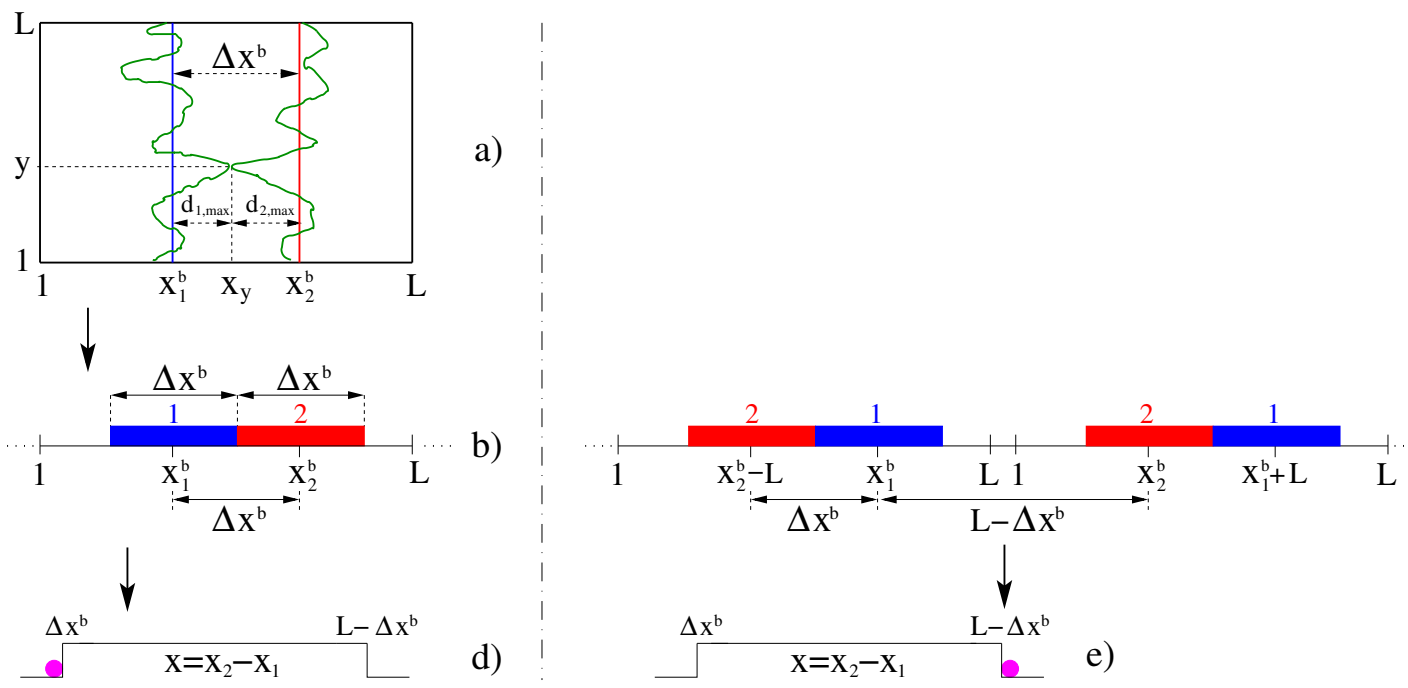

c)

Figura 2.10: a) Las interfases entre las bandas se rompen cuando están a una distancia $\Delta x^{b}$ y se tocan en un punto $y$ por primera vez. b) y c) Las interfases se mapean a barras replicadas en el espacio $1 D$ que se aniquilan cuando chocan. d) y e) Partícula puntual equivalente con posición $x=x_{2}-x_{1}$ que difunde en el intervalo $\left[\Delta x^{b}, L-\Delta x^{b}\right]$ con límites absorbentes en los extremos.

todos los sitios de al menos una fila de la red tienen estado 2 ó -2 por primera vez. Los cuadrados vacíos en la Fig. 2.9 representan la estimación $\tau_{b}^{\text {II }}$ de $\tau_{b}$ obtenida al insertar el valor numérico de $\left\langle\Delta x^{b}\right\rangle$ en la Ec. (2.9), lo cual coincide bastante bien con los resultados de la simulación (círculos llenos) para $L \geq 15$. Esto muestra que la rugosidad de la interfase juega un papel muy importante en la dinámica de ruptura, produciendo grandes desviaciones de $\tau_{b}$ en la ley de escala $L^{3}$ (línea punteada en Fig. 2.9 cuando $L$ disminuye. Estas desviaciones, que se vuelven más visibles para valores pequeños de $L$, son capturadas bastante bien por el prefactor $\left(1-2\left\langle\Delta x^{b}\right\rangle / L\right)^{2}$ de $\tau_{b}^{\mathrm{II}}$ en la Ec. 2.9. Vemos en la Fig. 2.11 (a) que $\left\langle\Delta x^{b}\right\rangle$ crece con $L$ como $L^{0.525}$ (línea continua), y por lo tanto la proporción $\left\langle\Delta x^{b}\right\rangle / L$ desaparece a medida que $L$ aumenta, lo que lleva a la expresión de la Ec. 2.8 para $\tau_{b}^{\mathrm{I}}$, confirmando la hipótesis de que la Ec. 2.8 es correcta en el límite $L \rightarrow \infty$. Como se muestra en el apéndice $\mathrm{A}$, el exponente 0.525 está relacionado con el exponente de rugosidad $\alpha \simeq 0.5$ asociado al valor de saturación del ancho de las interfases. Una conclusión interesante de la Ec. 2.9 es que una ley de potencia pura $\tau_{b}^{\mathrm{II}} \sim L^{2 \nu}=N^{\nu}$ nunca se obtiene para un valor finito de $L$. En cambio, el factor de corrección $\left(1-2\left\langle\Delta x^{b}\right\rangle / L\right)^{2}$ introduce una curvatura descendente en la curva $\tau_{b}^{\mathrm{II}}$ vs $L$ en una escala doble logarítmica, que disminuye con $L$ y se vuelve muy pequeña para $L \gtrsim 40$ (ver Fig. 2.9). Como resultado, los datos pueden ser bien ajustados 
por una función de ley de potencia de $N$ con un exponente efectivo $\nu>1.5$, como los que se muestran en la Fig. 2.6 para $\tau$ y $\tau_{2}$.
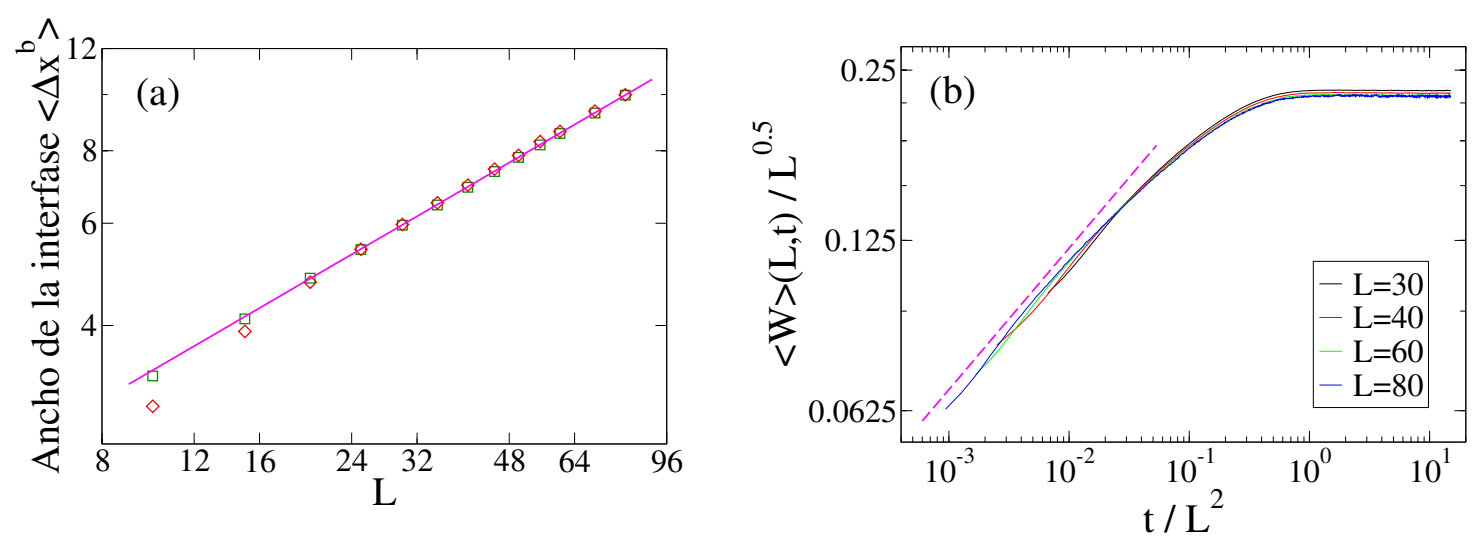

Figura 2.11: a) Distancia promedio entre interfases cuando éstas se rompen $\left\langle\Delta x^{b}\right\rangle$ (cuadrados) y desviación máxima promedio de la interfase $2\left\langle d_{\max }\right\rangle$ (diamantes) como función de $L$. La línea sólida representa el mejor ajuste de ley de potencia $L^{0.525}$ para $\left\langle\Delta x^{b}\right\rangle$. (b) Crecimiento del ancho de la interfase promedio $\langle W\rangle$ con el tiempo. El ancho y el tiempo son reescalados por $L^{0.5}$ y $L^{2}$, respectivamente, para obtener el colapso de la data para los valores de $L$ indicados en la leyenda. La línea punteada indica el crecimiento inicial tipo ley de potencia $t^{0.25}$.

Al sustituir la aproximación de ley de potencia $\left\langle\Delta x^{b}\right\rangle \simeq L^{0.525}$ en la Ec. 2.9 obtenemos la siguiente expresión aproximada para el tiempo medio de ruptura:

$$
\tau_{b}^{\mathrm{III}}=\frac{\left(1-2 L^{-0.475}\right)^{2} L^{3}}{16 d}
$$

Como podemos ver en la Fig. 2.9, la Ec. 2.10 representada por la línea sólida, ajusta muy bien con los datos numéricos (círculos llenos) para $L \gtrsim 15$. Finalmente, usando la Ec. (2.5) llegamos la expresión

$$
\tau \simeq \frac{0.34\left(1-2 N^{-0.2375}\right)^{2} N^{1.5}}{16 d}
$$

para el tiempo medio de consenso. La Ec. 2.11) está representada por la línea sólida en la Fig. 2.7(b). Vemos que, aunque hay algunas discrepancias con los resultados numéricos (círculos), la Ec. 2.11) captura bastante bien el comportamiento de $\tau$ con el tamaño del sistema $N$ para casi la totalidad del rango de valores.

Ahora podemos explorar la forma funcional aproximada de $\tau_{b}$ dada por la Ec. 2.10 para analizar la escala de $\tau_{b}$ para un amplio rango de $L$. El factor $L^{-0.475}$ 
introduce una curvatura hacia abajo en $\tau_{b}^{\mathrm{III}}$-cuando se grafica en escala log-log-que desaparece cuando $L$ aumenta. Por lo tanto, podemos aproximarnos a la forma de $\tau_{b}^{\mathrm{III}}$ alrededor de un valor dado $L_{0}$ como una ley de potencias de $L$ (ver el apéndice B para detalles de cálculo)

$$
\tau_{b}^{\mathrm{III}}\left(L, L_{0}\right) \simeq A\left(L_{0}\right) L^{\alpha\left(L_{0}\right)}
$$

donde

$$
\begin{aligned}
& A\left(L_{0}\right)=\frac{1}{16 d}\left(1-2 L_{0}^{-0.475}\right)^{2} L_{0}^{\frac{1.9}{\left(2-L_{0}^{0.475}\right)}} \quad \mathrm{y} \\
& \alpha\left(L_{0}\right)=3+\frac{1.9}{\left(L_{0}^{0.475}-2\right)}
\end{aligned}
$$

Podemos verificar que en el límite termodinámico $L_{0} \rightarrow \infty$ el exponente $\alpha\left(L_{0}\right)$ se aproxima al valor 3.0, como se sugirió anteriormente, mientras que $A\left(L_{0}\right)$ se acerca a $1 /(16 d)$, recuperando la aproximación $\tau_{b}^{\mathrm{I}} \simeq L^{3} /(16 d)$ de la Ec. (2.8). El exponente $\alpha\left(L_{0}\right)$ de la Ec. 2.14), que mide la pendiente de la curva $\log \left[\tau_{b}^{\mathrm{III}}(L)\right]$ vs $\log (L)$ en algún punto $\log \left(L_{0}\right)$, es trazado por una línea continua en la Fig. interna de la Fig. (2.9) y comparado con el valor numérico (círculos llenos) obtenido calculando la pendiente local de los puntos de $\tau_{b}$ en la Fig. principal. Podemos ver que la pendiente disminuye muy lentamente con $L_{0}$, y por lo tanto para los valores de $L$ medidos en las simulaciones $\alpha$ permanece casi constante y puede ser aproximada por una ley de potencia pura. Entonces podemos usar la Ec. 2.14 para aproximar el tiempo medio de ruptura como $\tau_{b} \sim L^{\alpha}=N^{\alpha / 2}$ en el rango de tamaños de sistema utilizados en las simulaciones, y comparar $\alpha$ con los exponentes numéricos obtenidos de la Fig. 2.6 por ajuste de los datos numéricos con una ley de potencia. Por ejemplo, la pendiente en $N=2000(L \simeq 45)$ de la Ec. (2.14) es $\alpha / 2 \simeq 1.73$, lo que coincide bastante bien con la pendiente numérica 1.71 para $\tau_{2}$ en el rango $4 \times 10^{2} \leq N \leq 10^{4}$. El valor teórico $\alpha / 2$ también es una aproximación del exponente numérico 1.64 obtenido de los datos de $\tau$ vs $N$ (solo $5.5 \%$ menos), aunque esperamos que esta aproximación mejore para valores mayores de $N$. Finalmente, también notamos que se vuelve muy difícil alcanzar una pendiente cercana a 1.5 en simulaciones del modelo, debido a la disminución muy lenta de $\alpha$ con $L_{0}$. Por ejemplo, para lograr una pendiente menor a 1.545 (menos de $3 \%$ de diferencia con 1.5), la Ec. 2.14 predice que necesitaríamos ejecutar simulaciones en sistemas con valores de $L \gtrsim 750$, cuyos tiempos de consenso son del orden de $\tau \sim 10^{9}$ (Ec. 2.10), que es casi imposible de lograr en tiempos de cálculo razonables. 


\subsection{Discusiones finales}

En este capítulo, estudiamos un modelo basado en agentes que interactúan en una red bidimensional que explora la competencia entre la persuasión y el compromiso en la formación de opiniones. Descubrimos que las interacciones a primeros vecinos entre los agentes inducen a una dinámica de coarsening muy interesante que desempeña un papel fundamental en la evolución del sistema y la aproximación hacia el consenso. Las propiedades de coarsening dependen fuertemente de la frecuencia relativa entre los eventos de persuasión y compromiso, medida por la relación $r=p / q$ entre las probabilidades de interacción de persuasión y compromiso. Cuando el proceso de compromiso domina sobre el proceso de persuasión, la dinámica es similar a la del VM durante un corto tiempo transitorio inicial, en el que los dominios están formados por agentes moderados y el coarsening no tiene tensión superficial. Esto está asociado a un estado de opinión centralizado donde la mayoría de los agentes adoptan valores de opinión moderados. El crecimiento del dominio finalmente conduce a un estado donde todos los agentes tienen la misma orientación de opinión (positiva o negativa). Luego, los agentes moderados comienzan a convertirse en extremistas y el sistema muestra un comportamiento exponencial lento hacia el consenso de una opinión extrema, que se logra en un tiempo que escalea como $r^{-1} \ln N$ con el tamaño de la población $N$. En el escenario opuesto, donde la persuasión domina sobre el compromiso, el coarsening es impulsado por la tensión superficial y los agentes moderados se ubican en la interfase entre los dominios formados por los extremistas. Esto corresponde a un estado de opinión polarizado en el que la población se divide en dos grupos que adoptan opiniones extremas y opuestas (positiva y negativa). La aproximación final al consenso puede ser muy larga si el sistema cae en una configuración metaestable de bandas, donde las dos interfases que definen una banda difunden hasta que se encuentran y aniquilan. El tiempo medio de consenso de este tipo de realizaciones escalea como $N^{1,71}$. Cuando el promedio se realiza sobre todas las realizaciones, que incluyen realizaciones de corta duración con un tiempo que escalea como $N$, el tiempo medio de consenso general escalea como $\tau \sim N^{\nu}$, con $\nu=1,64$.

Se propuso una aproximación para describir el consenso de las configuraciones de bandas al mapear esta dinámica a un problema de dos barras que difunden libremente en $1 D$ y se aniquilan cuando colisionan por primera vez. Este método tiene en cuenta el ancho de las interfases-banda, que se vuelve relevante cuando éstas 
se encuentran y se rompen. Una estimación analítica del tiempo medio de ruptura usando resultados conocidos en problemas de primer pasaje permitió obtener la expresión aproximada (Ec. 2.10) para la vida media de las bandas, lo cual coincide con los resultados de las simulaciones del modelo. Además, la Ec. 2.11 para el tiempo medio de consenso muestra que el escaleo $\tau \sim N^{\nu}$ es una aproximación obtenida ajustando con una ley de potencia los datos numéricos sobre un rango finito de $N$, ya que el exponente efectivo $\nu$ alrededor de un $N$ dado disminuye y se acerca al valor 1.5 en el límite termodinámico $(N \rightarrow \infty)$. Estos resultados muestran que las desviaciones analíticas del exponente de escala $\nu=1.5$, obtenidas al suponer que las interfases se comportan como partículas puntuales, se deben a la rugosidad de las mismas.

Además, las interacciones en la topología espacial de $2 D$ tiene un gran impacto en el comportamiento del modelo M respecto del caso de MF. La bipolarización de opinión es mucho más estable en redes bidimensionales que en MF, debido a la existencia de estados metaestables de larga duración con un patrón espacial de opiniones que consiste en dos bandas compuestas por ambos tipos de extremistas. Esta dinámica conduce a tiempos de consenso en redes cuadradas bidimensionales que son mucho más largos que los obtenidos en una configuración MF. Otra observación es que la bipolarización se encuentra para $p>q$ en MF, mientras que en redes cuadradas bidimensionales se encuentra para valores de persuasión mucho más bajos, aproximadamente por $p>q / 3$. Por lo tanto, un pequeño nivel de homofilia es suficiente para inducir bipolarización en una población que interactúa en éstas redes. En consecuencia, la topología reticular parece intensificar el efecto de la homofilia y la PAT en la aparición de la bipolarización. Este resultado se asemeja al obtenido en el modelo de Scheling para la segregación racial [76], donde una pequeña preferencia por tener vecinos de la misma raza en una red cuadrada puede inducir una gran segregación espacial de la población en dominios de la misma raza. 


\section{Capítulo 3}

\section{Propagación de Opiniones y Enferme- dades en Redes Múltiples Interactuantes}

La formación de opiniones y la propagación de enfermedades en una población de individuos son los procesos más estudiados en redes complejas en los últimos años [1,77. El comportamiento de cada uno de estos dos procesos ha sido explorado de manera independiente, y sus propiedades de propagación en diversas topologías ya están bien definidas (ver Reviews [1] y [78] en formación de opiniones y propagación de enfermedades, respectivamente). Sin embargo, se ha prestado poca atención a un posible caso donde la dinámica de las opiniones interactúa con la de la propagación de la enfermedad.

De hecho, estas dos dinámicas no están aisladas en sociedades reales, más bien se relacionan y se afectan mutuamente, ya que ambas ocurren al mismo tiempo y en la misma población: un individuo puede transmitir una enfermedad a un colega mientras tiene una conversación e intercambian ideas u opiniones sobre un tema determinado. Entonces, las siguientes preguntas surgen: ¿Cuál es el impacto de la dinámica de opiniones sobre la extensión y prevalencia de una epidemia?, ¿Facilita la propagación de una enfermedad el dominio de una opinión o, por el contrario, impide el consenso de opiniones?

En un intento por explorar estas preguntas, en este capítulo estudiamos cómo los procesos de formación de opiniones y propagación de enfermedades se afectan entre sí, usando dos modelos simples como una aproximación de cada proceso: el modelo de votante (VM), descrito en la sección 1.3.2 y el proceso de contacto (CP) descrito en la sección 1.4.1 del capítulo 1.

El VM fue presentado originalmente como el sistema más simple de partículas interactuantes que pueden resolverse con exactitud en cualquier dimensión $4,31$. 
79], y es uno de los modelos más estudiados para el consenso de opinión. En este modelo, las personas pueden tomar una de las dos posiciones u opiniones posibles en un tema dado, y se les permite actualizarlas adoptando la opinión de un vecino elegido al azar.

El CP, por su parte, ha sido ampliamente estudiado para explorar la propagación de una infección en un sistema de agentes interactuantes [80], donde los agentes infectados pueden transmitir la infección a vecinos susceptibles en una red bidimensional [29] o en una red compleja [81], y también pueden recuperarse a un ritmo determinado. El CP exhibe una transición continua desde una fase sana a una fase endémica cuando la tasa de infección excede un valor umbral.

Para modelar la interacción entre estas dos dinámicas implementamos una red compleja multicapa 22,82,83 que consiste en un conjunto de redes complejas interrelacionadas entre sí, que permite estudiar sistemas compuestos por muchos procesos interdependientes.

En nuestro sistema, la dinámica de opinión tiene lugar en una red de relaciones sociales -formada por personas que se influyen entre sí en un tema social-, mientras que la enfermedad se propaga en una red de contactos físicos -formado por personas que tienen contactos cara a cara diariamente-- Todos los individuos se encuentran en ambas redes, pero el patrón de conexiones entre ellos puede ser diferente en cada capa. La superposición de conexiones se toma como una medida del acoplamiento entre las dos redes.

El sistema de red bicapa descrito anteriormente puede representar un escenario simple donde la red social respalda un proceso que involucra presión grupal, como la adopción de nuevos comportamientos u opiniones, mientras que la red de contacto apoya la propagación de una infección viral contagiosa como la gripe, que es transmitida por proximidad o contacto directo entre individuos. Las diferentes conexiones entre individuos pueden corresponder a diferentes tipos de relaciones entre ellos. Por ejemplo, dos amigos cercanos pueden tener un vínculo social y de contacto, ya que se pueden ver en el trabajo todos los días y también intercambiar ideas sobre un tema político. También puede suceder que los individuos están conectados por un solo tipo de vínculo, por ejemplo, dos colegas que tienen una relación de contacto o de proximidad porque trabajan en el mismo lugar pero nunca hablan de política; o dos amigos que nunca se encuentran, pero discuten ideas 
políticas por medios electrónicos (teléfono, Facebook, Twitter, correo electrónico, entre otros.).

Algunos trabajos relacionados en redes multicapas $48,49,84,88$ también han explorado la interrelación entre dos procesos de difusión de información. Por ejemplo, en [48, 49] los autores analizaron cómo la toma de conciencia sobre una enfermedad, a través de la información que se conozca sobre la misma, afecta la propagación de la epidemia en una red múltiple, mediante el uso de los modelos UAU (Informado - Desinformado - Informado) y SIS (Susceptible - Infectado - Susceptible), en cada capa respectivamente. La interacción entre la formación de opinión y los procesos de toma de decisiones se estudió en [84] utilizando dos redes interconectadas. Otro trabajo consideró dos partidos políticos (dos redes que interactúan) que compiten por los votos en una elección política [85. En un artículo reciente 86, los autores estudiaron la dinámica del modelo del votante en redes bicapa con conexiones "coevolutivas", mientras que en [87] los mismos autores exploraron si es apropiado reducir la dinámica del modelo de votante de una red múltiple de dos capas a una sola capa. Un trabajo reciente [89] considera una dinámica de umbral compleja que compite con una dinámica simple Susceptible-Infectado-Susceptible en dos redes interconectadas. Todos los trabajos enumerados anteriormente exploran la interacción entre dos procesos sociales o dos procesos epidemiológicos que son similares. Sin embargo, faltan estudios específicos sobre la interacción entre la opinión y la dinámica de la enfermedad en este tipo de topologías.

\subsection{El Modelo}

Consideramos un sistema bicapa compuesto por una capa de contacto y una capa social de grado medio $\langle k\rangle=\mu$ y $N$ nodos cada una. Estas dos capas están interconectadas a través de sus nodos, que son los mismos en ambas redes, mientras que los enlaces que los conectan pueden no ser necesariamente los mismos. Es decir, ambas capas tienen el mismo número de nodos $N$ y enlaces $\mu N / 2$, pero la configuración de las conexiones puede ser diferente en cada capa. La superposición de enlaces se mide por la fracción $q(0 \leq q \leq 1)$ de enlaces compartidos en ambas redes. En nuestro modelo, los valores extremos $q=0$ y $q=1$ corresponden a redes totalmente desacopladas y totalmente acopladas, respectivamente. Para construir esta topología particular, comenzamos conectando los mismos pares de nodos al azar en ambas redes hasta que el número de enlaces alcance el valor de superposición 
$q \mu N / 2$. Luego, el resto de los enlaces $(1-q) \mu N / 2$ se colocan aleatoriamente entre los nodos de cada red por separado, asegurándonos de que el par de nodos elegido en una red ya no esté conectado en la otra red.

Los enlaces sociales en este sistema conectan a las personas que se influyen mutuamente en un tema determinado, mientras que la infección se transmite a través de enlaces de contacto. En la Fig. 3.1 ilustramos el sistema bicapa compuesto por una red social y una red de contacto (capas superior y media), y su representación como una sola capa con dos tipos de enlaces (capa inferior). Observamos en la Fig. 3.1 que los nodos $i$ y $j$ están conectados tanto por un enlace social como por uno de contacto, representando a individuos que tienen una conversación diaria cara a cara, donde intercambian opiniones y también uno de ellos puede infectar a su compañero. Los nodos $j$ y $k$ solo están conectados por un enlace social: no tienen contactos cara a cara pero aún intercambian ideas electrónicamente o por teléfono. Los nodos $i$ y $k$ solo están conectados por un enlace de contacto: tienen contactos personales o de proximidad, pero no discuten ni intercambian opiniones sobre algún problema.

Para imitar la propagación de la opinión y la enfermedad, utilizamos el Modelo de Votante (VM) y el Proceso de Contacto (CP) en cada capa, respectivamente. Cada nodo está dotado de un estado de opinión $\mathcal{O}$ que puede tomar dos valores posibles $\mathcal{O}=+,-$ (vea la capa superior de la Fig. 3.1), y un estado de enfermedad $\mathcal{D}=0,1$ que representa los estados susceptible e infectado de un individuo, respectivamente (capa media de la Fig. 3.1). Estas dos dinámicas se acoplan a través de los estados de opinión y enfermedad de los nodos, que se afectan mutuamente al reducir el flujo de información entre vecinos, como describimos a continuación con un ejemplo simple.

Consideremos una situación en la que dos personas tienen un contacto físico y social diario porque se ven en el trabajo y hablan de política. Por un lado, suponemos que cada persona está menos influenciada cuando su compañero está enfermo, ya que normalmente éste/a se queda en casa o en el hospital, reduciendo el contacto físico entre ellos. Esto hace que las relaciones sociales (y el intercambio de opiniones) sean menos probables cuando al menos uno de los dos individuos está enfermo. Por lo tanto, consideramos que tiene lugar una relación social con una probabilidad 1.0 si ambos vecinos sociales están sanos, y con una probabilidad reducida $p_{o} \leq 1.0$ cuando uno o ambos están enfermos. En caso de que tengan 


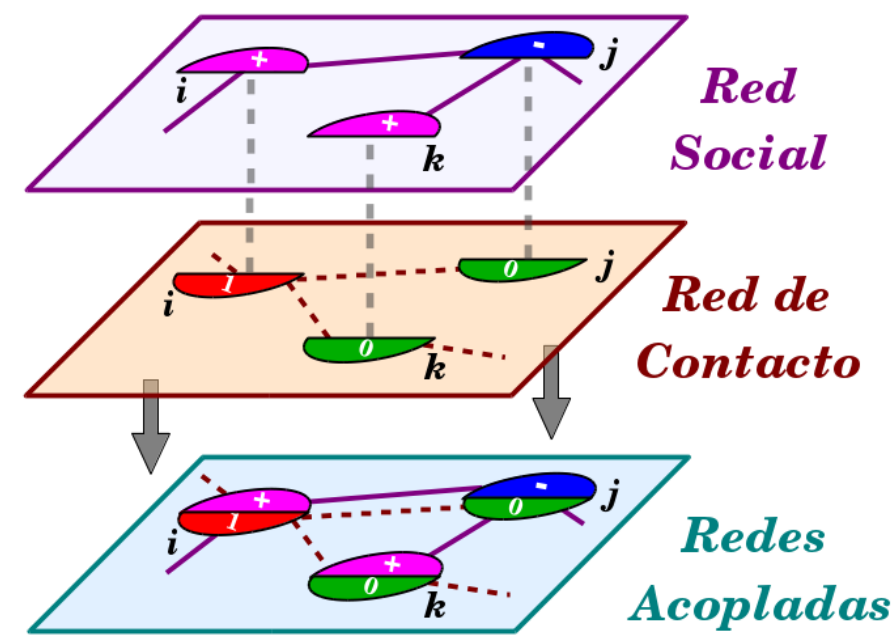

Figura 3.1: Diagrama esquemático que muestra una pequeña parte de una red múltiple de dos capas. La capa superior representa una red social donde se lleva a cabo la propagación de opiniones, mientras que la capa intermedia describe una red de contactos físicos en los que se propaga una enfermedad. La capa inferior es el colapso de ambas capas, mostrando nodos conectados por enlaces sociales (líneas continuas) y enlaces de contacto (líneas punteadas). Los estados de los nodos son Susceptible (0) e Infectado (1) en la red de contacto, y siguen la dinámica del Proceso de Contacto (CP), mientras que los estados $+\mathrm{y}-$ en la red social se actualizan de acuerdo con la dinámica del Modelo del Votante (VM).

un contacto social pero no un contacto físico (no se ven sino que discuten ideas por medios electrónicos), se supone que el estado de la enfermedad no afecta la probabilidad de interacciones sociales entre ellos. Por lo tanto, la probabilidad de interacción social no se reduce por la enfermedad y, por simplicidad, se establece en 1.0 como en el caso de los vecinos sanos.

Por otro lado, consideramos que los contactos físicos (y por lo tanto las infecciones) entre dos vecinos sociales y de contacto tienen más probabilidades de ocurrir cuando comparten la misma opinión. Esto es una consecuencia de un mecanismo sociológico llamado Homofilia [56, 90, 91], es decir, la tendencia de los individuos a interactuar con otros similares. Los efectos de la homofilia en la propagación de los atributos culturales en una sociedad fueron estudiados por Robert Alxerod usando un modelo basado en agentes [90], en el que la probabilidad de que dos agentes vecinos interactúen es proporcional a su similitud cultural (el número de atributos compartidos). Siguiendo esta idea, asumimos que la probabilidad de contacto 
entre vecinos que tienen la misma opinión es mayor que cuando tienen opiniones diferentes. Por lo tanto, establecemos en 1.0 la probabilidad de contacto de vecinos de la misma opinión y denotamos por $p_{d} \leq 1.0$ la probabilidad de contacto entre vecinos de opinión opuesta. Una vez que tienen un contacto físico, la infección se transmite con probabilidad $\beta$, lo que genera probabilidades de infección efectiva $\beta$ y $\beta p_{d} \leq \beta$ en cada caso.

En una situación donde hay un contacto físico pero no una conexión social entre dos vecinos (se ven pero no hablan de política), no se espera que las opiniones afecten (ni aumenten ni disminuyan) la probabilidad de contacto. Por lo tanto, esto puede considerarse como una situación intermedia respecto a los dos casos mencionados anteriormente, donde la probabilidad de contacto debería ser menor de 1.0 pero mayor que $p_{d}$, lo que daría lugar a una probabilidad de infección entre $\beta$ y $\beta p_{d}$. Sin embargo, para simplificar, suponemos que la probabilidad de contacto en ausencia de una relación social es la misma que en las relaciones homófilas (1.0) y, por lo tanto, la probabilidad de infección toma el valor $\beta$. Esta aproximación y la mencionada anteriormente para la probabilidad de interacción social tienen la ventaja de reducir el número de parámetros libres, lo que permite un análisis más profundo del modelo que ya exhibe un comportamiento muy rico como veremos. Ahora definimos la dinámica del modelo de acuerdo con las propiedades de interacción discutidas anteriormente. En un paso de tiempo $\Delta t=1 / N$ se intentan actualizar la opinión y la enfermedad en cada red, como se describe a continuación (Fig. 3.2).

Actualización de opinión [Fig. 3.2 (a)]: Un nodo $i$ con estado de opinión $\mathcal{O}_{i}$ y uno de sus vecinos $j$ con opinión $\mathcal{O}_{j}$ son elegidos al azar en la red social. Si $\mathcal{O}_{i}=\mathcal{O}_{j}$ no pasa nada. Si $\mathcal{O}_{i} \neq \mathcal{O}_{j}, i$ copia la opinión de $j\left(\mathcal{O}_{i} \rightarrow \mathcal{O}_{i}=\mathcal{O}_{j}\right)$ con probabilidad $p_{o}$ si hay un enlace de contacto entre $i$ y $j$, y al menos uno de los dos nodos está infectado $\left(\mathcal{D}_{i}=1\right.$ ó $\left.\mathcal{D}_{j}=1\right)$. De lo contrario, es decir, si no hay un enlace de contacto o $\mathcal{D}_{i}=\mathcal{D}_{j}=0$, entonces $i$ copia la opinión de $j$ con probabilidad 1.0.

Actualización de enfermedad [Fig. 3.2 (b)]: Un nodo $i$ con estado de enfermedad $\mathcal{D}_{i}$ se elige al azar en la red de contacto. Si $\mathcal{D}_{i}=0$ no pasa nada. Si $\mathcal{D}_{i}=1$, entonces $i$ se recupera con probabilidad $1-\beta$ o, con la probabilidad complementaria $\beta$ este nodo $(i)$ intentará infectar a un vecino elegido al azar $j$, siempre que este esté en el estado susceptible $\left(\mathcal{D}_{j}=0\right)$. La infección ocurre $\left(\mathcal{D}_{j}=0 \rightarrow \mathcal{D}_{j}=1\right)$ con probabilidad $p_{d}$ si hay un enlace social entre $i$ y $j$, y $\mathcal{O}_{i} \neq \mathcal{O}_{j}$. De lo contrario, es 

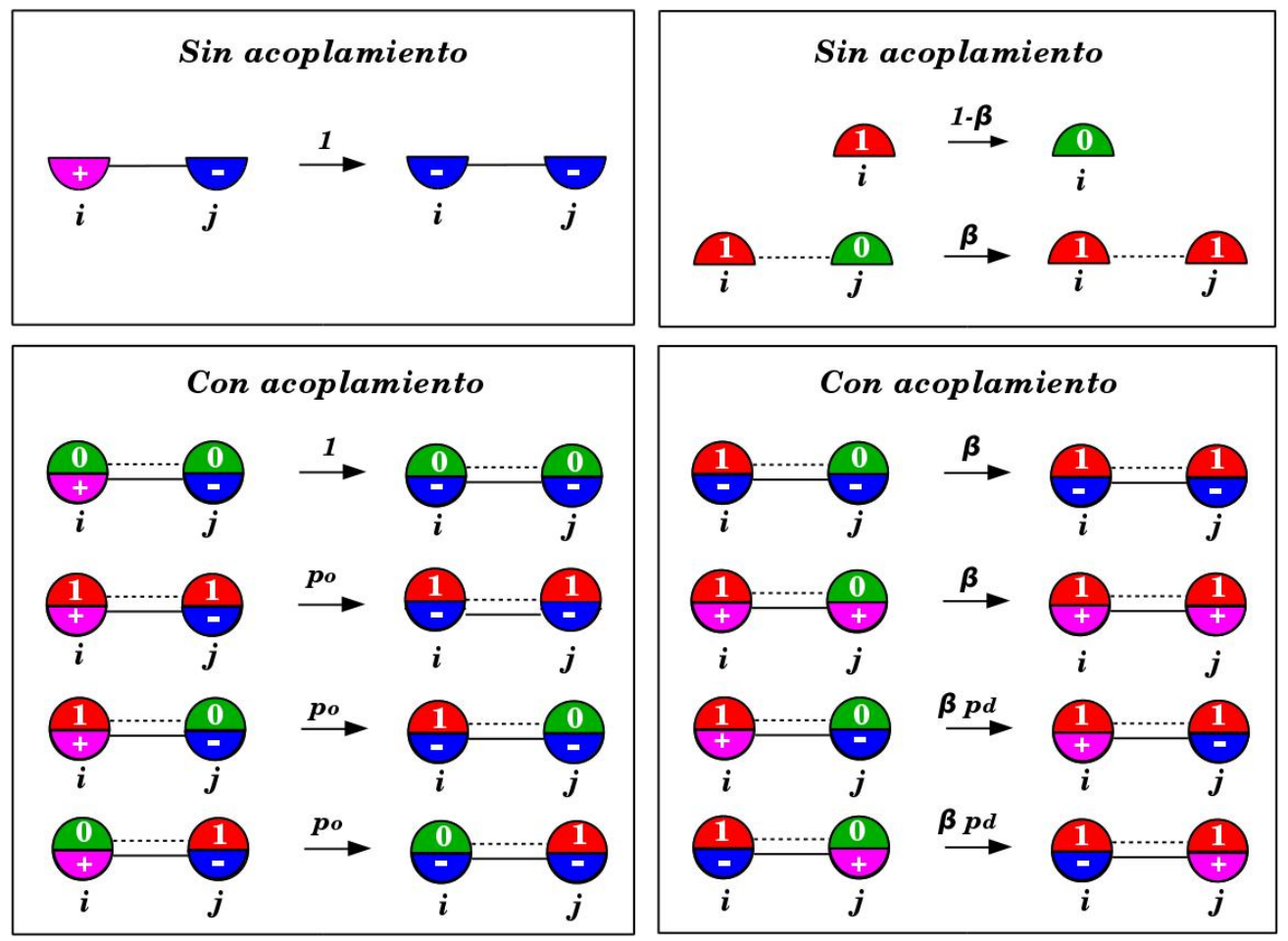

(a) Dinámica de Opinión

(b) Dinámica de Enfermedad

Figura 3.2: Actualización de las reglas del modelo en el sistema acoplado opiniónenfermedad. (a) Actualización de opinión: El nodo $i$ adopta la opinión de su vecino $j$ con probabilidad 1.0 cuando están conectados solo por un enlace social (línea continua). Cuando también están conectados por un enlace de contacto (línea punteada), la adopción ocurre con una probabilidad 1.0 si ambos nodos son susceptibles, y con probabilidad $p_{o} \leq 1.0$ si al menos un nodo está infectado. (b) Actualización de la enfermedad: Un nodo infectado $i$ se recupera con probabilidad $1-\beta$ o transmite la enfermedad a un vecino susceptible $j$ con probabilidad $\beta$ cuando ambos nodos solo están conectados por un enlace de contacto, o cuando están conectados por ambos tipos de enlaces y comparten la misma opinión. En caso de que tengan opiniones opuestas la transmisión ocurre con probabilidad $\beta p_{d} \leq \beta$.

decir, si no hay un enlace social ó $\mathcal{O}_{i}=\mathcal{O}_{j}$, el nodo $j$ se infectará con probabilidad 1.0 .

En otras palabras, los individuos en la capa social adoptan la opinión de sus vecinos con probabilidad 1.0 excepto cuando están conectados por un enlace de contacto y uno de ellos está infectado, donde en este caso la opinión es adoptada con una probabilidad reducida $p_{o} \leq 1$ (Fig. 3.2 (a)). La dinámica del CP ocurre en 
la capa de enfermedad con una probabilidad de infección $\beta$ entre dos vecinos, y se reduce a $\beta p_{d} \leq \beta$ solo en el caso de que estén conectados por un enlace social y compartan diferentes opiniones (Fig. $3.2(\mathrm{~b})$ ).

\subsection{Resultados Numéricos}

El CP y el VM son dos de los procesos dinámicos más estudiados 79. Una característica relevante del CP es la existencia de una transición de una fase sana a una fase endémica cuando la probabilidad de infección supera un valor umbral $\beta_{c}$ como se mencionó en la sección 1.4.1. La fase sana es estática, ya que todos los nodos son susceptibles y los eventos de infección no pueden ocurrir. La fase endémica es activa, donde cada nodo se somete a un ciclo infectado-susceptibleinfectado y la cantidad total de nodos infectados fluctúa alrededor de un valor estacionario. La transición sana-endémica es continua, y el valor crítico $\beta_{c}$ depende de las propiedades topológicas de la red [81]. Por su parte, el VM se ha utilizado ampliamente para explorar el consenso de opinión sobre diferentes topologías de red $6,92,96]$. Se encontró que las propiedades de difusión de las opiniones dependen de la heterogeneidad de la red. Esto se refleja en el tiempo medio de consenso, que es proporcional a la relación $\mu^{2} / \mu_{2}\left[6,96\right.$, donde $\mu$ y $\mu_{2}$ son el primer y segundo momento de la distribución de grado de la red.

El comportamiento descrito anteriormente es particular de cada modelo en redes aisladas. Para explorar cómo las propiedades de estos dos procesos se ven afectadas cuando se acoplan a través de una red múltiple, se ejecutaron simulaciones de Monte Carlo (MC) del modelo descrito en la sección 3.1, utilizando dos redes Erdös Rényi (ER) de grado medio $\langle k\rangle=\mu=10$ cada una. Inicialmente, cada nodo en el sistema está infectado con probabilidad 1/2, y adopta cualquiera de los estados de opinión + o - con la misma probabilidad 1/2. Es decir, el sistema comienza desde una condición inicial simétrica con aproximadamente $1 / 4$ de nodos en cada uno de los cuatro posibles estados de opinión-infección: $\left[\begin{array}{l}+ \\ 0\end{array}\right],\left[\begin{array}{l}+ \\ 1\end{array}\right],\left[\begin{array}{c}- \\ 0\end{array}\right]$ y $\left[\begin{array}{c}- \\ 1\end{array}\right]$.

En las siguientes dos subsecciones estudiamos por separado los efectos de una dinámica sobre la otra. 


\subsubsection{Efectos de la formación de opiniones sobre la preva- lencia de la enfermedad}

Comenzamos el análisis del modelo describiendo los resultados relacionados con los efectos de la formación de opiniones sobre las propiedades de la propagación de la enfermedad. En la Fig. 3.3 mostramos la fracción estacionaria de nodos infectados promediada sobre $10^{4}$ realizaciones independientes de la dinámica, $\left\langle\rho_{1}^{\text {stat }}\right\rangle$, como función de la probabilidad de infección $\beta$. Para este primer conjunto de simulaciones usamos $p_{o}=p_{d}=0$, que corresponde al escenario extremo donde las opiniones no pueden transmitirse a través de los vecinos de contacto (nodos conectados por un enlace de contacto) que están infectados, y las infecciones no están permitidas entre vecinos sociales (nodos conectados por un enlace social) con diferentes opiniones. Las diferentes curvas corresponden a diferentes valores del parámetro de acoplamiento $q$ y el tamaño de red $N$, como se indica en la leyenda. Observamos que, para $q=0.4$ (diamantes) y $q=0.7$ (triángulos), $\left\langle\rho_{1}^{\text {stat }}\right\rangle$ decrece suavemente con $\beta$ hasta un punto $\beta_{q}^{c}$ que depende de $q$, donde de repente decae a un valor cercano a cero. La disminución repentina en $\left\langle\rho_{1}^{\text {stat }}\right\rangle$ se vuelve más abrupta a medida que $N$ aumenta, lo que lleva a un cambio discontinuo de $\left\langle\rho_{1}^{\text {stat }}\right\rangle$ con $\beta_{q}^{c}$ en el límite termodinámico $(N \rightarrow \infty)$. Este comportamiento es una reminiscencia de una transición discontinua. También vemos que el salto en $\left\langle\rho_{1}^{\text {stat }}\right\rangle$ disminuye con $q$ y desaparece para el caso desacoplado $q=0$, donde la transición se vuelve continua, de acuerdo con el comportamiento conocido del CP en redes aisladas. El punto crítico $\beta_{0}^{c} \simeq 0.53$ para $q=0$ coincide muy bien con el encontrado en trabajos numéricos y analíticos anteriores 81 .

Estos resultados muestran que la dinámica de opiniones tiene un profundo efecto sobre las propiedades estadísticas de la propagación de la enfermedad, cambiando el tipo de transición de fase en el CP de una transición continua en ausencia de acoplamiento (cuando las dos dinámicas son independientes) a una transición discontinua cuando la dinámica está acoplada.

Con el fin de lograr una comprensión más profunda de la naturaleza de esta transición, estudiamos la evolución temporal de la fracción de nodos infectados $\rho_{1}(t)$ para el caso $q=0.4$, donde el punto de transición es $\beta_{0.4}^{c} \simeq 0.58$ (ver Fig. 3.3). Las líneas continuas en la Fig. 3.4 corresponden a los resultados para redes de tamaño $N=10^{4}$. Como podemos ver, para $\beta>\beta_{0.4}^{c} \simeq 0.58$ el valor promedio de $\rho_{1}(t)$ sobre $10^{4}$ realizaciones, $\left\langle\rho_{1}(t)\right\rangle$, varía no-monotónicamente con el tiempo y se 


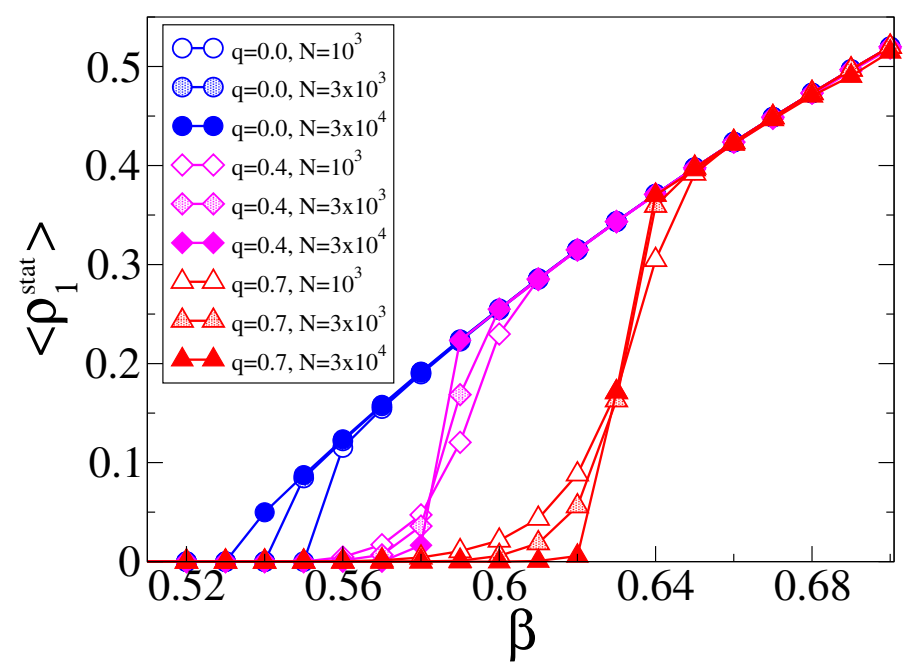

Figura 3.3: Fracción estacionaria promedio de los nodos infectados $\left\langle\rho_{1}^{\text {stat }}\right\rangle$ vs la probabilidad de infección $\beta$ en dos redes ER acopladas de grado medio $\mu=10$ y $N$ nodos cada una, para $p_{o}=p_{d}=0$ y parámetros de acoplamiento $q=0$ (círculos), $q=0.4$ (diamantes) y $q=0.7$ (triángulos). Los diferentes rellenos de los símbolos corresponden a tres tamaños diferentes del sistema $N=10^{3}, 3 \times 10^{3} \mathrm{y}$ $3 \times 10^{4}$, como se indica en la leyenda. El promedio fue de más de 5000 realizaciones independientes a partir de configuraciones que consisten en una fracción cercana al $50 \%$ de nodos infectados distribuidos uniformemente en la red de contacto y $50 \%$ de opiniones + uniformemente distribuidas a través de la red social.

acerca asintóticamente a un valor estacionario $\left\langle\rho_{1}^{\text {stat }}\right\rangle$ que depende de $\beta$, mientras que $\left\langle\rho_{1}(t)\right\rangle$ decae a cero para $\beta<\beta_{0.4}^{c}$. Es decir, esta no monotonía en $\left\langle\rho_{1}(t)\right\rangle$ hace a $\left\langle\rho_{1}^{\text {stat }}\right\rangle$ saltar desde un valor cercano a cero para $\beta<\beta_{0.4}^{c}\left(\left\langle\rho_{1}^{\text {stat }}\right\rangle \simeq 0.0014\right.$ para $\beta=0.57)$ a un valor mucho mayor $\operatorname{para} \beta>\beta_{0.4}^{c}\left(\left\langle\rho_{1}^{\text {stat }}\right\rangle \simeq 0.22\right.$ para $\beta=$ 0.59). Observamos que, como es conocido, este peculiar comportamiento temporal no monotónico induce transiciones discontinuas en modelos sociales con múltiples estados y restricciones, como el modelo de Axelrod ( 91,97$])$.

El origen del comportamiento no monótono de $\left\langle\rho_{1}^{\text {stat }}\right\rangle$ está en la naturaleza dinámica de la probabilidad de infección durante cada realización individual, que puede tomar dos valores posibles: el valor $\beta p_{d}=0$ a través de un enlace de contacto que se superpone con un enlace social +- , o el valor $\beta$ (las simulaciones corresponden a $\left.p_{d}=p_{o}=0\right)$. En otras palabras, la infectividad a través de un enlace determinado $i-j$ puede cambiar entre 0 y $\beta$ en el tiempo, dependiendo de los estados de opinión de los nodos $i$ y $j$. Esto proporciona una tasa de infección pro- 

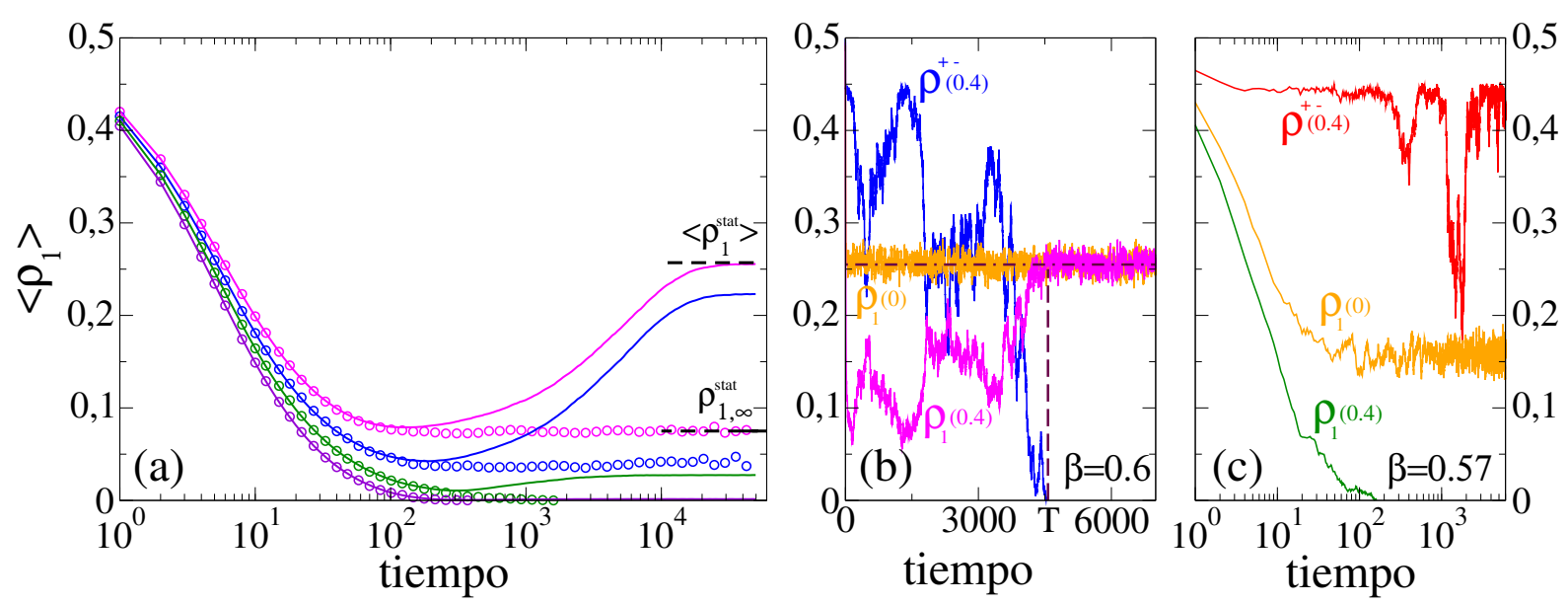

Figura 3.4: (a) Evolución temporal de la fracción promedio de nodos infectados $\left\langle\rho_{1}\right\rangle$ en un sistema bicapa con acoplamiento $q=0.4$. Las líneas sólidas corresponden a redes de tamaño $N=10^{4}$, mientras que los círculos abiertos son para redes con $N=10^{6}$ nodos. Las curvas corresponden a las probabilidades de infección $\beta=0.60$, $0.59,0.58$ y 0.57 (de arriba a abajo). Las líneas horizontales discontinuas indican los valores estacionarios de $\beta=0.60$ para los dos tamaños de red. (b) y (c) Evolución de la fracción de nodos infectados, $\rho_{1}$ y la fracción de enlaces sociales,$+- \rho^{+-}$, en dos realizaciones diferentes para $q=0.4$ y $\beta=0.6$ (b) y $\beta=0.57$ (c). La evolución de $\rho_{1}$ también se muestra para $q=0$ en ambos paneles.

medio sobre todo el sistema que fluctúa de acuerdo con la evolución de la fracción de enlaces,$+- \rho^{+-}(t)$, en una realización. Aprovecharemos esta observación en la sección 3.3 para desarrollar un enfoque de campo medio (MF) para la evolución del sistema.

En los paneles (b) y (c) de la Fig. 3.4 graficamos $\rho_{1}$ y $\rho^{+-}$en una sola realización de la dinámica, para $q=0.4$ y dos valores de $\beta$. Para $\beta=0.60>\beta_{0.4}^{c}$ [panel (b)] observamos que $\rho_{1}$ muestra grandes variaciones hasta un tiempo $T \simeq 4570$ (línea discontinua vertical) donde $\rho^{+-}$decae a 0 , después de lo cual $\rho_{1}$ fluctúa alrededor de un valor estacionario $\rho_{1}^{\text {stat }} \simeq 0.255$ (línea horizontal con el patrón $-\cdot-\cdot-$ ) ), mientras que para $\beta=0.57<\beta_{0.4}^{c}[$ panel $(\mathrm{c})] \rho_{1}$ decae rápidamente a cero, antes de que $\rho^{+-}$llegue a cero. Cuando $\rho^{+-}$se va a 0 [panel (b)] solo quedan enlaces ++ o - - y, por lo tanto, la dinámica de la enfermedad se comporta como la del CP estándar con probabilidad de infección $\beta=0.60$ en todos los enlaces, alcanzando el valor estacionario $\rho_{1}^{\text {stat }}(q=0, \beta=0.60) \simeq 0.255$. 


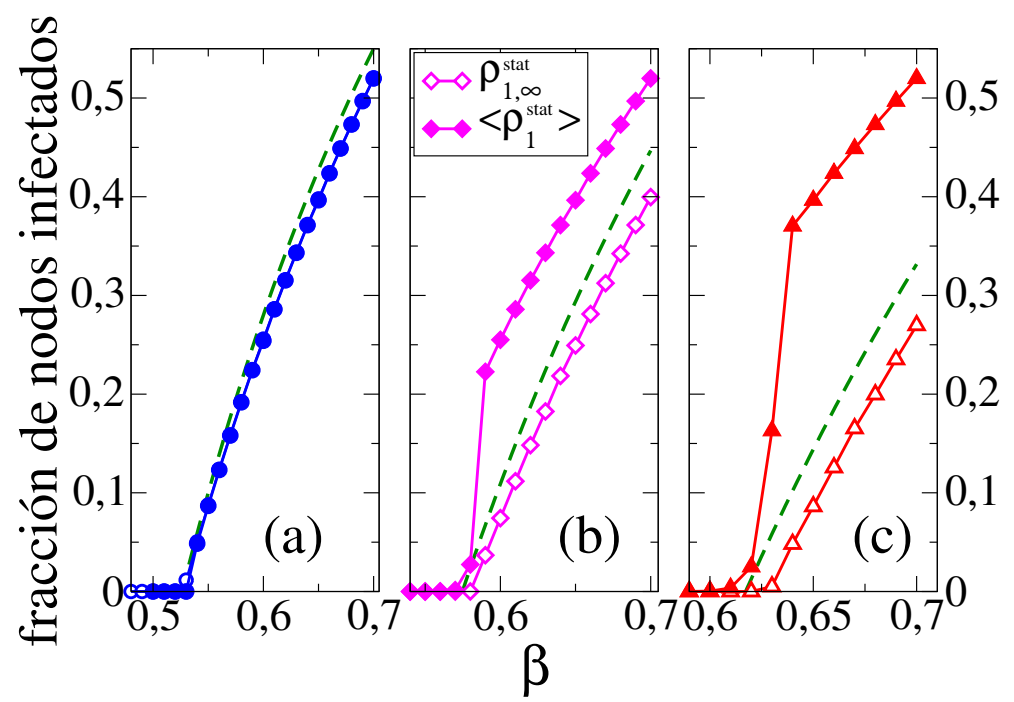

Figura 3.5: Fracción estacionaria de nodos infectados vs $\beta$, para los acoplamientos $q=0$ (a), $q=0.4$ (b) and $q=0.7$ (c). Los símbolos abiertos corresponden a realizaciones individuales en una red de tamaño $N=10^{6}$, mientras que los símbolos llenos corresponden a un promedio sobre 5000 realizaciones en redes de $N=10^{4}$ nodos. En el panel (a), los símbolos abiertos se superponen con los cerrados. La línea punteada representa la aproximación teórica de la Eq. (3.11).

Podemos decir que después de un tiempo $T$ la dinámica de la enfermedad se desacopla de la dinámica de opinión. De hecho, el panel (b) también muestra $\rho_{1}$ en una sola realización en una red aislada $(q=0)$ con $\beta=0.60$, donde observamos un decaimiento muy rápido a un valor estacionario que se superpone con el caso acoplado $q=0.4$. Por lo tanto, como podemos ver en la Fig. 3.3. el valor de $\rho_{1}^{\text {stat }}$ en la fase endémica del sistema acoplado $\left(\beta>\beta_{0.4}^{c}\right)$ es el mismo que en el caso desacoplado. Luego, en el punto de transición $\beta_{0.4}^{c} \simeq 0.58>\beta_{0}^{c} \simeq 0.53$, $\rho_{1}^{\text {stat }}$ salta desde el valor $\rho_{1}^{\text {stat }}\left(q=0, \beta_{0.4}^{c}\right) \simeq 0.22$ correspondiente al sistema desacoplado, a un pequeño valor $\rho_{1}^{\text {stat }} \simeq 0.027$, que muestra un cambio discontinuo. Este comportamiento particular de $\rho_{1}^{\text {stat }}$ es el origen de las transiciones discontinuas para $q>0$ que se muestran en la Fig. 3.3 .

En la sección 3.3 desarrollamos un enfoque de MF que permite estimar la fracción estacionaria de los nodos infectados $\rho_{1}$ (Ec. (3.11)). La aproximación teórica de la Ec. (3.11), que se muestra como curva discontinua en cada panel de la Fig. 3.5 , describe una transición continua con $\beta$, en contraste con la discontinuidad encontrada en simulaciones numéricas (símbolos llenos). Esto se debe a que el enfoque de MF supone un sistema infinitamente grande $(N=\infty)$ donde las fluctuaciones de 
tamaño finito no se toman en cuenta, mientras que las simulaciones corresponden al límite de sistemas muy grandes (pero aún finitos) $(N \gg 1)$. Las fluctuaciones en redes finitas conducen finalmente al sistema a un estado absorbente en el que todos los nodos son susceptibles $\left(\rho_{1}=0\right)$ y tienen una opinión $+\mathrm{o}-\left(\rho^{+-}=0\right)$, es decir, un consenso de opinión sobre una población completamente sana. Por lo tanto, las fluctuaciones juegan un papel fundamental en la naturaleza discontinua de la transición porque, como se discutió anteriormente, el valor estacionario de $\rho_{1}$ en una sola realización depende de si $\rho^{+-}$decae a cero antes de que $\rho_{1}$ lo haga.

Para obtener una mejor comprensión de los resultados obtenidos de la teoría MF, ejecutamos simulaciones en redes muy grandes. Los círculos abiertos en la Fig. 3.4 corresponden a realizaciones únicas en una red de $N=10^{6}$ nodos, para los mismos valores de $\beta$ utilizados para redes con $N=10^{4}$ nodos (líneas continuas). Observamos que las curvas de $N=10^{6}$ decaen monótonamente con el tiempo hasta un valor estacionario denotado por $\rho_{1, \infty}^{\text {stat }}$ (solo se muestra para $\beta=0,6$ ), que concuerda con el mínimo de las curvas no monótonas para $N=10^{4}$. Debemos tener en cuenta que estos estados no son realmente estacionarios, en el sentido de que $\rho_{1}$ exhibe un plateau muy largo (fuera de la escala mostrada) pero eventualmente aumenta y alcanza el mismo valor estacionario $\left\langle\rho_{1}{ }^{\text {stat }}\right\rangle$ de las curvas para $N=10^{4}$. Hemos comprobado que la longitud del plateau diverge con $N$ y, por lo tanto, es infinitamente grande cuando $N=\infty$. Por lo tanto, nosotros tomaremos $\rho_{1, \infty}^{\text {stat }}$ como el valor estacionario cuando $N=\infty$. En la Fig. 3.5 observamos que los valores numéricos $\rho_{1, \infty}^{\text {stat }}$ (símbolos abiertos) concuerdan bastante bien con la aproximación teórica de la ecuación (3.11) (curvas discontinuas) para los tres valores de $q$, aunque esta coincidencia empeora a medida que $q$ se hace más grande. También vemos que $\rho_{1, \infty}^{\text {stat }}$ decae continuamente a medida que $\beta$ disminuye y se va a cero en el mismo valor $\beta_{q}^{c}$ de la transición en el límite termodinámico correspondiente a $\rho_{1}^{\text {stat }}$ (símbolos llenos). Es decir, la transición sana-endémica es continua en un sistema infinito.

Hasta aquí estudiamos la respuesta del sistema cuando la probabilidad de infección varía para un acoplamiento fijo. Ahora exploramos los efectos de tener un acoplamiento variable en la prevalencia de la enfermedad. En la Fig. 3.6 (a) graficamos $\left\langle\rho_{1}^{\text {stat }}\right\rangle$ en dos redes acopladas de $N=10^{4}$ nodos (círculos) y $\rho_{1}^{\text {stat }}$ en una única realización en redes de tamaño $N=10^{6}$ (cuadrados), ambas en función del acoplamiento $q$, para $\beta=0.6$. La curva superior de $N=10^{4}$ muestra una transición abrupta de una fase endémica a una fase sana a medida que el acoplamiento supera un valor umbral $q_{0.6}^{c} \simeq 0.5$. 


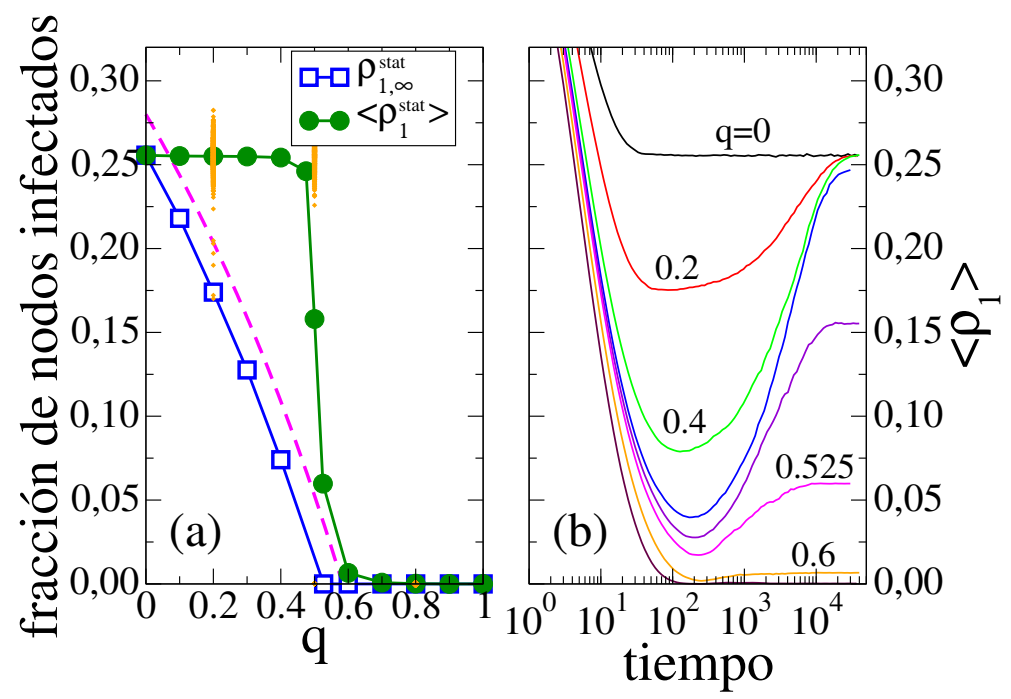

Figura 3.6: (a) Fracción estacionaria de nodos infectados $\rho_{1}^{\text {stat }}$ vs el acoplamiento $q$, para $\beta=0.6$. Los círculos llenos corresponden a un promedio de $\rho_{1}^{\text {stat }}$ sobre 5000 realizaciones en redes con $N=10^{4}$ nodos. Algunos valores de $\rho_{1}^{\text {stat }}$ en realizaciones independientes son mostrados por puntos para $q=0.2,0.5$ y 0.8 . Los cuadrados abiertos representan el resultado para $\rho_{1}^{\text {stat }}$ en una sola realización de una red de tamaño $N=10^{6}$. La línea punteada es la aproximación teórica de la Ec. (3.11). (b) Evolución temporal de $\left\langle\rho_{1}\right\rangle$ para valores de acoplamiento $q=0,0.2,0.4,0.475,0.5,0.525,0.6$ y 0.7 (de arriba hacia abajo) en redes de tamaño $N=10^{4}$.

Para explorar este comportamiento con más detalle, mostramos con puntos de color naranja el valor de $\rho_{1}^{\text {stat }}$ en cada realización individual para tres valores de $q$. Para $q=0.2$, todos los puntos caen alrededor de su valor medio $\left\langle\rho_{1}^{\text {stat }}\right\rangle \simeq 0.26$, mientras que para $q=0.8$ están en $\rho_{1}^{\text {stat }}=0$. En el punto de transición $q_{0.6}^{c}$ la distribución de puntos es bimodal, es decir, los puntos están alrededor de $\rho_{1}^{\text {stat }} \simeq 0.26$ y en $\rho_{1}^{\text {stat }}=0$, dando un valor promedio $\left\langle\rho_{1}^{\text {stat }}\right\rangle \simeq 0.165$. Esta es una evidencia de una transición discontinua. La razón de esta discontinuidad es la evolución temporal no monótona de $\left\langle\rho_{1}\right\rangle$ (Fig. 3.6 (b)), de forma similar a lo que ocurre cuando se varía $\beta$, como se mostró antes. La única diferencia entre ambos casos es que, como $\beta$ es fijo, el valor estacionario de $\rho_{1}$ en realizaciones individuales no cambia con $q$, pero es $\rho_{1}^{\text {stat }}=0$ ó $\rho_{1}^{\text {stat }} \simeq 0.26$, de acuerdo con la distribución binomial. La primera situación ocurre en realizaciones donde $\rho_{1}$ llega a cero antes que $\rho^{+-}$, mientras que la última situación corresponde a realizaciones donde $\rho^{+-}$decae rápidamente a cero y así las dos dinámicas se desacoplan, después de lo cual $\rho_{1}$ alcanza un valor 


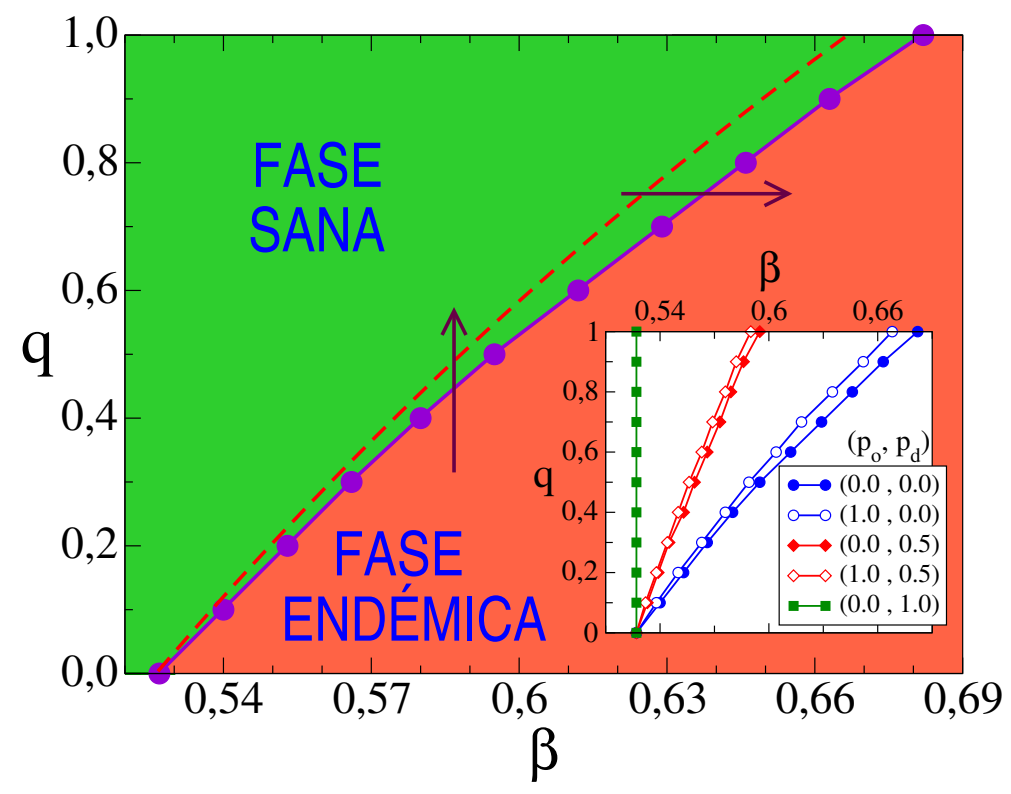

Figura 3.7: Diagrama de Fase del Proceso de Contacto (CP) acoplado con el Modelo del Votante (VM), mostrando las fases sana y endémica en el espacio $\beta-q$, con $p_{o}=p_{d}=0$. La línea punteada representa la aproximación analítica para la línea de transición dada por la Ec. (3.13). El gráfico interno muestra las líneas de transición para el conjunto de valores $\left(p_{o}, p_{d}\right)$ indicados en la leyenda. Para $p_{d}=1.0$ (cuadrados), la transición está dada por la línea vertical $\beta \simeq 0.53$ para todos los valores de $p_{o}$ (solo se muestra $p_{o}=0.0$ ).

estacionario similar a 0.26 correspondiente a $q=0$. La Fig. 3.6 (a) muestra que la transición con $q$ es continua en un sistema infinitamente grande (cuadrados). También se puede verificar que el valor estacionario $\rho_{1}^{\text {stat }}$ para un $q$ dado en un sistema infinito concuerda con el mínimo de la curva $\left\langle\rho_{1}\right\rangle$ vs tiempo de la Fig. 3.6 (b). Este comportamiento es similar al que se muestra en la Fig. 3.4 (a).

El diagrama de fase $\beta-q$ de la Fig. 3.7 resume los resultados obtenidos en esta sección, sobre cómo el acoplamiento entre las redes de contacto y social afecta la prevalencia de la enfermedad. Al aumentar el acoplamiento $q$, es posible llevar un sistema desacoplado inicialmente de la fase endémica a la sana (flecha vertical). Además, a medida que aumenta el acoplamiento, se necesita un mayor valor en la probabilidad de infección $\beta$ para pasar de la fase sana a la fase endémica (flecha horizontal).

Finalmente, se reprodujo el diagrama de fase para varios valores de la probabilidad $p_{d}$ de tener una infección a través de los enlaces +- , y la probabilidad $p_{o}$ 
de imitar la opinión entre vecinos infectados (Gráfico interno de la Fig. 3.7). Vemos que la orientación de la línea de transición que separa la fase sana de la endémica se vuelve más vertical a medida que aumenta $p_{d}$, ampliando la fase endémica, como es esperarse. Cuando $p_{d}=1.0$, la transición se vuelve independiente del acoplamiento $q$ y de $p_{o}$ (la curva es la misma para todos los valores de $p_{o}$ ). También observamos una ligera disminución de la fase sana cuando $p_{o}$ aumenta mientras se mantiene $\beta$ fijo. Como la fracción de enlaces +- disminuye más rápidamente cuando las opiniones se copian a mayor velocidad, se espera un aumento de la tasa de infección efectiva y, en consecuencia, una ampliación de la fase endémica.

\subsubsection{Efectos de la propagación enfermedades sobre el con- senso de opiniones}

En esta sección exploramos cómo la propagación de la enfermedad afecta la dinámica de las opiniones. Como la transmisión de opiniones entre nodos vecinos es más difícil cuando al menos uno de ellos está enfermo, estamos particularmente interesados en estudiar hasta qué punto la enfermedad ralentiza la difusión de opiniones sobre la red social, y cómo eso depende de $q, \beta, p_{o} \mathrm{y} p_{d}$. Una forma de cuantificar esto es mirando el momento en el que se alcanza el consenso de opinión. En la Fig. 3.8 mostramos cómo el tiempo medio de consenso $\tau$ varía con el acoplamiento $q$, para la probabilidad de infección $\beta=0.6$ y varios valores de $p_{o}$ y $p_{d}$. Para una mejor comparación con el Modelo del Votante en una red aislada, $\tau$ está normalizado por el tiempo medio de consenso $\tau_{0}$ cuando las redes están desacopladas $(q=0)$. Los símbolos corresponden a las simulaciones MC, mientras

que las líneas continuas son las aproximaciones analíticas de la Ec. (3.17) obtenidas en la sección 3.3 .

A continuación presentamos los resultados para $\beta$ sobre el punto crítico de una red aislada $\beta_{0}^{c} \simeq 0.53$ porque para $\beta<\beta_{0}^{c}$ los efectos de la enfermedad en tiempos de consenso son insignificantes. Esto ocurre porque para $\beta<\beta_{0}^{c}$ y cualquier valor de $q$ la enfermedad desaparece rápidamente en la red de contacto y, como todos los nodos son susceptibles, la dinámica de las opiniones se desacopla de la dinámica de la enfermedad, llegando a un consenso en un tiempo muy similar al del caso desacoplado $\left(\tau \simeq \tau_{0}\right)$.

En la Fig. 3.8 observamos que la $q$-dependencia de $\tau$ es bastante diversa, mostrando comportamientos monótonos y no monótonos. Esto es consecuencia de la 

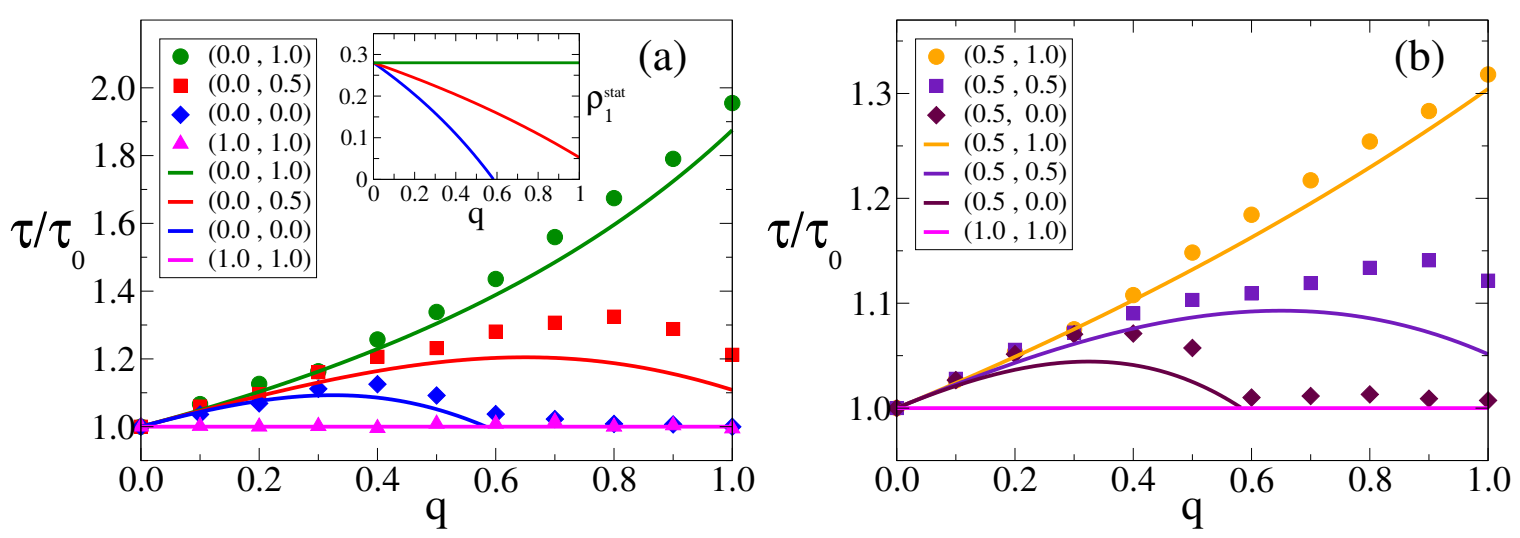

Figura 3.8: Tiempo medio $\tau$ para llegar al consenso de opinión sobre la red social como función del acoplamiento $q$ con la red de contacto, normalizado por el tiempo medio de consenso en ausencia del acoplamiento $\tau_{0}$. La probabilidad de infección en la red de contacto es $\beta=0.6$. Cada red tiene $N=10^{4}$ nodos y grado medio $\mu=10$. El promedio se hizo sobre 5000 realizaciones independientes. Los diferentes símbolos corresponden a resultados numéricos para el conjunto de valores $\left(p_{o}, p_{d}\right)$ indicados en las leyendas $\left((\mathrm{a})\right.$ para $p_{o}=0.0$ y (b) para $\left.p_{o}=0.5\right)$, mientras que las líneas continuas son las correspondientes aproximaciones analíticas de la Ec. (3.17). Para comparar, también mostramos en ambos paneles los datos numéricos y la curva analítica para el caso desacoplado $\left(p_{o}, p_{d}\right)=(1.0,1.0)$. Gráfico interno del panel (a): $\rho_{1}^{\text {stat }}$ vs $q$ de la Ec. (3.10) para $p_{d}=1.0,0.5$ y 0.0 (de arriba a abajo).

competencia entre dos mecanismos diferentes que afectan directamente la transmisión de la opinión. Una es la superposición de enlaces entre las dos redes que es proporcional a $q$, y la otra es la prevalencia de la enfermedad que disminuye con $q$, como explicaremos a continuación.

La transmisión de una opinión a través de un enlace social que se superpone con un enlace de contacto se ralentiza cuando al menos uno de los dos nodos está infectado y $p_{o}<1$. Por lo tanto, el retraso general en la transmisión de la opinión causada por la superposición total tiende a aumentar con $q$, y también lo hace $\tau$. Este efecto explica el aumento monotónico inicial de $\tau$ cuando $q$ aumenta desde 0 , en todas las curvas. Sin embargo, cuando $q$ se vuelve más grande, un segundo efecto se vuelve importante: la fracción de nodos infectados disminuye con $q$ (Gráfico interno de la Fig. 3.8 (a)), debido al acoplamiento con la dinámica de opinión que reduce la probabilidad de infección efectiva como se discutió en la sección 3.2.1. Luego, la 
disminución de la prevalencia de la enfermedad se traduce en menos enlaces sociales afectados por la enfermedad y, por lo tanto, en un menor atraso de opinión. Este efecto tiende a reducir $\tau$ con $q$.

Con estos dos mecanismos en juego, las formas de las curvas en la Fig. 3.8 para diferentes valores de $p_{o}$ y $p_{d}$ pueden explicarse cualitativamente en términos de los efectos combinados de la superposición (overlap) de enlaces y la prevalencia de la enfermedad. Por ejemplo, en la Fig. 3.8 (a) observamos que las tres curvas para $p_{o}=0.0$ tienen un comportamiento bastante diferente. Para $p_{d}=1.0$ el efecto de la prevalencia no varía con $q$, dado que $\rho_{1}^{\text {stat }}$ es independiente de $q$ (Gráfico interno de la Fig. $3.8(\mathrm{a}))$. Entonces, $\tau$ aumenta monótonamente con $q$ a medida que aumenta la superposición de enlaces. Para $p_{d}=0.5$ el efecto de la prevalencia aumenta con $q\left(\rho_{1}^{\text {stat }}\right.$ disminuye), llegando a dominar para valores de $q$ por encima de 0.8 cuando $\tau$ decae, y alcanzando un comportamiento no monótono de $\tau(q)$.

Finalmente, para $p_{0}=0.0$ observamos una no monotonicidad similar a la de la curva $p_{d}=0.5$, pero con la adición de que $\tau$ se vuelve muy similar a $\tau_{0}$ para todos los valores de $q>0.6$. Esto es porque $\rho_{1}^{\text {stat }}$ se va a cero por encima de $q \simeq 0.583$ y, por lo tanto, la enfermedad no tiene ningún efecto en las opiniones, lo que lleva a tiempos de consenso similares a los medidos en redes aisladas. Estos comportamientos para el caso $p_{o}=0.0$ también se observan para otros valores de $p_{o}$, como se muestra en la Fig. 3.8 (b) $\left(p_{o}=0.5\right)$. Vemos que la forma de las curvas para $p_{d}=0.0,0.5$ y 1.0 son análogas a las de Fig. 3.8 (a) para los valores correspondientes de $p_{d}$. Sin embargo, los tiempos de consenso son menores para el caso $p_{o}=0.5$ porque el atraso en la transmisión de la opinión se reduce a medida que aumenta $p_{o}$.

En la Fig. 3.9 graficamos el tiempo medio de consenso normalizado $\tau / \tau_{0}$ como función de la probabilidad de infección $\beta$ obtenida de la Ec. (3.17) . Los paneles (a) y (b) corresponden a acoplamientos $q=0.5$ y $q=1.0$, respectivamente. Para analizar estos gráficos recordamos que, como se explicó anteriormente, los tiempos de consenso aumentan con el nivel de prevalencia de la enfermedad en la red de contacto, dado que una mayor prevalencia de la enfermedad se traduce en un mayor retraso en la propagación de la opinión y en el consenso posterior. Una primera observación simple es que $\tau$ aumenta con $\beta$ y también con $p_{d}$, como se espera del hecho que un valor mayor de $\beta$ y $p_{d}$ implica una mayor prevalencia de la enfermedad. Una segunda observación es que $\tau$ disminuye con la probabilidad de transmisión de opinión $p_{o}$, como se explicó antes cuando comparamos $\tau$ en la Fig. 3.8 (a) con la 

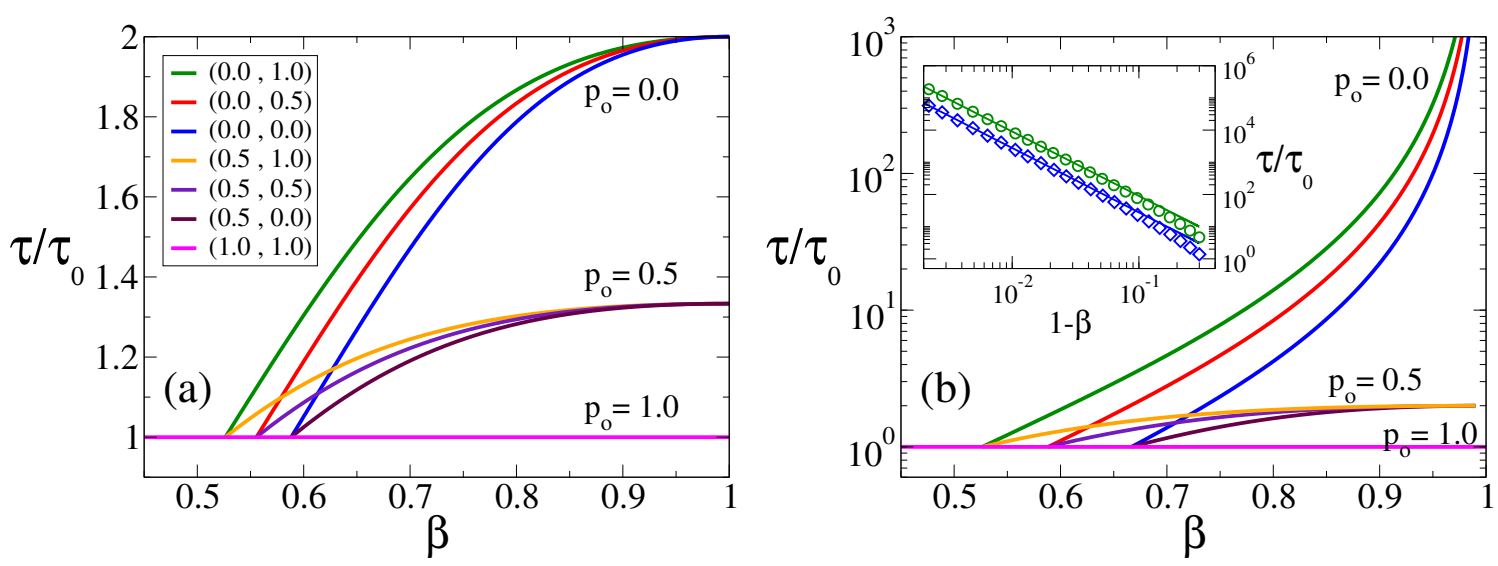

Figura 3.9: Tiempo medio de consenso $\tau / \tau_{0}$ en la red social vs. la probabilidad de infección $\beta$ en la red de contactos para los mismos parámetros de red que en la Fig. 3.8, el acoplamiento $q=0.5$ (a) y $q=1.0$ (b). Las curvas corresponden a la Ec. (3.17) para diferentes valores del conjunto $\left(p_{o}, p_{d}\right)$ con el mismo código de color que en la Fig. 3.8, y se indican en la leyenda del panel (a). Las curvas para los conjuntos $(1.0,0.0)$ y $(1.0,0.5)$ se superponen con la curva de $(1.0,1.0)$ que se muestra como la línea horizontal $\tau / \tau_{0}=1.0$. El gráfico interno del panel (b) muestra la divergencia de $\tau$ con $\beta$ aproximándose a 1.0 , cuando $q=1.0, p_{o}=0.0 \mathrm{y}$ $p_{d}=1.0$ (círculos) y $p_{d}=0.0$ (diamantes). Las líneas continuas son aproximaciones de la Ec. 3.17.

Fig. 3.8 (b). Una tercera observación es que $\tau$ se aproxima a un valor independiente de $p_{d}$ cuando $\beta$ se aproxima a 1.0. Esto es porque para $\beta=1.0$ (la probabilidad de recuperación es igual a cero) y para un valor fijo de $q$ y $p_{o}$ todos los nodos están infectados en el estado estacionario, independientemente del valor de $p_{d} \mathrm{y}$, por lo tanto, los tiempos de consenso son iguales para todos los valores de $p_{d}$. Como vemos en la Fig. 3.9 (b), el caso $q=1.0$ y $p_{o}=0.0$ es especial porque $\tau$ diverge cuando $\beta$ se acerca a 1.0. Esto sucede porque en esta situación, la transmisión de opiniones solo es posible entre nodos conectados que son susceptibles, y desaparecen en el límite de $\beta \rightarrow 1.0$, lo que lleva a tiempos de consenso divergentes. Se puede obtener una estimación aproximada de cómo $\tau$ escalea con $\beta$ suponiendo que $\tau$ es proporcional a la escala de tiempo asociada a la transmisión de opinión a través de dos nodos vecinos en la red social, $i$ y $j$, con opiniones $+\mathrm{y}-$, respectivamente. Como $q=1$, $i$ y $j$ también son vecinos en la red de contacto. A partir de una situación en la que $i$ y $j$ están infectados por un alto valor de $\beta$, la transmisión de opinión ocurre 
después de que ambos nodos se recuperan. Por lo tanto, $\tau$ se determina cuando el enlace de $1-1$ se convierte en un enlace de $0-0$, que escalea como $(1-\beta)^{-2}$. En la próxima sección 3.3 derivamos una expresión más precisa para $\tau$ que muestra esta divergencia cuadrática en el límite de $\beta \rightarrow 1$, que se muestra en el gráfico interno de la Fig. 3.9 (b) con líneas continuas.

\subsection{Aproximación analítica}

Para obtener una idea del comportamiento del sistema de dos capas descrito en la sección 3.2, desarrollamos un enfoque de campo medio (MF) que permite estudiar la evolución temporal del sistema en términos de las densidades globales de nodos y enlaces en diferentes estados. Denotamos como $\rho^{+}$y $\rho^{-}$a las fracciones de nodos con opiniones $+\mathrm{y}-$ en la red social, respectivamente, y como $\rho_{1}$ y $\rho_{0}$ a las fracciones de nodos infectados y susceptibles en la red de contacto, respectivamente. Las fracciones de enlaces sociales entre nodos con opiniones $+\mathrm{y}-$ se denota con $\rho^{+-}$, mientras que $\rho_{10}$ representa la fracción de enlaces de contacto entre nodos infectados y susceptibles. Se usa una notación análoga para los enlaces sociales ++ y - - y para los enlaces de contacto 1-1 y $0-0$. Las fracciones de los nodos $\rho^{+}$y $\rho_{1}$ se normalizan con respecto al número de nodos totales $N$ en cada red, mientras que las fracciones de los enlaces $\rho^{+-}$y $\rho_{10}$ se normalizan por el número total de enlaces $\mu N / 2$ en cada red, con grado medio $\mu=\langle k\rangle$. Dado que la cantidad de nodos y enlaces se conserva en cada capa, las siguientes relaciones de conservación se mantienen en cualquier momento para la red social:

$$
\begin{aligned}
1 & =\rho^{+}+\rho^{-}, \\
1 & =\rho^{++}+\rho^{--}+\rho^{+-}, \\
\rho^{+} & =\rho^{++}+\frac{1}{2} \rho^{+-}, \\
\rho^{-} & =\rho^{--}+\frac{1}{2} \rho^{+-},
\end{aligned}
$$

y de forma análoga para la red de contacto:

$$
\begin{aligned}
1 & =\rho_{1}+\rho_{0}, \\
1 & =\rho_{11}+\rho_{00}+\rho_{10}, \\
\rho_{1} & =\rho_{11}+\frac{1}{2} \rho_{10}, \\
\rho_{0} & =\rho_{00}+\frac{1}{2} \rho_{10} .
\end{aligned}
$$


En los apéndices C, D, E y E desarrollamos un enfoque de campo medio que permite obtener el siguiente sistema de ecuaciones diferenciales acopladas para $\rho^{+}$, $\rho^{+-}, \rho_{1}$ y $\rho_{10}$, respectivamente:

$$
\begin{aligned}
\frac{d \rho^{+}}{d t} & =0 \\
\frac{d \rho^{+-}}{d t} & =\frac{2 \omega \rho^{+-}}{\mu}\left[(\mu-1)\left(1-\frac{\rho^{+-}}{2 \rho^{+}\left(1-\rho^{+}\right)}\right)-1\right],
\end{aligned}
$$

con

$$
\omega \equiv 1-q\left(1-p_{o}\right)\left(\rho_{1}+\frac{\rho_{10}}{2}\right)
$$

y

$$
\begin{aligned}
\frac{d \rho_{1}}{d t} & =\frac{\gamma \beta \rho_{10}}{2}-(1-\beta) \rho_{1} \\
\frac{d \rho_{10}}{d t} & =\frac{\gamma \beta \rho_{10}}{\mu}\left[(\mu-1)\left(1-\frac{\rho_{10}}{1-\rho_{1}}\right)-1\right]+2(1-\beta)\left(\rho_{1}-\rho_{10}\right),
\end{aligned}
$$

con

$$
\gamma \equiv 1-q\left(1-p_{d}\right) \rho^{+-}
$$

Estas ecuaciones representan una descripción matemática aproximada de la evolución temporal del modelo en redes infinitamente grandes, donde no se toman en cuenta las fluctuaciones por tamaño finito. Observamos que las Ecs. (3.3) y las Ecs. (3.5) se acoplan a través de los prefactores $\omega$ y $\gamma$, que dependen del acoplamiento $q$ y describen la dinámica de la opinión y de la enfermedad, respectivamente.

El/la lector/a interesado/a puede encontrar en los apéndices los detalles de la derivación de estas ecuaciones. En aras de la simplicidad, supusimos en la derivación que todos los nodos tienen el mismo número de vecinos $k=\mu$ elegidos al azar, lo que equivale a suponer que las redes son grafos aleatorios de grado regular. Sin embargo, esperamos que esta aproximación funcione bien en redes con distribuciones de grado homogéneas como las redes ER que utilizamos en las simulaciones MC. También implementamos una aproximación de pares homogénea [6] que tiene en cuenta las correlaciones entre el estado de nodos vecinos dentro de la misma capa (aproximación de pares intracapas), pero descuida las correlaciones entre estados de opinión y de enfermedad de ambas capas (aproximación de pares intercapas). Es decir, consideramos que el estado de opinión de cada nodo no está correlacionado con su propio estado de enfermedad y con los estados de enfermedad de sus vecinos 
y a la inversa, que su estado de enfermedad no está correlacionado con su propio estado de opinión y el de sus vecinos.

Es instructivo analizar la estructura de las Ecs. (3.3) y (3.5). Las Ecs. 3.3 describen la evolución de las opiniones en la capa social. De la Ec. (3.3a) vemos que la fracción de nodos + se conserva en el tiempo: $\rho^{+}(t)=\rho^{+}(t=0)$ para $t \geq 0$. Este comportamiento es una reminiscencia del VM en topologías aisladas, donde las densidades de opinión se conservan en cada paso de tiempo. Parece que la dinámica de la enfermedad no puede romper la simetría intrínseca de los estados de opinión inducidos por la dinámica del votante. La Ec. (3.3b) para la evolución de $\rho^{+-}$tiene un prefactor extra $\omega$ comparado con la ecuación correspondiente para el VM en redes aisladas [6], que muestra que la enfermedad afecta la dinámica de las opiniones a través de su nivel de prevalencia, expresada por $\rho_{1}$ y $\rho_{10}$ (Ec. (3.4)). Como se discute en el apéndice C, $\omega$ puede interpretarse como la "probabilidad efectiva" de que un nodo $i$ adopte la opinión de un vecino social $j$ elegido con opinión opuesta, que depende del estado de enfermedad de $i$ y $j$. Dentro del enfoque de MF, podemos suponer que la probabilidad de que $i$ copie a $j$ dependa del estado de enfermedad de un "par promedio" de vecinos de contacto, y que esta probabilidad sea la misma para todos los vecinos sociales. En estos términos, $\omega$ se convierte en la probabilidad media de copiado en toda la red social. De hecho, podemos verificar que el valor promedio de $\omega$ sobre las tres configuraciones posibles de conexión y estado de enfermedad de un par de contactos es

probabilidad de copiar $= \begin{cases}1 & \text { con prob. } 1-q(\text { sin link de contacto }), \\ 1 & \text { con prob. } q \rho_{00}(00 \text { link de contacto }), \\ p_{o} & \text { con prob. } q\left(1-\rho_{00}\right)(10,01 \text { ó } 11 \text { link de contacto }),\end{cases}$ esto da como resultado $\omega=1-q+q\left[\rho_{00}+\left(1-\rho_{00}\right) p_{o}\right]$, que se reduce a la Ec. (3.4) usando la relación $1-\rho_{00}=\rho_{1}+\rho_{10} / 2$ que sigue de las Ecs. (3.2b) y $3.2 \mathrm{c}$. Como vemos, el efecto general de la enfermedad en la dinámica de opinión a nivel de MF es reducir en un factor $\omega$ la velocidad a la que las opiniones cambian en cada nodo. Este efecto ralentiza la propagación de opiniones a través de la red social, pero no parece alterar las propiedades de la dinámica del votante.

Las Ecs. 3.5 describen la evolución de la enfermedad en la capa de contacto. Estas ecuaciones tienen la misma forma que las ecuaciones correspondientes para el CP en una red aislada dentro de la aproximación de pares homogénea [81], pero con una probabilidad de infección dada por $\gamma \beta \leq \beta$. En analogía al caso de $\omega$ 
descrito anteriormente, $\gamma \beta$ puede interpretarse como la "probabilidad efectiva" de que un dado nodo infectado $i$ transmita la enfermedad a un vecino susceptible $j$ en la capa de contacto, que depende de las opiniones de $i$ y $j$. De hecho, la expresión $\gamma \beta=\left[1-q\left(1-p_{d}\right) \rho^{+-}\right] \beta$ de la Ec. (3.6) es la probabilidad promedio de infección en la red de contacto, calculado sobre las tres configuraciones posibles de conexión y estado de opinión de un par social:

$$
\text { probabilidad de infección }= \begin{cases}\beta & \text { con prob. } 1-q(\text { sin link social }), \\ \beta & \text { con prob. } q\left(1-\rho^{+-}\right) \\ & (\text {cualquier link social }++ \text { o }--) \\ \beta p_{d} & \text { con prob. } q \rho^{+-}(+- \text {link social }) .\end{cases}
$$

Por lo tanto, nuestro enfoque de MF supone que esta "probabilidad efectiva de infección" de $i$ a $j$ depende de los estados de opinión de un "par promedio" de vecinos en la red social, y eso es lo mismo para todos los vecinos de la red de contacto. Podemos decir que, a nivel de MF, la dinámica de la enfermedad sigue al CP estándar en una única red aislada con probabilidad de infección homogénea $\gamma \beta$ y probabilidad de recuperación $1-\beta$ en cada nodo. Por lo tanto, la dinámica de opiniones tiene un efecto sobre la dinámica de la enfermedad equivalente a la de un campo externo homogéneo que actúa sobre cada nodo de la red de contacto, reduciendo la probabilidad de infección entre vecinos por un factor $\gamma$, manteniendo la misma probabilidad de recuperación.

En las dos subsecciones siguientes derivamos las expresiones analíticas para la prevalencia de la enfermedad $\rho_{1}^{\text {stat }}$ y el tiempo medio de consenso $\tau$, del sistema de Ecs. $3.3-3.6$.

\subsubsection{Prevalencia de la enfermedad}

Para estudiar cómo la dinámica de opinión afecta la prevalencia de la enfermedad, derivaremos la expresión para la fracción de nodos infectados en el estado estacionario $\rho_{1}^{\text {stat }}$ de las Ecs. 3.3$)$ y (3.5). Comenzaremos igualando las cuatro derivadas a cero, y luego, sustituiremos $\rho_{10}$ por $2(1-\beta) \rho_{1} / \gamma \beta$ de la Ec. (3.5a) en la Ec. 3.5b y resolveremos para $\rho_{1}$. Después de hacer algo de álgebra, obtenemos dos soluciones, pero solo una es estable dependiendo de los valores de los parámetros. 
La solución no trivial

$$
\rho_{1}^{\text {stat }}=\frac{[(\mu-1) \gamma+\mu] \beta-\mu}{[(\mu-1) \gamma+1] \beta-1}
$$

corresponde a la fase endémica, donde una fracción de nodos está infectada, y solo es estable cuando el numerador $\lambda \equiv[(\mu-1) \gamma+\mu] \beta-\mu$ es mayor que cero. Para $\lambda<0$ la solución estable es $\rho_{1}^{\text {stat }}=0$, correspondiente a la fase sana donde todos los nodos son susceptibles, mientras que $\lambda=0$ indica el punto de transición entre la fase endémica y la saludable. La expresión para $\rho_{1}^{\text {stat }}$ de la Ec. (3.7) aún no está cerrada porque depende de $\rho^{+-}$, a través del prefactor $\gamma$. De la Ec. 3.3b vemos que la fracción de enlaces sociales +- alcanza un valor estacionario dado por la expresión

$$
\rho_{\text {stat }}^{+-}=\frac{2(\mu-2)}{(\mu-1)} \rho^{+}(0)\left[1-\rho^{+}(0)\right],
$$

donde usamos $\rho^{+}=\rho^{+}(0)$ dado que $\rho^{+}$permanece constante en el tiempo, como se mencionó anteriormente. Notamos que $\omega$ no afecta el valor estacionario de $\rho^{+-}$, que sigue siendo el mismo que en el VM original [6]. Para una condición inicial simétrica en la red social $\left(\rho^{+}(0)=1 / 2\right)$, como la utilizada en las simulaciones, tenemos $\rho_{\text {stat }}^{+-}=(\mu-2) /[2(\mu-1)]$. Sustituyendo esta última expresión para $\rho_{\text {stat }}^{+-}$en la Ec. (3.6) obtenemos la siguiente expresión para $\gamma$ :

$$
\gamma=1-\frac{q\left(1-p_{d}\right)(\mu-2)}{2(\mu-1)} .
$$

Finalmente, sustituyendo la Ec. (3.9) en Ec. (3.7) llegamos a la siguiente expresión aproximada para la fracción estacionaria de los nodos infectados en la fase endémica:

$$
\rho_{1}^{\mathrm{stat}}=\frac{\left[2(2 \mu-1)-q\left(1-p_{d}\right)(\mu-2)\right] \beta-2 \mu}{\left[2 \mu-q\left(1-p_{d}\right)(\mu-2)\right] \beta-2} .
$$

Para una red de grado medio $\mu=10$ y $p_{d}=0$, la Ec. (3.10) se reduce a la simple expresión

$$
\rho_{1}^{\text {stat }}=\frac{(19-4 q) \beta-10}{(10-4 q) \beta-1}
$$

que es graficada en las Figs. 3.5 y 3.6 (líneas punteadas). Como la teoría de MF está pensada para trabajar en sistemas infinitamente grandes, también graficamos para comparar los resultados numéricos obtenidos de simulaciones para redes muy grandes (símbolos abiertos). Observamos que, en todos los casos, el valor teórico estimado de la fracción de nodos infectados de la ecuación (3.11) es mayor que el de las simulaciones. Esto se debe al hecho de que el enfoque de MF no tiene en cuenta las correlaciones entre los estados de opinión y los estados de enfermedad. 
Un evento de infección de $0 \rightarrow 1$ entre dos vecinos conectados por un enlace social y de contacto solo es posible cuando los estados de los nodos son $\left[\begin{array}{c}+ \\ 1\end{array}\right]$ y $\left[\begin{array}{c}+ \\ 0\end{array}\right]$ (un par $\left[\begin{array}{l}++ \\ 10\end{array}\right]$ ) ó $\left[\begin{array}{c}- \\ 1\end{array}\right]$ y $\left[\begin{array}{c}- \\ 0\end{array}\right]$ (un par $\left[\begin{array}{l}-- \\ 10\end{array}\right]$ ), porque $p_{d}=0$ en las Figs. 3.5 y 3.6 Entonces, se espera que los enlaces sociales ++ se correlacionen negativamente con enlaces 10 y se correlacionen positivamente con enlaces 11 y 00, dado que los vecinos de la misma opinión tienden a infectarse unos a otros y, por lo tanto, en un momento dado, es más probable que ambos estén infectados o que ambos estén susceptibles.

Sin embargo, esta aproximación teórica asume que los enlaces sociales ++ no están correlacionados con enlaces de contacto 10 (ver apéndice C) y, por lo tanto, la probabilidad estimada de encontrar un par $\left[\begin{array}{l}++ \\ 1\end{array}\right]$ es más grande que el obtenido cuando se consideran las correlaciones negativas. La misma conclusión también se cumple para los pares $\left[\begin{array}{c}-- \\ 1\end{array}\right]$. Esto lleva a una sobreestimación teórica de la cantidad de pares $\left[\begin{array}{l}++ \\ 1\end{array}\right]$ y $\left[\begin{array}{l}-- \\ 1\end{array}\right]$ y, en consecuencia, a una mayor tasa de infecciones que aumenta la prevalencia de la enfermedad con respecto a los resultados numéricos, como vemos en las Figs. 3.5 y 3.6 .

La Fig. 3.5 muestra que la forma de la Ec. 3.11 para $\rho_{1}^{\text {stat }}$ decrece continuamente y desaparece a medida que $\beta$ disminuye más allá de un valor umbral, como ocurre en el CP estándar. Esto muestra que la transición al estado sano es continua dentro del enfoque de MF, que supone que el sistema es infinitamente grande. En la Fig. 3.6 vemos que $\rho_{1}^{\text {stat }}$ disminuye con $q$, reduciendo la prevalencia e induciendo una transición a la fase sana. Es decir, la Ec. 3.10 predice una transición continua endémica-sana ya que $\beta$ y $q$ varían, esto ocurre en el punto donde $\rho_{1}^{\text {stat }}$ desaparece, lo que lleva a la relación

$$
\left[2(2 \mu-1)-q_{c}\left(1-p_{d}\right)(\mu-2)\right] \beta_{c}-2 \mu=0 .
$$

La línea de transición

$$
q_{c}=\frac{19 \beta_{c}-10}{4\left(1-p_{d}\right) \beta_{c}}
$$

obtenida de la Ec. 3.12 para $\mu=10$ se grafica en la Fig. 3.7 para $p_{d}=0$ (curva punteada). Podemos ver que la coincidencia con las simulaciones es buena para valores pequeños del acoplamiento $q$, pero surgen discrepancias a medida que aumenta $q$, donde la predicción teórica de la Ec. (3.13) sobreestima los valores numéricos. Otra observación simple que se desprende de la Ec. (3.13) es que para $\beta>10 /\left[19-4\left(1-p_{d}\right)\right]$ obtenemos el valor no físico $q_{c}>1$. Esto significa que, en el 
modelo de red, es posible inducir una transición aumentando el acoplamiento solo cuando $\beta$ es menor que un valor dado, como lo vemos en la Fig. 3.7 para $\beta<0.68$.

Como observación final hacemos hincapié en que las transiciones dentro de este enfoque de MF son continuas, de acuerdo con las simulaciones en redes muy grandes. Esto es así ya que las Ecs. (3.3) y (3.5) corresponden a un sistema infinito en el que no se toman en cuenta las fluctuaciones por tamaño finito.

\subsubsection{Tiempos de consenso de opiniones}

En esta sección estudiamos los efectos cuantitativos de la enfermedad en el tiempo para llegar al consenso de opinión. Para eso, proponemos una estimación analítica del tiempo medio de consenso $\tau$ en función de los parámetros del modelo.

Como se menciona en la sección 3.3 , en sistemas infinitamente grandes, $\rho^{+}$ permanece constante a lo largo del tiempo [Ec. (3.3a)]. Sin embargo, en sistemas finitos $\rho^{+}$fluctúa hasta que alcanza cualquier valor $\rho^{+}=1($ consenso +$)$ ó $\rho^{+}=0$ (consenso -), con ambas configuraciones caracterizadas por la ausencia de enlaces sociales $+-\left(\rho^{+-}=0\right)$. Una evolución típica de $\rho^{+-}$hacia el estado absorbente se puede ver en la Fig. 3.4 (b) para $q=0.4$ en redes con $N=10^{4}$ nodos. Es decir, finalmente se logra el consenso en los sistemas finitos debido a la naturaleza estocástica de la dinámica de opinión, que lleva a la red social a un estado donde todos los nodos comparten la misma opinión.

En una única opinión, la actualización de $\rho^{+}$puede aumentar o disminuir en $1 / N$ con la misma probabilidad $\omega \rho^{+-} / 2$, calculada como la probabilidad $\rho^{+-} / 2$ en que un nodo y un vecino de opinión opuesta se seleccionan al azar, multiplicado por la probabilidad $\omega$ de adopción de opinión. Por lo tanto, la dinámica estocástica del VM se puede estudiar asignando $\rho^{+}$a la posición de un caminante aleatorio simétrico unidimensional en el intervalo $[0,1]$, con una probabilidad de salto proporcional a $\omega \rho_{\text {stat }}^{+-} / 2$ y una longitud de paso de $1 / N$. A partir de una configuración simétrica con $N / 2$ nodos con opinión $+\left(\rho^{+}(0)=1 / 2\right)$, el caminante alcanza cualquiera de los puntos absorbentes $\rho^{+}=1$ ó $\rho^{+}=0$ en un número promedio de pasos que se escalea como $N^{2}$. Luego, dado que el caminate realiza un solo paso en un número promedio de intentos que escalea como $1 / \omega \rho_{\text {stat }}^{+-}$, y que el tiempo aumenta en $1 / N$ 
en cada intento, encontramos que el tiempo medio de consenso escalea como

$$
\tau \sim \frac{N}{\omega \rho_{\text {stat }}^{+-}} .
$$

Como vemos en la Ec. (3.8), $\rho_{\text {stat }}^{+-}$es independiente de la prevalencia de la enfermedad $\rho_{1} \mathrm{y}$, por lo tanto, la prevalencia afecta a $\tau$ solo a través de la probabilidad de copiar efectiva $\omega$, que establece la escala de tiempo asociada a las actualizaciones de opinión. De la Ec. (3.4) vemos que $\omega$ es igual a 1.0 cuando las redes están desacopladas $(q=0)$ o cuando $p_{o}=1.0$, y así la dinámica de las opiniones es exactamente igual que la del VM original. Sin embargo, $\omega$ es menor que 1.0 en presencia de acoplamiento $(q>0)$ y $p_{o}<1.0$, por lo tanto la evolución de la dinámica es "ralentizada" - en promedio- por un factor $1 / \omega>1.0$, dado que las opiniones se copian a una tasa que es $\omega$ veces menor que en el caso desacoplado. Como consecuencia, $\tau$ aumenta por un factor de $1 / \omega$ con respecto al tiempo medio de consenso en el caso desacoplado $\tau_{0}=\tau(q=0) \sim N / \rho_{\text {stat }}^{+-}$, esto es

$$
\frac{\tau}{\tau_{0}} \simeq \frac{1}{\omega}
$$

Para obtener una expresión completa para el radio $\tau / \tau_{0}$ como función de los parámetros del modelo, expresamos $\omega$ en términos de $\rho_{1}^{\text {stat }}$, sustituyendo en la Ec. (3.4) el valor estacionario de $\rho_{10}$ que sigue la Ec. (3.5a), $\rho_{10}^{\text {stat }}=2(1-\beta) \rho_{1}^{\text {stat }} / \gamma \beta$. Esto nos lleva a

$$
\omega=1-q\left(1-p_{o}\right)\left(1+\frac{1-\beta}{\gamma \beta}\right) \rho_{1}^{\text {stat }} .
$$

En la fase sana $\rho_{1}^{\text {stat }}=0$, por lo tanto $\omega=1.0$ y $\tau=\tau_{0}$. En este caso, la teoría predice que la enfermedad no tiene ningún efecto en el momento del consenso porque no hay nodos infectados que puedan afectar la dinámica de opinión. Sin embargo, tener un valor $\rho_{1}^{\text {stat }}>0$ de nodos infectados en la fase endémica tiene el efecto de reducir $\omega$ o lo que es lo mismo, aumentar $\tau$ con respecto a $\tau_{0}$.

Sustituyendo en la Ec. (3.16) las expresiones para $\gamma$ y $\rho_{1}^{\text {stat }}$ de las Ecs. (3.9) y (3.10), respectivamente, y reordenando algunos términos, obtenemos la siguiente expresión que relaciona $\tau$ y $\tau_{0}$ en la fase endémica:

$$
\frac{\tau}{\tau_{0}} \simeq\left[1-\frac{q\left(1-p_{o}\right)\left\{2(\mu-1)-q\left(1-p_{d}\right)(\mu-2) \beta\right\}\left\{\left[2(2 \mu-1)-q\left(1-p_{d}\right)(\mu-2)\right] \beta-2 \mu\right\}}{\beta\left\{2(\mu-1)-q\left(1-p_{d}\right)(\mu-2)\right\}\left\{\left[2 \mu-q\left(1-p_{d}\right)(\mu-2)\right] \beta-2\right\}}\right]^{-1} .
$$

En la Fig. 3.8 trazamos con líneas continuas la relación $\tau / \tau_{0}$ vs $q$ de la Ec. 3.17) para $\mu=10$. 
Observamos que los valores teóricos de $\tau / \tau_{0}$ son menores que los obtenidos a partir de las simulaciones numéricas (símbolos) para todas las combinaciones de $p_{o}$ y $p_{d}$ que se muestran. Una posible explicación de estas discrepancias se puede dar analizando cómo las correlaciones afectan el número estimado de diferentes tipos de nodos conectados, como lo hemos hecho en la sección 3.3.1 para la prevalencia de la enfermedad. Si tomamos el caso $p_{0}=0$, vemos que un cambio de opinión debido a una interacción entre dos vecinos sociales y de contacto ocurre solo si ambos nodos son susceptibles, es decir, cuando tienen estados $\left[\begin{array}{l}+ \\ 0\end{array}\right]$ y $\left[\begin{array}{c}- \\ 0\end{array}\right]$. Entonces, como la teoría supone que los enlaces sociales +- y los enlaces de contacto 00 no están correlacionados (ver apéndice C), la probabilidad estimada de encontrar un par $\left[\begin{array}{l}+- \\ 0\end{array}\right]$ es mayor que en las simulaciones, dado que se espera que los enlaces sociales +- estén correlacionados negativamente con los enlaces de contacto 00. Esta correlación negativa se debe al hecho de que los vecinos susceptibles tienden a igualar sus opiniones y por lo tanto, es más probable que estén en el mismo estado de opinión en un momento dado. Esto lleva a una sobreestimación del número de pares $\left[\begin{array}{l}+- \\ 0\end{array}\right]$ y por lo tanto, a una mayor tasa de transmisión de opinión. Esto tiene el efecto general de acelerar el consenso, disminuyendo el tiempo medio estimado teóricamente para alcanzar el consenso respecto del tiempo medio de consenso medido en las simulaciones, como vemos en la Fig. 3.8 .

Aunque las discrepancias con los resultados numéricos aumentan con el acoplamiento $q$, la expresión analítica (3.17) puede capturar el comportamiento cualitativo diferente del tiempo de consenso para varias combinaciones de $p_{o}$ y $p_{d}$. Para valores bajos de $p_{d}$, hay una transición a la fase sana cuando $q$ supera un valor $q_{c}<1$ dado por la Ec. (3.12) y por lo tanto, $\tau=\tau_{0}$ para todos $\operatorname{los} q>q_{c}$ (ver las curvas para $p_{d}=0$ en el diagrama principal y el gráfico interno de Fig. 3.8 (a)). Como consecuencia, $\tau / \tau_{0}$ exhibe un comportamiento no monótono con $q$, como describimos en la sección 3.2.2. Para valores más grandes de $p_{d}$, la transición a la fase saludable no ocurre para los valores físicos $q \leq 1$ usados en las simulaciones del modelo, dado que $q_{c}>1$ de la Ec. (3.12). En este caso, $\tau$ puede aumentar monótonamente con $q$ para valores grandes de $p_{d}$ (ver curvas para $p_{d}=1.0$ ), o tener un máximo para valores medios de $p_{d}$ (curvas para $p_{d}=0.5$ ).

Como se explica en la sección 3.2.2, la no monotonía es una consecuencia de la competencia entre el nivel de superposición de enlaces entre las dos redes, que aumenta con $q$, y la prevalencia de la enfermedad, que disminuye con $q$. Esta competencia se puede ver cuantitativamente en la Ec. (3.16) para $\omega$, que tiene tres 
factores que dependen de $q$ y afectan a $\tau$. Además del factor proporcional a $q$, el factor $1 / \gamma$ también aumenta con $q$, como se ve en la Ec. (3.9). Pero estos dos factores se equilibran con $\rho_{1}^{\text {stat }}$, que disminuye con $q$.

Un caso interesante es el de acoplamiento máximo $q=1.0$ y $p_{o}=0$, porque $\tau$ de la Ec. (3.17) diverge a medida que $\beta$ se aproxima a 1.0. Esto sucede en el modelo porque cuando $\beta=1.0$ una vez que un nodo se infecta, permanece infectado para siempre. Luego, una vez que todos los nodos se infectan, la dinámica de opinión se detiene, ya que los nodos vecinos infectados no pueden intercambiar opiniones, y así la capa social se congela en un estado mixto de opiniones $+\mathrm{y}-\mathrm{y}$ nunca se logra el consenso. Al hacer una expansión de la expresión en serie de Taylor (3.17) hasta el segundo orden en un pequeño parámetro $\epsilon=1-\beta \ll 1$ obtenemos, después de un poco de álgebra,

$$
\frac{\tau}{\tau_{0}} \simeq \frac{\left[9-4\left(1-p_{d}\right)\right]^{2}}{90(1-\beta)^{2}},
$$

donde usamos $\mu=10$. La Ec. (3.18) muestra que $\tau$ diverge como $(1-\beta)^{-2}$ en el límite de $\beta \rightarrow 1.0$, como se muestra en el gráfico interno de la Fig. 3.9 (b). Para $\beta=1.0$ y $p_{o}=0$, podemos verificar de la Ec. (3.17) que $\tau / \tau_{0} \simeq 1 /(1-q)$, que muestra la divergencia de $\tau$ a medida que el sistema se aproxima al estado totalmente acoplado $q=1.0$.

\subsection{Discusiones finales}

En este capítulo, propusimos un modelo de red bicapa para explorar la interacción entre la dinámica de formación de opiniones y la propagación de enfermedades en una población de individuos. Utilizamos el Modelo del Votante y el Proceso de Contacto para simular la dinámica de opinión y la de enfermedad que se ejecuta en una red social y de contacto, respectivamente. Estas dos redes comparten los mismos nodos y están unidos por una fracción $q$ de enlaces en común. Mostramos que, cuando las redes están acopladas, la dinámica de opinión puede cambiar drásticamente las propiedades estadísticas de la propagación de la enfermedad, que a su vez modifica las propiedades de la propagación de opiniones, en comparación con el caso de las redes aisladas.

La dinámica del VM puede cambiar el orden de la transición de fase endémicasana observada en el CP cuando la probabilidad de infección $\beta$ excede un valor umbral $\beta_{c}$, desde una transición continua para el caso desacoplado a una transición 
discontinua cuando el acoplamiento $q$ es mayor que cero. La magnitud del salto de la prevalencia de la enfermedad en el punto de transición $\beta_{c}$ aumenta con $q$. La discontinuidad está asociada con la evolución temporal no monótona de la fracción de nodos infectados. Esta no monotonicidad es consecuencia de la naturaleza variable de la probabilidad de infección efectiva, que varía con el tiempo según la evolución estocástica de la fracción de enlaces sociales +- El sistema también muestra una transición discontinua de una fase endémica a una fase sana cuando el acoplamiento supera un valor $q_{c}$, para un valor fijo de $\beta$. El origen de esta discontinuidad es el mismo que el de la transición discontinua con $\beta$, es decir, la no monotonicidad en la evolución temporal de la fracción de nodos infectados. También obtuvimos un diagrama de fases en el espacio $\beta-q$ mostrando las fases sanas y endémicas para diferentes valores de las probabilidades $p_{d}$ y $p_{o}$. En todos los casos, observamos que el punto de transición $\beta_{c}$ aumenta con $q$.

También desarrollamos una aproximación de campo medio que permitió estimar con razonable precisión la línea de transición sana-endémica $\left(\beta_{c}, q_{c}\right)$ en función de los parámetros del modelo. Este enfoque revela que la dinámica de la enfermedad es equivalente a la del CP estándar en una red aislada, pero con una probabilidad de infección efectiva que es constante en el tiempo y que disminuye con el acoplamiento y la fracción estacionaria de enlaces sociales +- para un valor fijo de $\beta$. Esto significa que, a nivel de campo medio, el efecto general del VM sobre el CP es disminuir la probabilidad de infección efectiva a medida que aumenta el acoplamiento. Por lo tanto, cuando $q$ aumenta, se necesita un valor mayor de $\beta$ para llevar el sistema a la fase endémica, lo que lleva a un aumento del punto de transición $\beta_{c}$ con $q$.

Por su parte, la dinámica de CP tiene el efecto general de ralentizar la propagación de opiniones, retrasando el proceso de consenso de opinión en comparación con el observado en una red aislada. La aproximación de MF revela que la dinámica de opinión corresponde a la del VM estándar en una red aislada, pero con una probabilidad de transmisión de opinión que disminuye con $q$ y la prevalencia de la enfermedad. Dependiendo de los valores de los parámetros, el tiempo medio de consenso $\tau$ puede mostrar un aumento monótono con $q$, así como un comportamiento no monótono. Este enfoque teórico dió una idea de estos resultados, lo que permitió obtener una expresión matemática aproximada que relaciona a $\tau$ con los parámetros. Esta aproximación muestra que el comportamiento de $\tau$ con $q$ es el resultado de dos mecanismos diferentes en juego: la superposición de enlaces sociales y de 
contacto que tiende a aumentar $\tau$ con $q$, que se compensa con la fracción de nodos infectados que tiende a disminuir $\tau$ con $q$. Por lo tanto, la dependencia no trivial de $\tau$ con $q$ es una consecuencia de la competencia entre estos dos mecanismos.

Es interesante observar que, a pesar de la interacción no trivial entre el CP y el VM, el sistema interdependiente de opiniones y enfermedad puede verse aproximadamente como dos sistemas que evolucionan independientemente el uno del otro, donde cada sistema tiene un parámetro efectivo que depende de la otra dinámica y el acoplamiento. Específicamente, la dinámica de opinión corresponde a la del VM con una probabilidad de transmisión de opinión efectiva que disminuye con la prevalencia de la enfermedad y el acoplamiento, mientras que la propagación de la enfermedad está bien descrita por la dinámica del CP con una probabilidad de infección efectiva que disminuye con la fracción de enlaces sociales +- y el acoplamiento. Sin embargo, esta es solo una aproximación que proviene del análisis de MF, que desprecia las correlaciones entre los estados de opinión y enfermedad. 



\section{Capítulo 4}

\section{Propagación de Enfermedades e Infor- mación en Redes Múltiples}

Los estudios matemáticos y computacionales de modelos epidémicos han demostrado ser muy importantes para comprender la dinámica de las enfermedades en el mundo real [78]. La principal motivación detrás del modelado de epidemias es el desarrollo de métodos para controlar la transmisión de enfermedades aumentando nuestra comprensión sobre las mismas [78,98]. Las estrategias más tradicionales para contener un brote se basan en la vacunación [98 o la cuarentena [99]. Sin embargo, trabajos recientes verificaron que la concientización frente a enfermedades infecciosas también puede interferir en la propagación de las mismas [100].

Los métodos de prevención individuales pueden reducir considerablemente el alcance general de una epidemia, como se mostró en estudios previos [48, 49, 101]. En estos trabajos se ha analizado el impacto de la información sobre la propagación de epidemias en una población $48,50,101,103$. Una forma de modelar la influencia del comportamiento humano en la propagación de una epidemia es utilizar un modelo de propagación de rumores para simular la difusión del conocimiento sobre la enfermedad (y sus métodos de prevención) de boca en boca. En este marco, los rumores -que en lo sucesivo llamaremos información- y las epidemias, consisten en dos procesos de difusión que interactúan entre sí.

Esta interacción entre individuos puede representarse en una red compleja múltiple, donde la enfermedad y el conocimiento de la información para prevenir la transmisión se propagan en diferentes capas. La capa de la enfermedad puede representar contactos físicos o de proximidad para la propagación de enfermedades aerotransportadas en personas que interactúan regularmente (familia, compañeros de trabajo,...) u ocasionalmente (personas que comparten transporte público). La 
capa de información representa contactos entre personas que intercambian información personalmente o de manera virtual: Redes Sociales. Granell y colaboradores verificaron que la transmisión de una enfermedad se reduce de acuerdo a la interacción entre estas dos capas 48,49. En la vida real, se espera que estas dinámicas no evolucionan a la misma velocidad, por este motivo se debe escoger una escala de tiempo ajustable que promueva una alternancia aleatoria entre los procesos epidémicos y los procesos de información. En este capítulo exploramos la interacción de ambos procesos agregando diferentes escalas temporales para cada uno de ellos.

\subsection{Descripción del Modelo SIS-UAU}

El modelo empleado involucra las dinámicas SIS-UAU, similar al trabajo propuesto por Granell y colaboradores [48,49]. Se plantea el uso del SIS para ambos procesos -epidémico e informativo-, donde cada uno está representado por una capa de la red como se observa en la Fig. 4.1. En la capa epidémica, los nodos pueden estar en estado Susceptible (S) o Infectado (I) donde cada nodo infectado de la red trata de tranmitirle la enfermedad a cada uno de sus vecinos susceptibles con tasa $\beta$ y luego con tasa $\mu$ se recupera.

En la capa de información el modelo funciona de manera similar, cambiando los nombres de los estados a No informado (U) e Informado (A) para evitar confusiones con el proceso epidémico, las letras U y A provienen del inglés Unaware y Aware, respectivamente. Un nodo no informado puede informarse con tasa $\gamma$ al contactarse con un vecino informado, y, de manera análoga, un nodo informado puede "olvidar" la información (o simplemente perder el interés en ella) y volver a ser no informado con tasa $\alpha$.

Como se mencionó anteriormente, el modelo SIS básico se modifica para introducir la interacción entre la información y las epidemias. La información se considera como el conocimiento de los métodos de prevención para que las personas informadas reduzcan su probabilidad de contraer la enfermedad. Esto se modela como una reducción en la tasa de contagio de un nodo informado por un factor de $\Gamma(0 \leq \Gamma \leq 1)$. Entonces, un nodo informado tendrá una tasa reducida $\Gamma \beta$ de contraer la enfermedad. 


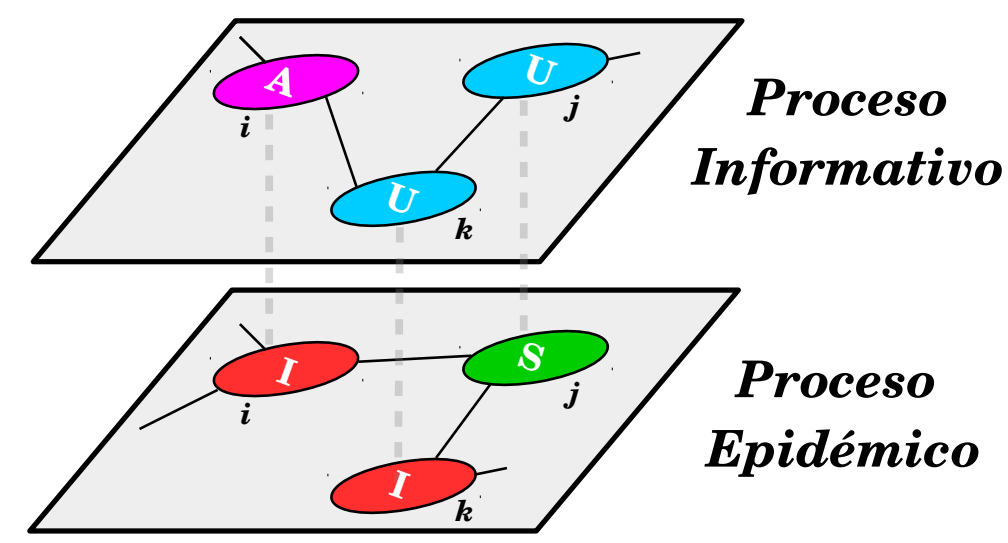

Figura 4.1: Esquema de un pequeño trozo de la estructura multiplex utilizada para el modelo SIS-UAU. En la capa superior (Proceso Informativo) los nodos tienen dos estados posibles: no informado (U) e informado (A). En la capa inferior (Proceso Epidémico) los nodos son los mismos individuos que en la capa superior y sus estados puede ser: susceptible (S) o infectado (I).

Por otro lado, la existencia de nodos infectados refuerza la información sobre la enfermedad, esto se incluye en el modelo como una "autoconciencia" de las personas infectadas donde los nodos infectados y no informados tienen una tasa $\kappa$ de informarse. Representamos el estado compuesto de un nodo con dos letras mayúsculas, la primera para el estado de epidemia y la segunda para el estado de información: susceptible-no informado SU, susceptible-informado SA, infectado-no informado IU, e infectado-informado IA.

Esquemáticamente, dividiremos cada transición de estado del modelo en dos grupos: epidémico e informativo. La transmisión de la enfermedad será: $\mathrm{SU} \stackrel{\beta}{\longrightarrow} \mathrm{IU}$, $\mathrm{SA} \stackrel{\Gamma \beta}{\longrightarrow}$ IA y la recuperación $\mathrm{I} \stackrel{\mu}{\longrightarrow} \mathrm{S}$, también es considerada en este grupo de transiciones. Mientras que la transmisión de la información $\mathrm{U} \stackrel{\gamma}{\longrightarrow} \mathrm{A}$, la pérdida de la información $\mathrm{A} \stackrel{\alpha}{\longrightarrow} \mathrm{U}$ y la autoconciencia (IU $\stackrel{\kappa}{\longrightarrow}$ IA) se agrupan como transiciones de información.

Como se mencionó anteriormente, se espera que las dinámicas descritas no evolucionen a la misma velocidad y por este motivo se debe escoger una escala de tiempo ajustable entre ambos procesos. Esta escala de tiempo promueve una alternancia aleatoria entre los procesos epidémicos y los procesos de información durante toda la dinámica donde el parámetro $\pi$ aumenta la velocidad de propagación de la información. Todas las transiciones de este modelo están esquematizadas 


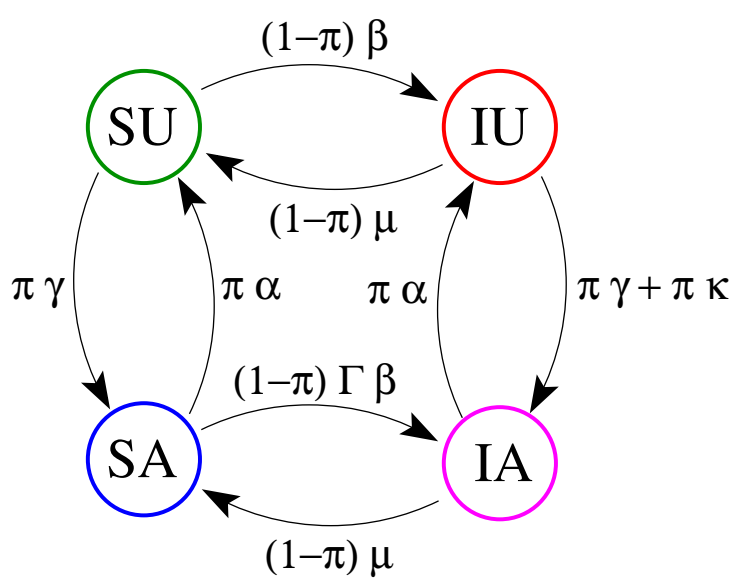

Figura 4.2: Transiciones de la dinámica para ambos grupos: epidémico e informativo.

en la Fig. 4.2

\subsection{Aproximación de campo medio}

Ahora estudiamos el comportamiento del modelo SIS/UAU utilizando una aproximación de campo medio que asume que, en cada paso de la dinámica, cada nodo interactúa con $\eta$ vecinos elegidos al azar. Esta aproximación no toma en cuenta las correlaciones de estado del vecino más cercano y debería funcionar razonablemente bien para redes aleatorias con distribuciones de grado homogéneas y sin correlaciones de grado, como las redes Erdös Rényi.

Las densidades de los nodos en cada estado $\rho_{i u}, \rho_{s u}, \rho_{i a}$ y $\rho_{s a}$ evolucionan de acuerdo con el siguiente conjunto de ecuaciones acopladas:

$$
\begin{aligned}
\frac{d \rho_{i u}}{d t} & =(1-\pi) \beta \eta \rho_{s u} \rho_{i}+\pi \alpha \rho_{i a}-(1-\pi) \mu \rho_{i u}-\pi \kappa \rho_{i u}-\pi \gamma \eta \rho_{i u} \rho_{a} \\
\frac{d \rho_{s u}}{d t} & =(1-\pi) \mu \rho_{i u}+\pi \alpha \rho_{s a}-(1-\pi) \beta \eta \rho_{s u} \rho_{i}-\pi \gamma \eta \rho_{s u} \rho_{a} \\
\frac{d \rho_{i a}}{d t} & =\pi \gamma \eta \rho_{i u} \rho_{a}+\pi \kappa \rho_{i u}+(1-\pi) \Gamma \beta \eta \rho_{s a} \rho_{i}-\pi \alpha \rho_{i a}-(1-\pi) \mu \rho_{i a} \\
\frac{d \rho_{s a}}{d t} & =\pi \gamma \eta \rho_{s u} \rho_{a}+(1-\pi) \mu \rho_{i a}-\pi \alpha \rho_{s a}-(1-\pi) \Gamma \beta \eta \rho_{s a} \rho_{i},
\end{aligned}
$$

donde $\eta$ es el grado medio de la red, $\beta, \mu, \gamma, \Gamma, \kappa, \alpha$ son tasas de transición y $\rho_{i}=\rho_{i u}+\rho_{i a}$ y $\rho_{a}=\rho_{i a}+\rho_{s a}$ son las densidades de nodos infectados e informados, respectivamente. Además, la relación de conservación del número total de nodos $\rho_{i u}+\rho_{s u}+\rho_{i a}+\rho_{s a}=1$ permanece constante en todo momento. Los términos de 
ganancia y pérdida en las Ecs. 4.1)corresponden a las flechas entrantes y salientes de la Fig. 4.2. Las Ecs. (4.1) describen, en el límite de tiempo continuo, la dinámica SIS/UAU, donde las probabilidades de infección y recuperación de la enfermad en un paso de tiempo infinitesimal $d t$ son $\beta d t$ y $\mu d t$, respectivamente, y de manera análoga para los eventos de transmisión de información.

\subsubsection{Solución para $\gamma=0, \Gamma=0 \mathrm{y} \kappa=1.0$}

Las Ecs. (4.1) se simplifican cuando establecemos $\gamma=0, \Gamma=0$ y $\kappa=1.0, \mathrm{y}$ esto corresponde a los parámetros utilizados en la Fig. 4.5 (arriba-derecha) de la sección 4.3. Las ecuaciones simplificadas resultan

$$
\begin{aligned}
\frac{d \rho_{i u}}{d t} & =(1-\pi) \beta \eta \rho_{s u} \rho_{i}+\pi \alpha \rho_{i a}-(1-\pi) \mu \rho_{i u}-\pi \rho_{i u} \\
\frac{d \rho_{s u}}{d t} & =(1-\pi) \mu \rho_{i u}+\pi \alpha \rho_{s a}-(1-\pi) \beta \eta \rho_{s u} \rho_{i} \\
\frac{d \rho_{i a}}{d t} & =\pi \rho_{i u}-\pi \alpha \rho_{i a}-(1-\pi) \mu \rho_{i a} \\
\frac{d \rho_{s a}}{d t} & =(1-\pi) \mu \rho_{i a}-\pi \alpha \rho_{s a} .
\end{aligned}
$$

El punto fijo trivial de este sistema de ecuaciones es $\rho_{s u}=1.0$, y corresponde a una población totalmente sana y no informada. El punto fijo no trivial corresponde a las densidades estacionarias

$$
\begin{aligned}
\rho_{i u}^{*} & =\frac{\alpha(\beta \eta-\mu)}{\beta \eta(1+\alpha)} \\
\rho_{i a}^{*} & =\frac{\pi \alpha(\beta \eta-\mu)}{\beta \eta(1+\alpha)[\pi \alpha+(1-\pi) \mu]} \\
\rho_{s u}^{*} & =\frac{\mu}{\beta \eta} \\
\rho_{s a}^{*} & =\frac{(1-\pi) \mu(\beta \eta-\mu)}{\beta \eta(1+\alpha)[\pi \alpha+(1-\pi) \mu]} .
\end{aligned}
$$

La expresión para la prevalencia de la enfermedad resulta

$$
\rho_{i}^{*}=\frac{\alpha(\beta \eta-\mu)[\pi(1+\alpha)+(1-\pi) \mu]}{\beta \eta(1+\alpha)[\pi \alpha+(1-\pi) \mu]}
$$

y es graficada en la Fig. 4.3 para los valores de los parámetros $\mu=0.9, \alpha=0.6$, $\beta=0.3$ y $\eta=6$. Observamos que $\rho_{i}^{*}$ aumenta con $\pi$ para todo $\kappa>0$.

La Ec. 4.7) predice que la prevalencia toma el valor $\rho_{i}^{*}=\alpha(\beta \eta-\mu) /[(1+$ $\alpha) \beta \eta]=0.1875$ y $\rho_{i}^{*}=(\beta \eta-\mu) / \beta \eta=0.5$ en los límites $\pi=0$ y $\pi=1$, respectivamente. Sin embargo, estos casos extremos pueden ser engañosos ya que los valores 


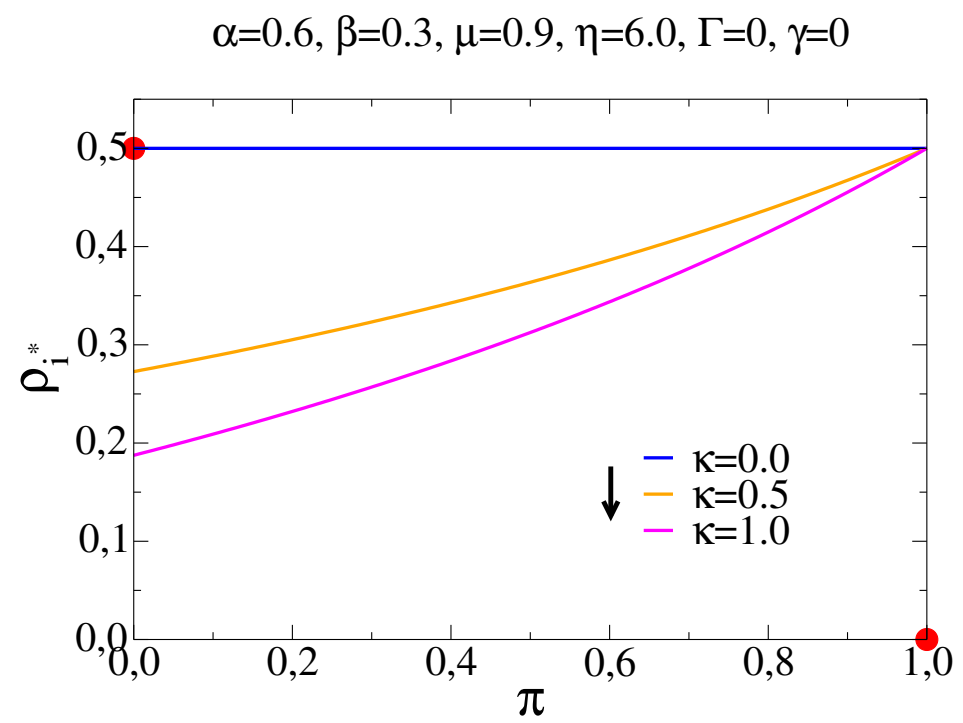

Figura 4.3: Prevalencia de la enfermedad como función de $\pi$.

cercanos a esos límites no corresponden al valor para $\rho_{i}^{*}$ en esos puntos. Esto quiere decir que $\rho_{i}^{*}$ presenta una discontinuidad en $\pi=0$ y $\pi=1$. Para observar esto, reescribiremos las Ecs. (4.2) para $\pi=0$

$$
\begin{aligned}
\frac{d \rho_{i u}}{d t} & =\beta \eta \rho_{s u} \rho_{i}-\mu \rho_{i u} \\
\frac{d \rho_{s u}}{d t} & =\mu \rho_{i u}-\beta \eta \rho_{s u} \rho_{i} \\
\frac{d \rho_{i a}}{d t} & =-\mu \rho_{i a} \\
\frac{d \rho_{s a}}{d t} & =\mu \rho_{i a},
\end{aligned}
$$

cuya solución estacionaria no trivial es $\rho_{i u}=C_{0}-\mu / \beta \eta, \rho_{i a}=0, \rho_{s u}=\mu / \beta \eta \mathrm{y}$ $\rho_{s a}=1-C_{0}$, donde $C_{0}=\rho_{u}(t=0)$ es una constante. Asumiendo que todos los individuos son no informados inicialmente, $C_{0}=1$, lo que conduce a una prevalencia $\rho_{i}^{*}=(\beta \eta-\mu) / \beta \eta=0.5$ en $\pi=0$, que es más alto por un factor $(1+\alpha) / \alpha=2.66$ que el límite $\pi \rightarrow 0$ de la Ec. (4.7), mostrando la discontinuidad en $\pi=0$ (ver Fig. 4.3). Para $\pi=1$ las Ecs. 4.2 se reducen a

$$
\begin{aligned}
\frac{d \rho_{i u}}{d t} & =\alpha \rho_{i a}-\rho_{i u} \\
\frac{d \rho_{s u}}{d t} & =\alpha \rho_{s a} \\
\frac{d \rho_{i a}}{d t} & =\rho_{i u}-\alpha \rho_{i a} \\
\frac{d \rho_{s a}}{d t} & =-\alpha \rho_{s a},
\end{aligned}
$$


cuya solución estacionaria es $\rho_{i u}=\alpha C_{1} /(1+\alpha), \rho_{i a}=C_{1} /(1+\alpha), \rho_{s u}=1-C_{1} \mathrm{y}$ $\rho_{s a}=0$, donde $C_{1}=\rho_{i}(t=0)$. Esto significa que la fracción de nodos infectados se mantiene constante en el tiempo: $\rho_{i}=C_{1}$. Si inicialmente hay un individuo infectado, entonces la prevalencia es $\rho_{i}^{*}=1 / N \ll 1$ para valores grandes de $N$. Esto muestra el salto de $\rho_{i}^{*}$ desde 0.5 hasta un valor cercano a cero para $\pi=1$ (Fig. 4.3).

Observamos que la densidad estacionaria de los nodos informados $\rho_{a}=(\beta \eta-$ $\mu) /[\beta \eta(1+\alpha)]$ es independiente de $\pi$, mientras que $\rho_{i}^{*}$ depende de $\pi$. Esto significa que ambas dinámicas SIS y UAU son cíclicas pero no equivalentes. La equivalencia se rompe gracias al término $\kappa \pi$ en la transición espontánea $I U \rightarrow I A$. De hecho, para el caso $\kappa=0$, se obtiene $\rho_{i}^{*}=(\beta \eta-\mu) / \beta \eta$ independiente de $\pi$ (ver Fig. 4.3).

\subsubsection{Solución para $\gamma=0$}

Para $\gamma=0$, las Ecs. (4.1) se convierten en

$$
\begin{aligned}
\frac{d \rho_{i u}}{d t} & =(1-\pi) \beta \eta \rho_{s u} \rho_{i}+\pi \alpha \rho_{i a}-(1-\pi) \mu \rho_{i u}-\pi \kappa \rho_{i u}, \\
\frac{d \rho_{s u}}{d t} & =(1-\pi) \mu \rho_{i u}+\pi \alpha \rho_{s a}-(1-\pi) \beta \eta \rho_{s u} \rho_{i}, \\
\frac{d \rho_{i a}}{d t} & =\pi \kappa \rho_{i u}+(1-\pi) \Gamma \beta \eta \rho_{s a} \rho_{i}-\pi \alpha \rho_{i a}-(1-\pi) \mu \rho_{i a}, \\
\frac{d \rho_{s a}}{d t} & =(1-\pi) \mu \rho_{i a}-\pi \alpha \rho_{s a}-(1-\pi) \Gamma \beta \eta \rho_{s a} \rho_{i} .
\end{aligned}
$$

Las soluciones estacionarias de las Ecs. (4.1) pueden ser obtenidas estudiando el caso $\gamma=0$.

Las densidades estacionarias triviales del sistema de Ecs. 4.10 son $\rho_{s u}^{*}=1$ $\mathrm{y} \rho_{i u}^{*}=\rho_{i a}^{*}=\rho_{s a}^{*}=0$. Para encontrar las densidades estacionarias no triviales comenzamos igualando todas las derivadas a cero, sumamos las Ecs. 4.10a y 4.10c y luego se obtiene la siguiente relación entre $\rho_{s u}^{*} \mathrm{y} \rho_{s a}^{*}$ :

$$
\beta \eta\left(\rho_{s u}^{*}+\Gamma \rho_{s a}^{*}\right)-\mu=0,
$$


para $\rho_{i}^{*} \neq 0$. Luego, resolvemos para $\rho_{i u}^{*}$ y $\rho_{i a}^{*}$ de las Ecs. 4.10b y $4.10 \mathrm{~d}$, respectivamente,

$$
\begin{aligned}
\rho_{i u}^{*} & =\frac{(1-\pi) \beta \eta \rho_{s u}^{*} \rho_{i}^{*}-\pi \alpha \rho_{s a}^{*}}{(1-\pi) \mu}, \\
\rho_{i a}^{*} & =\frac{(1-\pi) \Gamma \beta \eta \rho_{s a}^{*} \rho_{i}^{*}+\pi \alpha \rho_{s a}^{*}}{(1-\pi) \mu} .
\end{aligned}
$$

Sustituyendo estas expresiones en la Ec. 4.10c llegamos, luego de hacer un poco de álgebra, a la siguiente ecuación que relaciona $\rho_{s u}^{*}$ y $\rho_{s a}^{*}$ :

$$
(1-\pi) \beta \eta\left(1-\rho_{s u}^{*}-\rho_{s a}^{*}\right)\left(\kappa \rho_{s u}^{*}-\alpha \Gamma \rho_{s a}^{*}\right)-[\pi(\kappa+\alpha)+(1-\pi) \mu] \alpha \rho_{s a}^{*}=0 .
$$

Insertando la expresión

$$
\rho_{s u}^{*}=\frac{\mu}{\beta \eta}-\Gamma \rho_{s a}^{*}
$$

de la Ec. (4.11) en la Ec. 4.14 y expandiendo en potencias de $\rho_{s a}^{*}$ obtenemos la ecuación cuadrática

$$
a \rho_{s a}^{* 2}+b \rho_{s a}^{*}+c=0
$$

con coeficientes

$$
\begin{aligned}
a & =(1-\pi) \beta^{2} \eta^{2} \Gamma(1-\Gamma)(\kappa+\alpha) \\
b & =-\beta \eta\{(\kappa+\alpha)[\pi \alpha+(1-\pi) \mu]+(1-\pi) \Gamma[(\kappa+\alpha)(\beta \eta-\mu)-\mu \kappa]\}, \\
c & =(1-\pi)(\beta \eta-\mu) \kappa \mu .
\end{aligned}
$$

La solución física $0 \leq \rho_{s a}^{*} \leq 1$ de la Ec. (4.16 viene dada por la expresión

$$
\rho_{s a}^{*}=\frac{-b-\sqrt{b^{2}-4 a c}}{2 a}
$$

Una expresión cerrada para $\rho_{s u}^{*}$ puede ser obtenida directamente insertando la expresión de la Ec. (4.18) en la Ec. (4.15). Para obtener expresiones cerradas para $\rho_{i u}^{*}$ y $\rho_{i a}^{*}$ podemos usar primero la equivalencia

$$
\rho_{i}^{*}=1-\rho_{s u}^{*}-\rho_{s a}^{*}
$$

en la Ec. 4.12 y la Ec. 4.13, respectivamente, y luego insertar las expresiones para $\rho_{s a}^{*}$ y $\rho_{s u}^{*}$ dentro de las expresiones resultantes. Finalmente, podemos obtener una expresión para la prevalencia usando la Ec. (4.19) y la Ec. 4.15), reescribiendo $\rho_{i}^{*}$ in términos de $\rho_{s a}^{*}$

$$
\rho_{i}^{*}=\frac{\beta \eta-\mu}{\beta \eta}-(1-\Gamma) \rho_{s a}^{*}
$$


que se reduce a la expresión citada en la Ec. 4.22 de la sección 4.2 .2 después de reemplazar la expresión de la Ec. 4.18 para $\rho_{s a}^{*}$.

Finalmente, las densidades estacionarias resultan:

$$
\begin{aligned}
& \rho_{s a}^{*}=\frac{-b-\sqrt{b^{2}-4 a c}}{2 a}, \\
& \rho_{s u}^{*}=\frac{\mu}{\beta \eta}-\Gamma \rho_{s a}^{*}, \\
& \rho_{i a}^{*}=\frac{(1-\pi) \Gamma \beta \eta \rho_{s a}^{*}\left(1-\rho_{s a}^{*}-\rho_{s u}^{*}\right)+\pi \alpha \rho_{s a}^{*}}{(1-\pi) \mu} \\
& \rho_{i u}^{*}=\frac{(1-\pi) \beta \eta \rho_{s u}^{*}\left(1-\rho_{s a}^{*}-\rho_{s u}^{*}\right)-\pi \alpha \rho_{s a}^{*}}{(1-\pi) \mu} .
\end{aligned}
$$

La densidad estacionaria de nodos infectados $\rho_{i}^{*}=\rho_{i u}^{*}+\rho_{i a}^{*}$ en la fase endémicainformada, sustituyendo la expresión para $\rho_{s a}^{*}$ se puede escribir como

$$
\rho_{i}^{*}=\frac{\beta \eta-\mu}{\beta \eta}+\frac{(1-\Gamma)\left(b+\sqrt{b^{2}-4 a c}\right)}{2 a} .
$$

La expresión para la prevalencia de la enfermedad $\rho_{i}^{*}$ como función de $\pi$ usando la Ec. (4.22 se grafica en la Fig 4.4 donde observamos que la dependencia de la enfermedad con $\pi$ se reduce a medida que $\Gamma$ aumenta y desaparece para $\Gamma=1.0$ donde se recupera la dinámica SIS estándar y, por lo tanto, $\rho_{i}^{*}$ es independiente de $\pi$.

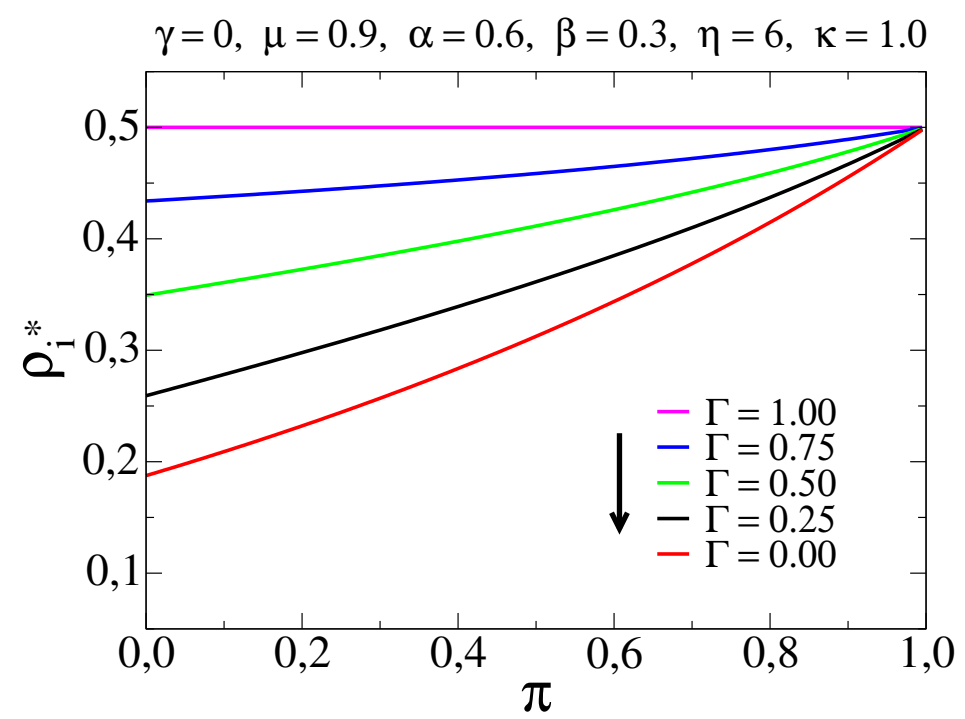

Figura 4.4: Prevalencia de la enfermedad $\rho_{i}^{*}$ como función de $\pi$ (Ec. 4.22) variando $\Gamma$. Para $\Gamma=1.0$ se recupera la dinámica SIS estándar. 


\subsection{Análisis numérico de las ecuaciones}

Consideramos una red múltiple con dos capas independientes que representan la propagación de una enfermedad y la transmisión de información sobre el conocimiento de dicha enfermedad. Presentamos la solución de las Ecs. (4.1) propuestas en la sección anterior 4.2 mediante integración numérica, incluyendo el caso $\gamma>0$, el cual no se tuvo en cuenta en los resultados analíticos.

Utilizando los mismos valores para los parámetros de la Fig $4.4, \beta=0.3$, $\mu=0.9, \alpha=0.6$ y $\eta=6.0$, en la Fig 4.5 graficamos la prevalencia de la enfermedad como función de la velocidad de propagación $\pi$ para varios conjuntos de parámetros. Se muestran 3 valores para la tasa de que los agentes se informen $\gamma=0.0,0.1 \mathrm{y}$ 0.3. Los dos gráficos superiores de esta Figura son para $\Gamma=0$ y presentan un aumento de la densidad de infectados $\rho_{i}^{*}$ con $\pi$ en un rango similar para 2 valores del parámetro $\kappa$, el cual aumenta la tasa de que los agentes en estado infectado se informen. Para $\gamma=0.3$ la densidad de infectados desaparece $\rho_{i}^{*}=0$, cuando esto ocurre la densidad de agentes informados es $\rho_{a}^{*}=\rho_{s a}^{*}=(\eta \gamma-\alpha) / \eta \gamma=2 / 3$ (despejando de la Ec.(4.1b)) y la densidad restante de agentes es $\rho_{s u}^{*}$. Para los gráficos inferiores con $\Gamma=0.5$, observamos que el aumento de la prevalencia con $\pi$ es más lento y $\rho_{i}^{*} \neq 0$ para los mismos valores de $\gamma$.

En todos los paneles de la Fig. 4.5 la prevalencia de la enfermedad aumenta con la velocidad de propagación de la información $\pi$, este es un resultado contrario a lo esperado y es, probablemente, el más interesante de todo el análisis de este capítulo ya que si hay más información disponible para la población, se esperaría que la densidad de enfermos disminuya y esto no ocurre en los resultados mostrados. Este hecho representa un problema abierto de este trabajo en desarrollo.

Continuando el análisis, en la Fig. 4.6. se muestra la densidad de agentes infecta$\operatorname{dos} \rho_{i}^{*}$ (panel (a)) e informados $\rho_{a}^{*}$ (panel (b)) como función de $\pi$ para $\Gamma=\kappa=0.5$. Cuando $\pi$ aumenta disminuyen los agentes informados, lo que también resulta contradictorio.

En la Fig. 4.7 se muestra la densidad de individuos infectados $\rho_{i}^{*}$ en función de la tasa de infección $\beta$ para $\gamma=0.1$ y 3 valores de $\pi=0.1,0.5$ y 0.9. En los gráficos superiores tomamos $\Gamma=0.0$ y 2 valores de $\kappa=0.5$ y 1.0. Las curvas de $\rho_{i}^{*}$ aumentan con $\pi$, lo cual coincide con los resultados de la Fig.4.4. Para $\Gamma=0.5$ (gráficos inferiores), las curvas de $\rho_{i}$ también aumentan con $\pi$ en un rango menor. 

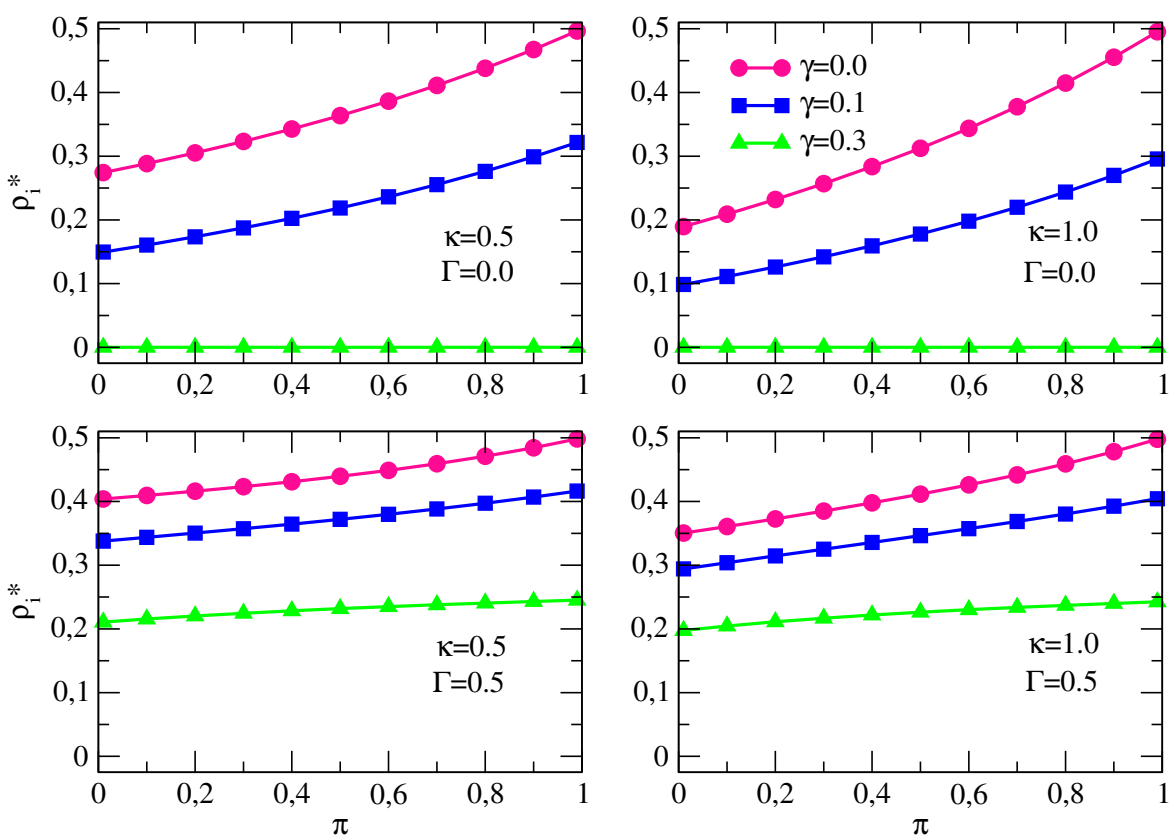

Figura 4.5: Densidad estacionaria de nodos infectados como función del parámetro temporal $\pi$ con $\gamma=0.0,0.1$ y 0.3 , para diferentes valores de $\Gamma$ y $\kappa$. El valor de los otros parámetros utilizados es: $\beta=0.3, \mu=0.9, \alpha=0.6$ у $\eta=6.0$.
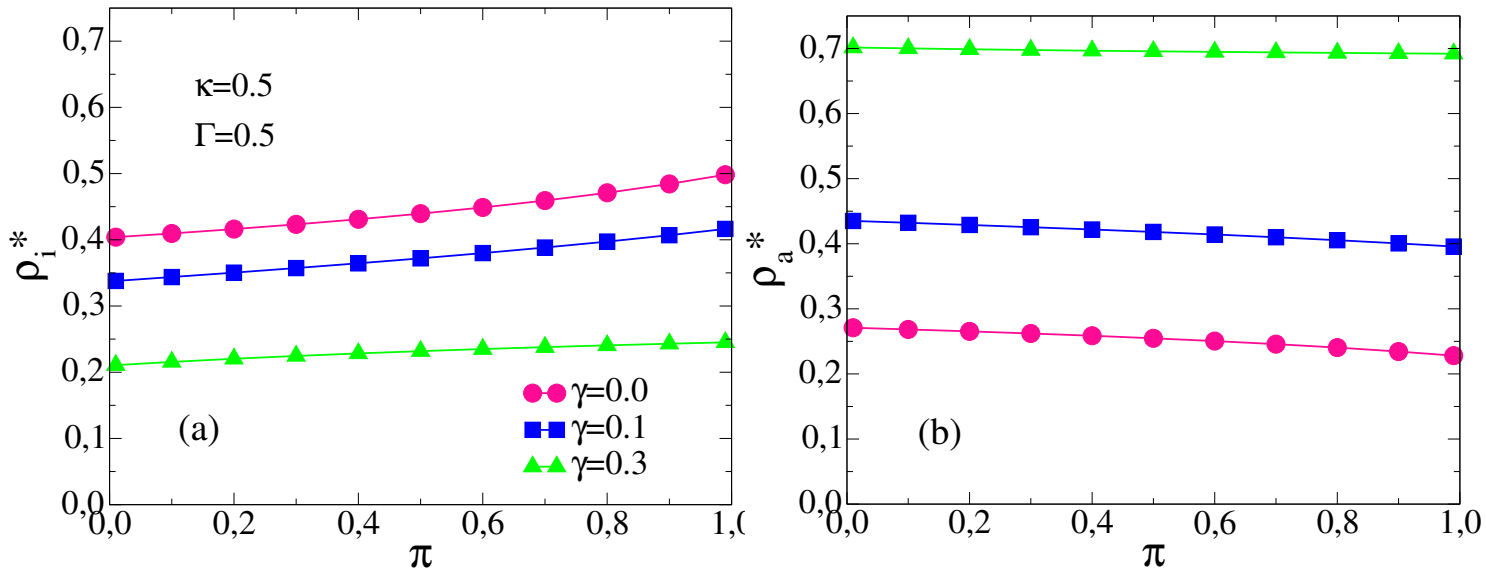

Figura 4.6: Densidad estacionaria de nodos infectados (panel (a)) e informados (panel (b)) como función de la velocidad de propagación de la información $\pi$ para 3 valores de $\gamma=0.0,0.1,0.3$ y para $\Gamma=\kappa=0.5$. A medida que $\pi$ aumenta también lo hacen los individuos infectados mientras que disminuyen los individuos informados, esto representa un comportamiento contrario a lo esperado. 

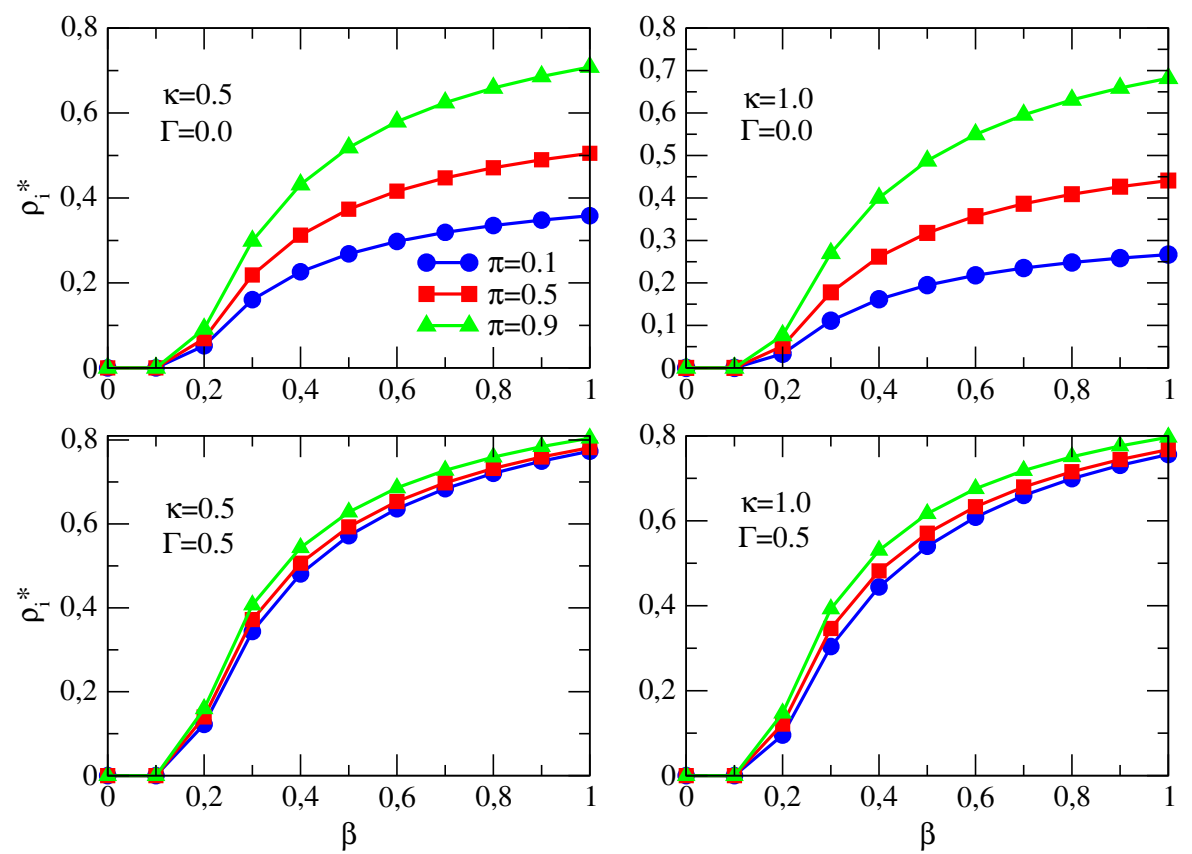

Figura 4.7: Densidad estacionaria de nodos infectados como función de la tasa de infección $\beta$ con $\gamma=0.1$, para diferentes valores de la velocidad de propagación $\pi$. El valor de los otros parámetros utilizados es: $\mu=0.9, \alpha=0.6$ y $\eta=6.0$.

El parámetro $\Gamma$ regula la infección en los agentes informados, cuya densidad $\left(\rho_{i a}\right)$ disminuye conforme $\Gamma$ disminuye.

La construcción de este modelo involucra muchos parámetros asociados a cada proceso de transición. Cada uno de estos procesos puede ser analizado siguiendo el diagrama de transiciones de la Fig.4.2. Si nos centramos en la prevalencia de la enfermedad $\rho_{i}^{*}$, podemos describir su comportamiento esperado respecto de cada parámetro y esto lo resumimos a continuación en el siguiente esquema en términos de aumento $\uparrow$ y disminución $\downarrow$ de cada cantidad:

$$
\begin{aligned}
\mu \uparrow, \gamma \uparrow, \kappa \uparrow & \Longrightarrow \rho_{i}^{*} \downarrow \\
\Gamma \uparrow \beta \uparrow, \alpha \uparrow, \pi \uparrow & \Longrightarrow \rho_{i}^{*} \uparrow
\end{aligned}
$$

Salvo por el comportamiento de $\rho_{i}^{*}$ con $\pi$, este esquema concuerda con los resultados presentados en esta sección donde describimos el comportamiento de las Ecs.4.1. En la Fig. 4.8 mostramos el diagrama de fases $\beta-\gamma$ donde queda contenido todo nuestro análisis para $\pi=0.5$. La información, representada por el parámetro $\gamma$, reduce la cantidad de infectados, y por ende la transición desde una fase sana a una fase endémica, lo cual se hace más notorio al variar el parámetro $\Gamma$. 
En la Fig. 4.9 incluímos el parámetro $\Gamma$ para mostrar el diagrama de fases de la Fig. 4.8 en 3 dimensiones. Al incorporar $\Gamma$ podemos observar como se curva el plano que separa las fases sana y endémica más claramente.

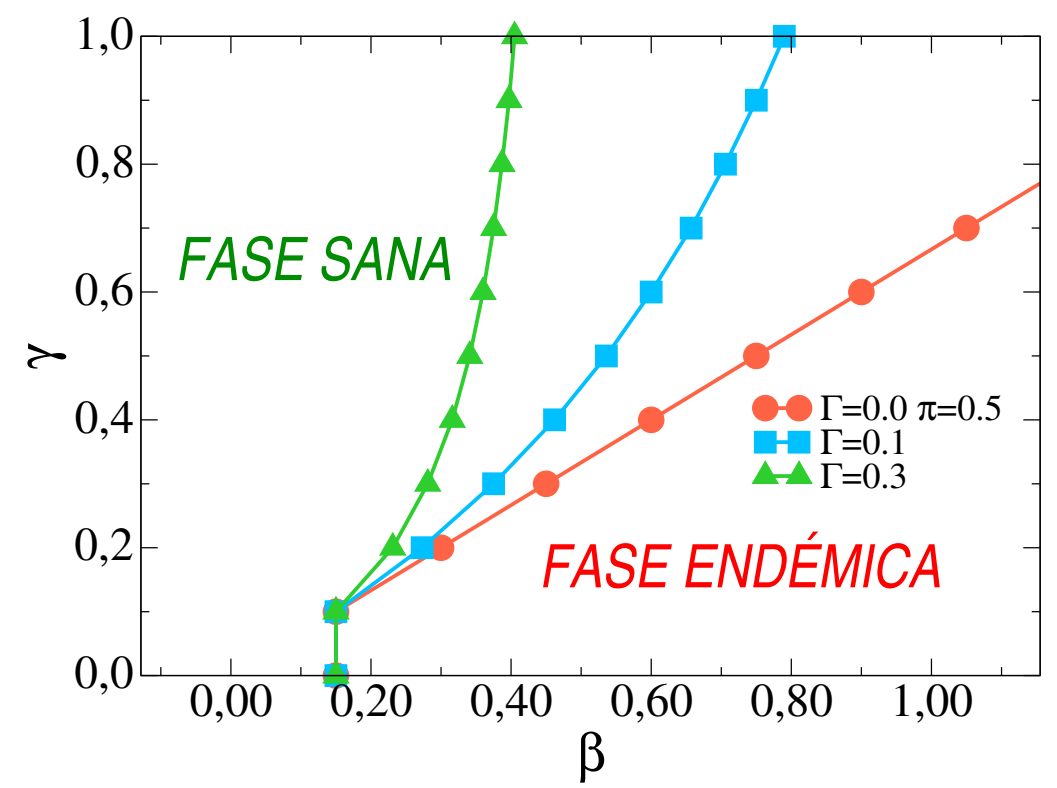

Figura 4.8: Diagrama de fase $\beta-\gamma$ para 3 valores de $\Gamma$ con $\pi=0.5$.

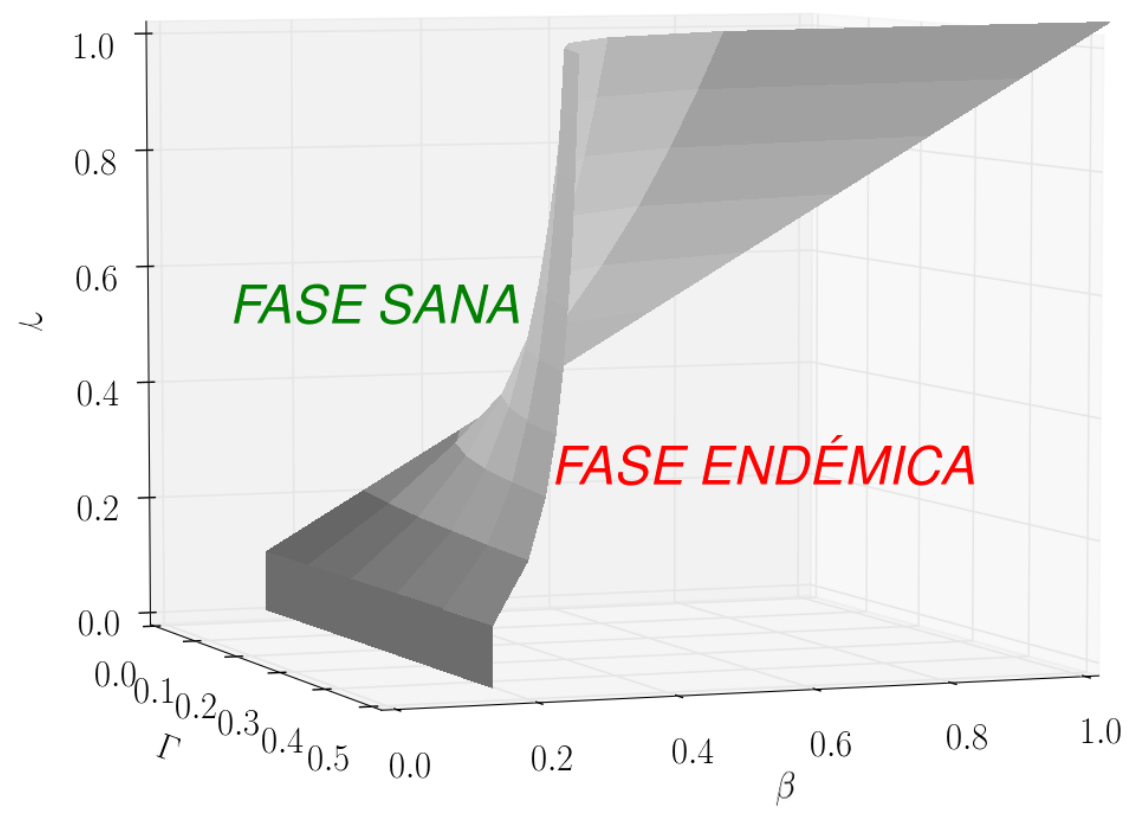

Figura 4.9: Diagrama de fase $\beta-\gamma-\Gamma$. 


\subsection{Discusiones preliminares}

En este capítulo, presentamos la aproximación de campo medio y el análisis de las ecuaciones con escala de tiempo variable que describe un modelo de red bicapa que representa la propagación de una enfermedad y la transmisión de información sobre el conocimiento de dicha enfermedad. La construcción de este modelo involucra un gran número de parámetros para su caracterización, cuya pregunta fundamental se centra en mostrar cuan beneficiosa resulta la información disponible sobre una enfermedad para reducir el brote de la misma. El parámetro $\gamma$ representa la tasa de informarse mientras que el parámetro $\pi$ es la velocidad de propagación de la información. Si bien, cuando $\gamma$ aumenta se reduce la cantidad de infectados, al variar $\pi$ nos encontramos con un hecho contradictorio: el aumento en la velocidad de propagación de la información aumenta la cantidad de individuos infectados y disminuye la cantidad de individuos informados, algo paradójicamente inesperado. Este hecho representa un problema abierto en este trabajo aún en desarrollo. 


\section{Conclusiones}

A lo largo de este trabajo de Tesis Doctoral estudiamos diversos procesos dinámicos sociales sobre redes bidimensionales y redes complejas de capas múltiples. Analizamos su fenomenología y las cantidades relevantes que los caracterizan.

En el capítulo 2 estudiamos un modelo basado en agentes en una red bidimensional que explora la competencia entre la persuasión y el compromiso en la formación de opiniones. Debido a las interacciones dentro de esta topología aparece una dinámica de coarsening cuyas propiedades dependen estrechamente de la relación $r=p / q$ entre las probabilidades de interacción de persuasión y compromiso.

Cuando el compromiso domina sobre la persuasión, inicialmente la dinámica es similar a la del VM, el coarsening no tiene tensión superficial y los dominios están formados por agentes moderados, ésto está asociado a un estado de opinión centralizado. El crecimiento de los dominios finalmente conduce a un estado donde todos los agentes tienen la misma orientación de opinión (positiva o negativa) hasta que se alcanza el consenso en un tiempo que escalea como $r^{-1} \ln N$ con el tamaño de la población $N$.

En el escenario opuesto, donde la persuasión domina sobre el compromiso, el coarsening es impulsado por la tensión superficial y los agentes moderados se ubican en la interfaz entre los dominios formados por los extremistas. Esto corresponde a un estado de opinión polarizado en el que la población se divide en dos grupos que adoptan opiniones extremas y opuestas (positiva y negativa). El tiempo para alcanzar el consenso puede ser muy largo cuando el sistema cae en una configuración metaestable tipo bandas, donde las dos interfases difunden hasta que se encuentran y aniquilan. El tiempo medio de consenso de este tipo de realizaciones escalea como $N^{1,71}$. Cuando el promedio se realiza sobre todas las realizaciones, que incluyen realizaciones de corta duración con un tiempo que escalea como $N$, el tiempo medio de consenso general escalea como $\tau \sim N^{\nu}$, con $\nu=1,64$. 
Se propuso una aproximación para describir el consenso de las configuraciones tipo bandas al mapear esta dinámica en un problema de dos barras que difunden libremente en $1 D$ y se aniquilan cuando chocan por primera vez. Este método tiene en cuenta el ancho de las bandas, que se vuelve relevante cuando éstas se encuentran y se rompen. Además, se realizó una aproximación más fina que explica las desviaciones del exponente teórico $\nu=1.5$, tomando en cuenta la rugosidad de las interfases, explicado en detalle en la subsección 2.4.2.

Respecto de los resultados conocidos del Modelo M en MF, las interacciones en la topología espacial de $2 D$ tienen un gran impacto. La bipolarización de opinión es mucho más estable en redes bidimensionales que en MF, debido a la existencia de estados metaestables de larga duración (estados banda). Otra observación es que la bipolarización se encuentra para $p>q$ en MF, mientras que en redes cuadradas bidimensionales se encuentra para valores de persuasión mucho más bajos, aproximadamente por $p>q / 3$. Por lo tanto, un pequeño nivel de homofilia es suficiente para inducir bipolarización en una población que interactúa en éste tipo de redes.

En redes cuadradas bidimensionales los tiempos medios de consenso son mucho más largos que los obtenidos en una configuración de MF y los estados bandas hacen que este tiempo sea aún mayor. Teniendo en cuenta las propiedades de escala del ancho de la interfase, se pudo derivar una expresión analítica para el tiempo medio de consenso con el tamaño del sistema de acuerdo con las simulaciones.

Valdría la pena estudiar la dinámica del modelo $\mathrm{M}$ en redes complejas de diferentes tipos ya que son descripciones más realistas de la topología de las interacciones sociales entre las personas. También sería interesante investigar el papel de la conectividad de red en la propagación y el dominio final de una opinión extrema 68. Finalmente, una extensión natural del modelo incluiría variaciones de las reglas en la persuasión y/o compromiso que podrían mejorar la bipolarización en redes o topologías generales.

En el capítulo 3, propusimos un modelo de red bicapa para explorar la interacción entre una dinámica de formación de opiniones y una de propagación de enfermedades en una población de individuos. Para simular la dinámica de opinión utilizamos el Modelo del Votante (VM) y para la dinámica de enfermedad utilizamos el Proceso de Contacto (CP) que se ejecutan en una red social y de contacto, respectivamente. Ambas redes comparten los mismos nodos y están unidos por una fracción $q$ de enlaces en común. Mostramos que, cuando las redes están acopladas, 
la dinámica de opinión puede cambiar drásticamente las propiedades estadísticas de la propagación de la enfermedad, que a su vez modifica las propiedades de la propagación de opiniones, en comparación con el caso de las redes aisladas $q=0$.

La dinámica del VM puede cambiar el orden de la transición, desde una transición continua para el caso desacoplado a una transición discontinua cuando el acoplamiento es $q>0$. La discontinuidad está asociada con la evolución temporal no monótona de la fracción de nodos infectados que depende de la probabilidad de infección efectiva, que varía con el tiempo según la evolución estocástica de la fracción de enlaces sociales +- . El sistema también muestra una transición discontinua de una fase endémica a una fase sana cuando el acoplamiento supera un valor $q_{c}$, para un valor fijo de $\beta$ cuyo origen es el mismo. Al realizar el diagrama de fases en el espacio $\beta-q$, observamos que el punto de transición $\beta_{c}$ aumenta con $q$.

También desarrollamos un enfoque de campo medio que permitió estimar con razonable precisión la línea de transición sana-endémica $\left(\beta_{c}, q_{c}\right)$ en función de los parámetros del modelo. El efecto general del VM en el CP es disminuir la probabilidad de infección efectiva a medida que aumenta el acoplamiento. Por lo tanto, cuando $q$ aumenta, se necesita un valor mayor de $\beta$ para llevar el sistema a la fase endémica, lo que lleva a un aumento del punto de transición $\beta_{c}$ con $q$.

Por su parte, la dinámica de CP tiene el efecto general de ralentizar la propagación de opiniones, retrasando el proceso de consenso de opinión en comparación con el observado en una red aislada. Dependiendo de los valores de los parámetros, el tiempo medio de consenso $\tau$ puede mostrar un comportamiento monótono o no monótono con $q$ que es el resultado de la competencia entre la superposición de enlaces sociales y de contacto que tiende a aumentar este tiempo con $q$ y la fracción de nodos infectados que tiende a disminuirlo con $q$.

Los resultados presentados en el capítulo 3 corresponden a un estado inicial particular que consiste en fracciones de pares de estados de opinión $+\mathrm{y}-\mathrm{y}$ de estados de enfermedad infectados $I$ y susceptibles $S$, distribuidos uniformemente a través de las redes. Como un trabajo futuro, se podría estudiar el comportamiento del sistema bajo diferentes condiciones iniciales, y con fracciones desiguales de opinión y estados de enfermedad. Por ejemplo, se puede simular una población con opiniones polarizadas iniciales basadas en la enfermedad, correlacionando la opinión de cada nodo con su estado de enfermedad (por ejemplo, infectando todos los nodos con opinión - y dejando todos los nodos con opinion + en estado saludable). 
Finalmente, sería interesante estudiar el comportamiento del modelo actual bajo diferentes reglas de actualización. Por ejemplo, hemos comprobado una regla simple en la que la condición de conexión (conectada o desconectada) entre dos nodos en una capa no se tiene en cuenta para la actualización en la otra capa con algunos resultados preliminares que sugieren que el comportamiento crítico de este nuevo modelo es bastante diferente al del modelo original.

En el capítulo 4 analizamos otro modelo de red bicapa para explorar la interacción entre la diseminación de una enfermedad y la propagación de información sobre el conocimiento de dicha enfermedad y sus métodos de prevención en una población de individuos mediante una aproximación de campo medio y el análisis de sus ecuaciones. En este modelo propuesto, el rumor y la enfermedad se propagan en diferentes escalas de tiempo. Verificamos que la información ayuda a reducir tanto el tamaño del brote como el umbral epidémico de la enfermedad, aunque un aumento en la velocidad de propagación de la información puede no ser beneficioso. Estas investigaciones pueden extenderse al considerar otros modelos de red que tomen en cuenta patrones de organización de comunidades, así como la organización de las mismas en otros tipos de Redes Multicapas.

Durante el desarrollo de la presente Tesis Doctoral fueron publicados los resultados expuestos en el Capítulo 2 que se completa con los apéndices A y B [104], en el capítulo 3 junto a los apéndices C, D, E y F [105], y se encuentra en preparación otro trabajo relativo a los estudios detallados en el Capítulo 4. 


\section{Apéndice A}

\section{Análisis de la distancia media de ruptura de la interfase $\left\langle\Delta x^{b}\right\rangle$}

Podemos obtener una idea del escaleo $\left\langle\Delta x^{b}\right\rangle \simeq L^{0.525}$ relacionando la distancia entre las interfases en el momento de ruptura con sus propiedades de rugosidad, como ilustramos en la Fig. 2.10(a). Más precisamente, las interfases se tocan a una altura $y\left(x_{1, y}=x_{2, y}=x_{y}\right)$ donde las respectivas desviaciones de la interfase de $x_{1}$ y $x_{2}$ alcanzan sus máximos valores en $d_{1, \max }=\left|x_{y}-x_{1}^{b}\right|$ y $d_{2, \max }=\left|x_{y}-x_{2}^{b}\right|$. Por lo tanto, la distancia entre las interfases se puede aproximar como $\Delta x^{b} \simeq d_{1, \max }+$ $d_{2, \text { max }}$. Dado que la altura $y$ del punto de contacto varía entre cada realización, calculamos el valor medio de la desviación máxima $\left\langle d_{m a x}\right\rangle$ en ambos lados de cada interfase para muchas realizaciones de la dinámica. Los resultados se muestran en la Fig. 2.11(a) (diamantes). Vemos que $2\left\langle d_{\max }\right\rangle$ coincide muy bien con $\left\langle\Delta x^{b}\right\rangle$ para $L \gtrsim 20$, y eso sigue una escala de ley de potencias $\left\langle d_{\max }\right\rangle \sim L^{0.525}$ (línea sólida). Especulamos que este comportamiento está relacionado con las propiedades de escala del ancho de las interfases, definido como la desviación estándar de las posiciones $x_{i, y}$ de la misma a lo largo del eje y 106, 107.

$$
W_{i}=\left[\frac{1}{L} \sum_{y=1}^{L} x_{i, y}^{2}-\left(\frac{1}{L} \sum_{y=1}^{L} x_{i, y}\right)^{2}\right]^{1 / 2}=\left[\frac{1}{L} \sum_{y=1}^{L}\left(x_{i, y}-x_{i}\right)^{2}\right]^{1 / 2} .
$$

La evolución temporal del ancho promedio de la interfase calculado sobre muchas realizaciones $\langle W\rangle$ [ver Fig. 2.11(b)] tiene una etapa inicial en la cual $\langle W\rangle$ crece como $t^{\beta}$, seguido por una segunda etapa donde $\langle W\rangle$ alcanza un valor de saturación (plateau) que se incrementa con $L$ como $\langle W\rangle_{\text {sat }}(L) \sim L^{\alpha}$, donde $\alpha \simeq 0.5$ es el exponente de rugosidad $\mathrm{y} \beta \simeq 0.25$ es el exponente de crecimiento. Estos exponentes son consistentes con los de la clase de universalidad Edwards-Wilkinson para el crecimiento de superficies [107. De hecho, mediante un reescaleo apropiado de 
los ejes $x$ e $y$, los datos pueden colapsarse en una sola función [Fig. 2.11(b)] que muestra que el crecimiento de la interfase obedece a la relación de escala FamilyVicsek $\langle W\rangle(L, t)=L^{\alpha} f\left(t / L^{z}\right)$ con $z=\alpha / \beta \simeq 2$, y $f(x) \sim x^{\beta}$ para $x \ll 1$ y $f(x)=$ constante $\simeq 0.22$ para $x \gg 1$. Observamos que el mismo comportamiento de escaleo de la dinámica de la interfase se obtuvo en [66] para una amplia familia de modelos de votantes con estados intermedios, como el modelo M. Además, verificamos que el ancho de la interfase haya alcanzado su valor de saturación en el tiempo medio de ruptura $\tau_{b}$ y que éste es mucho más largo que el "tiempo de cruce" que separa las etapas de crecimiento y saturación. Por lo tanto, se espera que la desviación máxima de la interfase sea proporcional al valor de saturación del ancho de la misma, lo que lleva al escaleo aproximado $\left\langle d_{\max }\right\rangle \sim L^{0.5}$. No sabemos cómo explicar la pequeña discrepancia con la escala $\left\langle d_{\max }\right\rangle \sim L^{0.525}$ obtenida de las simulaciones. 


\section{Apéndice B}

\section{Aproximación de $\tau_{b}^{\mathrm{III}}$ como una ley de potencia}

Para obtener el coeficiente $A\left(L_{0}\right)$ y el exponente $\alpha\left(L_{0}\right)$ de la aproximación de ley de potencia

$$
\tau_{b}^{\mathrm{III}}\left(L, L_{0}\right) \simeq A\left(L_{0}\right) L^{\alpha\left(L_{0}\right)}
$$

para $\tau_{b}^{\mathrm{III}}$ de la Ec. 2.10 resulta útil trabajar en una escala doble logarítmica, donde la Ec. (B.1) se convierte en la línea recta

$$
y\left(x, x_{0}\right) \simeq \log \left[A\left(x_{0}\right)\right]+\alpha\left(x_{0}\right) x
$$

en la variable $x \equiv \log (L)$, con $x_{0} \equiv \log \left(L_{0}\right)$ y $y\left(x, x_{0}\right) \equiv \log \left[\tau_{b}^{\mathrm{III}}\left(L, L_{0}\right)\right]$. Luego, reescribiendo la Ec. (2.10) en términos de las variables $x$ e $y(x)$

$$
y(x)=2 \log \left(1-2 e^{-0.475 x}\right)+3 x-\log (16 d),
$$

y expandiendo en serie de Taylor $y(x)$ a primer orden en $x-x_{0}$ obtenemos

$$
\begin{aligned}
y(x) & \simeq 2 \log \left(1-2 e^{-0.475 x_{0}}\right)+\frac{1.9\left(x-x_{0}\right)}{\left(e^{0.475 x_{0}}-2\right)}+3 x-\log (16 d) \\
& =\log \left[\frac{\left(1-2 e^{-0.475 x_{0}}\right)^{2}}{16 d}\right]-\frac{1.9 x_{0}}{\left(e^{0.475 x_{0}}-2\right)}+\left[3+\frac{1.9}{\left(e^{0.475 x_{0}}-2\right)}\right] x .
\end{aligned}
$$

Igualando los coeficientes de la Ec. (B.3) con los de la Ec. B.2 y devolviendo la transformación de la variable $L_{0}=e^{x_{0}}$, llegamos a las expresiones para $A\left(L_{0}\right)$ y $\alpha\left(L_{0}\right)$ citadas en las Ecs. 2.13) y 2.14), respectivamente, en el capítulo 2 de esta tesis. 



\section{Apéndice $\mathrm{C}$}

\section{Deducción de la ecuación de evolución para $\rho^{+}$}

Denotamos por $\left[\begin{array}{l}\mathcal{O} \\ \mathcal{D}\end{array}\right]$ el estado de un nodo dado, donde $\mathcal{O}=+,-$ y $\mathcal{D}=1,0$ representan sus estados de opinión y enfermedad, respectivamente. Por lo tanto, hay cuatro posibles estados de nodo: $\left[\begin{array}{l}+ \\ 0\end{array}\right],\left[\begin{array}{l}+ \\ 1\end{array}\right],\left[\begin{array}{c}- \\ 0\end{array}\right]$ y $\left[\begin{array}{c}- \\ 1\end{array}\right]$. En un solo paso de la dinámica, las transiciones del estado $\left[\begin{array}{c}- \\ \mathcal{D}\end{array}\right]$ al estado $\left[\begin{array}{l}+ \\ \mathcal{D}\end{array}\right]$ cuando un nodo cambia de opinión - a + conduce a una ganancia de $1 / N$ en $\rho^{+}$, mientras que las transiciones $\left[\begin{array}{l}+ \\ \mathcal{D}\end{array}\right] \rightarrow\left[\begin{array}{c}- \\ \mathcal{D}\end{array}\right]$ cuando hay un cambio de opinión $+\rightarrow-$ conduce a una pérdida de $1 / N$ en $\rho^{+}$. Teniendo en cuenta estas cuatro posibles transiciones, el cambio promedio de $\rho^{+}$en un solo paso de tiempo del intervalo temporal $\Delta t=1 / N$ es descrita por la ecuación de evolución

$$
\begin{aligned}
\frac{d \rho^{+}}{d t} & =\left.\frac{d \rho^{+}}{d t}\right|_{-\rightarrow+}+\left.\frac{d \rho^{+}}{d t}\right|_{+\rightarrow-} \\
& =\frac{1}{1 / N}\left[\left.\Delta \rho^{+}\right|_{-_{0} \rightarrow_{0}^{+}}+\left.\Delta \rho^{+}\right|_{-_{1} \rightarrow_{1}^{+}}+\left.\Delta \rho^{+}\right|_{+_{0} \rightarrow 0_{0}^{-}}+\left.\Delta \rho^{+}\right|_{1} \rightarrow_{1}^{-}\right]
\end{aligned}
$$

donde, por ejemplo, el término $\left.\Delta \rho^{+}\right|_{0_{0} \rightarrow_{0}^{+}}$representa el cambio promedio de $\rho^{+}$en un paso de tiempo debido a las transiciones $\left[\begin{array}{c}- \\ 0\end{array}\right] \rightarrow\left[\begin{array}{c}+ \\ 0\end{array}\right]$. A su vez, $\left.\Delta \rho^{+}\right|_{0_{0} \rightarrow_{0}^{+}}$tiene cuatro contribuciones correspondientes a las diferentes interacciones sociales que conducen a la transición $\left[\begin{array}{l}- \\ 0\end{array}\right] \rightarrow\left[\begin{array}{l}+ \\ 0\end{array}\right]$. Por lo tanto, podemos escribir

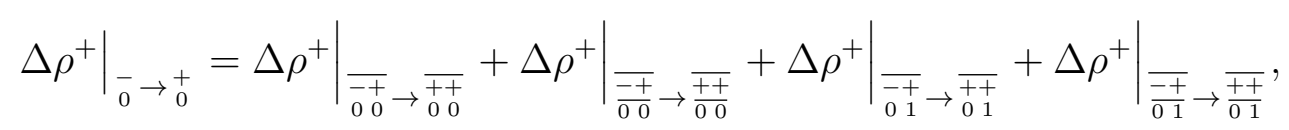

y similarmente para la transición $\left[\begin{array}{l}- \\ 1\end{array}\right] \rightarrow\left[\begin{array}{l}+ \\ 1\end{array}\right]$ correspondiente al segundo término en la Ec. (C.1)

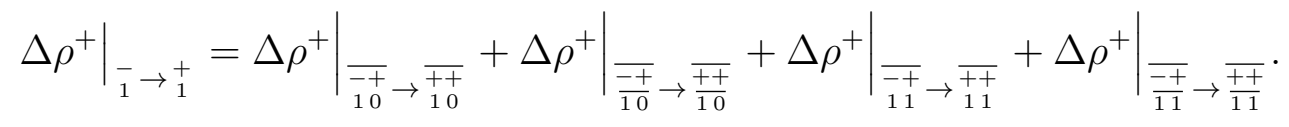


El tercer y cuarto término en la Ec. C.1 se obtienen intercambiando los símbolos $+\mathrm{y}$ - en las Ecs. C.2 y C.3), respectivamente, debido a la simetría entre los estados de opinión $+\mathrm{y}-$. Observamos que los estados de enfermedad siguen siendo los mismos después de las interacciones, ya que solo un cambio en la capa social puede conducir a un cambio en $\rho^{+}$. El primer término en la Ec. C.2 representa el cambio promedio en $\rho^{+}$debido a interacciones en las que un nodo $i$ en estado $\left[\begin{array}{c}- \\ 0\end{array}\right]$ copia la opinión de uno de sus vecinos sociales $j$ en estado $\left[\begin{array}{l}+ \\ 0\end{array}\right]$, cambiando el estado de $i$ a $\left[\begin{array}{c}+ \\ 0\end{array}\right]$. Esta interacción está representada esquemáticamente por el símbolo $\left[\begin{array}{c}\overline{-+} \\ 0\end{array}\right]$, donde la línea horizontal sobre los símbolos de opinión describe un link social entre $i$ y $j$. De la misma forma, el símbolo $\left[\frac{\overline{-+}}{00}\right]$ representa una interacción entre un nodo $\left[\begin{array}{c}- \\ 0\end{array}\right]$ y un nodo vecino $\left[\begin{array}{l}+ \\ 0\end{array}\right]$ conectados por un link social y un link de contacto, esto se indica con una línea horizontal sobre los respectivos símbolos. El segundo, tercero y cuarto término en la Ec. C.2 describen, respectivamente, las transiciones debidas a una interacción del nodo $i$ con un vecino social/de contacto $\left[\begin{array}{l}+ \\ 0\end{array}\right]$, un vecino social $\left[\begin{array}{l}+ \\ 1\end{array}\right]$ y un vecino social/de contacto $\left[\begin{array}{l}+ \\ 1\end{array}\right]$.

Ahora ilustraremos cómo construir una expresión aproximada para cada término de la Ec. C.2 para $\left.\Delta \rho^{+}\right|_{0_{0} \rightarrow_{0}^{+}}$. En aras de la simplicidad, suponemos que todos los nodos tienen el mismo número de vecinos $k=\mu$ elegidos al azar, lo que equivale a suponer que las redes son gráfos aleatorios de grado regular. Sin embargo, esperamos que esta aproximación funcione bien en redes con distribuciones de grados homogéneas como las redes ER. El primer término en la Ec. C.2 puede escribirse como

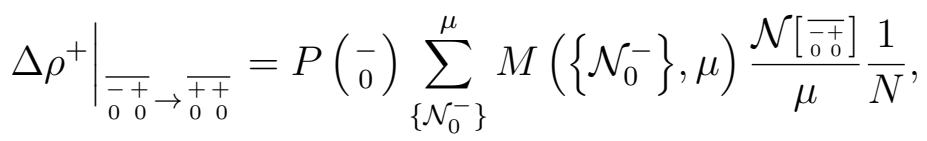

que puede entenderse como el producto de las diferentes probabilidades asociadas a cada uno de los sucesos consecutivos que conducen a la transición en un paso de tiempo $\left[\begin{array}{l}\overline{-+} \\ 0\end{array}\right] \rightarrow\left[\begin{array}{l}\overline{++} \\ 0\end{array}\right]$, como describiremos a continuación. Un nodo $i$ con estado $\left[\begin{array}{c}- \\ 0\end{array}\right]$ es escogido al azar con probabilidad $P\left(\begin{array}{c}- \\ 0\end{array}\right)$. Si el nodo $i$ tiene $\mathcal{N}\left[\begin{array}{c}-\overline{-} \\ 0\end{array}\right]$ vecinos sociales con estado $\left[\begin{array}{c}+ \\ 0\end{array}\right]$, entonces uno de esos vecinos sociales $j$ se escoge al azar con probabilidad $\mathcal{N}\left[\begin{array}{c}\overline{-}+ \\ 0\end{array}\right] / \mu$, después de lo cual $i$ copia la opinión de $j$ con probabilidad 1.0 porque no existe link de contacto entre $i$ y $j$. Finalmente, $\rho^{+}$se incrementa en $1 / N$ cuando $i$ cambia su opinión.

Para considerar todos los escenarios posibles de tener $\mathcal{N}\left[\begin{array}{c}-+ \\ \left.-\begin{array}{c}+ \\ 0\end{array}\right]\end{array}\right]=0,1, . ., \mu$ vecinos sociales, sumamos todas las configuraciones de vecindarios posibles representadas

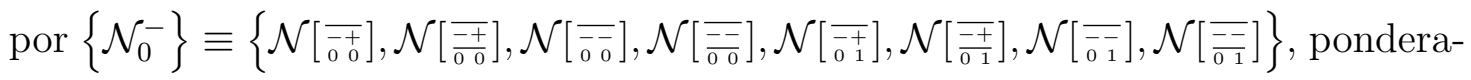




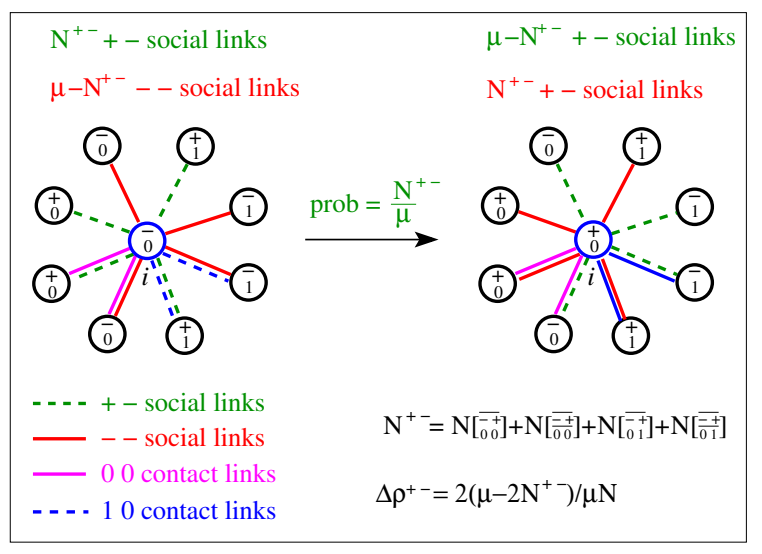

Figura C.1: (Color online) Ilustración esquemática de una actualización de opinión en la que un nodo $i$ en estado $\left[\begin{array}{c}- \\ 0\end{array}\right]$ cambia al estado $\left[\begin{array}{l}+ \\ 0\end{array}\right]$ copiando la opinión + de un vecino escogido aleatoriamente (links verdes punteados). El cambio en la densidad de links +- es denotado por $\Delta \rho^{+-}$.

do por la probabilidad de cada configuración $M\left(\left\{\mathcal{N}_{0}^{-}\right\}, \mu\right)$. Aquí denotamos por $\mathcal{N}\left[\begin{array}{c}\overline{\bar{O}} \\ 0\end{array}\right]$ el número de vecinos sociales $\left[\begin{array}{l}\mathcal{O} \\ \mathcal{D}\end{array}\right]$ y por $\mathcal{N}\left[\frac{\overline{-O}}{\overline{0} \mathcal{D}}\right]$ el número de vecinos sociales/de contacto $\left[\begin{array}{l}\mathcal{O} \\ \mathcal{D}\end{array}\right]$ [ver Fig. C.1]]. El número de cada tipo de vecino está entre 0 y $\mu$, y así la suma en la Ec. (C.4) incluye ocho sumas

$$
\sum_{\left\{\mathcal{N}_{0}^{-}\right\}}^{\mu} \equiv \sum_{\substack{\mathcal{O}=+,-\mathcal{D}=0,1}}\left(\sum_{\mathcal{N}\left[\frac{\bar{O}}{0 \mathcal{O}}\right]=0}^{\mu}+\sum_{\mathcal{N}\left[\frac{\overline{-O}}{\overline{0} \mathcal{D}}\right]=0}^{\mu}\right)
$$

sobre todas las combinaciones sujetas a la restricción

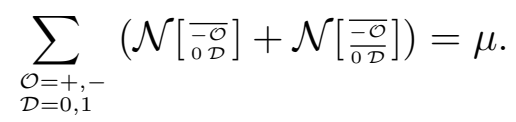

Para llevar a cabo la suma en la Ec. (C.4 solo tendremos en cuenta las correlaciones entre los primeros vecinos, y descuidamos las correlaciones de segundo y mayor orden (aproximación de pares). Por lo tanto, definimos la probabilidad $P\left[\begin{array}{c}\overline{-\mathcal{O}} \\ 0 \mathcal{D}\end{array}\right] \equiv P\left(\begin{array}{r|r}-\mathcal{O} & - \\ \mathcal{D}\end{array}\right)$ de que un vecino dado del nodo $i$ es un vecino social con estado $\left[\begin{array}{l}\mathcal{O} \\ \mathcal{D}\end{array}\right]$, y consideramos que $P\left[\begin{array}{c}\overline{-\mathcal{O}} \\ 0 \mathcal{D}\end{array}\right]$ está condicionada solamente al estado de $i\left[\begin{array}{c}- \\ 0\end{array}\right]$, y no a los otros vecinos de $i$. Del mismo modo, denotamos por $P\left[\overline{\frac{-\mathcal{O}}{0 \mathcal{D}}}\right]=P\left(\begin{array}{cc|c}-\mathcal{O} & - \\ -\mathcal{D} & 0\end{array}\right)$ a la probabilidad condicional de que un nodo conectado a $i$ es un vecino social/de contacto con estado $\left[\begin{array}{l}\mathcal{O} \\ \mathcal{D}\end{array}\right]$, dado que $i$ tiene estado $\left[\begin{array}{c}- \\ 0\end{array}\right]$. Por lo tanto, $M$ se convierte 
en la distribución de probabilidad multinomial definida como

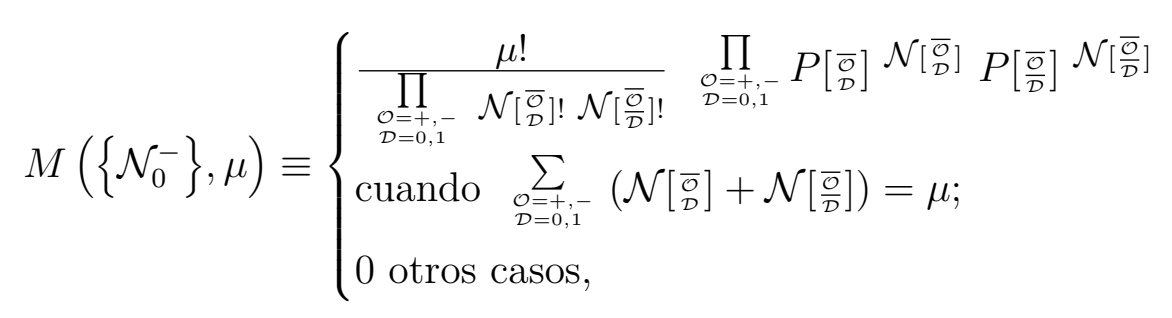

donde hemos usado los símbolos $\left[\begin{array}{l}\overline{\mathcal{O}} \\ \mathcal{D}\end{array}\right]$ y $\left[\begin{array}{l}\overline{\mathcal{O}} \\ \mathcal{D}\end{array}\right]$ como notaciones cortas para $\left[\begin{array}{c}\overline{-\mathcal{O}} \\ 0\end{array}\right]$ y $\left[\frac{\overline{-\mathcal{O}}}{\overline{0 D}}\right]$, respectivamente. Luego, realizando la suma de la Ec. C.4 llegamos a

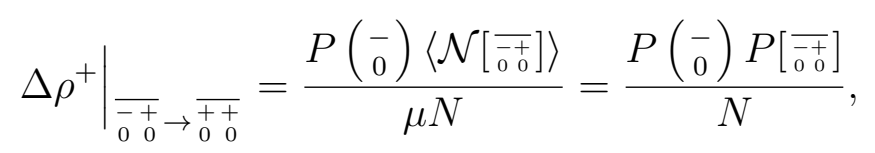

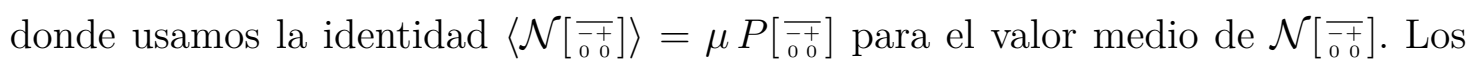
otros tres términos de la Ec. (C.2) pueden ser obtenidos siguiendo un enfoque similar al anterior para $\left.\Delta \rho^{+}\right|_{\substack{0_{0}^{+} \\ \overline{0} \rightarrow+0_{0}^{++}}}$, llegando a la expresión

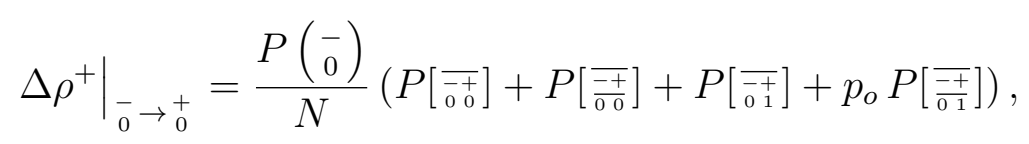

donde el prefactor $p_{o}$ en el último término explica la probabilidad de copiar la opinión de un vecino de contacto infectado. Teniendo en cuenta que nuestro objetivo es obtener un sistema cerrado de ecuaciones de evolución para $\rho^{+}, \rho_{1}, \rho^{+-}$y $\rho_{10}$, ahora encontramos expresiones aproximadas para las diferentes probabilidades de la Ec. (C.6) en términos de las fracciones de nodos y links en cada capa. Comenzamos por suponer que las correlaciones entre los estados de opinión y los estados de enfermedad de un nodo dado son insignificantes, y así podemos escribir

$$
P\left(\begin{array}{c}
- \\
0
\end{array}\right) \simeq \rho^{-} \rho_{0}
$$

Entonces, para estimar las probabilidades condicionales $P\left[\begin{array}{c}\overline{-\overline{0}} \\ 0 .\end{array}\right]$ y $P\left[\overline{\frac{-+}{0 \mathcal{D}}}\right]$ resulta conveniente dividir cada uno de ellos en dos probabilidades condicionales

$$
\begin{aligned}
& P\left[\begin{array}{c}
\overline{-+} \\
\overline{0} \mathcal{D}
\end{array}\right]=P\left(-{ }_{\mathcal{D}}^{+} \mid \begin{array}{l}
- \\
0
\end{array}\right)=P\left(-\mid \begin{array}{c}
- \\
0
\end{array}\right) P\left(\begin{array}{c}
+ \\
\mathcal{D}
\end{array} \mid-\frac{-}{0}\right),
\end{aligned}
$$

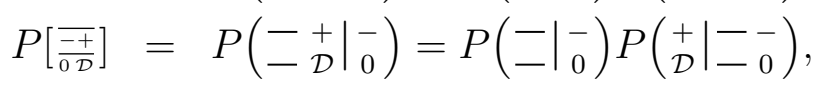

usando la relación $P(a, b \mid c)=P(a \mid c) P(b \mid a, c)$ interpretando todo el evento de conectar un tipo dado de enlace a un nodo $\left[{ }_{\mathcal{D}}^{+}\right]$como dos eventos separados. Suponiendo que el tipo de enlace conectado al nodo $i$ no está correlacionado con el estado de $i$, tenemos

$$
\begin{aligned}
& P\left(-\mid \begin{array}{c}
- \\
0
\end{array}\right) \simeq P(-)=1-q \quad y \\
& P\left(\text { Z }_{0}^{-}\right) \simeq P\left(\begin{array}{l}
- \\
-
\end{array}\right)=q,
\end{aligned}
$$


y como los estados de opinión y enfermedad no están correlacionados, tenemos

$$
\begin{aligned}
& P\left(\begin{array}{l}
+ \\
\mathcal{D}
\end{array} \mid-\frac{-}{0}\right) \simeq P(+\mid--) P(\mathcal{D} \mid 0) \quad \mathrm{y} \\
& P\left(\begin{array}{l}
+ \\
\mathcal{D}
\end{array} \mid-\overline{-}\right) \simeq P(+\mid--) P(\mathcal{D} \mid-0) .
\end{aligned}
$$

Dentro de una aproximación de pares homogénea [6], la probabilidad $P(+\mid--)$ que un vecino social $j$ de un nodo $i$ con opinión $\mathcal{O}_{i}=-$ tenga opinión $\mathcal{O}_{j}=+$ se puede estimar como la relación entre el número total de links $\mu N \rho^{+-} / 2$ que van de nodos con opinión $-\mathrm{a}+\mathrm{y}$ el número total de links $\mu N \rho^{-}$conectados a los nodos con opinión - , esto es $P(+\mid--) \simeq \rho^{+-} / 2 \rho^{-}$. Del mismo modo, estimamos que la probabilidad de que un vecino de contacto $j$ de un nodo susceptible tenga un estado de enfermedad $\mathcal{D}_{j}=0$ como $P(0 \mid-0) \simeq \rho_{00} / \rho_{0}$, y un estado de enfermedad $\mathcal{D}_{j}=1$ como $P(1 \mid-0) \simeq \rho_{10} / 2 \rho_{0}$. Y si $j$ no es vecino de $i$ en la red de contacto $P(\mathcal{D} \mid \quad 0) \simeq \rho_{\mathcal{D}}$. Reuniendo todos estos factores obtenemos

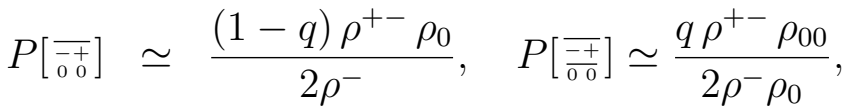

$$
\begin{aligned}
& P\left[\begin{array}{c}
\overline{\overline{0}}+\overline{1} \\
0
\end{array}\right] \simeq \frac{(1-q) \rho^{+-} \rho_{1}}{2 \rho^{-}}, \quad P[\overline{\overline{-+}}] \simeq \frac{q \rho^{+-} \rho_{10}}{4 \rho^{-} \rho_{0}} .
\end{aligned}
$$

Finalmente, reemplazando en la Ec. (C.6) las expresiones aproximadas para las probabilidades condicionales de las Ecs. (C.8) y para $P\left(\begin{array}{c}- \\ 0\end{array}\right)$ de la Ec. (C.7) llegamos a

$$
\left.\Delta \rho^{+}\right|_{-\rightarrow_{0}^{+}}=\frac{\rho^{+-}}{2 N}\left[\rho_{0}-q\left(1-p_{o}\right) \frac{\rho_{10}}{2}\right]
$$

donde usamos las relaciones de conservación de las Ecs. (3.2a) y (3.2d).

Ahora calculamos el segundo término de ganancia en la Ec. C.1,$\left.\Delta \rho^{+}\right|_{-} \rightarrow_{1}^{+}$, que representa el cambio promedio en $\rho^{+}$debido a las transiciones $\left[\begin{array}{l}- \\ 1\end{array}\right] \rightarrow\left[\begin{array}{l}+ \\ 1\end{array}\right]$, siguiendo los mismos pasos utilizado anteriormente para el término $\left.\Delta \rho^{+}\right|_{{ }_{0} \rightarrow_{0}^{+}}$. De la Ec. C.3) obtenemos

$$
\begin{aligned}
& \left.\Delta \rho^{+}\right|_{{ }_{1} \rightarrow_{1}^{+}}=P\left(\frac{-}{1}\right) \sum_{\left\{\mathcal{N}_{1}^{-}\right\}}^{\mu} M\left(\left\{\mathcal{N}_{1}^{-}\right\}, \mu\right) \frac{1}{\mu}\left\{\mathcal{N}\left[\begin{array}{c}
\overline{-+} \\
10
\end{array}\right]+p_{o} \mathcal{N}\left[\frac{\overline{-+}}{10}\right]+\mathcal{N}\left[\overline{\frac{-+}{11}}\right]+p_{o} \mathcal{N}\left[\frac{\overline{\frac{-}{11}}}{11}\right]\right\} \frac{1}{N}
\end{aligned}
$$

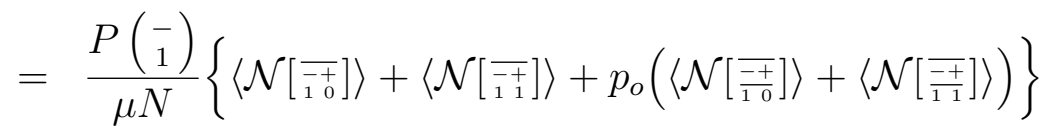

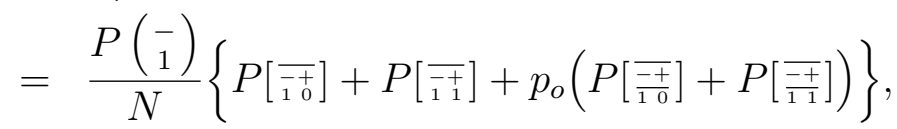


y usando las aproximaciones

$$
\begin{aligned}
& P\left[\begin{array}{c}
\overline{-+} \\
10
\end{array}\right] \simeq \frac{(1-q) \rho^{+-} \rho_{0}}{2 \rho^{-}}, \quad P\left[\frac{\overline{-+}}{10}\right] \simeq \frac{q \rho^{+-} \rho_{10}}{4 \rho^{-} \rho_{1}} \\
& P\left[\begin{array}{c}
\overline{-+} \\
11
\end{array}\right] \simeq \frac{(1-q) \rho^{+-} \rho_{1}}{2 \rho^{-}}, \quad P\left[\frac{-+}{11}\right] \simeq \frac{q \rho^{+-} \rho_{11}}{2 \rho^{-} \rho_{1}}
\end{aligned}
$$

para las probabilidades condicionales llegamos a

$$
\left.\Delta \rho^{+}\right|_{-\rightarrow_{1}^{+}}=\frac{\rho^{+-} \rho_{1}}{2 N}\left[1-q\left(1-p_{o}\right)\right]
$$

donde hemos usado las relaciones de conservación de las Ecs. (3.2a) y (3.2c).

Agregando las Ecs. C.9 y C.11 obtenemos la siguiente expresión para el término de ganancia media de un nodo con opinión + en un solo paso de tiempo, correspondiente a la suma de los primeros y segundos términos de la Ec. (C.1)

$$
\left.\frac{d \rho^{+}}{d t}\right|_{-\rightarrow+}=\frac{1}{1 / N}\left[\left.\Delta \rho^{+}\right|_{-_{-} \rightarrow_{0}^{+}}+\left.\Delta \rho^{+}\right|_{-_{1} \rightarrow_{1}^{+}}\right] \simeq \frac{1}{2} \omega \rho^{+-},
$$

con

$$
\omega \equiv 1-q\left(1-p_{o}\right)\left(\rho_{1}+\frac{\rho_{10}}{2}\right)
$$

El prefactor $\omega$ juega un importante rol en la dinámica del consenso de opiniones, al establecer la escala de tiempo asociada a las actualizaciones de opinión, y puede interpretarse como una probabilidad efectiva de que un nodo adopte la opinión de un vecino con opinión opuesta, elegido al azar. Es decir, la Ec. C.12 de ganancia de un nodo con opinión + simplemente describe el proceso de selección de un nodo $i$ con opinión $-\mathrm{y}$ un vecino $j$ con opinión + , lo que ocurre con probabilidad $\rho^{+-} / 2$, y luego el nodo $i$ cambiará su opinión con probabilidad $\omega$ que depende del tipo de conexión y el estado de enfermedad de ambos $(i$ y $j$ ). Esta "probabilidad efectiva de copiado" $\omega$ resulta ser una probabilidad de copia promedio en toda la red social, como se muestra en la sección 3.3 .

Para encontrar la ecuación para la pérdida promedio de un nodo con opinión + en un paso de tiempo, que corresponde a la suma del tercer y cuarto término de la Ec. (C.1), podemos explorar la simetría entre los estados de opinión + y - y simplemente intercambiar los signos $+\mathrm{y}-$ en la Ec. (C.12)

$$
\left.\frac{d \rho^{+}}{d t}\right|_{+\rightarrow-}=\frac{1}{1 / N}\left[\left.\Delta \rho^{+}\right|_{0} ^{+\rightarrow_{0}^{-}}+\left.\Delta \rho^{+}\right|_{+_{1} \rightarrow_{1}^{-}}\right] \simeq-\frac{1}{2} \omega \rho^{+-}
$$

donde usamos $\rho^{-+}=\rho^{+-}$. Finalmente, sumando las Ecs. C.12) y C.14 obtenemos

$$
\frac{d \rho^{+}}{d t}=0
$$


este resultado fue mencionado en la Ec. (3.3a) del capítulo 3 de esta tesis. Por lo tanto, las fracciones de nodos $+\mathrm{y}-$ se conservan en todo momento: $\rho^{+}(t)=\rho^{+}(0)$ y $\rho^{-}(t)=\rho^{-}(0)=1-\rho^{+}(0)$. Aunque el cálculo anterior conduce a un resultado muy simple, sirve como introducción a la metodología utilizada para derivar ecuaciones de evolución para las otras fracciones $\rho^{+-}, \rho_{1}$ y $\rho_{10}$, como mostramos en los siguientes apéndices. 



\section{Apéndice D}

\section{Deducción de la ecuación de evolución para $\rho^{+-}$}

En analogía al cálculo de $\rho^{+}$en el apéndice C, el cambio promedio de la fracción de links sociales $\rho^{+-}$en un paso de tiempo viene dado por la ecuación de evolución

$$
\frac{d \rho^{+-}}{d t}=\left.\frac{d \rho^{+-}}{d t}\right|_{-\rightarrow+}+\left.\frac{d \rho^{+-}}{d t}\right|_{+\rightarrow-}
$$

con

$$
\begin{aligned}
\left.\frac{d \rho^{+-}}{d t}\right|_{-\rightarrow+} & =\frac{1}{1 / N}\left[\left.\Delta \rho^{+-}\right|_{-_{0} \rightarrow_{0}^{+}}+\left.\Delta \rho^{+-}\right|_{-_{1} \rightarrow_{1}^{+}}\right] \\
\left.\frac{d \rho^{+-}}{d t}\right|_{+\rightarrow-} & =\frac{1}{1 / N}\left[\left.\Delta \rho^{+-}\right|_{0} ^{+\rightarrow_{0}^{-}}+\left.\Delta \rho^{+-}\right|_{1 \rightarrow T_{1}^{-}}\right]=\left\{\left.\frac{d \rho^{+-}}{d t}\right|_{-\rightarrow+}\right\}^{-\Longleftrightarrow+}
\end{aligned}
$$

donde el símbolo $-\Longleftrightarrow+$ indica el intercambio de signos $+\mathrm{y}-$ en la expresión entre llaves. La Ec. (D.3) significa que la simetría entre las opiniones + y - permite encontrar el segundo término en la Ec. (D.1) intercambiando los signos en el primer término. Para calcular el primer término en la Ec. (D.2) sumamos los cuatro tipos de interacciones $\left[\begin{array}{c}- \\ 0\end{array}\right]$ de un nodo $i$ con un vecino $j$ con estado $\left[\begin{array}{l}+ \\ \mathcal{D}\end{array}\right]$ que conduce a la transición $\left[\begin{array}{c}- \\ 0\end{array}\right] \rightarrow\left[\begin{array}{l}+ \\ 0\end{array}\right]$

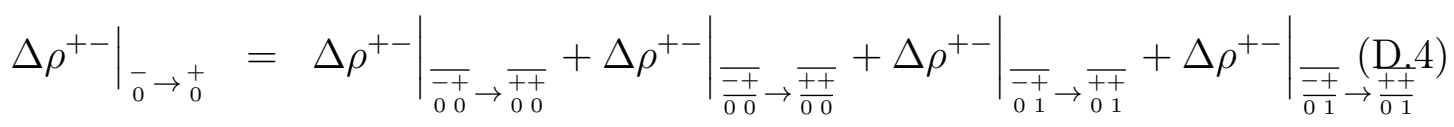

Como se explicó en el apéndice C, las probabilidades de interacción $\left[\begin{array}{l}\overline{-+} \\ 0 \mathcal{D}\end{array}\right]$ y $\left[\begin{array}{l}\overline{\frac{-+}{0 \mathcal{D}}}\end{array}\right]$ son dadas por las fracciones respectivas $\mathcal{N}\left[\begin{array}{c}\overline{-+} \\ 0 \mathcal{D}\end{array}\right] / \mu$ y $\mathcal{N}\left[\frac{\overline{-+}}{\frac{-D}{0 D}}\right] / \mu$ de cada tipo de vecino. El cambio en el número de links sociales +- después de que el nodo $i$ cambia su estado de opinión es dado por la expresión $\mu-2\left(\mathcal{N}\left[\begin{array}{c}\overline{-+} \\ 0\end{array}\right]+\mathcal{N}\left[\overline{\frac{-+}{00}}\right]+\mathcal{N}\left[\begin{array}{c}\overline{-+} \\ 01\end{array}\right]+\mathcal{N}\left[\frac{\overline{-}}{\overline{01}}\right]\right)$, que toma en cuenta la configuración específica de enlaces y vecinos conectados a $i$, 
como se muestra en la Fig. C.1. Obtenemos

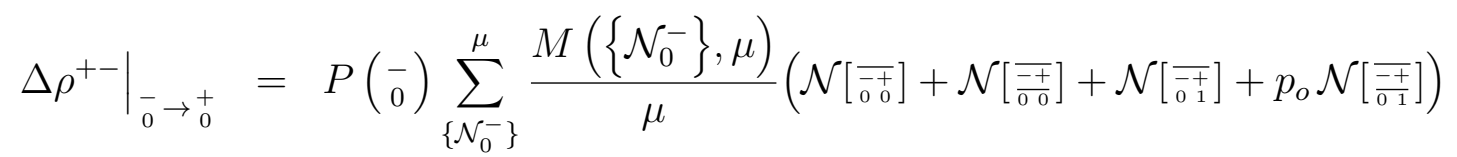

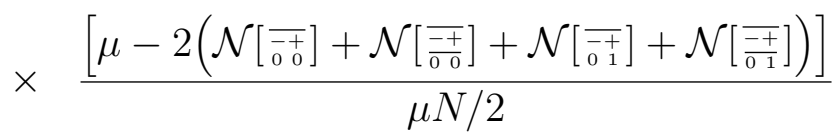

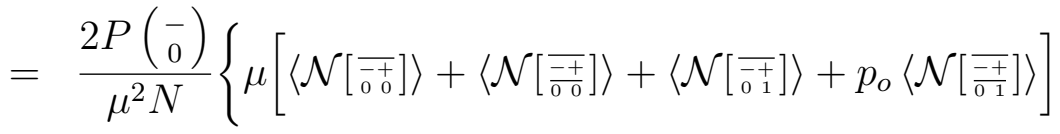

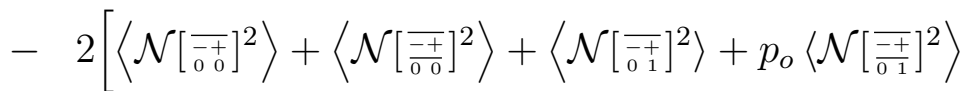

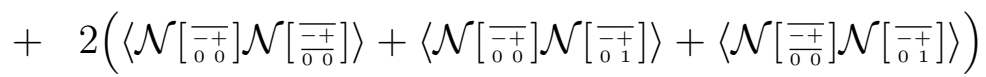

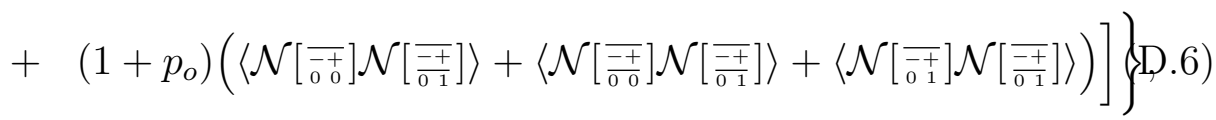

donde el primer y segundo momento de $M\left(\left\{\mathcal{N}_{0}^{-}\right\}, \mu\right)$ son

$$
\begin{aligned}
& \left.\left\langle\mathcal{N}\left[\overline{\overline{\mathcal{D}_{i} \mathcal{D}_{j}}}\right]\right\rangle=\mu P\left[\overline{\overline{\mathcal{D}_{i}}}\right]+\underset{\mathcal{D}_{j}}{+}\right], \\
& \left\langle\mathcal{N}\left[\frac{\overline{-+}}{\overline{\mathcal{D}_{i} \mathcal{D}_{j}}}\right]\right\rangle=\mu P\left[\frac{\overline{\overline{\mathcal{D}_{i}}}}{\overline{\mathcal{D}_{j}}}\right], \\
& \left.\left\langle\mathcal{N}\left[\overline{\overline{\mathcal{D}_{i} \mathcal{D}_{j}}}\right]^{+}\right\rangle=\mu P\left[\overline{\overline{\mathcal{D}_{i} \mathcal{D}_{j}}}\right]+\mu(\mu-1) P\left[\overline{\overline{\bar{D}_{i} \mathcal{D}_{j}}}\right]^{+}\right]^{2}, \\
& \left\langle\mathcal{N}\left[\frac{\overline{\overline{\mathcal{D}_{i} \mathcal{D}_{j}}}}{\overline{D^{\prime}}}\right]^{2}\right\rangle=\mu P\left[\overline{\overline{\mathcal{D}_{i} \mathcal{D}_{j}}}\right]+\mu(\mu-1) P\left[\overline{\frac{-+}{\overline{\mathcal{D}_{i}} \mathcal{D}_{j}}}\right]^{2},
\end{aligned}
$$

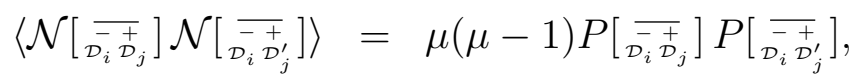

$$
\begin{aligned}
& \left\langle\mathcal{N}\left[\frac{\overline{\frac{-+}{\overline{\mathcal{D}_{i}}}}}{\mathcal{D}_{j}}\right] \mathcal{N}\left[\frac{\overline{-+}}{\overline{\mathcal{D}_{i} \mathcal{D}_{j}^{\prime}}}\right]\right\rangle=\mu(\mu-1) P\left[\frac{\overline{-+}}{\overline{\bar{D}_{i} \mathcal{D}_{j}}}\right] P\left[\frac{\overline{-+}}{\overline{\mathcal{D}_{i} \mathcal{D}_{j}^{\prime}}}\right],
\end{aligned}
$$

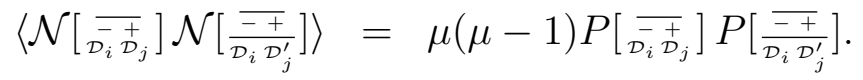

Aquí $\mathcal{D}_{i}=1.0$ y $\mathcal{D}_{j}=1.0$ son los estados de enfermedad de los nodos $i$ y $j$, respectivamente. Replazando las expresiones para los momentos de las Ecs. (D.7) en la Ec. (D.6) y reagrupando los términos, obtenemos

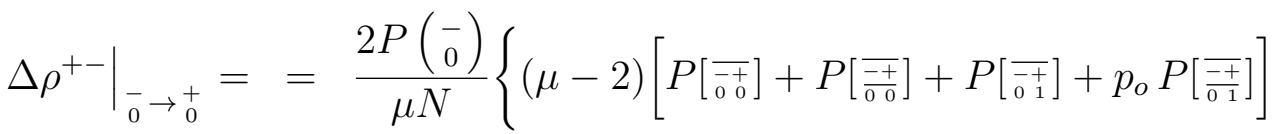

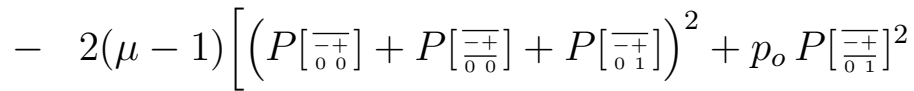

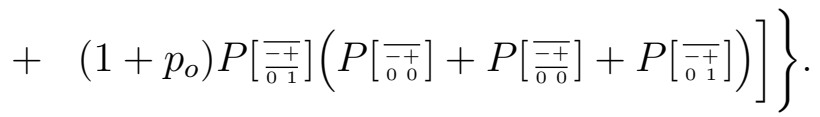

Sustituyendo las expresiones para las probabilidades $P\left[\begin{array}{c}\overline{-\bar{D}} \\ 0\end{array}\right]$ y $P\left[\overline{\frac{-+}{0 D}}\right]$ de la Ec. (C.8) en la Ec. (D.8), y después de realizar un poco de álgebra, finalmente obtenemos

$$
\begin{array}{r}
\left.\Delta \rho^{+-}\right|_{0} \rightarrow_{0}^{+}=\frac{\rho^{+-}}{\mu N}\left\{(\mu-2)\left[(1-q) \rho_{0}+q\left(\rho_{00}+p_{o} \frac{\rho_{10}}{2}\right)\right]-\right. \\
\left.(\mu-1) \frac{\rho^{+-}}{\rho^{-}}\left[\rho_{0}-\left(1-p_{o}\right) q \frac{\rho_{10}}{2}\right]\right\} .
\end{array}
$$


Ahora seguimos un enfoque similar al anterior para $\left.\Delta \rho^{+-}\right|_{{ }_{0}} \rightarrow_{0}^{+}$y calculamos el segundo término de la Ec. (D.2) como

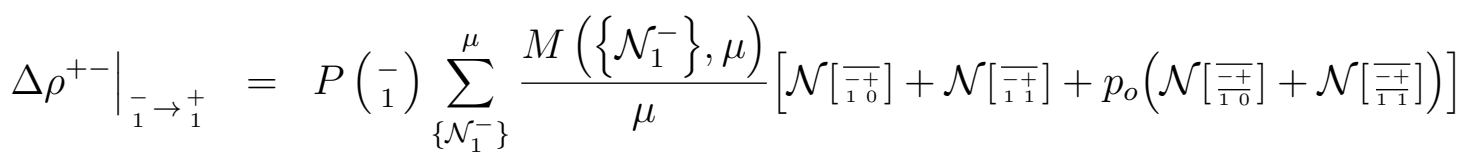

$$
\begin{aligned}
& \times \frac{\left[\mu-2\left(\mathcal{N}\left[\begin{array}{c}
\overline{-+} \\
10
\end{array}\right]+\mathcal{N}\left[\begin{array}{c}
\overline{\frac{-t}{10}} \\
\frac{10}{0}
\end{array}\right]+\mathcal{N}\left[\begin{array}{c}
\overline{-+} \\
11
\end{array}\right]+\mathcal{N}\left[\frac{\overline{-+}}{11}\right]\right)\right]}{\mu N / 2} \\
& =\frac{2 P\left(\begin{array}{c}
- \\
1
\end{array}\right)}{\mu^{2} N}\left\{\mu \left[\left\langle\mathcal{N}\left[\begin{array}{c}
\overline{\overline{1}}+\overline{0} \\
10
\end{array}\right]+\langle\mathcal{N}[\overline{\overline{-+}}]\rangle+p_{o}\left(\left\langle\mathcal{N}\left[\frac{\overline{--}}{10}\right]\right\rangle+\left\langle\mathcal{N}\left[\frac{\overline{-+}}{11}\right]\right\rangle\right)\right]\right.\right.
\end{aligned}
$$

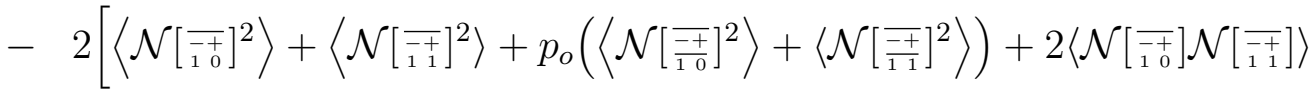

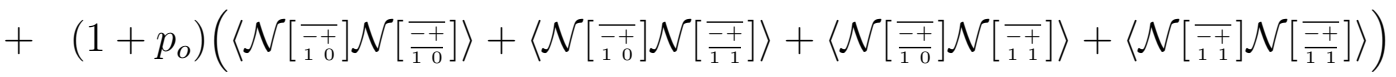

$$
\begin{aligned}
& \left.\left.\left.+2 p_{o}\left\langle\mathcal{N}\left[\frac{-\overline{10}}{10}\right] \mathcal{N}\left[\frac{-+}{11}\right]\right\rangle\right)\right]\right\} \\
& =\frac{2 P\left(\begin{array}{c}
- \\
1
\end{array}\right)}{\mu N}\left\{(\mu-2)\left[P\left[\begin{array}{c}
\overline{-+} \\
10
\end{array}\right]+P\left[\begin{array}{c}
\overline{-+} \\
11
\end{array}\right]+p_{o}\left(P\left[\frac{\overline{\frac{-+}{10}}}{10}\right]+P\left[\frac{\overline{-+}}{11}\right]\right)\right]\right. \\
& -2(\mu-1)\left[\left(P\left[\begin{array}{c}
\overline{-+} \\
10
\end{array}\right]+P\left[\begin{array}{c}
\overline{-+} \\
11
\end{array}\right]\right)^{2}+p_{o}\left(P\left[\frac{\overline{\frac{-t}{10}}}{10}\right]+P\left[\frac{\overline{-+}}{11}\right]\right)^{2}+\right.
\end{aligned}
$$

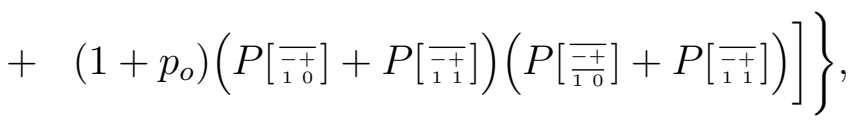

donde hemos usado los momentos de las Ecs. (D.7). Después de sustituir expresiones C.10 por las probabilidades $P\left[\begin{array}{l}\overline{-+} \\ 1 \mathcal{D}\end{array}\right]$ y $P\left[\frac{\overline{-+}}{\overline{1 D}}\right]$ llegamos a

$$
\begin{array}{r}
\left.\Delta \rho^{+-}\right|_{-\rightarrow_{1}^{+}}=\frac{\rho^{+-}}{\mu N}\left\{(\mu-2)\left[(1-q) \rho_{1}+q p_{o}\left(\rho_{11}+\frac{\rho_{10}}{2}\right)\right]\right. \\
\left.-(\mu-1) \frac{\rho^{+-} \rho_{1}}{\rho^{-}}\left[1-\left(1-p_{o}\right) q\right]\right\} .
\end{array}
$$

Sumando las Ecs. (D.9) y (D.11) podemos obtener la siguiente expresión para el cambio en $\rho^{+-}$debido a las transiciones $-\rightarrow+$

$$
\left.\frac{d \rho^{+-}}{d t}\right|_{-\rightarrow+}=\frac{\omega \rho^{+-}}{\mu}\left[\mu-2-(\mu-1) \frac{\rho^{+-}}{\rho^{-}}\right] .
$$

Luego, intercambiando los signos $+\mathrm{y}-$ en la Ec. (D.12) obtenemos el cambio en $\rho^{+}$debido a las transiciones $+\rightarrow-$

$$
\left.\frac{d \rho^{+-}}{d t}\right|_{+\rightarrow-}=\frac{\omega \rho^{+-}}{\mu}\left[\mu-2-(\mu-1) \frac{\rho^{+-}}{\rho^{+}}\right] .
$$

Finalmente, sumando las Ecs. (D.12) y (D.13) llegamos a la siguiente ecuación de evolución para $\rho^{+-}$mencionada en la Ec. 3.3b del capítulo 3 de esta tesis.

$$
\frac{d \rho^{+-}}{d t}=\frac{2 \omega \rho^{+-}}{\mu}\left[(\mu-1)\left(1-\frac{\rho^{+-}}{2 \rho^{+} \rho^{-}}\right)-1\right] \text {. }
$$





\section{Apéndice E}

\section{Deducción de la ecuación de evolución para $\rho_{1}$}

El cambio promedio de la fracción de nodos infectados $\rho_{1}$ en un solo paso de tiempo se puede escribir como

$$
\frac{d \rho_{1}}{d t}=\frac{1}{1 / N}\left[\left.\Delta \rho_{1}\right|_{+_{1}^{+} \rightarrow_{0}^{+}}+\left.\Delta \rho_{1}\right|_{+_{0}^{+} \rightarrow_{1}^{+}}+\left.\Delta \rho_{1}\right|_{-\rightarrow_{0}^{-}}+\left.\Delta \rho_{1}\right|_{-_{0} \rightarrow_{1}^{-}}\right]
$$

donde cada término representa una transición diferente que corresponde a una actualización de enfermedad en la capa de contacto. El primer término de la Ec. (E.1) corresponde a la recuperación de un nodo $\left[\begin{array}{l}+ \\ 1\end{array}\right]$, y puede ser estimado como

$$
\left.\Delta \rho_{1}\right|_{+_{1} \rightarrow_{0}^{+}}=-P\left(\begin{array}{c}
+ \\
1
\end{array}\right)(1-\beta) \frac{1}{N} \simeq-\frac{(1-\beta)}{N} \rho^{+} \rho_{1} .
$$

Esto es, con probabilidad $P\left(\begin{array}{c}+ \\ 1\end{array}\right) \simeq \rho^{+} \rho_{1}$ un nodo $\left[\begin{array}{c}+ \\ 1\end{array}\right]$ es elegido al azar, y luego se recupera con probabilidad $1-\beta$, disminuyendo $\rho_{1}$ en $1 / N$. El segundo término corresponde a la infección de un nodo $\left[\begin{array}{l}+ \\ 0\end{array}\right]$, mientras que los dos últimos términos son equivalentes a los dos primeros, pero donde se recupera un nodo con opinión -, respectivamente. Por la simetría de las opiniones $+\mathrm{y}-$, los últimos dos términos se obtienen intercambiando los signos $+\mathrm{y}-$ en los dos primeros.

Ahora encontramos una expresión aproximada para el segundo término de la Ec. E.1. Un nodo susceptible $j$ con estado $\left[\begin{array}{l}+ \\ 0\end{array}\right]$ puede ser infectado por un vecino enfermo $i$ con opinión + o - que esté conectado a $j$ por un link de contacto o por ambos tipos de links. Por lo tanto, las cuatro posibles interacciones de contacto $\left[\begin{array}{l}+ \\ 0\end{array}\right] \rightarrow\left[\begin{array}{l}+ \\ 1\end{array}\right]$ conducen a la transición:

$$
\left.\Delta \rho_{1}\right|_{+_{0} \rightarrow_{1}^{+}}=\left.\Delta \rho_{1}\right|_{\frac{++}{10} \rightarrow \frac{++}{11}}+\left.\Delta \rho_{1}\right|_{\frac{\overline{ }}{10} \rightarrow \frac{\overline{ }}{11}}+\left.\Delta \rho_{1}\right|_{\frac{-+}{10} \rightarrow \frac{-+}{11}}+\left.\Delta \rho_{1}\right|_{\frac{\overline{\frac{+}{10}} \rightarrow \frac{\overline{ }}{11}}{10}} .
$$


El símbolo $\left[\begin{array}{l}\overline{\mathcal{O}}+ \\ 10\end{array}\right]$ representa una interacción de contacto entre el nodo $i$ con estado $\left[\begin{array}{l}\mathcal{O} \\ 1\end{array}\right](\mathcal{O}=+,-)$ y el nodo $j$ con estado $\left[\begin{array}{l}+ \\ 0\end{array}\right]$. El estado que cambia en la interacción ahora se muestra en el lado derecho del símbolo, mientras que el lado izquierdo se utiliza para el caso de las interacciones sociales descritas en las secciones anteriores. Esto se debe a que el vecino elegido $j$ de $i$ cambia de estado en el $\mathrm{CP}$, mientras que en el VM es el nodo $i$ quien cambia de estado. Teniendo en cuenta los eventos y sus probabilidades asociadas que conducen a cada una de las cuatro interacciones descritas anteriormente, podemos escribir la Ec. (E.3) como

$$
\begin{aligned}
\left.\Delta \rho_{1}\right|_{0} ^{+\rightarrow_{1}^{+}} & =P\left(\begin{array}{c}
+ \\
1
\end{array}\right) \sum_{\left\{\mathcal{N}_{1}^{+}\right\}}^{\mu} M\left(\left\{\mathcal{N}_{1}^{+}\right\}, \mu\right) \frac{\beta}{\mu}\left(\mathcal{N}\left[\frac{++}{10}\right]+\mathcal{N}\left[\frac{++}{10}\right]\right) \frac{1}{N} \\
& +P\left(\begin{array}{c}
- \\
1
\end{array}\right) \sum_{\left\{\mathcal{N}_{1}^{-}\right\}}^{\mu} M\left(\left\{\mathcal{N}_{1}^{-}\right\}, \mu\right) \frac{\beta}{\mu}\left(\mathcal{N}\left[\frac{-+}{10}\right]+p_{d} \mathcal{N}\left[\frac{-+}{10}\right]\right) \frac{1}{N} .
\end{aligned}
$$

El primer y el tercer término de la Ec. (E.4) corresponden a seleccionar un nodo $i$ con estado $\left[\begin{array}{l}\mathcal{O} \\ 1\end{array}\right]$ y un vecino de contacto $j$ al azar con estado $\left[\begin{array}{l}+ \\ 0\end{array}\right]$, lo cual ocurre con probabilidad $P\left(\begin{array}{c}\mathcal{O} \\ 1\end{array}\right) \mathcal{N}\left[\frac{\mathcal{O}+}{10}\right] / \mu$, y luego $i$ infecta a $j$ con probabilidad $\beta$, dado que no están conectados por un enlace social. El segundo y cuarto término son similares al primer y segundo término, respectivamente, pero seleccionando un vecino social/de contacto $j$. Como ambos tipos de enlaces están presentes en este caso, $i$ infecta a $j$ con probabilidad $\beta p_{d}$ cuando ambos nodos tienen opiniones diferentes (cuarto término). En todos los casos $\rho_{1}$ cambia en $1 / N$. Realizando las sumatorias de la Ec. (E.4) obtenemos

$$
\left.\Delta \rho_{1}\right|_{0} ^{+\rightarrow_{1}^{+}}=\frac{\beta}{\mu N}\left[P\left(\begin{array}{c}
+ \\
1
\end{array}\right)\left(\left\langle\mathcal{N}\left[\frac{++}{10}\right]\right\rangle+\left\langle\mathcal{N}\left[\frac{\mp+}{10}\right]\right\rangle\right)+P\left(\begin{array}{c}
- \\
1
\end{array}\right)\left(\left\langle\mathcal{N}\left[\frac{-+}{10}\right]\right\rangle+p_{d}\left\langle\mathcal{N}\left[\frac{\overline{-+}}{10}\right]\right\rangle\right)\right](\mathrm{E} .5)
$$

Reemplazando las expresiones por los primeros momentos $\left\langle\mathcal{N}\left[\frac{\mathcal{O}+}{10}\right]\right\rangle=\mu P\left[\frac{\mathcal{O}+}{10}\right]$ y $\left\langle\mathcal{N}\left[\overline{\frac{\mathcal{O}}{10}}\right]\right\rangle=\mu P\left[\overline{\frac{\mathcal{O}}{10}}\right]$ in Ec. (E.5), y usando las siguientes expresiones para las probabilidades condicionales

$$
\begin{aligned}
& P\left[\frac{++}{10}\right] \simeq \frac{(1-q) \rho^{+} \rho_{10}}{2 \rho_{1}}, \quad P\left[\overline{\frac{++}{10}}\right] \simeq \frac{q \rho^{++} \rho_{10}}{2 \rho^{+} \rho_{1}} \\
& P\left[\frac{-+}{10}\right] \simeq \frac{(1-q) \rho^{+} \rho_{10}}{2 \rho_{1}}, \quad P\left[\frac{-+}{\frac{-+}{10}}\right] \simeq \frac{q \rho^{+-} \rho_{10}}{4 \rho^{-} \rho_{1}}
\end{aligned}
$$

finalmente llegamos a

$$
\left.\Delta \rho_{1}\right|_{+_{0}^{+} \rightarrow_{1}^{+}} \simeq \frac{\beta \rho_{10}}{2 N}\left[\rho^{+}-\frac{q}{2}\left(1-p_{d}\right) \rho^{+-}\right]
$$

donde hemos usado las relaciones de conservación de las Ecs. 3.1a y 3.1c). 
Ahora que estimamos los primeros dos términos de la Ec. (E.1), los dos últimos términos son obtenidos intercambiando los signos $+\mathrm{y}-$ en las Ecs. (E.2) y (E.8):

$$
\begin{aligned}
& \left.\Delta \rho_{1}\right|_{{ }_{-} \rightarrow_{0}^{-}} \simeq-\frac{(1-\beta)}{N} \rho^{-} \rho_{1}, \\
& \left.\Delta \rho_{1}\right|_{{ }_{0} \rightarrow_{1}^{-}} \simeq \frac{\beta \rho_{10}}{2 N}\left[\rho^{-}-\frac{q}{2}\left(1-p_{d}\right) \rho^{+-}\right] .
\end{aligned}
$$

Sumando las Ecs. (E.2), (E.8), E.9) y (E.10), la ecuación de evolución para $\rho_{1}$ (E.1) se convierte en

$$
\frac{d \rho_{1}}{d t} \simeq \frac{\gamma \beta \rho_{10}}{2}-(1-\beta) \rho_{1}
$$

con

$$
\gamma \equiv 1-q\left(1-p_{d}\right) \rho^{+-},
$$

como se menciona en las Ecs. (3.5a) y (3.6) del capítulo 3 de esta tesis. 



\section{Apéndice F}

\section{Deducción de la ecuación de evolución para $\rho_{10}$}

El cambio promedio de la fracción de pares de nodos susceptibles infectados $\rho_{10}$ en un solo paso de tiempo se puede escribir como

$$
\frac{d \rho_{10}}{d t}=\frac{1}{1 / N}\left[\left.\Delta \rho_{10}\right|_{1_{1} \rightarrow_{0}^{+}}+\left.\Delta \rho_{10}\right|_{0_{+} \rightarrow_{1}^{+}}\right]+\frac{1}{1 / N}\left[\left.\Delta \rho_{10}\right|_{+_{1}^{+} \rightarrow_{0}^{+}}+\left.\Delta \rho_{10}\right|_{0_{0} \rightarrow_{1}^{+}}\right]^{+\Leftrightarrow-}
$$

donde el primer y segundo término corresponden al cambio en $\rho_{10}$ debido a la recuperación de un nodo $\left[\begin{array}{l}+ \\ 1\end{array}\right]$ y a la infección de otro nodo $\left[\begin{array}{l}+ \\ 0\end{array}\right]$, respectivamente, mientras que los dos últimos términos son los eventos de recuperación e infección correspondientes de los nodos con opinión -, y se obtienen intercambiando los signos $+\mathrm{y}-$ en los primeros dos términos. El término de recuperación se puede calcular como

$$
\begin{aligned}
\left.\Delta \rho_{10}\right|_{1_{1} \rightarrow_{0}^{+}} & =P\left(\begin{array}{c}
+ \\
1
\end{array}\right)(1-\beta) \times \\
& \times \sum_{\left\{\mathcal{N}_{1}^{+}\right\}}^{\mu} M\left(\left\{\mathcal{N}_{1}^{+}\right\}, \mu\right) \frac{\left[\mu-2\left(\mathcal{N}\left[\frac{++}{10}\right]+\mathcal{N}\left[\frac{++}{10}\right]+\mathcal{N}\left[\frac{+-}{10}\right]+\mathcal{N}\left[\frac{+-}{10}\right]\right)\right]}{\mu N / 2},
\end{aligned}
$$

donde la expresión entre corchetes es el cambio en el número de links 10 conectados a un nodo $i$ con estado $\left[\begin{array}{c}+ \\ 1\end{array}\right]$ cuando $i$ se recupera, dada una configuración específica de tipos de nodos conectados a $i$ [ver Fig. F.1(a)]. La sumatoria en la Ec. (F.2) conduce a los primeros momentos de la probabilidad multinomial $M\left(\left\{\mathcal{N}_{1}^{+}\right\}, \mu\right)$, con probabilidades de evento único $P\left[\frac{++}{10}\right]$ y $P\left[\frac{\mp+}{10}\right]$ dadas por las Ecs. (E.6), y

$$
P\left[\frac{+-}{10}\right] \simeq \frac{(1-q) \rho^{-} \rho_{10}}{2 \rho_{1}}, \quad P\left[\frac{+-}{10}\right] \simeq \frac{q \rho^{+-} \rho_{10}}{4 \rho^{+} \rho_{1}}
$$



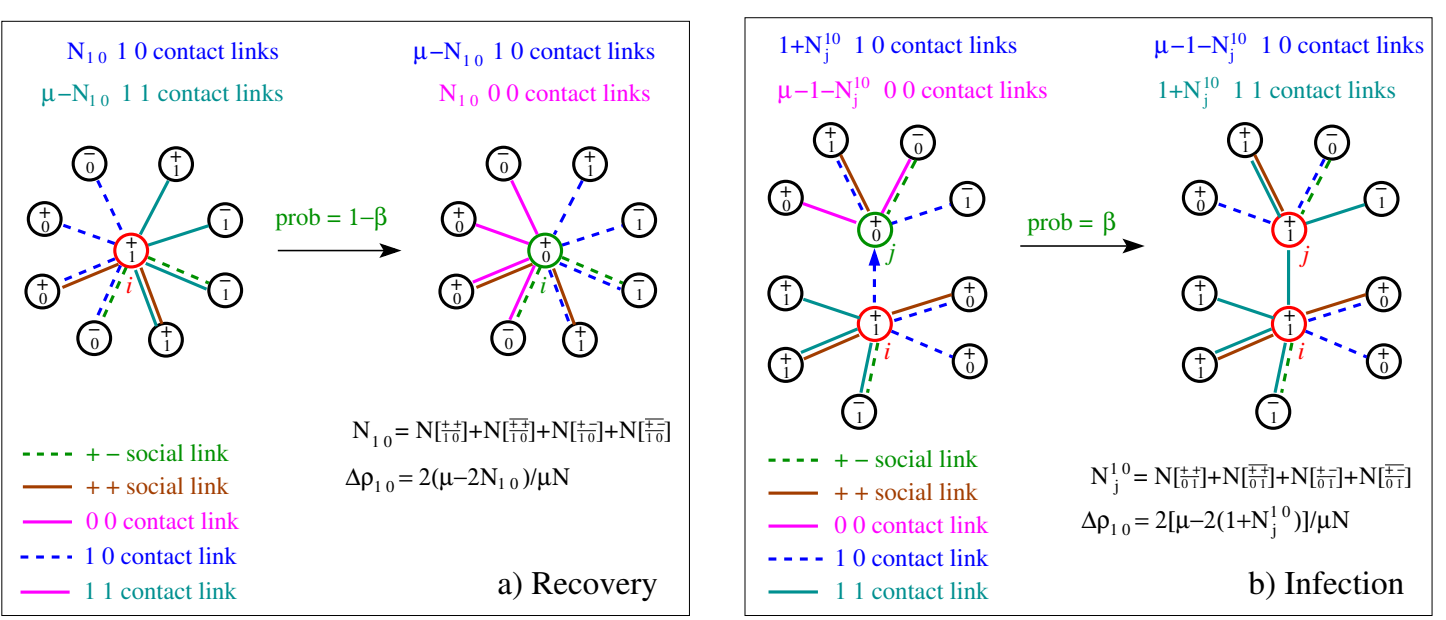

Figura F.1: (Color online) Ilustración esquemática de dos actualizaciones de enfermedad. (a) Recuperación: un nodo $i$ con estado $\left[\begin{array}{l}+ \\ 1\end{array}\right]$ se recupera con probabilidad $1-\beta$. (b) Infección: un nodo $i$ con estado $\left[\begin{array}{l}+ \\ 1\end{array}\right]$ infecta a un vecino de contacto $j$ en estado $\left[\begin{array}{c}+ \\ 0\end{array}\right]$ con probabilidad $\beta$. El cambio en la densidad de links de contacto 10 es denotado por $\Delta \rho_{10}$.

Reemplazando estas expresiones por las probabilidades y usando las relaciones de conservación de las Ecs. (3.1a) y 3.1c) obtenemos, después de hacer un poco de álgebra,

$$
\left.\Delta \rho_{10}\right|_{1_{1} \rightarrow_{0}^{+}} \simeq \frac{2(1-\beta) \rho^{+}}{N}\left(\rho_{1}-\rho_{10}\right)
$$

Ahora calcularemos el segundo término de la Ec. (F.1) correspondiente al cambio en $\rho_{10}$ después de la infección de un nodo con opinión + :

$$
\begin{aligned}
& \left.\Delta \rho_{10}\right|_{+_{0} \rightarrow_{1}^{+}}=\left[P\left(\begin{array}{c}
+ \\
1
\end{array}\right) \sum_{\left\{\mathcal{N}_{i, 1}^{+}\right\}}^{\mu} M\left(\left\{\mathcal{N}_{i, 1}^{+}\right\}, \mu\right) \frac{\beta}{\mu}\left(\mathcal{N}_{i}\left[\frac{++}{10}\right]+\mathcal{N}_{i}\left[\frac{\mp+}{10}\right]\right)\right. \\
& \left.+P\left(\frac{-}{1}\right) \sum_{\left\{\mathcal{N}_{i, 1}^{-}\right\}}^{\mu} M\left(\left\{\mathcal{N}_{i, 1}^{-}\right\}, \mu\right) \frac{\beta}{\mu}\left(\mathcal{N}_{i}\left[\frac{-+}{10}\right]+p_{d} \mathcal{N}_{i}\left[\frac{\overline{-}}{10}\right]\right)\right] \\
& \times \sum_{\left\{\mathcal{N}_{j, 0}^{+}\right\}}^{\mu-1} M\left(\left\{\mathcal{N}_{j, 0}^{+}\right\}, \mu-1\right) \frac{\left[\mu-2\left(1+\mathcal{N}_{j}\left[\frac{++}{01}\right]+\mathcal{N}_{j}\left[\overline{\frac{++}{01}}\right]+\mathcal{N}_{j}\left[\frac{+-}{01}\right]+\mathcal{N}_{j}\left[\overline{\frac{+-}{01}}\right]\right)\right]}{\mu N / 2} \\
& =\mathcal{P} \times \mathcal{C} \text {. }
\end{aligned}
$$

El término llamado $\mathcal{P}$ en la Ec. (F.5) -las dos sumas dentro de los corchetes- es la probabilidad de que un nodo $i$ con estado $\left[\begin{array}{l}\mathcal{O} \\ 1\end{array}\right]$ infecte a un vecino $j$ con estado $\left[\begin{array}{c}+ \\ 0\end{array}\right]$, y es la misma calculada en la Ec. E.4 para $\left.\Delta \rho_{1}\right|_{0_{+}^{+} \rightarrow_{1}^{+}}$, que se estima en la 
Ec. E.8) como

$$
\mathcal{P} \simeq \frac{\beta \rho_{10}}{2}\left[\rho^{+}-\frac{q}{2}\left(1-p_{d}\right) \rho^{+-}\right] .
$$

Notamos que el prefactor extra $1 / N$ en la Ec. (E.8) proviene del cambio en $\rho_{1}$, que para $\rho_{10}$ depende de los vecinos del nodo $j$. El subíndice $i$ en el término $\mathcal{P}$ indica que el término de probabilidad de infección depende solo del nodo $i$ y sus vecinos [ver Fig. F.1(b)]. El término llamado $\mathcal{C}$ correspondiente a la suma fuera de los corchetes expresa el cambio en $\rho_{10}$ cuando el nodo $j$ se infecta [ver Fig. F.1(b)]. El subíndice $j$ se refiere al nodo $j$ y sus vecinos. Este término contiene la información de que la infección en $j$ proviene de uno de sus vecinos infectados $i$, y por lo tanto ya se sabe que al menos uno de los vecinos de $j$ tiene estado de enfermedad $\mathcal{D}_{i}=1$. Esto se tiene en cuenta realizando la suma de los otros $\mu-1$ vecinos desconocidos y considerando que el número de enlaces 10 conectados a $j$ es al menos uno, que se suma al número total de enlaces 10 dentro de los paréntesis. Usando las probabilidades condicionales

$$
\begin{array}{ll}
P\left[\frac{++}{01}\right] \simeq \frac{(1-q) \rho^{+} \rho_{10}}{2 \rho_{0}}, \quad P\left[\frac{\overline{+}}{01}\right] \simeq \frac{q \rho^{++} \rho_{10}}{2 \rho^{+} \rho_{0}} \\
P\left[\frac{+-}{01}\right] \simeq \frac{(1-q) \rho^{-} \rho_{10}}{2 \rho_{0}}, & P\left[\frac{+-}{01}\right] \simeq \frac{q \rho^{+-} \rho_{10}}{4 \rho^{+} \rho_{0}},
\end{array}
$$

y las relaciones de conservación de las Ecs. (3.1b) y (3.1c), el término de cambio se convierte

$$
\mathcal{C} \simeq \frac{2}{\mu N}\left[(\mu-1)\left(1-\frac{\rho_{10}}{\rho_{0}}\right)-1\right] .
$$

Finalmente, combinando las Ecs. $(\overline{F .6})$ y $($ F.9 para $\mathcal{P}$ y $\mathcal{C}$ llegamos a

$$
\left.\Delta \rho_{10}\right|_{0} ^{+} \rightarrow_{1}^{+} \simeq \frac{\beta \rho_{10}}{\mu N}\left[\rho^{+}-\frac{q}{2}\left(1-p_{d}\right) \rho^{+-}\right]\left[(\mu-1)\left(1-\frac{\rho_{10}}{\rho_{0}}\right)-1\right] .
$$

Luego, sumando los términos de recuperación e infección de las Ecs. (F.4) y (F.10), respectivamente, obtenemos los primeros dos términos de la Ec. (F.1), mientras que los últimos dos términos son obtendios intercambiando los signos $+\mathrm{y}-$ en la última expresión. Sumando estos cuatro términos llegamos a lal siguiente ecuación de evolución para $\rho_{10}$

$$
\frac{d \rho_{10}}{d t} \simeq \frac{\gamma \beta \rho_{10}}{\mu}\left[(\mu-1)\left(1-\frac{\rho_{10}}{\rho_{0}}\right)-1\right]+2(1-\beta)\left(\rho_{1}-\rho_{10}\right),
$$

mencionada en la Ec. (3.5b) del capítulo 3 de esta tesis. 



\section{Bibliografía}

[1] C. Castellano, S. Fortunato, and V. Loreto. Statistical physics of social dynamics. Rev. Mod. Phys., 81(2):591, 2009.

[2] B. Latané. The psychology of social impact. American Psychologist, 36:343356, 1981.

[3] B. Latané and A. Nowak. Progress in Communication Science: Persuasion, Vol 13. Norwood, NJ: Ablex), 1997.

[4] Peter Clifford and Aidan Sudbury. A model for spatial conflict. Biometrika, 60(3):581-588, January 1973.

[5] R. Holley and T. M. Liggett. Ergodic theorems for weakly interacting infinite systems and the voter model. Ann. Probab., 3:643, 1975.

[6] Federico Vazquez and Víctor M. Eguíluz. Analytical solution of the voter model on uncorrelated networks. New Journal of Physics, 10(6):063011, 2008.

[7] M Ángeles Serrano, Konstantin Klemm, Federico Vazquez, Víctor M Eguíluz, and Maxi San Miguel. Conservation laws for voter-like models on random directed networks. Journal of Statistical Mechanics: Theory and Experiment, 2009(10):P10024, 2009.

[8] S Galam. Minority opinion spreading in random geometry. Eur. Phys. J. B, 25:403-406, 2002.

[9] P.L Krapivsky and S. Redner. Dynamics of majority rule in two-state interacting spin systems. Phys. Rev. L., 90:238701, 2003.

[10] W. O. Kermack and A. G. McKendrick. Proc. Roy. Soc. Lond. A, 115:700$721,1927$. 
[11] N. T. J. Bailey. The Mathematical Theory of Epidemics. Griffin, London, 1957.

[12] R. M. Anderson and R. M. May. Infectious Diseases of Humans: Dynamics and Control. Oxford University Press, Oxford, 1991.

[13] K. Maciej and T. Patrick. Layered complex networks. Phys. Rev. L., 96:138701, 2003.

[14] M. Szell, R. Lambiotte, and S. Thurner. Multirelational organization of largescale social networks in an online world. PNAS, 107:13636-13641, 2010.

[15] P. J. Mucha, T. Richardson, K. Macon, M. A. Porter, and J-P. Onnela. Community Structure in Time-Dependent, Multiscale, and Multiplex Networks. Science, 328:876, 2010.

[16] A. Saumell-Mendiola, M. Ángeles Serrano, and M. Boguná. Epidemic spreading on interconnected networks. Physical Review E, 86:026106, 2012.

[17] S. Gómez, A. Díaz-Guilera, J. Gómez-Gardeñes, C. J. Pérez-Vicente, Y. Moreno, and A. Arenas. Diffusion Dynamics on Multiplex Networks. Phys. Rev. Lett., 110:028701, 2013.

[18] L Onsager. Crystal statistics. i. a two-dimensional model with an order disorder transition. Physical Review, 65:117-149, 1944.

[19] D. J. Watts and S. H. Strogatz. Nature, 393:440-442, 1998.

[20] A.-L. Barabási and R. Albert. Emergence of scaling in random networks. Science, 286:509-512, 1999.

[21] P. Erdös and A. y Rényi. On random graphs i. Publicationes Mathematicae (Debrecen), 6:290-297, 1959.

[22] S. Boccaletti, G. Bianconi, R. Criado, C. I. Del Genio, J. Gómez-Gardeñes, M. Romance, I. Sendiña-Nadal, Z. Wang, and M. Zanin. The structure and dynamics of multilayer networks. physics reports. Physics Reports, 544(1):1$122,2014$.

[23] Serge Galam. Plastic crystals, melting, and random fields. Physics Letters A, 122(5):271-274, 1987. 
[24] S Galam and M Gabay. Coupled spin systems and plastic crystals. EPL (Europhysics Letters), 8(2):167, 1989.

[25] S. Redner. A Guide to First-Passage Processes. Cambridge University Press, 2001.

[26] C. E. La Rocca, L. A. Braunstein, and F. Vazquez. The influence of persuasion in opinion formation and polarization. Europhys. Lett., 106(4):40004, 2014.

[27] N. Metropolis, A.W. Rosenbluth, M Rosenbluth, A.H. Teller, and E. Teller. J. Chem. Phys, 21:1087, 1953.

[28] M Plischke and B. Bergersen. Equilibrium Statistical Physics. World Scientific Publishing, 1994.

[29] J. Marro and R. Dickman. Non-equilibrium Phase Transitions in Lattice Models. Cambridge University Press, 1999.

[30] J. Cardy. Scaling and Renormalization is Statistical Physics. Cambridge University Press, 1996.

[31] R. Holley and T. M. Liggett. Ann. Probab., 4:195, 1975.

[32] F. Vazquez, V. M. Eguíluz, and M. S. Miguel. Phys. Rev. Lett., 100:108702, 2008.

[33] M. E. J. Newman and G.T Barkema. Monte Carlo Methods in Statistical Physics. Oxford University Press., 1999.

[34] D.T Gillespie. Exact stochastic simulation of coupled chemical reagctions. J. Phys. Chem, 81:2340-2361, 1977.

[35] Myers D. G. Group Decision Making. Academic Press, New York, London, 1982.

[36] Isenberg D. J. Group polarization: A critical review and meta-analysis. Journal of personality and social psychology, 50:1141, 1986.

[37] Lau D. C. and Murnighan J. K. Demographic diversity and faultlines: The compositional dynamics of organizational groups. Acad. Manag. Rev., 23:325, 1998. 
[38] Michael Mäs and Andreas Flache. Differentiation without distancing. explaining bi-polarization of opinions without negative influence. PLoS ONE, 8(11):1-17, 112013.

[39] Michael Mäs, Andreas Flache, Károly Takács, and Karen A Jehn. In the short term we divide, in the long term we unite: Demographic crisscrossing and the effects of faultlines on subgroup polarization. Organization science, 24(3):716-736, 2013.

[40] Weisbuch G, Deffuant G, Amblard F., and Nadal J.-P. Meet, discuss, and segregate! Complexity, 7:55, 2002.

[41] E. Ben-Naim, P.L Krapivsky, F. Vazquez, and S. Redner. Unity and discord in opinion dynamics. Physica A, 330:99106, 2003.

[42] E. Ben-Naim, P. L. Krapivsky, and S. Redner. Physica D, 83:190204, 2003.

[43] D. Bernoulli. Histoire de l'Acad. Roy. Sci. (Paris) avec M. des Math. et Phys. 1760 .

[44] J. P. Sturmberg and C. M. Martin. Handbook of Systems and Complexity in Health. Springer, 2013.

[45] M. E. J. Newman. Networks: An Introduction. Oxford University Press, 2010.

[46] A. Mata, R. Ferreira, and S. Ferreira. Heterogeneous pair-approximation for the contact process on complex networks. New Journal of Physics, 16:053006, 2014.

[47] Emanuele Massaro and Franco Bagnoli. Epidemic spreading and risk perception in multiplex networks: A self-organized percolation method. Physical Review E, 90(5):052817, 2014.

[48] Clara Granell, Sergio Gómez, and Alex Arenas. Dynamical interplay between awareness and epidemic spreading in multiplex networks. Phys. Rev. Lett., 111:128701, 2013.

[49] Clara Granell, Sergio Gómez, and Alex Arenas. Competing spreading processes on multiplex networks: awareness and epidemics. Physical Review E, 90(1):012808, 2014. 
[50] Wei Wang, Ming Tang, Hui Yang, Younghae Do, Ying-Cheng Lai, and GyuWon Lee. Asymmetrically interacting spreading dynamics on complex layered networks. Scientific Reports, 4:5097, 2014.

[51] R.P. Abelson. Mathematical Models of the Distribution of Attitudes Under Controversy in Contributions to Mathematical Psychology. New York: Rinehart Winston, 1964.

[52] P. Bonacich and P. Lu. Introduction to Mathematical Sociology. Princeton and Oxford: Princeton University Press, 2012.

[53] N.P. Mark. Culture and competition: Homophily and distancing explanations for cultural niches. Am. Sociol Rev, 68:319-345, 2003.

[54] A. Flache and M.W. Macy. Small worlds and cultural polarization. J. Math Sociol, 35:146-176, 2011.

[55] T. Vaz Martins, M. Pineda, and R. Toral. Mass media and repulsive interactions in continuous-opinion dynamics. EPL, 91:48003, 2010.

[56] M. McPherson, L. Smith-Lovin, and J. M. Cook. Birds of a feather: Homophily in social networks. Annual Review of Sociology, 27:415-444, 2001.

[57] H. Ibarra. Homophily and differential returns-sex-differences in network structure and access in an advertising firm. Administrative Science Quarterly, 37:422-447, 1992.

[58] A. Vinokur and E. Burnstein. Depolarization of attitudes in groups. Journal of Personality and Social Psychology, 36:872-885, 1978.

[59] N Crokidakis and C. Anteneodo. Role of conviction in nonequilibrium models of opinion formation. Phys. Rev. E, 86:061127, 2012.

[60] N. Crokidakis. Role of noise and agents' convictions on opinion spreading in a three-state voter-like model. J. Stat. Mech., 2013:P07008, 2013.

[61] P. Balenzuela, J.P Pinasco, and V. Semeshenko. The undecided have the key: interaction-driven opinion dynamics in a three state model. Plos One, 10:e0139572, 2015.

[62] G. R. Terranova, J. A. Revelli, and G. J. Sibona. Active speed role in opinion formation of interacting moving agents. EPL, 105:30007, 2014. 
[63] M. Pineda, R. Toral, and E. Hernández-García. Noisy continuous-opinion dynamics. Journal of Statistical Mechanics: Theory and Experiment, 2009:P08001, 2009.

[64] X. Castelló, V.M Eguíluz, and M. SanMiguel. Ordering dynamics with two non-excluding options: bilingualism in language competition. New Journal of Physics, 8:308, 2006.

[65] D. Volovik and S. Redner. Dynamics of confident voting. Journal of Statistical Mechanics: Theory and Experiment, page P04003, 2012.

[66] L Dall'Asta and T. Galla. Algebraic coarsening in voter models with intermediate states. J. Phys. A: Math. Theor., 41:435003, 2008.

[67] F Vazquez and C. López. Systems with two symmetric absorbing states: relating the microscopic dynamics with the macroscopic behavior. Physical Review E, 78:061127, 2008.

[68] F. Vazquez, X. Castelló, and M. San Miguel. Agent based models of language competition: macroscopic descriptions and order-disorder transitions. Journal of Statistical Mechanics: Theory and Experiment, 2010(04):P04007, 2010.

[69] I. Dornic, H. Chaté, J. Chave, and H. Hinrichsen. Critical coarsening without surface tension: The universality class of the voter model. Phys. Rev. Lett., 87:045701, 2001.

[70] J.D. Gunton, M. San Miguel, and P. S. Sahni. Phase Transitions and Critical Phenomena. London Academic Press, 1983.

[71] A.J. Bray. Theory of phase-ordering kinetics. Adv. Phys., 51:481, 2003.

[72] P. Chen and S. Redner. Majority rule dynamics in finite dimensions. Phys. Rev. E, 71:036101, 2005.

[73] L.F. Cugliandolo. Critical percolation in bidimensional coarsening. Journal of Statistical Mechanics: Theory and Experiment, 11:114001, 2016.

[74] M. Plischke, Z. Rácz, and D. Liu. Time-reversal invariance and universality of two-dimensional growth models. Phys. Rev. B., 35:3485, 1987. 
[75] V. Spirin, P.L Krapivsky, and S. Redner. Fate of zero-temperature ising ferromagnets. Phys. Rev. E., 63:036118, 2001.

[76] T.C. Schelling. Dynamic models of segregation. J Math Sociol, 1:143-186, 1971.

[77] A. Barrat, M. Barthélemy, and A. Vespignani. Dynamical Processes on Complex Networks. Cambridge University Press, 2008.

[78] Romualdo Pastor-Satorras, Claudio Castellano, Piet Van Mieghem, and Alessandro Vespignani. Epidemic processes in complex networks. Rev. Mod. Phys., 87:925-979, Aug 2015.

[79] Thomas M. Liggett. Interacting Particle Systems. Springer, Berlin ; New York, November 2004.

[80] T. E. Harris. Contact interactions on a lattice. Ann. Prob., 2:969-988, 1974.

[81] R. Ferreira and S. Ferreira. Critical behavior of the contact process on smallworld networks. European Physical Journal B, 86:1-7, 2013.

[82] M. De Domenico, A. Sole-Ribalta, E. Cozzo, M. Kivela, Y. Moreno, M. A. Porter, S. Gomez, and A. Arenas. Mathematical formulation of multilayer networks. Physical Review X, 3:041022, 2013.

[83] M. Kivelä, A. Arenas, M. Barthélemy, J. P. Gleeson, Y. Moreno, and M. A. Porter. Multilayer Networks. Journal of Complex Networks, 2:203-271, 2014.

[84] L. G. Alvarez-Zuzek, C. E. La Rocca, F. Vazquez, and L. A. Braunstein. Interacting social processes on interconnected networks. PLOS ONE, 11(9):e0163593, 2016.

[85] A. Halu, K. Zhao, A. Baronchelli, and G. Bianconi. Connect and win: The role of social networks in political elections. Eurphys. Lett., 102:16002, 2013.

[86] Marina Diakonova, Maxi San Miguel, and Victor Eguíluz. Absorbing and shattered fragmentation transitions in multilayer coevolution. Phys. Rev. E, 89:062818, 2014.

[87] M. Diakonova, V. Nicosia, V. Latora, and M. San Miguel. Irreducibility of multilayer network dynamics. New Journal of Physics, 18:023010, 2016. 
[88] F. Vazquez, M. Á. Serrano, and M. San Miguel. Rescue of endemic states in interconnected networks with adaptive coupling. Scientific Reports, 6:29342, 2016.

[89] A. Czaplicka, R. Toral, and M. San Miguel. Competition of simple and complex adoption on interdependent networks. Phys. Rev. E, 94:062301, 2016.

[90] R. Axelrod. The dissemination of culture. Journal of Conflict Resolution, 41(2):203, 1997.

[91] F. Vazquez and S. Redner. Non-monotonicity and divergent time scale in axelrod model dynamics. Eurphys. Lett., 78:18002, 2007.

[92] C. Castellano, D. Vilone, and A. Vespignani. Europhys. Lett., 63:153, 2003.

[93] D. Vilone and C. Castellano. Phys. Rev. E, 69:016109, 2004.

[94] K. Suchecki, V. M. Eguíluz, and M. San Miguel. Europhys. Lett., 69:228, 2005.

[95] C. Castellano, V. Loreto, A. Barrat, F. Cecconi, and D. Parisi. Phys. Rev. E, 71:066107, 2005.

[96] V. Sood and S. Redner. Voter model on heterogeneous graphs. Physical Review Letters, 94(17):178701, May 2005.

[97] C. Castellano, M. Marsili, and A. Vespignani. Phys. Rev. Lett., 85:3536, 2000.

[98] Zhen Wang, Chris T Bauch, Samit Bhattacharyya, Alberto d'Onofrio, Piero Manfredi, Matjaž Perc, Nicola Perra, Marcel Salathé, and Dawei Zhao. Statistical physics of vaccination. Physics Reports, 664:1-113, 2016.

[99] Quarantine-generated phase transition in epidemic spreading.

[100] Piero Manfredi and Alberto D'Onofrio. Modeling the interplay between human behavior and the spread of infectious diseases. Springer Science \& Business Media, 2013.

[101] Sebastian Funk, Erez Gilad, Chris Watkins, and Vincent A A Jansen. The spread of awareness and its impact on epidemic outbreaks. Proceedings of the National Academy of Sciences, 106(16):6872-6877, 2009. 
[102] Quantong Guo, Xin Jiang, Yanjun Lei, Meng Li, Yifang Ma, and Zhiming Zheng. Two-stage effects of awareness cascade on epidemic spreading in multiplex networks. Physical Review E, 91(1):012822, 2015.

[103] Qingchu Wu, Xinchu Fu, Michael Small, and Xin-Jian Xu. The impact of awareness on epidemic spreading in networks. Chaos: An Interdisciplinary Journal of Nonlinear Science, 22(1):013101, 2012.

[104] F. Velásquez-Rojas and F. Vazquez. Opinion dynamics in two dimensions: domain coarsening leads to stable bi-polarization and anomalous scaling exponents. Journal of Statistical Mechanics: Theory and Experiment, 2018:043403, 2018 .

[105] F. Velásquez-Rojas and F. Vazquez. Interacting opinion and disease dynamics in multiplex networks: Discontinuous phase transition and nonmonotonic consensus times. Physical Review E, 95:052315, 2017.

[106] F. Family. Scaling of rough surfaces: effects of surface diffusion. J. Phys. A: Math. Gen, 19:L441-446, 1986.

[107] A.-L. Barabasi and H.E. Stanley. Fractal Concepts in Surface Growth. Cambridge University Press, 1995. 